\title{
Strategic communication
}

\author{
through all stages \\ of a major emergency life cycle, \\ with particular reference to the \\ needs of Ireland
}

Paul Connors

Ph.D. 
Strategic communication through all stages of a major emergency life cycle, with particular reference to the needs of Ireland

Submitted by

Paul Connors B.Comm., M.A.C.C.S.

For the award of Doctor of Philosophy (Ph.D.)

Dublin City University

School of Communications

Supervisor Prof. Colum Kenny

July 2009 


\section{DECLARATION}

I hereby certify that this material, which I now submit for assessment on the programme of study leading to the award of Doctor of Philosophy is entirely my own work, that I have exercised reasonable care to ensure that the work is original, and does not to the best of my knowledge breach any law of copyright, and has not been taken from the work of others save and to the extent that such work has been cited and acknowledged within the text of my work.

\section{Signed:}

ID No.: $\underline{51158302}$

\section{Date:}




\section{TABLE OF CONTENTS}

INTRODUCTION

Overview.

The research question

1.2.1 Conceptual strategic major emergency communication framework .....................................

1.2.2 Best-practice and best-international-practice ............................................................

1.2.3 The approach taken by the Irish government ......................................................5

1.2.4 Link to tactical major emergency communication management .................................5

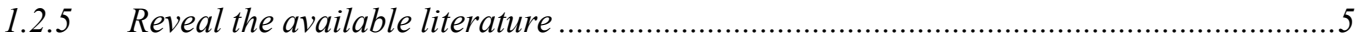

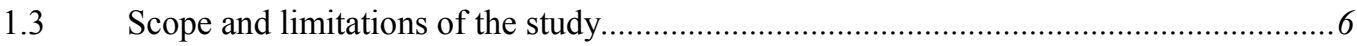

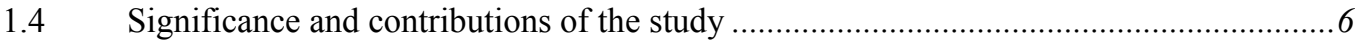

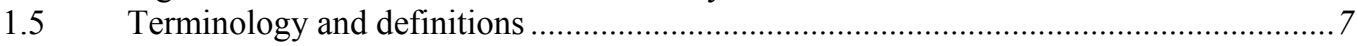

1.5.1 Crisis terminology and definitions ....................................................................... 7

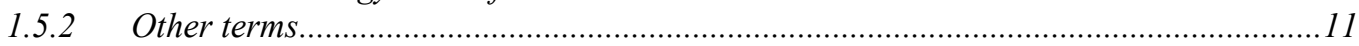

1.5.3 Communication terminology and definitions.................................................... 11

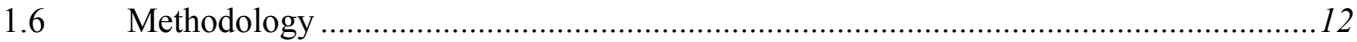

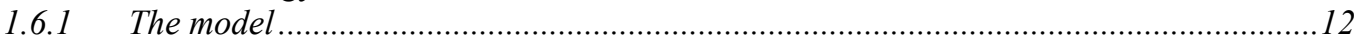

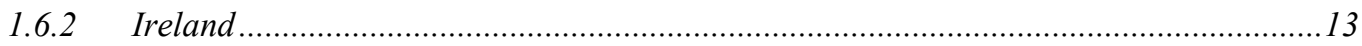

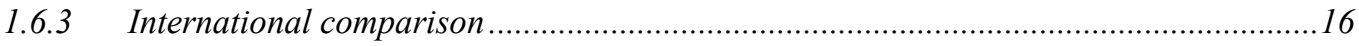

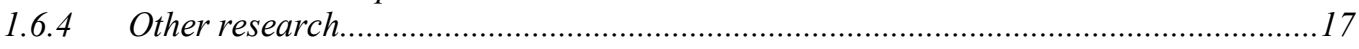

Overview of review of the literature

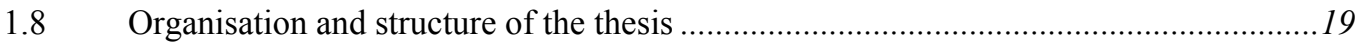

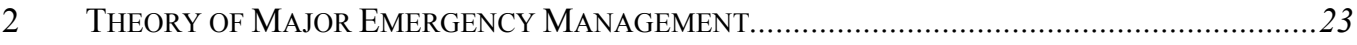

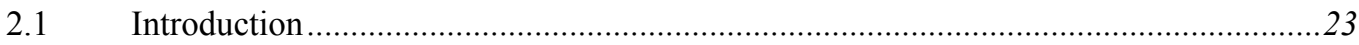

2.2 Overview of major emergencies.........................................................................24

2.2.1 The social construction of major emergencies .........................................................24

2.2.2 Categorisation of major emergencies......................................................................25

2.2.3 Major emergency life cycles ...............................................................................26

$2.3 \quad$ Major emergency management ...........................................................................27

2.3.1 Theory of major emergency management .................................................................2 28

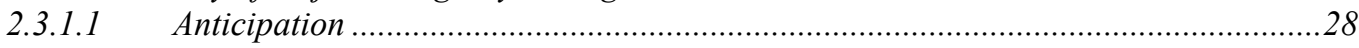

2.3.1.2 Resilience........................................................................................................29

2.3.1.3 Contemporary approaches to major emergency management ..............................29

2.4 The major emergency management cycle ............................................................. 31

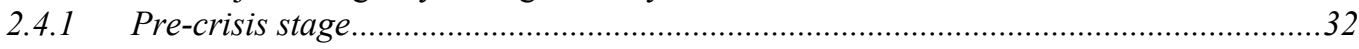

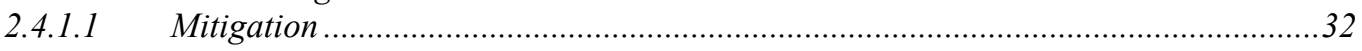

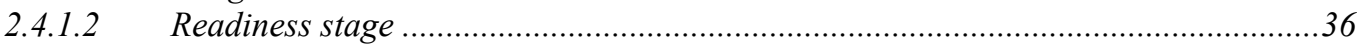

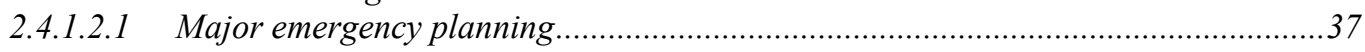

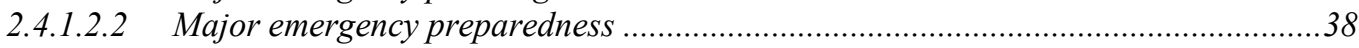

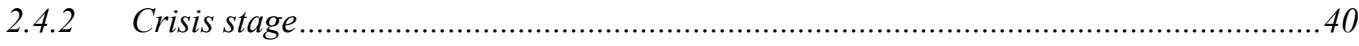

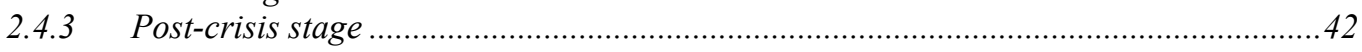

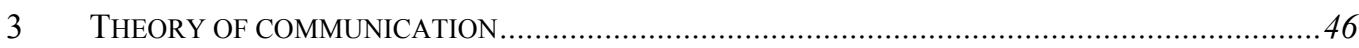

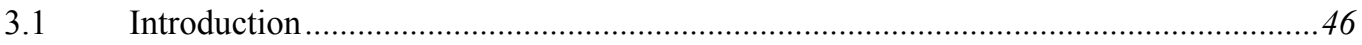

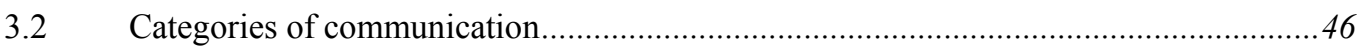

Components of communication.......................................................................... 47

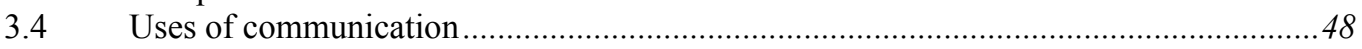

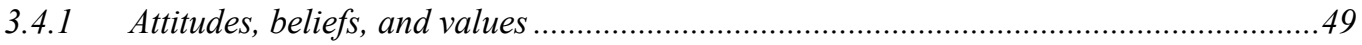

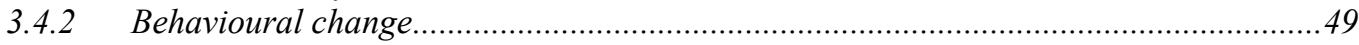

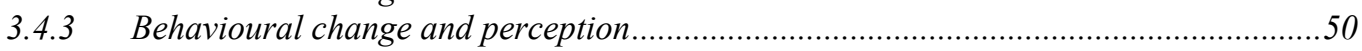

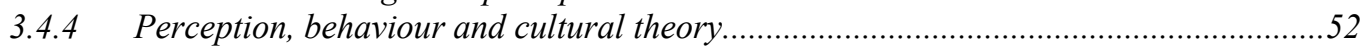

3.5 Developments in communication theory .......................................................5

3.5.1 Building on the rhetorical tradition - propaganda and persuasion............................55

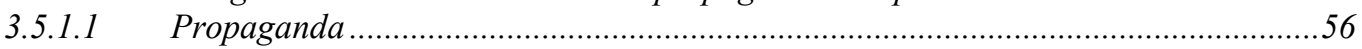

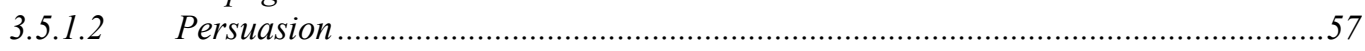

3.5.1.3 Other contributions of Lasswell, Lazarsfeld and Schramm ....................................58

3.5.1.4 Developmental communication ................................................................. 
3.5.1.5 Participatory communication ..............................................................................60

3.5.1.6 Move towards relational communication ............................................................61

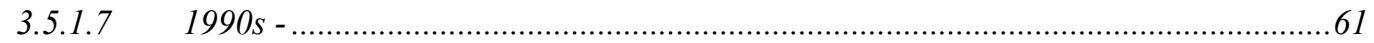

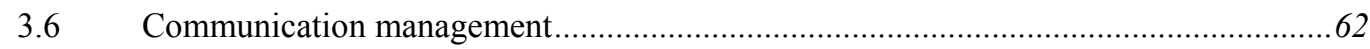

3.6.1 Models of communication management ....................................................................63

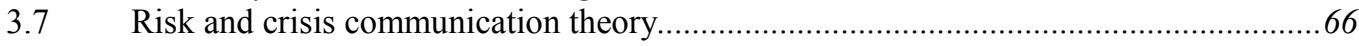

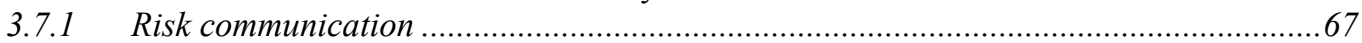

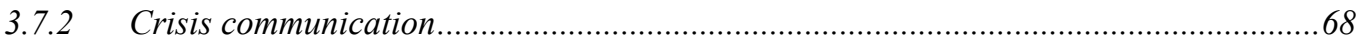

3.7.3 Crisis and emergency risk communication ..............................................................6 68

3.7.4 Evolution of risk and crisis communication ...............................................................69

3.7.5 Models of risk and crisis communication ................................................................... 72

4 STRATEGIC MANAGEMENT OF COMMUNICATION ....................................................... 75

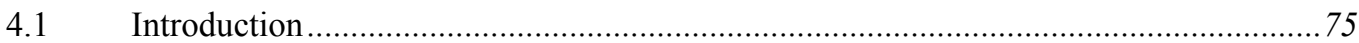

4.2 Overview of strategy and strategic management.................................................76

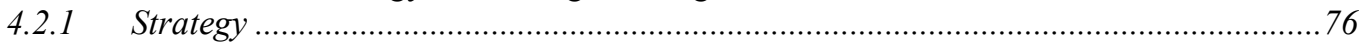

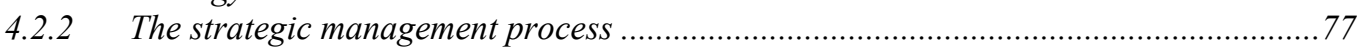

4.2.3 Strategic versus tactical approaches....................................................................... 80

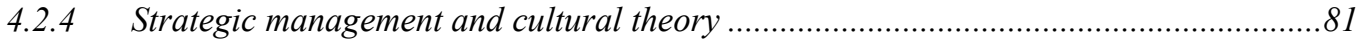

4.3 Strategic communication management.............................................................. 81

4.3.1 Tactical communication management ......................................................................83

4.3.2 A model of strategic communication management ...................................................84

4.4 Key elements involved in strategic communication management.............................8 87

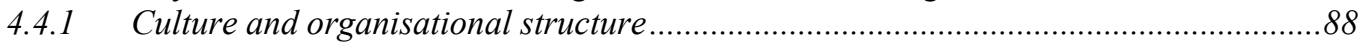

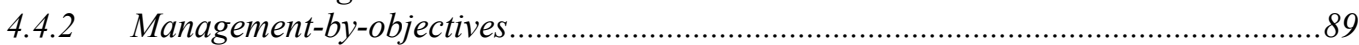

4.4.3 Strategic communication planning ....................................................................... 90

4.4.4 Strategic communication research .................................................................. 91

4.4.5 Strategic communication evaluation ..................................................................92

4.4.6 Building relationships .................................................................................. 93

4.4.7 Broad based deliberation ................................................................................94

4.4.8 Strategic communication knowledge: (Formal education and experience) ..................95

4.5 Strategic communication management during a major emergency situation ...............95

5 STRATEGIC PRINCIPLES AND THE MAJOR EMERGENCY COMMUNICATION FRAMEWORK ..... 100

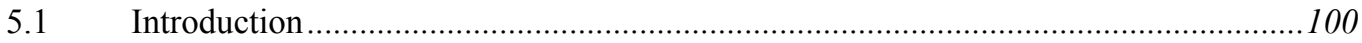

5.2 The major emergency communication and information team (MECIT) ....................101

5.3 Six strategic major emergency communication principles ..................................102

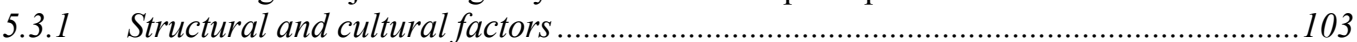

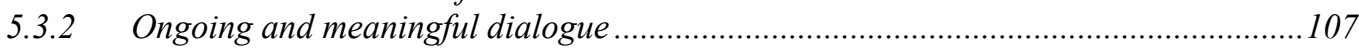

5.3.3 Adopting a scientific approach to communication ................................................110

5.3.3.1 Sound knowledge base .................................................................................... 111

5.3.3.2 Ongoing communication research ................................................................... 113

5.3.3.3 Ongoing communication evaluation.............................................................. 114

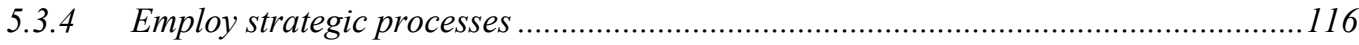

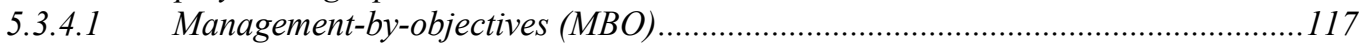

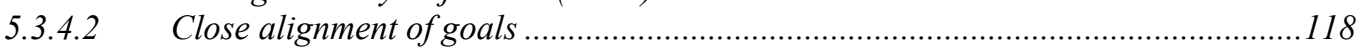

5.3.4.3 Engage in strategic communication planning .................................................... 120

5.3.4.4 Engage in strategic decision-making ........................................................ 122

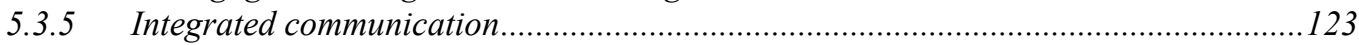

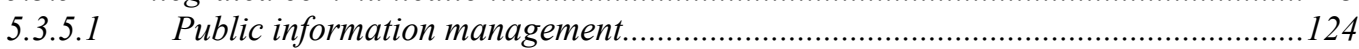

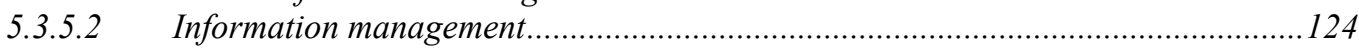

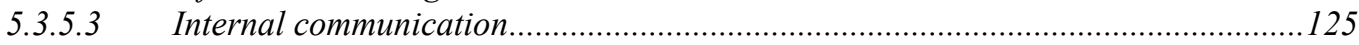

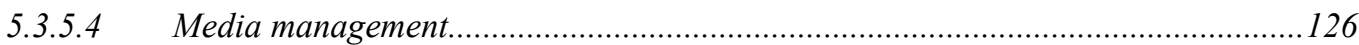

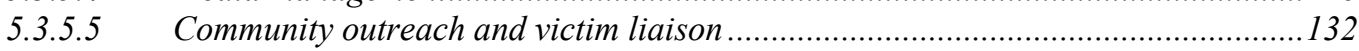

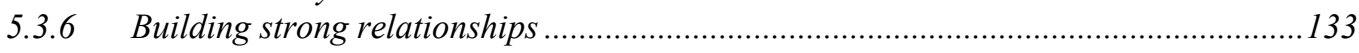

5.3.6.1 Developing and maintaining trust and credibility ..........................................136

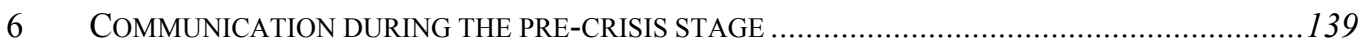

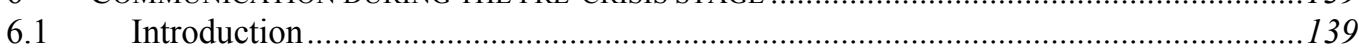

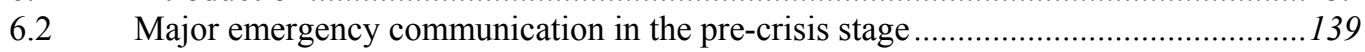


6.3 Higher-level pre-crisis major emergency communication management .....................140

6.3.1 Selection of senior communication manager/leader of the MECIT..........................140

6.3.2 Selection of the MECIT ............................................................................................ 141

6.3.3 Preliminary goal setting........................................................................................ 142

6.4 Pre-crisis stage tactical level ................................................................................... 143

6.4.1 Prevention during the pre-crisis stage ....................................................................... 143

6.4.1.1 The early warning detection role ........................................................................... 143

6.4.1.2 The building of strong stakeholder relationships and the fostering of alliances.....144

6.4.2 Communication preparation during the pre-crisis stage of a major emergency.......... 145

6.4.2.1 Conduct a detailed major emergency communication analysis.............................145

6.4.2.2 Prepare comprehensive communication goals and objectives ...............................146

6.4.2.3 Establishing communication approach parameters .............................................. 147

6.4.2.4 Redetermine relevant stakeholders and publics .............................................. 149

6.4.2.5 Message and information formulation............................................................... 150

6.4.2.6 Educating stakeholder groups ..................................................................... 150

6.4.2.7 Major emergency response communication plan (MERCP). ...............................154

6.4.2.8 Major emergency communication exercises and training ..................................155

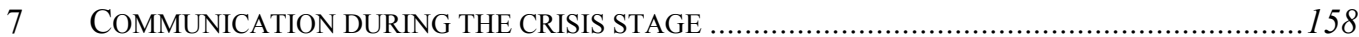

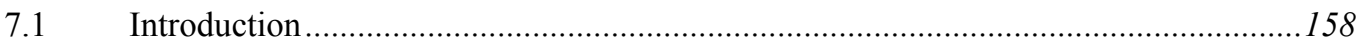

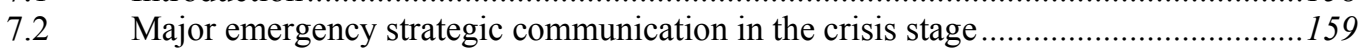

7.2.1 Early identification of imminent major emergencies...............................................159

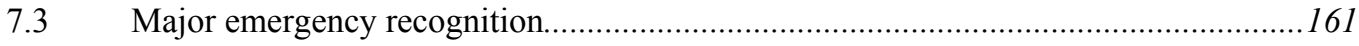

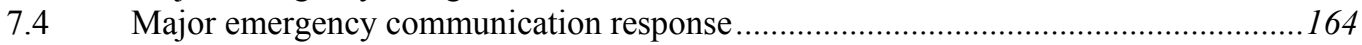

7.5 Communication with stakeholders and affected communities ..................................166

7.5.1 The crisis stage communication process ............................................................... 167

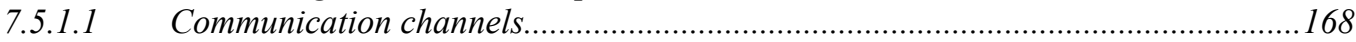

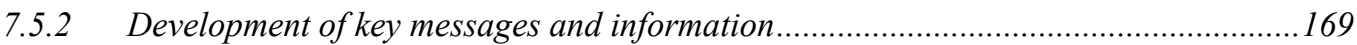

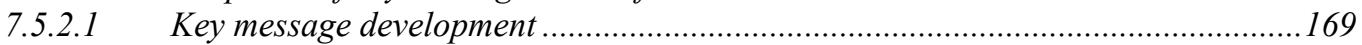

7.5.2.2 Communication research ............................................................................... 170

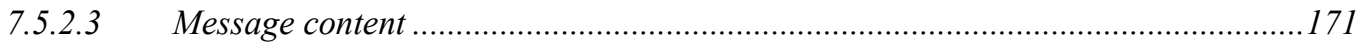

7.5.2.4 Testing key messages .................................................................................... 172

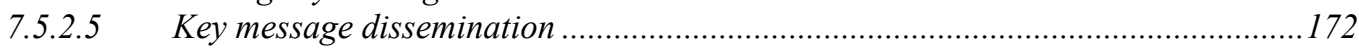

7.5.2.6 Empathy for affected publics ...................................................................... 173

7.5.3 The major emergency public warning process .................................................. 173

7.5.3.1 Issuing public warnings, alarms and instructing information ................................174

7.5.4 Likely behaviour of affected publics .................................................................. 178

8 COMMUNICATION DURING THE POST-CRISIS STAGE ................................................. 180

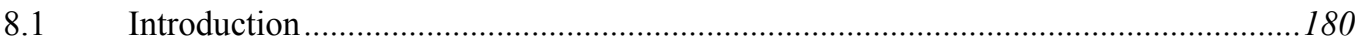

8.2 Major emergency strategic communication in the post-crisis stage ..........................181

8.3 Communicating with stakeholders and affected communities .................................. 182

8.3.1 Dealing with information demands ................................................................... 182

8.3.1.1 How to deal with information demands .................................................... 183

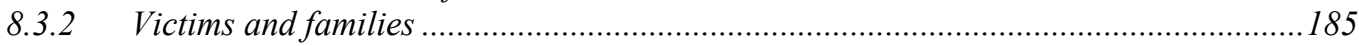

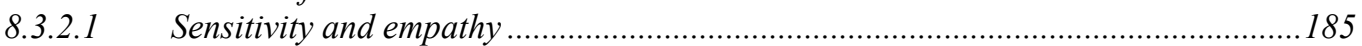

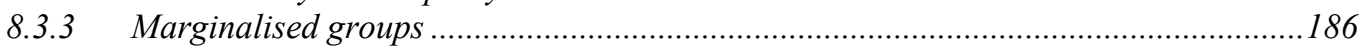

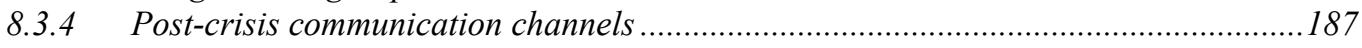

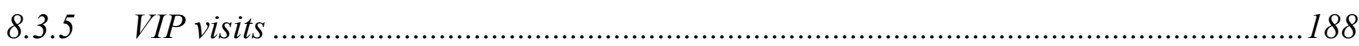

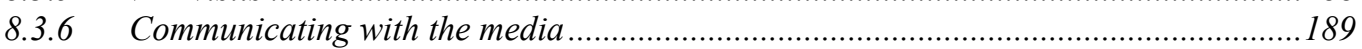

8.3.7 Communicating with major emergency responders and their families .............................190

8.4 Communicating business continuity management (BCM) ..................................191

8.5 Improvement of the major emergency communication strategy and plan..................192

8.5.1 Evaluation of all elements of the communication process...........................................193

8.5.2 Revise communication strategy and plan, and implement changes............................196

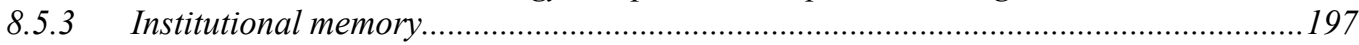

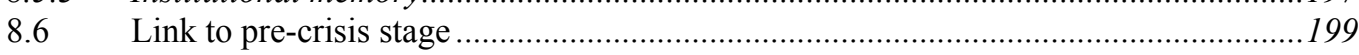

9 STRATEGIC COMMUNICATION MANAGEMENT IN THE UK, USA AND SWEDEN.....................202

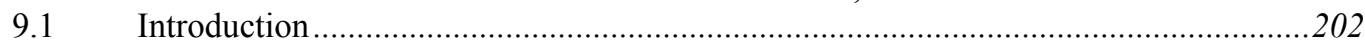




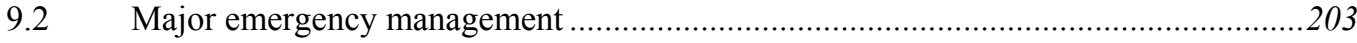

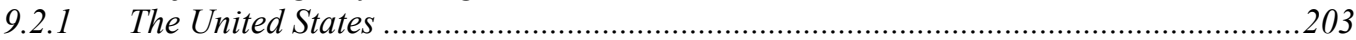

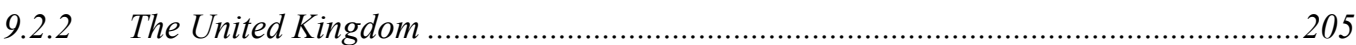

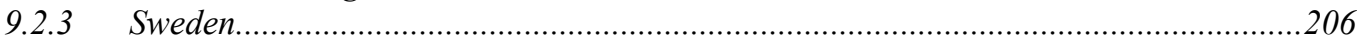

9.3 Major emergency strategic communication..............................................................208

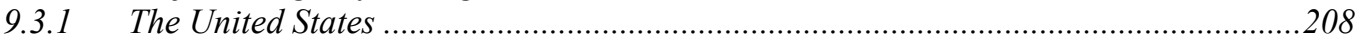

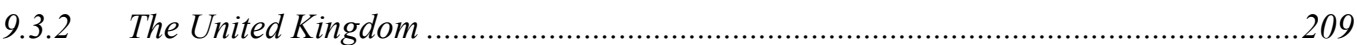

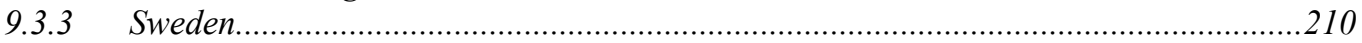

9.4 Comparative analysis ............................................................................................2 211

9.4.1 Strategic communication management and planning................................................211

9.4.2 Integrated communication.......................................................................................2 213

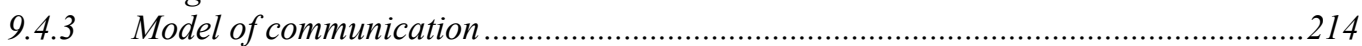

9.4.4 Broad-based deliberation (meaningful engagement with publics) .............................215

9.4.5 Management-by-objectives............................................................................2 217

9.4.6 Senior communication practitioner involved in decision-making at the highest level .217

9.4.7 Knowledge base - professional education and experience ........................................218

9.4.8 Major emergency communication research ..............................................................219

9.4.9 Major emergency communication evaluation .......................................................220

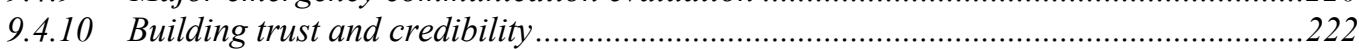

9.4.11 Integrated communication training and exercising ................................................222

10 STRATEGIC COMMUNICATION MANAGEMENT IN IRELAND.............................................224

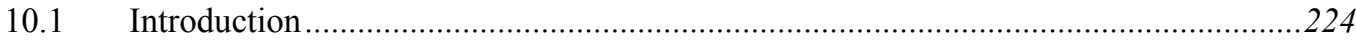

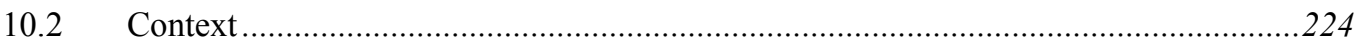

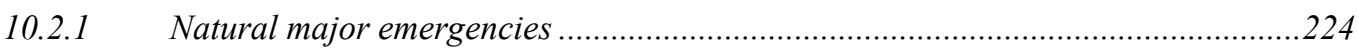

10.2.2 Anthropogenic major emergencies ..................................................................226

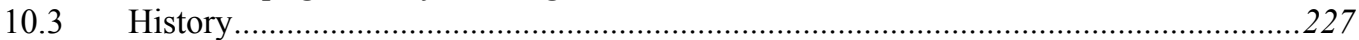

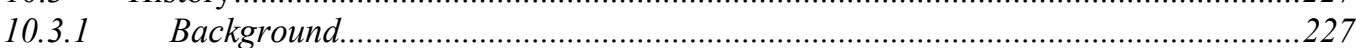

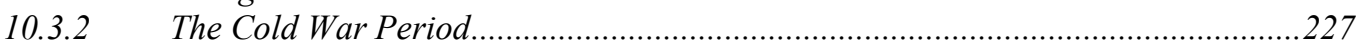

10.3.3 The Irish government's first significant public information effort.........................228

10.3.4 The first comprehensive major emergency plans.............................................229

10.3.5 Approach to communication management in the 1984 Framework .......................230

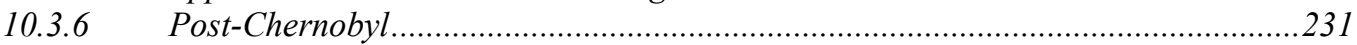

10.3.7 Major emergency management in the new century .........................................231

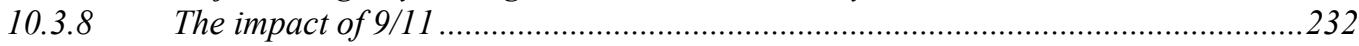

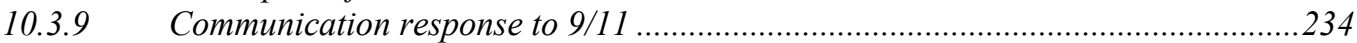

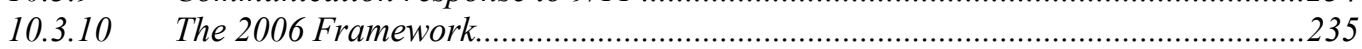

10.3.11 Major emergency public information and awareness campaign 2005-2008.........242

10.3.12 Communication initiatives by other response agencies ....................................24

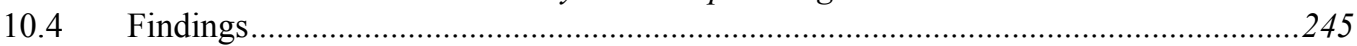

10.4.1 Major emergency management ...................................................................2. 245

10.4.2 Major emergency communication management .................................................2.249

10.4.2.1 Elements of strategic communication management ..........................................254

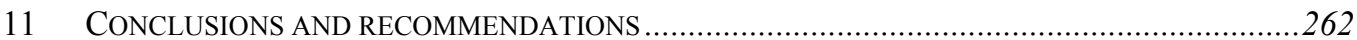

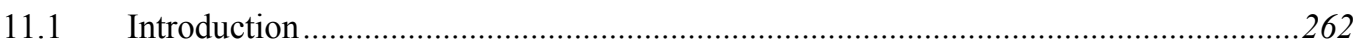

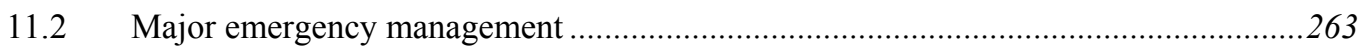

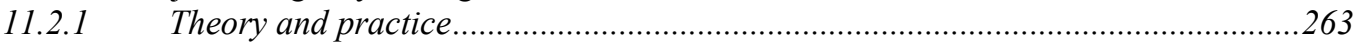

11.2.2 Revision and evaluation of major emergency structures and plans ...........................264

11.2.3 Major emergency legislation .....................................................................265

11.2.4 Major emergency oversight ........................................................................266

11.3 Major emergency management - Irish context .............................................266

11.3.1 Advances in major emergency theory and practice ................................................266

11.3.2 Whether the 2006 Framework reflects best-practice? .........................................269

11.3.3 Statutory status of the 2006 Framework ..........................................................270

11.3.4 Coordination and oversight role .................................................................271

11.4 Major emergency communication - International context......................................2273

11.4.1 Improvements in the theory and practice ....................................................273

11.4.2 Approach major emergency communication strategically ...................................274

11.4.3 Approach communication scientifically......................................................2. 276 
11.4.4 Integrated Communication ..................................................................................2 277

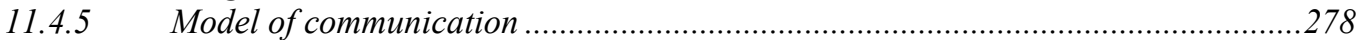

11.4.6 Meaningfully engage the public............................................................................2.

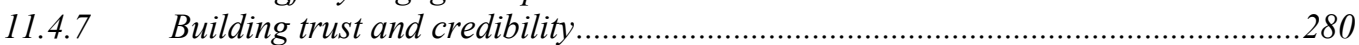

11.4.8 Integrated communication training and exercises...............................................28

11.5 Major emergency communication management - Irish context.............................281

11.5.1 The importance of major emergency communication .............................................281

11.5.2 Appreciation of major emergency communication ................................................282

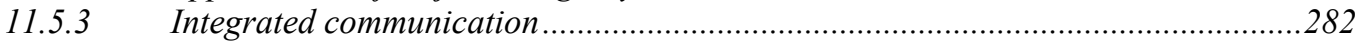

11.5.4 Long-term communication planning...........................................................28

11.5.5 Departmental and agency communication practitioners.......................................224

11.5.6 Centralised guidance structure .........................................................................28

11.5.7 Greater transparency and dialogue with members of the public...........................286

11.6 Key recommendations for Ireland ....................................................................28

Bibliography 290

$\begin{array}{ll}\text { Appendices } & 332\end{array}$

Appendix A Details of those who participated in the research

Appendix B A recommended outline structure of a major emergency response communication plan (MERCP)

Appendix C A copy of the self-administered questionnaire completed by officials in the United Kingdom, Sweden and the United States

Appendix D A copy of the semi-structured questionnaire used as a guide during interviews with major emergency communication officials in Ireland 


\begin{abstract}
This study asks if a strategic approach to the management of communication can assist and support the overall operational commander in effectively managing a major emergency situation. Communication in this regard includes important strategictactical functions that need consideration when the community faces significant loss of life or damage, and incorporates tasks such as media management, public information, the raising of public awareness, internal communication, public affairs, community relations, and survivor and victim relations. Encompassing these functions are a number of overarching principles that, when adopted, allow the entire process to be approached from a strategic rather than a tactical viewpoint. The study demonstrates how an efficient, strategically focused communication team can assist the operational commander in managing a major emergency situation.
\end{abstract}

The dissertation explores principles and practices for strategic communication management for major emergencies. It recommends a particular framework that is underpinned by six strategic communication principles. The framework was developed taking into account relevant theories and models of strategic communication and management, augmented by detailed primary and secondary qualitative deductive research. Research in this regard included reading not only the academic literature but also a broad range of official Irish and other documentation, including reports, manuals, guidance materials and legislation. Original primary research was undertaken to ascertain how a number of countries, regarded as leaders in this field, approach this subject and detailed interviews were conducted with responsible officials in Sweden, the United Kingdom and the United States. As relatively little data exists on the subject of major emergency strategic communication in the Irish context, in-depth interviews were also conducted with key individuals involved in major emergency management in Ireland.

The study demonstrates that the strategic communication function is increasingly a critical success factor in major emergency management internationally. It also shows that, while major emergency communication initiatives have been undertaken in Ireland, the Irish approach is somewhat fragmented and tactical in nature. 
Figure 2.1: Shifts in major emergency management

Figure 2.2: Composite major emergency life cycle

Figure 2.3: Risk management framework

Figure 3.1: Mixed-motives model of communication

Figure 4.1: Strategy formation activities

Figure 4.2: Strategic communication management framework

Figure 4.3: Major emergency communication management life cycle

Figure 5.1: Proposed framework for strategic major emergency communication management - overview

Figure 5.2: The importance of trust and credibility to major emergency communication

Figure 6.1: Major emergency communication management pre-crisis stage

Figure 7.1: Major emergency communication management crisis stage

Figure 8.1: Major emergency communication management post-crisis stage

Figure 8.2: Major emergency communication learning cycle 


\section{Lexicon of Acronyms}

\begin{tabular}{|c|c|}
\hline CBRN & Chemical, Biological, Radiological or Nuclear \\
\hline $\mathrm{CCS}$ & Civil Contingencies Secretariat (UK) \\
\hline $\mathrm{CDC}$ & Centers for Disease Control (US) \\
\hline CIMC & Communication and Information Management Centre \\
\hline $\mathrm{COC}$ & Cabinet Office Communications (UK) \\
\hline DAFF & Department of Agriculture, Fisheries and Food (Irl) \\
\hline DEHLG & Department of Environment, Heritage and Local Government \\
\hline DF & Defence Forces (Irl) \\
\hline DHS & Department of Homeland Security (US) \\
\hline DoD & Department of Defence (Irl) \\
\hline EOC & Emergency Operations Centre \\
\hline FEMA & Federal Emergency Management Agency (US) \\
\hline $\mathrm{GCN}$ & Government Communication Network (UK) \\
\hline GIS & Government Information Services (Irl) \\
\hline GS & Garda Síochána (Irish police service) \\
\hline HSE & Health Services Executive (Irl) \\
\hline ICT & Information and communication technology \\
\hline IEM & Integrated Management System \\
\hline IDWG & Inter-Departmental Working Group (Irl) \\
\hline $\mathrm{JIC}$ & Joint Information Centre \\
\hline $\mathrm{MBO}$ & Management-by-Objectives \\
\hline MECIT & Major emergency Communication and Information Team \\
\hline MERCP & Major Emergency Response Communication Plan \\
\hline MLO & Media Liaison Officer \\
\hline NEPNA & National Emergency Plan for Nuclear Accidents (Irl) \\
\hline NSC & National Security Committee (Irl) \\
\hline NSG & National Steering Group (Irl) \\
\hline NUI & National University of Ireland \\
\hline NWG & National Working Group (Irl) \\
\hline OEP & Office of Emergency Planning (Irl) \\
\hline OPW & Office of Public Works (Irl) \\
\hline PID & Project Initiation Document \\
\hline PRA & Principal Response Agencies \\
\hline RPII & Radiological Protection Institute Ireland \\
\hline SEMA & Swedish Emergency Management Agency \\
\hline \multirow[t]{2}{*}{ SPF } & Styrelsenfor Psykologiskt Forsvar \\
\hline & (National Board of Psychological Defence, Sweden) \\
\hline TD & Teachta Dála (member of the Irish parliament) \\
\hline
\end{tabular}




\section{Acknowledgements}

I would like to thank all who contributed to this research project over the past four years. A special word of thanks to Prof. Colum Kenny for his guidance, advice, support, encouragement and patience throughout this long journey. I would also like to thank the many individuals, both in Ireland and abroad, who participated in the research and shared their experiences, thoughts and insights with me. Without your assistance this research would not have been possible. Finally, and most importantly, to my wife Elaine, who has been forced to live with tsunami, hurricanes, earthquakes and all manner of pestilence and plague for the past four years; a thousand thanks for your patience and support. 


\section{INTRODUCTION}

\subsection{Overview}

Mankind has always faced the threat of large-scale death and destruction from disastrous situations. Mention of Pompeii, Krakatoa, Chernobyl, Banda Aceh and New Orleans evokes powerful images for many people. Despite all the advances in technology, disasters affect, and will continue to affect, whole communities in many significant ways. Research indicates that the number of people at risk from extreme events continues to grow at an alarming rate (Bouwer et al. 2007: 753). This includes threats not only from traditional geophysical events such as earthquakes and floods, but also from anthropogenic major emergencies such as the release of toxic substances, major transport accidents and terrorist related incidents. For example, in early May 2008, two significant disasters occurred in Asia leaving many thousands dead and even more homeless. On May $2^{\text {nd }}$, in Mynamar, Cyclone Nargis struck the Irrawaddy Delta region causing the deaths of approximately 80,000 people (BBC 2008(a)). On May $12^{\text {th }}$, in the Sichuan province of China, a massive earthquake killed more than 55,000 people (BBC 2008(c)). It is not surprising to find societies devoting attention to major emergency preparedness and planning, and training frontline agencies to respond effectively. This involves providing planners and responders with requisite skills in a number of critical competencies, including leadership, strategic management and planning, risk management, logistics, mitigation, information management, information and communications technology (ICT), and business continuity management.

In recent times, major emergency planners have identified an additional key competency that is increasingly recognised as a critical success factor in major emergency management, 'the strategic risk and crisis communication function'. It is recognised that the public requires a level of awareness and knowledge regarding lifethreatening risks that they face on a daily basis. Their involvement in decisions that are taken in order to protect them against these risks can be beneficial. Once a major emergency occurs, the public requires immediate and clear warnings and alarms to 
allow them to take adequate precautions. Sometimes these warnings are not possible and the events are so shattering that affected publics need effective, clear and compassionate information and reassurance in order to help them to cope and to begin to build a sense of order and understanding in their lives. Such major emergency communications are at the heart of this dissertation.

\subsection{The research question}

The principal research question at the heart of this study asks if a strategic approach to the management of communication is of benefit throughout a major emergency situation. The dissertation seeks more particularly to determine whether a communication team, following particular strategic principles of communication management and adopting a strategic approach, can assist and support the overall operational commander in effectively managing a major emergency situation. The substantive study centres on the relevant sub-fields of communication and specifically on strategic communication management. However, in order to answer adequately the research question, an analysis of the literature relating to two additional fields of endeavour is conducted, major emergency risk and crisis management, and strategic management and planning.

In addressing the research question, the concepts, theories and models of the relatively recent discipline of major emergency risk and crisis management will be examined. Of considerable interest in this regard is how communication flows within and throughout major emergency response organisations and how communication assists and supports the various major emergency management functions. Central to this research is an analysis of the theories, principles and models of communication and their relationship to the major emergency context. Additionally, the study involves an analysis of communication management itself, including the planning, execution and evaluation of an organisation's communication with external and internal publics, in order to determine its role in a major emergency situation. As the substantive study centres on the strategic aspects of communication management, it is important to comprehend the theory and principles of the well-established discipline of organisational strategic management and planning. An analysis such as this allows 
for a greater understanding of how this discipline has evolved over the past fifty years, together with a rationale for these changes. Additionally, it is important to determine whether the theory of strategic management and planning has an application within the context of major emergency communication, and to identify a number of key strategic principles that can be used in the development of a core conceptual major emergency communication framework. These are presented in Chapter 5.

\subsubsection{Conceptual strategic major emergency communication framework}

As will be seen throughout the dissertation, the study of major emergency communication management is supported by a significant amount of literature. However, much of this literature is somewhat fragmented, in that particular works address specific aspects of the overall area. This means that it has proven impossible to identify a single comprehensive, integrated strategic major emergency communication framework that can serve as a guide, both strategically and operationally, for practitioners during all stages of a major emergency event. Consequently, one of the objectives of this study is to propose a core conceptual framework that can be of value as a guidance tool for practitioners, not least in the Irish context, and that can, additionally, serve as a basis for academics engaging in further research in this field.

The framework, as presented later in Chapter 5, is considered from two distinct perspectives, one strategic and the other strategic-tactical. It is intended to represent the strategic level using a number of overarching principles that are designed to encompass, underpin and guide the many communication functions undertaken by the practitioner throughout the three stages of the major emergency life cycle. In addition to the strategic benefits of doing so, it is intended that the conceptual framework should serve as a useful operational guide for communication practitioners throughout a major emergency event. Consequently, the conceptual framework includes a strategic-tactical level, involving a series of steps that should be considered in order to manage effectively the communication function throughout the three stages of a major emergency. 
The proposed core conceptual major emergency framework guiding the substantive study is drawn from an analysis of the literature from the relevant sub-fields of communication, strategy management and major emergency risk and crisis management, and supported by data acquired from Irish and international experts in the field of major emergency management and communication. These international experts are chosen from countries that are considered leaders in the field of major emergency management and communication practices.

\subsubsection{Best-practice and best-international-practice}

The terms 'best-practice' and 'best-international practice' are employed occasionally throughout this dissertation to refer to the use of a communication methodology and repeatable procedures and practices that appear to optimise all elements of the major emergency communication function. In using the terms to identify practices that are at present adopted by relevant authorities in some democratic states that have considerable experience in emergency planning and management (Sweden, the United Kingdom and the USA particularly), the author recognises that such terms are relative. Thus, the term 'best-practice' should not be understood to imply that major emergency communication management has reached a level that cannot be improved upon. Recent major emergencies, such as Hurricane Katrina and 9/11, have demonstrated that major emergency communication management is far from perfect and that lessons continue to be learned. Practice continues to evolve and improve.

Countries that engage in best practice major emergency communication management provide resources to accumulate and apply knowledge, thereby determining more likely than otherwise what is working and not working in different situations and contexts. In order for their major emergency communication management to be considered as best practice, it is necessary for government agencies to seek to improve it through the continuing process of learning, feedback, reflection and analysis. Other key areas examined are as follows. 


\subsubsection{The approach taken by the Irish government}

This study will determine the extent to which the Irish government is approaching major emergency communication management from a strategic perspective. It analyses the structures and guidelines that have been put in place to progress and support the major emergency management effort and the supporting communication agenda. The research examines the attitudes of senior major emergency planners towards adopting a strategic communication approach and their understanding of the principles in this regard. It also considers both the major emergency communication initiatives that are currently underway as part of the Major Emergency Development Plan (2006-2008) and those being planned, in order to ascertain the extent to which they are strategic in their approach.

\subsubsection{Link to tactical major emergency communication management}

As this dissertation is one of only a few comprehensive studies of major emergency communication management that examines the subject from both a strategic and a practical perspective, it is considered useful for practitioners of the discipline, and scholars who wish to undertake further research in this area. The proposed framework in this study places significant emphasis on communication at a strategic level. However, it also creates a link to the tactical (practical) level by introducing a series of strategic-tactical steps that communication practitioners can follow for each stage of the major emergency life cycle, while still adhering to the strategic principles.

\subsubsection{Reveal the available literature}

As this dissertation will demonstrate, it is difficult to source useful literature in Ireland on the subject of major emergency communication and related disciplines. Hence, it is considered beneficial to introduce the reader to as broad a base of literature as possible within each of the diverse subject areas examined as part of this research. Consequently, key subject areas are supported with more citations that one might otherwise expect, together with an expansive bibliography. This provides a useful reference list for further research in this subject area. 


\subsection{Scope and limitations of the study}

As stated earlier, this study examines the strategic communication management process throughout all three stages of a major emergency life cycle - pre-crisis, crisis and post-crisis. In this regard, the term 'communication' does not refer to technical communication systems or processes, such as response organisations' radio equipment. While the free flow of data through such systems is an extremely important factor for successful major emergency communication, it is outside the scope of this research and mentioned in passing only.

In this study, the emphasis is on the communication management process for a major emergency situation, and for more serious events such as disasters and catastrophes. Consequently, an analysis of routine adverse events managed by response agencies, such as localised fires and road traffic accidents, is outside the scope of this research. Additionally, the subject of organisational crisis communication is excluded.

The subject of risk communication is an extremely complex and specialised field of endeavour that includes sub-disciplines such as the psychology of risk, and social and cultural aspects of risk perception. While this subject is described in some detail at different stages in the dissertation, a comprehensive analysis of the subject is outside the scope of the research.

Additionally, in order to introduce the reader lucidly to as many of the diverse concepts and elements associated with strategic major emergency communication management as possible, it was thought prudent to present the dissertation in a report format rather than in a less formal free-flowing narrative style. This format was agreed with the supervisor.

\subsection{Significance and contributions of the study}

This dissertation is important as it contributes greatly to the limited number of studies on the subject of strategic major emergency communication management. The study will show that very few comprehensive frameworks dealing with this discipline exist, 
particularly ones that incorporate both strategic and practical levels. This dissertation provides a greater understanding of this complex subject for practitioners of the discipline. It is the first research to be conducted into the area of strategic major emergency communication in the Irish context.

Consequently, it is hoped that this research will be of interest to the Irish government and to Irish major emergency planners and communication practitioners who are currently engaged in progressing a redevelopment of the entire emergency planning function. The results of this study will also be useful to scholars of communication and to practitioners of risk, crisis and major emergency communication internationally.

\subsection{Terminology and definitions}

An examination of both communication and major emergency literature reveals two related disciplines, replete with terminology and definitions, many of which are used interchangeably. Some terms are also part of everyday discourse. This can result in members of the general public and certain stakeholders legitimately adopting a different understanding and usage of particular terms. Inconsistencies in major emergency language are likely to create a certain amount of confusion for the major emergency responder and communication practitioner.

In order for major emergency communication to be successful, it is important that relevant terminology is clearly understood by all and that it is applied consistently in both theory and practice. According to Quarantelli (1995: 224):

A main reason why we need clarification is because otherwise scholars who think they are communicating with one another are really talking of somewhat different phenomena.

\subsubsection{Crisis terminology and definitions}

In common parlance, the misuse of the terms 'crisis', 'emergency' and 'disaster' does not create any significant problems. However, the imprecise use of these terms by major emergency responders can result in either an inadequate, or else an excessive, 
response. It is important to be able to differentiate clearly between the categories of crisis-related events in order to allow response agencies to know exactly the type of situation that they are dealing with. Those delivering the MSc in Risk, Crisis and Disaster Management at the University of Leicester state:

The failure to distinguish between emergencies, crises and disasters also raises questions about the validity of any synthesis between theory and practice (Scarman Centre 2004: 2-10).

Additionally, Drabek and Hoetmer (1991: xviii) emphasise the importance of being able to establish the progression from incident to crisis to emergency to disaster for the purpose of potentially 'scaling up' a response.

For simplicity sake, the following terms are categorised with regard to their position in the crisis life cycle, beginning with the period prior to the actualisation of a crisis event, the pre-crisis stage. Descriptions of terms are also given in ascending order of seriousness, from the least serious to the most serious in terms of potential death and damage. While the term 'crisis' appears on the less serious end of the spectrum, it is also used as a generic noun to describe all categories of negative events that are in the process of occurring or have actually happened. Consequently, major emergencies and disasters can also be considered crisis events. However, using the term crisis as a generalised typology does not provide adequate clarity for effective response agency action and will be used to a minimum throughout this dissertation. Terms defined below will be discussed in more detail in Chapters 2 and 3 . 
Prior to a crisis event occurring

A 'hazard', according to Coppola (2007: 2), is:

A source of danger or an extreme event that has the potential to affect people, property and the natural environment in a given location.

Closely associated with a hazard is 'risk', which refers to the awareness that a latent (conceivable but long-term), potential (statistical in the sense that its probability has been calculated) or acute (imminent) threat or hazard exists and may result in events that adversely impact on individuals, groups or society at large (Nordlund 1994: 11).

\section{Following the occurrence of a crisis}

At the least serious end of the spectrum of seriously damaging events is an 'incident' which, according to Coombs (1999: 3), is 'a minor, localised disruption that does not disrupt the organisation's routine'. At the next level of seriousness is a 'crisis'. Millar and Smith (2002:1) describe a crisis as ' ... a significant disruption of an organisation's normal activities that stimulates extensive media coverage and public scrutiny'. Nohrstedt believes that 'crises' can be defined on both an individual or collective level (2000: 137).

An event that is more serious than a crisis can be termed an 'emergency'. Drabek and Hoetmer (1991: xvii) define an emergency as:

A routine adverse event that does not have community wide impact or does not require extraordinary use of resources or procedures to bring conditions back to normal.

An 'emergency' generally affects only a small portion of the community and response organisations do not need to draw on outside resources or help to respond. However, when an emergency situation 'overwhelms society's problem-solving resources and devastates the day-to-day lives of a great many people', Darrell (2003: 6) describes this as a 'major emergency'. The management of a major emergency situation is most likely beyond the normal capabilities of the response organisation in the affected area and requires the activation of additional procedures to ensure an 
effective, coordinated and integrated response (Government Task Force on Emergency Planning (2006(b): 13).

When a 'major emergency' is especially serious it can be considered a 'disaster'. A disaster is defined as:

A serious disruption of the functioning of society, causing widespread human, material or environmental losses, which exceeds the ability of the affected society to cope using only its own resources (DHA 1992: 15).

Disasters are often classified according to their source, natural or man-made. By their very nature, disaster situations are likely to require either national or international external assistance.

While many theorists, including Nohrstedt (2000: 137) and Lindell, Prater and Perry (2007: 2), equate 'disaster' situations with 'catastrophes', Drabek and Hoetmer (1991: xviii) describe a catastrophic situation as even more serious than a disaster. They characterise a 'catastrophe' as a 'disaster' that 'affects an entire nation and requires extraordinary resources and skills for recovery' - some of which must come from other nations. It is in this latter, narrower, sense that the term 'catastrophe' will be used by the author of this dissertation.

\section{Examples of the misuse of terminology}

Emergency services themselves differ in how they interpret types of emergency situation. For example, both the 1980 Buttevant rail crash (a rail crash that occurred in Buttevant, Co. Cork on August $1^{\text {st }} 1980$, resulting in the deaths of 18 people) and the 1981 Stardust fire (a fire that occurred on February $14^{\text {th }} 1981$ in Artane in Dublin in which 48 people died), are incorrectly described as 'disasters'. Both of these devastating incidents are better termed 'emergency' situations. As Nohrstedt (2000: 137) points out, terms such as 'disaster', 'crisis', 'catastrophe' and 'major emergency' have a particular meaning and 'are not synonymous or interchangeable'. Drabek and Hoetmer (1991: xviii) explain: 
In a sense, therefore, what constitutes a disaster depends on the community itself: the jurisdiction's size, its resource base, and its experience with a particular hazard.

\subsubsection{Other terms}

Stakeholders, public and publics

Grunig (1992: 127) describes the general public as 'a contradiction in terms' because different groups form in response to particular problems that seldom affect everybody in the population. One of these groups is termed 'a stakeholder', which is a party that affects, or can be affected by, the actions of an organisation. As a widespread major emergency, such as influenza, can affect the entire population, it may be said that all members of the public are potential stakeholders for major emergency situations. Certain stakeholders can decide to engage in communication with the organisation as a unified collective and are termed 'publics' (Broom and Dozier 1990: 32).

\subsubsection{Communication terminology and definitions}

\section{Communication and communications}

The use of the terms 'communication' and 'communications' can be confusing. According to Theodorson and Theodorson (1969: 83), communication is the transmission of information, ideas, attitudes, or emotion from one person or group to another (or others) primarily through symbols (many more definitions of communication will be offered later in Chapter 3). James Grunig clearly differentiates the term communication from communications when he states:

To some people, perhaps to you, managing communications is only the management of messages and does not include such activities as research, counselling of management, and participation in strategic management. In the plural, 'communications' refers to messages. The singular 'communication' however, refers to a process (Grunig 2006: personal communication).

Throughout this dissertation the term 'communication' will be favoured over 'communications'. This term is more relevant to the core subject matter of this study, which is the process of strategic communication management. 


\section{Communication management and public relations}

Two further terms that generate considerable confusion are 'communication management' and 'public relations'. As is demonstrated later in Chapter 3, many theorists consider both disciplines to be equivalent. According to James Grunig (2006: personal communication):

The distinction between public relations and communication management is arbitrary... both involve the management of communication to cultivate relationships with strategic publics.

Consequently, throughout this study, both terms are considered equivalent and interchangeable. Nevertheless, for the sake of consistency, the term 'communication management' is adopted wherever possible.

\subsection{Methodology}

\subsubsection{The model}

The methodology adopted for this study involves both a detailed review of international data and primary qualitative deductive research. According to Denzin (1978: 28):

No single method ever adequately solves the problem of rival causal factors as each method reveals different aspects of reality...multiple methods of research must be employed.

Consequently, this study utilises a process of data 'triangulation' (Quinn-Patton 2002: 247), in which multiple research methods are employed. Central to this research is a considerable amount of document review. In the review of documents, a broad range of relevant texts, together with available official literature, including reports, manuals, guidance materials and any relevant legislation extant, are examined. This includes documentation from the US, the UK, Sweden and Ireland. The detailed document review is then supported by primary data gleaned from in-depth interviews, elite interviews, e-mail and telephone interviews, and a detailed questionnaire. 
As little data exists on the subject of major emergency communication in the Irish context, primary research proved of critical importance to this study. In this regard, two distinct categories of primary research were employed. Firstly, detailed in-depth and elite interviews were conducted with key individuals involved in major emergency management in Ireland. Secondly, comprehensive self-administered questionnaires were completed by three international major emergency communication practitioners and supplemented by follow-up telephone and e-mail interviews. In the choice of both categories of participants for the primary research, criterion sampling was the preferred sampling method, where individuals are chosen who meet some specific criterion - in this case expertise in particular areas of major emergency operations or communication management. There is a small number of such personnel in Ireland. Choosing participants in such a manner 'provides validity and depth to the research' (Quinn and Patton 2002: 243). In deciding the appropriate sample sizes for both categories of primary research, Quinn-Patton provides guidance stating:

The validity, meaningfulness and insights generated from qualitative inquiry have more to do with information richness of the cases selected than with sample sizes (Quinn-Patton 2002: 245).

From information-rich cases 'one can learn a great deal about matters of importance and are therefore worthy of in-depth study' (ibid: 242). According to Broom and Dozier, 'in-depth interviews take considerable time to conduct... as such they are generally limited to small, purposive samples' (1990: 146). Consequently, fourteen individual 'information-rich' interviews took place with nineteen people in Ireland, and comprehensive self-administered questionnaires were completed with single participants in the United States, the United Kingdom and Sweden.

\subsubsection{Ireland}

In selecting the list of participants for interview in the Irish context, it was useful first to examine the membership of both the major emergency National Steering Group (NSG) and the National Working Group (NWG). Both of these groups have been centrally involved in driving the major emergency planning effort in Ireland since 2006. Consequently, it was decided to conduct in-depth interviews with the heads of major emergency planning in the Garda Síochána (GS) and the Health Services 
Executive (HSE), the head of the Office of Emergency Planning (OEP) and the Government Press Secretary. Additionally, in-depth and elite interviews were conducted with the Minister for Defence, an Assistant Secretary of the Department of the Environment, Heritage and Local Government (DEHLG) an Assistant Secretary of the Department of Agriculture, Fisheries and Food (DAFF), and the Deputy Chief of Staff (Operations) of the Irish Defence Forces (DF).

In addition to the criterion sampling method, it was deemed useful to engage in some emergent sampling (Quinn-Patton 2002: 244), where new leads emerged during fieldwork. This sampling method was pursued to the 'point of redundancy', at which stage further interviews resulted in no new information forthcoming (Lincoln and Guba 1985: 202). Interviews in this regard took place with the Director of the Radiological Protection Institute of Ireland (RPII), the Director of Communications for the Garda Síochána, and an additional interview with a member of the Health Services Executive.

In order to gain a perspective external to the official major emergency planning effort, it was decided to conduct in-depth interviews with an independent risk and crisis management consultant, a former member of the Office of Emergency Planning, and also with the managing director of one of Ireland's leading public relations firms. These three interviewees have considerable knowledge of major emergency planning and management (for details of all of the interviews conducted see Appendix A).

The comprehensive interviews for the Irish participants were based around semistructured questionnaires, where the pre-determined order, or wording, of the questions could be changed to suit the role of the particular interviewee and the situation. According to Creswell and Plano-Clark (2007: 30):

In order to best obtain the perspectives from the participants, using reasonably open-ended questions, the researcher focuses on a single concept or phenomenon at one time and learns about this phenomenon in depth.

The framework for each individual questionnaire was structured around the different critical strategic elements of both the major emergency management and the major 
emergency communication process. Pursuing this type of framework, and opting for a qualitative dialectical form of research, it was envisaged that points could be expanded, issues debated and 'elaboration probes' pursued during the interviews (Quinn-Patton 2002: 375). However, cognisance was given to Broom and Dozier's (1990: 145) warning that, ' ...great care needs to be taken to ensure that any directing of the interview does not lead the interviewee away from his or her frame of reference'. However, as some of the interviewees displayed a limited knowledge of certain aspects of strategic major emergency communication management, it was occasionally necessary to expand on questions in the course of the interview, and to introduce 'neutralised prompts' (Deacon et al.1999: 64).

It was necessary to carefully evaluate the answers given. Campbell, cited in Bulmer, (2004: 215), discusses the problem of 'unfulfilled objectives' where the interviewee, for any number of reasons, attempts to 'evade' the desired answer. In order to ensure validity and confidence in the findings multiple data sources were summarised, collated and synthesised, including the data acquired from the two external consultants.

All of the interviews were recorded with the stated permission of the participants. The recorded information was then transcribed and edited. The edited text was sent back to the interviewees in order to ascertain that it fairly reflected their views. In some cases, requests were received for minor amendments to be made.

The analysis stage involved categorising the interview results into distinct segments based on the key elements under review. Once this process was completed, the data was transferred to a mind-mapping tool and a 'colour-coding' process employed in order to cross-reference inter-related elements. This process made it possible to develop themes that appear throughout the different categories of responses. Shank (2006: 148) describes this process as 'thematic analysis... which involves searching for patterns in data'. According to Creswell and Plano-Clark (2007: 29), the themes are then interrelated to form broad generalisations thereby allowing conclusions to be drawn. Analysing data such as this, according to Morse (1994: 25): 
Is a process of piecing together data and of making the invisible obvious, of recognising the significant from the insignificant and of linking seemingly unrelated facts.

\subsubsection{International comparison}

In order to compare the practices of strategic major emergency communication management in Ireland with best-international-practice, detailed research of international literature was undertaken. This included reference to relevant sub-fields such as emergency risk and crisis management, the multi-disciplinary fields of communication studies and management studies. The information from this secondary research was supported and confirmed by information gleaned from comprehensive self-administered questionnaires that were completed by three international major emergency communication practitioners and supplemented by follow-up telephone and e-mail interviews. In choosing the three participants, criterion sampling was the method used. In this regard, three countries were chosen that appear to be leaders and innovators in the development of major emergency theory and practice. Countries engaging in best international communication practice, as defined earlier in Section 1.2.2, include the United States, the United Kingdom, Sweden, Canada, and Australia.

Contact was established with the government agency responsible for major emergency management in each country, the Federal Emergency Management Agency (FEMA) in the US, the Swedish Emergency Management Agency (SEMA), and the Civil Contingencies Secretariat (CCS) in the UK. Each of these agencies proposed a senior communication practitioner to participate in the research.

In order to elicit adequate information from each of the countries it was decided to send a comprehensive self-administered questionnaire to the three participants, Daryl Madden in FEMA, Malin Modh in SEMA and Carol McCall in Cabinet Office Communications (COC) (see Appendix C). This allowed the participants time to consider their responses, and overcame any possible language barrier for the Swedish participant. Additionally, it was noted that Gibson and Hawkins, cited in Bulmer, (2004: 84), believe 'a questionnaire may produce substantially the same results as indepth interviews at a much smaller cost'. 
The questionnaire was of considerable length ( 80 questions). However, the three participants were informed well in advance that it would take approximately one hour and thirty minutes to complete. None of them expressed any reservations in this regard. In the construction of the questionnaire, it was deemed prudent to use both open and closed answer formats to 'take advantage of the specific benefits of each and to avoid any particular limitations' (Deacon et al. 1999: 76). As each participant was obliged to consider and answer questions across a number of critical areas, the closed response questions proved quicker to answer, thereby saving the respondent time. In order to make the respondent think about each question in turn, and to prevent a uniform and undifferentiated response, a number of different types of closed-response questions formats were utilised, including Likert scales, semantic differential formats, checklists, and ranking formats. Other open-response questions were posed for particular subject areas in order to 'elicit richer, more sensitive insights into the views and activities of the respondents' (Robson 2002: 291).

The three questionnaires were dispatched in March 2007. Two of the completed questionnaires were received at the end of May 2007 and the third received in January 2008. Following receipt of individual questionnaires, two follow-up phone calls were made to each of the participants in order to clarify a number of their answers and also to probe a number of issues further. These phone calls were limited to twenty minutes each and the interviews recorded with the permission of the participants. The information was then transcribed and compared with the data gleaned from the questionnaires. Additionally, a number of e-mails were exchanged with the participants in order to address specific points.

\subsubsection{Other research}

In the course of the research it proved useful to make personal contact with a number of highly regarded theorists in the field of strategic communication. This allowed for the gathering of additional information, the clarification of theoretical concepts and the reception of expert opinion. Consequently, e-mail communication was established with theorists such as Peter Sandman, Jody Lanard, James Grunig, Baruch Fischhoff, and Birgitta Darrell. In addition, some Irish academics were contacted by e-mail to 
explain particular events, including Dr John Tyrrell (typhoons) and Prof. Mike Williams (big freezes). The detail of these personal communications is contained in Appendix A.

\subsection{Overview of review of the literature}

As mentioned in the previous section, the methodology adopted for this study involves a considerable amount of secondary research, utilising a broad range of literature. This is considered important because in order to examine thoroughly the subject of strategic major emergency communication management, it is necessary not alone to have a comprehensive knowledge of the literature that supports this important subject but also to explore a number of analogous disciplines, including major emergency management, communication theory and strategy management. Consequently, it is necessary to analyse a number of forms of literature for each of these related disciplines, including text books, academic journals and papers, relevant legislation, guidance documents, protocols, reports and manuals, major emergency websites, and media reportage. Relevant documentation relates to a number of countries, including most particularly the USA, the UK, Sweden, Australia, New Zealand, Japan, India, Cuba, and Ireland.

It will be shown that some of the disciplines, such as communication theory and strategic management, are well served by an abundance of useful and informative literature. However, other important disciplines, including strategic major emergency communication management - particularly within the Irish context - do not have a solid base of literature to support them. The libraries of Dublin City University (DCU) and Dublin Institute of Technology (DIT) stock a number of useful texts on the subject of communications theory, while those of Trinity College Dublin and DCU include texts on risk and emergency management. However, generally speaking, holdings in Ireland on some aspects of the most relevant disciplines were limited both in nature and in number. Consequently, it was necessary to order or acquire especially quite a few works from abroad that could not be found already in Irish libraries. A visit to the United Kingdom Cabinet Office's library at the Emergency Planning College in Easingwold, York, proved a rich source of material for both major emergency management and major emergency communication. 
For the sake of presentation, this chapter is divided as follows, major emergency risk and crisis management, communication theory, organisational strategy and strategic communication management, and strategic major emergency communication management both internationally and within the Irish context. A critique of the most useful literature available and of how it contributes to the study is also provided. Additionally, gaps in the literature will be discussed.

\subsection{Organisation and structure of the thesis}

In order to deal with the research question adequately, this dissertation firstly reviews the literature from three core areas throughout Chapters 2, 3 and 4.

Chapter 2 provides an overview of much of the available literature that deals with the subject of major emergency management. The chapter begins with a review of major emergencies and how they can be categorised in terms of life cycles. There follows a description of the shifts that have taken place in the management of major emergency situations over the past three decades. Central to the entire chapter is a consideration of the different stages of major emergency management and the critical steps that managers have to take within each stage in order to deal with the adverse situation. As a detailed analysis of the discipline of major emergency management throughout all three stages is outside the scope of this dissertation, it is important to focus on providing a broad overview of the elements of each stage. However, as a central focus of this study is strategic communication planning and preparation, particular emphasis is placed on the pre-crisis stage of major emergency management. This includes a description of the risk management process, which is an important factor in determining how major emergencies are perceived by members of the public.

Chapter 3 provides the reader with an understanding of communication theory, including the models and elementary concepts that underpin the communication process. Such information provides the reader with a useful basis for comprehending the principles of strategic major emergency communication management. The chapter begins with an examination of the different categories of communication and follows by discussing traditional components such as message, sender and receiver. An important element of major emergency communication is then discussed, concerning 
the use of communication in influencing perception, attitude and behaviour. Included in this section is a consideration of the influence of culture on receiver behaviour. Chapter 3 then examines how communication has evolved since the early twentieth century and in particular discusses the influence of propaganda and persuasion techniques, developmental communication, and relational or dialogical communication. The chapter concludes with an introduction to risk and crisis communication theory.

Chapter 4 introduces the concept of strategic communication management. In particular, emphasis is placed on the differences between strategic and tactical communication management. Using a seminal model offered by James Grunig, each of the critical elements of strategic communication management is discussed in turn. The chapter builds on the previous two chapters to address in some detail the practical relationship between strategy, strategic management and communication. It concludes with an introduction to the core subject of this dissertation, strategic major emergency communication management and introduces the reader to a number of models of strategic major emergency communication. This serves as an introduction for a more detailed analysis of the subject matter throughout Chapters 5, 6, 7 and 8 .

Chapter 5 sets out a broad best-practice conceptual framework of strategic communication management during major emergency situations centred on six key strategic communication principles. At the outset, the conceptual strategic framework is introduced in diagrammatic format. Each of the six principles is then discussed in turn and involves a discussion of major emergency communication in the context of the culture and structure of the organisation, ongoing and meaningful dialogue, adopting a scientific approach towards communication, employing stragegic communication processes, integrating all elements of communication, and building strong relationships and trust with stakeholders and the public.

Chapters 6, 7 and 8 describe the series of strategic-tactical steps of major emergency communication management and how they are guided and underpinned by the six strategic principles discussed in the conceptual framework. 
Chapter 6 details the important operational steps that should be taken by the MECIT in order to prepare and plan all aspects of the communication function prior to the occurrence of a major emergency situation - the pre-crisis stage. In this regard communication is considered at two distinct levels, a higher-level and a tactical-level. The higher-level pre-crisis communication strategy steps describe a series of decisions and actions that senior management in the lead response organisation need to consider in order for the tactical-level major emergency communication process to get underway. This includes selecting the senior major emergency communication manager, selecting the members of the MECIT and setting preliminary communication goals. Following this, the series of tactical-level communication steps are discussed, focusing specifically on the key functions of communication prevention and preparation.

Chapter 7 describes a series of strategic-tactical steps that the major emergency communication and information team (MECIT) undertakes when managing all aspects of an effective and compassionate communication response once a major emergency actualises - the crisis stage. Throughout the chapter, each of these steps is dealt with individually. This involves examining the communication function in the context of early identification of major emergency situations, recognising that a major emergency situation has actually occurred, the communication response actions that the MECIT needs to take, and how the MECIT should communicate with members of the public.

Chapter 8 undertakes an analysis of the important communication functions in the post-crisis stage of a major emergency situation. The chapter begins with an examination of communication with stakeholders and affected communities in the aftermath of a major emergency situation. Following this, the thesis examines briefly the role of communication in business continuity management. Chapter eight then examines how communication needs to be evaluated and lessons learned in order to improve the overall process. The chapter finishes with a discussion of how the communication function, during the post-crisis stage, does not end at that point but links immediately to the pre-crisis stage of the next major emergency situation. 
Chapter 9 is an assessment and comparison of how three leading countries (the US, the UK and Sweden) approach their respective strategic major emergency communication efforts. As already stated, this is based partly on original field research undertaken especially for this dissertation.

Chapter 10 considers how the Irish government manages its strategic communication function by examining a broad range of official literature, newspaper reportage and guidance documents. Additionally, using original field research, this chapter provides a detailed account of the attitudes and views of key emergency planners in Ireland to strategic communication management.

Chapter 11 draws conclusions and provides recommendations that may be of value to major emergency communication theorists and practitioners, especially in Ireland. 


\section{Theory of Major EMERgency Management}

\subsection{Introduction}

Despite advances in risk management, many major emergencies occur without warning and their outcomes can be utterly destructive to society. The ability to deal with these events successfully is an extremely important management skill that can save lives and prevent much suffering. Up to the early 1990s, there was a limited amount of literature that dealt with this important subject. A number of significant, high profile disasters resulted in a comprehensive reappraisal of how major emergencies should be planned for and handled. Consequently, a whole field of theory has evolved, centred on major emergency management, together with academic courses, journals, web sites and textbooks related to the subject. The United States, the United Kingdom and Sweden are among the countries leading research and development in this expanding field.

However, notwithstanding great advances in major emergency management and the significant resources allocated to the discipline, countries still struggle to provide an adequate response to major emergency situations. For example, the US government was criticised heavily for its response to Hurricane Katrina in 2005 in which 1,000 people died and 200,000 houses were damaged beyond repair (Padgett 2005: 18). This is despite the US Federal Emergency Management Agency (FEMA) having 2,600 employees and an annual budget of \$5bn (FEMA 2008(a)). Following this major emergency, the US Government undertook a thorough review of its preparedness and response capabilities. In a hard-hitting report (Townsend 2006), many of FEMA's structures and protocols changed. Additionally, many of the major emergency response processes changed, such as the flows of communication within the Joint Information Centre (JIC) (NRT 2000: 2-1). This fact highlights the complex nature of, and inherent difficulties associated with, the management of major emergencies. It also highlights how major emergency practice evolves over time. As mentioned earlier, and as will be seen throughout this chapter, the methodologies 
adopted by countries for major emergency management have changed over the past fifty years. Consequently, best-international-practice in this field of endeavour can be considered only as a temporary quantum, which is likely to evolve over time.

\subsection{Overview of major emergencies}

In order to analyse comprehensively the theory of major emergency management, it is important to have an appreciation of what constitutes a major emergency.

\subsubsection{The social construction of major emergencies}

The occurrence of a major emergency situation is fundamentally dependent on prevailing economic and social conditions, where vulnerable societies and environments need to be exposed to a major hazard. As mentioned at 1.5.1 above, Coppola (2007: 2) usefully defines a hazard as, 'A source of danger or an extreme event that has the potential to affect people, property and the natural environment in a given location.'

Therefore, if human or environmental systems are not actually, nor perceived to be, threatened, a major emergency situation does not exist. For example, McEntire (2005: 207) emphasises, 'a tornado touching down in uninhabited plains is not a disaster'. Salter believes that the impacts of major emergency situations are social products, or 'manifestations of vulnerable communities' (cited in Ingleton 1999: 112). In this regard, vulnerability relates to the degree of susceptibility and resilience of the community and environment to resist major disruption or loss (Smith 1992: 22). However, Salter emphasises:

Vulnerability is differential - we are not all equally vulnerable. Within any group some are more vulnerable than others... to specific hazards (Salter, cited in Ingleton, 1999: 112).

Mileti (1999: 3) concurs with this sentiment stating:

Many disaster losses - rather than stemming from unexpected events are the predictable result of interactions among three major systems: the physical environment; the social and demographic characteristics of the 
communities that experience them; and the buildings, roads, bridges, and other components of the constructed environment.

Therefore, the interaction of people with their environment can attenuate, but in certain circumstances amplify the risk of major emergencies occurring within communities (Royal Society 1992: 114; Beck 1992: 14). For example, developments within the floodplains surrounding New Orleans have been cited as a contributory factor towards the 2005 floods in that region (Mansnerus 2006: 12). Changing demographic patterns arising from social and economic necessity generate further vulnerability within communities. For example, of the 17,000 people who died during the massive 2001 earthquake in Gujarat, India, many were social migrants living in sub-standard housing (Shaw and Sinha 2003: 39). Beck (1992: 20) describes how the social production of wealth by governments and industry is systematically 'unleashing destructive forces' on society. As regards preparation and planning for major emergencies, one needs to consider people's beliefs, attitudes, judgements and feelings in relation to significant risk and hazards as well as considering the physical manifestation of major emergency events.

\subsubsection{Categorisation of major emergencies}

Many theorists have attempted to categorise major emergencies. However, this has proven extremely difficult due to the amorphous nature of these destructive events and the varied and unpredictable contexts in which they occur.

The aetiology of major emergencies identifies a number of physical hazards that are often used in the categorisation of such events. These include natural hazards such as earthquakes, hurricanes, tsunami and insect infestation; technological or anthropogenic hazards such as leaks from nuclear power plants and damaged levees; and social classes of hazards that are associated with terrorist and crowd related major emergencies (Hewitt 1997: 101).

Toft and Reynolds (2004: 14) consider that anthropogenic hazards, such as those described above, should be termed 'socio-technical', as many major emergencies occur due to breakdowns in 'systems' that are often the result of human interaction. Hazards that occur due to the interaction of natural and socio-technical processes can 
be termed 'hybrid' disasters (Shaluf, Ahmadun and Said 2003: 25). An example of a 'hybrid' disaster is the widespread flooding that occurred in New Orleans following the disinclination of the US Administration to provide funding to repair the levees in the region, prior to Hurricane Katrina making landfall (Sandman 2005: 4).

Major emergencies are often considered across a number of key dimensions, thereby allowing them to be categorised from a range of different perspectives. The 'impact' dimension allows major emergencies to be categorised in terms of their 'direct' and 'indirect' consequences on people, the community, the environment and the economy (Trim 2004: 219). The 'scale' or 'magnitude' of a disastrous event provides another useful method of categorisation, which can be measured in terms of deaths, injuries, property damaged, or economic cost (Boin and Lagadec 2000: 186). Closely associated with 'scale' is the 'geographical impact' of a disastrous event, which relates to the physical size of the impact area (Rubin, cited in Ingleton, 1999: 81). The 'predictability' of a major emergency is another key consideration (Hodgkinson and Stewart 1991: 36). Consequently, major emergencies can be categorised as: 'sudden', such as earthquakes; 'creeping', such as landslides; 'foreseeable', such as melting icecaps; 'deliberate', such as war; or 'accidental', including technological disasters (Benthall 1993: 12).

\subsubsection{Major emergency life cycles}

According to Coombs (1999: 9), major emergencies have 'an identifiable life cycle'. This is a consistent theme 'that permeates' contemporary emergency management theory. Recognising the major emergency in terms of a life cycle allows management to sub-divide the entire process into distinct stages. This allows the lead response organisation to comprehend the patterns that exist within a major emergency situation, the key elements involved in each distinct stage and an understanding of how each stage fits within the entire major emergency management framework, thereby ensuring a more effective response.

Theorists have identified a number of life cycle models, including three-, four- and five-stage variants. Managing major emergencies in individual stages is often referred to as 'a systems approach' to major emergency management; a paradigm that has 
been adopted by many leading countries including the USA, Australia, the UK, Canada and Sweden. As a relatively recent phenomonen, the 'systems approach' requires rigorous analysis throughout a range of different categories of major emergency situations before it can be deemed best practice. Richardson (1994: 63) and Coombs (1999: 14) identify three distinct stages in a major emergency situation: a 'pre-crisis stage', involving the critical disciplines of hazard identification, planning, and preparation for an impending disastrous event; the 'crisis' stage that occurs once a significant hazard actualises and involves the speedy initiation of warnings, alarm and response actions; and finally a 'post-crisis' stage that involves the disciplines of relief and recovery efforts, together with strategies to help society to return to normality.

A number of four-stage models exist, such as those adopted by Fink (1986: 20), Coppela (2007: 8) and Alexander (2002: 6). The four-stage approach generally involves the following phases: mitigation, preparation, response and recovery. Adopting a five-stage model, the Centers for Disease Control and Prevention (CDC) describe a major emergency life cycle using the following stages: pre-crisis, initial, maintenance, resolution, and evaluation (Reynolds 2002: 7).

While each model differs in the number of stages involved, intrinsically no significant difference exists between the ranges of functions that need to be conducted to manage a major emergency situation. For the sake of simplicity, the three-stage major emergency life cycle is the model chosen for the remainder of this dissertation.

\subsection{Major emergency management}

Major emergency management is defined as the discipline of applying science, technology, planning and management to deal with extreme events that can injure or kill large numbers of people, do extensive damage to property, and disrupt community life (Drabek and Hoetmer 1991: xvii). Effective major emergency management is predicated on detailed cooperation and integration of plans and resources at all levels of government, public services and non-governmental and voluntary organisations. Many terms have been used to characterise the process of 
major emergency management and include, 'proactive and flexible' (EPS 1998: 7), 'sequential and dynamic' (Heath 1995: 17), 'coherent and integrated' (McEntire 2005: 206), and 'coordinated' (Iwan 1999: 10). Foster, cited in Alexander, (2005: $160)$, warns that 'any inefficiency in major emergency management will be paid for in avoidable casualties and damage'.

\subsubsection{Theory of major emergency management}

Traditional major emergency management theorists placed considerable emphasis on one critical function, the capability of emergency responders to restore normality following the impact of a major emergency. According to O'Brien and Read (2005: 353 ), this approach stems from the 1950s when people were anxious about one category of major emergency, a nuclear attack, which they considered at that time to be inevitable. Recognising shortcomings in this limited approach, Wildavsky (1988) analysed the problem of how to prepare better for major emergency situations. He identified two contrasting strategies, 'anticipation' and 'resilience' (Comfort 1994: 174). Both of these strategies have been used over the past number of years as a basis for developing differing approaches towards major emergency management.

\subsubsection{Anticipation}

According to Wildavsky (1988: 77), anticipation involves a proactive approach to major emergency management by a centralised decision-making function. It also involves a careful assessment of vulnerability in the community in order to limit obvious dangers. He described it as '... a mode of control by a central mind...a coordinated effort...made to predict and prevent potential dangers before damage is done (ibid.)'.

Effective anticipatory measures include predicting hazards and risk assessment (ibid: 78), actions designed to limit the magnitude and frequency of potential hazards (Burton, Kates and White 1993: 113), and improved risk communication (Hood and Jones 1996: 18). However, a number of theorists do not support the precautionary anticipation approach, claiming that the majority of major emergencies can 'be 
anticipated only with the benefit of hindsight' and is suitable only for particular categories of major emergency situations (Royal Society 1992: 156).

\subsubsection{Resilience}

In contrast to the anticipatory approach, Wildavsky (1988: 224) defined resilience as, 'the capacity to cope with unanticipated dangers after they have become manifest...learning to bounce back'. While anticipation focuses on being able to detect and protect against hazards, resilience emphasises taking action against their impact. This can be achieved through processes such as effective emergency warnings, alarms and response; search and rescue; relief and recovery efforts; and ensuring that publics have adequate insurance (Cuny 1983: 53). Supporting the process, Geis (2000: 153) states that resilience 'provides the best means' for developing the most effective disaster and emergency management programmes.

Theorists such as Arjen and Lagadec (2000: 188) explain that the process of resilience is 'an old fashioned way' of dealing with major emergencies. McEntire (2005: 209) states that the approach 'does not capture the social, political, and economic realities of disasters' as there is no great emphasis on meaningful dialogue with publics, thereby allowing a full assessment of their individual needs and concerns.

\subsubsection{Contemporary approaches to major emergency management}

Wildavsky (1988: 186) recognised shortcomings in both the anticipation and resilience approaches. Consequently, he proposed achieving 'a balance between anticipation and resilience' in order to effectively manage major emergencies. This balanced approach spawned the 'integrated emergency management' (IEM) model, which has been used widely in both the US and the UK since the mid-1980s. The IEM model ensures that all response agencies work together to provide a coordinated approach to major emergency situations. The model incorporates critical functions such as major emergency anticipation, assessment, detection, mitigation, prevention, preparation, response and recovery (HM Government 2005(a): 4). While traditional approaches to major emergency management concentrated on single categories of 
hazards, the IEM approach is focused on a broad spectrum of major emergencies categories - termed the all hazards approach (Comfort, Sungu and Johnson 2001: 145; Boin and Lagadec 2000: 188; CCS 2007: 15).

Since the 1990s, the IEM has evolved, or shifted, further to incorporate many holistic elements within major emergency management, including improved levels of communication with, and participation by, 'non-expert' members of the public (McEntire 2005: 210; Von Kotze, cited in Ingleton, 1999: 149). A number of other key changes have been identified in the shift from the traditional practice of major emergency management to the more holistic and contemporary practice. Once again, these shifts in major emergency management are relatively recent developments and as such require rigorous analysis and testing in order to determine their efficacy. While they are considered by many countries to be currently best-internationalpractice, it is likely that these processes will evolve further in order to provide newer and more effective methods of managing major emergency situations. These shifts can be viewed graphically in Figure 2.1 below.

Figure 2.1: Shifts in major emergency management

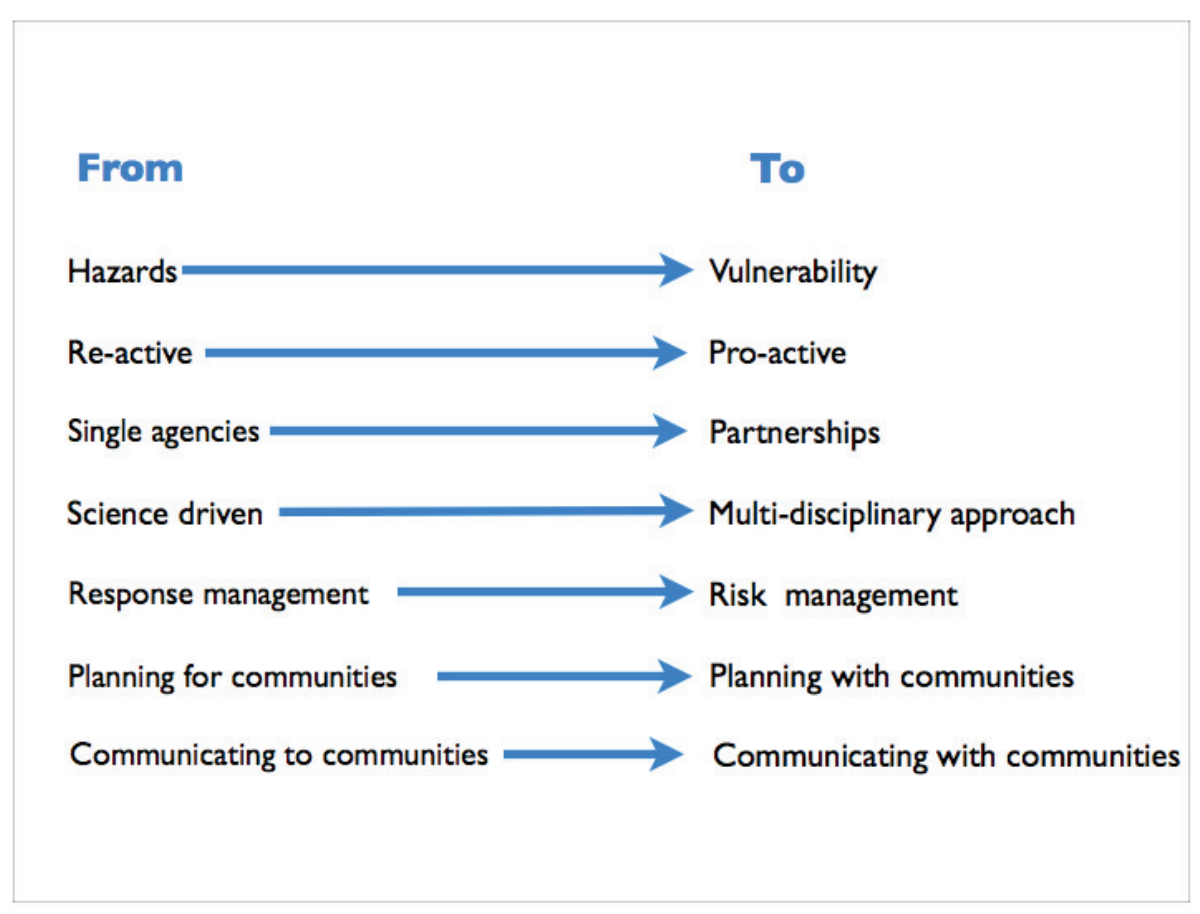

Source: (Salter, cited in Ingleton, 1999: 111) 


\subsection{The major emergency management cycle}

The entire major emergency management process is considered as a series of repetitive events that exist within a comprehensive cyclical model. According to Alexander (2005: 160), major emergency management is a continuous process 'that may become rapidly dysfunctional if allowed to become static'. Consequently, contemporary response organisations adopt a 'systems approach' and carry out specific functions within each distinct stage of the major emergency life cycle. While most major response agencies have their own specific variant of the major emergency management cycle, the key elements are intrinsically similar within a three-, four-, or five-staged approach.

Adopting a model based on work by Alexander (2002: 6) and Coombs (1999: 14), it is possible to outline a composite cyclical model of major emergency management that depicts the key stages of a major emergency situation and the critical functions that need to be considered within each of those stages. This composite major emergency life cycle is presented in Figure 2.2 over. 
Figure 2.2: Composite major emergency life cycle

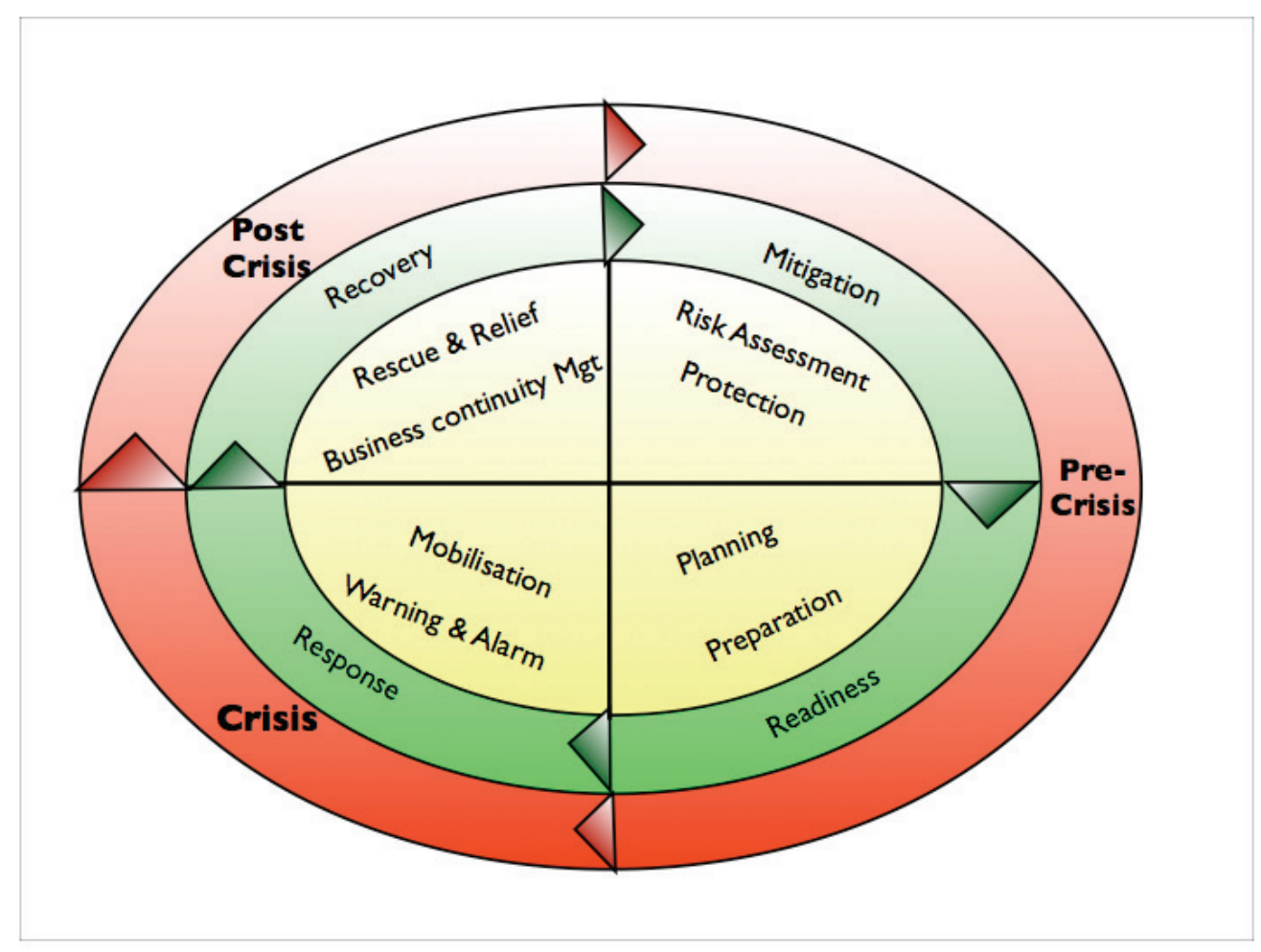

Source: (Based on Alexander 2002: 6 and Coombs 1999: 14)

\subsubsection{Pre-crisis stage}

This first stage of the major emergency management life cycle involves all of the essential functions that must be considered and acted on prior to the occurrence of a serious damaging event. In particular it involves the mitigation and readiness phases (in green) of Figure 2.2.

\subsubsection{Mitigation}

An entire field of science has evolved centred on the subject of mitigation, which involves all long-term efforts to anticipate, assess, and prevent the possibility of a hazard actualising, or to reduce the effects of a subsequent major emergency should prevention measures fail. Mitigation efforts include putting in place structural measures such as building floodgates and seismic retrofitting buildings; and non- 
structural measures such as effective land planning and enhancing community awareness about potential hazards.

The possibility of a major emergency occurring is commonly known as 'risk' and it 'denotes a presumed or possible threat of danger' (Nohrstedt 2000: 137). In order to reduce the risk to life, property and the environment posed by hazards, it is important to engage in an effective risk management process, which is an intrinsic element of the mitigation phase. Risk management can be defined as:

The process whereby decisions are made to accept a known or assessed risk and/or the implementation of actions to reduce the consequences or probability of its occurrence (Royal Society 1992: 5).

Figure 2.3 illustrates a risk management framework and can be useful as a standard process for assisting risk mitigation efforts.

Figure 2.3: Risk management framework

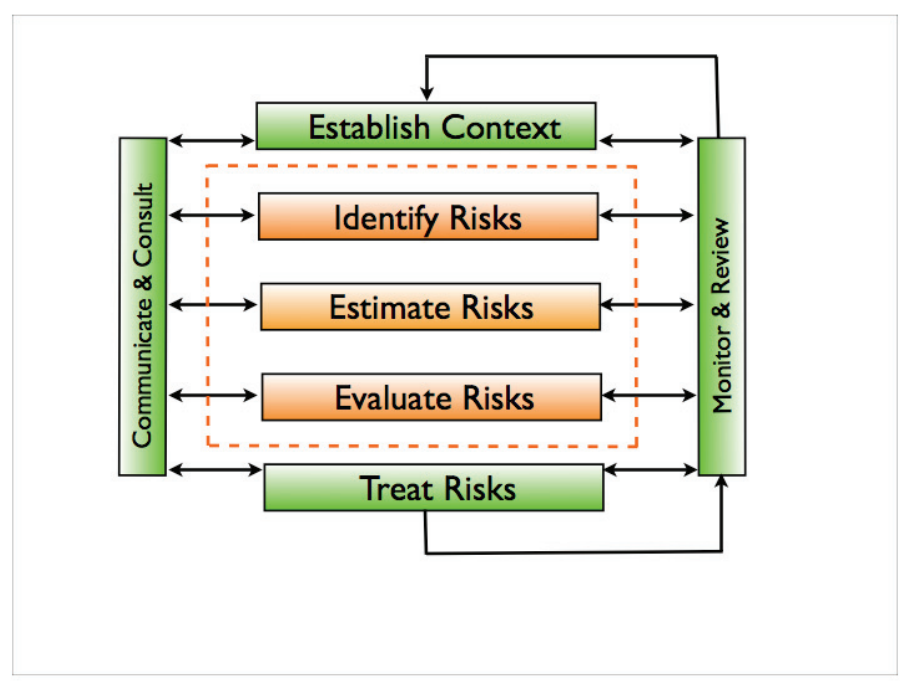

Source: (Shaw and Sinha 2003: 44)

The first stage of the risk management process involves establishing the context, or recording the general characteristics of an area or process, including social political, economic, environmental, and infrastructure elements (Jeynes 2002: 18). Following this, a comprehensive risk assessment process is carried out. Risk assessment is defined as: 
Technical studies that assess the risks and benefits of various risk options and provide information to decision-makers - who then make risk management decisions (Price 1989: 37).

The process consists of three distinct sub-elements (shaded in orange in figure 2.3 before). This first of these sub-elements involves the identification of hazards and risks that are likely to result in major emergencies, utilising tools such as hazard mapping (Alexander 2002: 28), safety audits, hazard surveys, and operability studies (Jeynes 2002: 15). The second sub-element of risk assessment, within the risk management process, involves conducting a comprehensive risk estimate of important conditions such as the potential magnitude of a particular category of major emergency, and its probable impact on populations or ecosystems. Useful tools for risk estimation include 'event' and 'fault-tree' analysis (Royal Society 1992: 17), scenario planning (Ringland 1998: 2), and the use of scales such as the Modified Mercali Scale (Mercali Intensity Scale 2008; Magnusson 1987: 386). The third and final sub-element of the risk assessment process involves the process of risk evaluation. This is defined as:

The establishment of a qualitative or quantitative relationship between risks and benefits, involving the complex process of determining the significance of the identified hazards and estimated risks to those organisms or people concerned with or affected by them (WHO 2008).

A common criticism of the risk assessment process is a tendency for risk managers to engage in an overly quantitative and reductive focus, to the detriment of considering qualitative issues within risk. This can often result in important, non-quantifiable or inaccessible information, such as a person's perception of a hazard, being ignored. In order to conduct an effective risk assessment, it is necessary to understand that different people perceive, and will react to major emergencies, in many different ways, based on scientific information that is available to them and their psychological and cultural circumstances. This is an extremely important factor that needs to be considered when developing major emergency communication strategies and programmes. 
As described earlier, a number of social scientists believe that risk is socially constructed. Consequently, the probability of events occurring in the future cannot be measured simply by conducting scientifically-based quantified assessments (Adams 1995: 10; Toft and Reynolds 2004: 2). Slovic (2000: 5) describes how the process of conducting risk assessment is subjective 'due to a dependence on value judgements at every stage of the process'. Adams (1995: 7) emphasises that risk cannot be considered simply in terms of 'objective' or 'real' risk. Gherardi (1998: 81) concurs and believes that ignoring qualitative factors results in '...a way of not seeing; it is a collective blindness to important issues'. Consequently, contemporary theorists have started to examine the qualitative elements of risk situations, including psychological and psychometric influences (Fischhoff 2004; Covello, Wojtecki and Hyde 2001; and Sjoberg 1998), and cultural factors (Gherardi 1998). Linking cultural and psychometric traditions and research, Wildavsky and Dake (1990: 48) state that '...cultural biases best predict risk perception findings...individuals choose what to fear (and how to fear it), in order to support their way of life'. While Ball (2006: 5) highlights the fact that insufficient weight is sometimes attached to science in the risk decision process, he emphasises that not all theorists dispense with the 'technical component' of risk decision-making. Consequently, this latter category of theorist supports the scientific 'analytic paradigm' (ibid.).

Once the risk estimation and risk evaluation processes have been fully completed, the results are then recorded in a risk assessment matrix. This tool allows particular hazards to be compared and assessed with respect to probability and severity (Alexander 2002: 57). A 'risk characterisation' is also useful and provides decision makers with 'a summary or translation of the results of the technical analysis for use by the decision maker' (Stern and Fineberg 1996: 1).

The final stage in the risk management process, as described in Figure 2.3, involves treating risks. Alexander (2002: 59) states that this final stage is the 'applied side of risk assessment' and involves making decisions and taking action based on the results of that assessment. The Royal Society (1992: 5) defines this stage as: 
The process whereby decisions are made to accept a known or assessed risk and/or the implementation of actions to reduce the consequences or probability of occurrence.

Decisions can be reached using a number of methods, both scientific and nonscientific. The use of tools such as the risk assessment matrix, multi-criterion decision analysis (Royal Academy of Engineers 2002: 11), and cost-benefit analysis (Toft and Reynolds 2004: 8) can be of assistance. Contemporary approaches to risk management emphasise the involvement of a broad range of participants in the risk decision-making process. Meaningfully engaging a broad representative sample of the public in the decision-making process provides a greater depth of knowledge to the process, can assist in reaching better quality decisions, and lessens the chance that the decision will be ultimately rejected (Price 1989: 152). It also engenders public trust and credibility in the responsible agency (ibid: 151). The public engagement process is an important subject within this dissertation, and is discussed in detail later in this dissertation.

While a risk-based society cannot expect to prevent all emergencies or serious accidents from occurring, once decisions have been discussed and agreed, mitigation measures can be put in place in an attempt to treat the hazard and prevent it actualising. This can involve actions such as vaccinating against H5N1 Avian Flu virus (Walsh 2005: 26), developing tsunami warning systems (McWilliams 2004: 10), and managing floodplains (Mileti 1999: 7). As can be seen in Figure 2.3, supporting the entire risk management process are the critical ongoing functions of risk communication, consultation, monitoring and review. Throughout Chapters 5,6,7 and 8 , it will be demonstrated that each of these functions is an essential element of the strategic major emergency communication management process, which in turn is a critical factor in the success or failure of the entire major emergency effort.

\subsubsection{Readiness stage}

In the event that mitigation efforts fail, and a major emergency is imminent, readiness and preparedness of major emergency plans, equipment, personnel and procedures in order to effectively respond to a disastrous event are essential to prevent injury, loss 
of life and damage to the environment. Key elements in this stage include major emergency planning and preparedness at both individual and societal levels.

\subsection{Major emergency planning}

The purpose of major emergency planning is to provide protocols and procedures in order to 'inform, instruct and direct participants' once a situation occurs (Alexander 2002: 99). HSE UK (1999: 1) highlights that comprehensive plans should, 'address all aspects of the response required during every phase of the major emergency, both the immediate needs and the longer-term recovery'. Planning precludes response agencies having to consider routine tasks once a major emergency occurs and facilitates a systematic and effective reaction, thereby helping to contain an incident, prevent loss of life and limit damage (Scarman Centre 2004: 8-16). One of the principal criticisms levelled at major emergency planning is its inability to deal with a broad range of different categories of potential major emergencies. However, many theorists emphasise the importance of developing a generic plan that can be adapted to meet the needs of all types of disastrous events (Coombs 1999: 62). As already described, this approach to planning incorporates the 'all hazards' approach to major emergency planning (EMA 2003: 6; Ridge 2004(b): 2). As Hodgkinson and Stewart (1991: 70) write:

Clearly, it may not be possible to plan for every disaster scenario. However, the real meaning of preparedness is not about being ready for every possible scenario but to develop core preparedness for different emergencies.

Consequently, it is advisable to develop and maintain both generic and high-risk plans (HM Government 2005(a): 50). These plans should deal with all of the distinct stages of the emergency life cycle, including plans for 'scaling-up' the major emergency (ibid.). Included within the planning process should be the development of plans to deal with the secondary impacts of major emergency situations. Examples include public order emergencies, involving widespread rioting and shooting, as occurred in New Orleans following Hurricane Katrina (Gold 2005: 10). An essential component of a major emergency plan is a comprehensive communication plan. 
Major emergency communication planning is comprehensively analysed below in Chapter 5.

According to London Resilience (2005: 17), major emergency plans should be multiagency based, fully recognising the input of the principal response agencies, various professional organisations such as medical practitioners, voluntary groups and the private sector. However, this requires a mutually agreed strategy, clear protocols and procedures for working together, and considerable planning and coordination in relation to information-gathering, sharing and dissemination. Additionally, some theorists emphasise how roles and responsibilities should also be clearly defined within the plan (Riley and Meadows 1997: 354; London Resilience 2005: 14). CCS (2007: 5) emphasises how senior management, at the highest level, should fully endorse and support the planning effort.

According to HSE UK (1999: 10), it is essential that major emergency plans are viewed as 'living documents' and should be considered a process rather than a static goal to be achieved. This requires that the plan be continuously tested and revised (Alexander 2002: 126). All potential responders should be fully conversant with the contents of the plan and this should be practiced continuously and progressively, as plans should be allowed to grow and adapt to changing circumstances (ibid: 95). Jeynes (2002: 93) notes that, 'it is also important that adequate resources and financial provisions be in place to support the major emergency plan'.

\subsection{Major emergency preparedness}

In addition to adequate major emergency planning, in order to ensure a requisite level of major emergency readiness, all individuals and organisations need to be appropriately prepared. This requires that they have 'a clear understanding of their roles and responsibilities and how they fit into the wider picture' (HM Government 2005(b): 7; Payne 1999: 112). Central to major emergency preparedness are the functions of selecting suitable response and recovery staff, and providing them with appropriate and realistic training and exercises for dealing with a range of difficult situations (CCS 2007: 5). Front line agencies obliged to deal with the impact of 9/11 
claim that their training limited their response capability as it 'did not prepare them for managing situations outside their particular skill set' (EMA 2003: 9).

Consequently, training must be realistic and 'scenario based', providing responders with the necessary skills to deal with a range of diverse and escalating situations (ibid: 10).

Once intensive training has been completed, major emergency exercises should be conducted in order to allow the lead response organisation to assess the success of the training programmes and also to validate all major emergency plans. At the most fundamental level, response and recovery personnel can engage in 'paper-feed' and 'table-top' exercises. These can then be augmented by 'simulation' exercises where important aspects of crises can be simulated in order to represent reality and where personnel can act out their response roles accordingly. However, full-scale 'live' exercises provide the best means of comprehensively training and exercising responders (HSE UK 1999: 35). All major emergency exercises can either be 'onsite' or 'off-site' and can be conducted at an individual level or combined (Payne 1999: 114). A lack of integrated realistic exercises was cited as a principal reason for the inadequate response to the Kobe earthquake in 1995 (Heath 1995: 23).

A number of other functions are of significant importance during the major emergency preparedness stage, including, amongst many others: developing and implementing adequate public alert and warning systems and conducting public disaster drills, which can be considered a matter of 'life and death' in the first hours of a major emergency (Morge 2000: 3); providing public education and awareness campaigns in order to enhance knowledge and to generate higher levels of interest (Quarantelli 1984: 5); convening meetings with interested and affected parties for the purpose of sharing major emergency information and reaching consensus; and providing the media with a comprehensive knowledge and understanding of issues related to major emergencies (Ridge 2004(b): 4).

Emphasising the shifts in major emergency management, as described earlier in Salter's (Figure 2.1) model, response agencies in many countries are working much closer with communities in order to provide them with the necessary skills and resources to protect themselves in the event of a major emergency situation. This 
includes providing printed materials such as leaflets and brochures, together with videos and posters. Additionally, in some countries, wider communication and educational programmes are becoming more evident. For example, in Australia, EMA is becoming more involved with communities at risk from bushfires. In this regard they are helping these communities to prepare strategies and plans in order 'that they are better prepared and understand their own responsibilities' should their community be faced with a catastrophic bushfire (Rohrmann 2000: 14; Salter 1996: 9). The Australian government recognises that within an environment of limited resources, particularly in remote indigenous areas, young people capable of assisting their communities in times of major emergency need to be trained as part of a volunteer emergency service (Hocke and O'Brien 2003: 62). According to EMA:

In Australia, emphasis is now being placed on the building of community capacity through the development of social and human capital within communities. Collaboration between governments and non-government agencies, communities and individuals to build on the current assets that exist within communities is required (ibid: 65 ).

As will be discussed later in this dissertation, the communication function has an important role to play in assisting programmes such as these.

\subsubsection{Crisis stage}

Should all mitigation and prevention measures fail to thwart a major emergency from occurring, responders begin to focus on the next stage of the major emergency life cycle, the crisis stage. The crisis stage can occur suddenly and without warning, such as the May 2008 earthquake in the Sichuan province of China, which resulted in the deaths of over 50,000 people (Cloonan 2008: 13). Similarly, the crisis stage can be considered a 'rising tide' or 'smouldering' crisis (Millar and Smith 2002: 3) such as an influenza pandemic, about which experts state 'it is almost inevitable that another influenza pandemic will occur' (Pandemic Influenza Expert Group (Ireland) 2007: 1). Once a major emergency actualises, the priority for all concerned is to ensure basic safety and survival (Reynolds 2002: 26), to contain the event, and to protect critical infrastructure (Ridge 2004(b): 43). The essential elements of a major emergency response 
include warning and alarming the public, and mobilising necessary resources in order to deal with the situation.

The first stage of the response process involves recognising that a major emergency has occurred (Coppela 2007: 254). Once this is complete the next step involves 'declaring' the major emergency. This process may include simultaneous issues of alerts, warnings and instructing information, which may include evacuation orders (Morge 2000: 5). Throughout this process the need for timely prediction is important. Close liaison with the scientific community provides a useful method of hazard forecasting and prediction. Following this stage, the lead response organisation will have to mobilise all necessary resources required to deal with the major emergency. This should be supported by an adequate system of command, control and coordination in order to optimise the utilisation of these resources (Ridge 2004(b): 17; HM Government 2005(b): 21). The core of the external response to a life threatening major emergency is generally provided by the principal response agencies, including organisations such as the fire, police, and health/ambulance services, the coast guard, and local authorities. Key support teams are generally required to assist the principal response agencies in many diverse areas, including incident response, evacuation, specialist interests, media affairs, and casualties (Scarman Centre 2004: 8-23). In order to manage the response effectively, these services will establish 'on-site' and 'off-site' control centres from where they will implement their respective major emergency plans (HSE UK 1999: 13-22). The primary objectives during the response phase are to save and protect life; establish control (thereby containing the emergency); relieve suffering through search and rescue and effective casualty management; maintain critical services and restore normality as soon as possible; protect property and safeguard the environment; and finally to facilitate possible investigations and inquiries (CCS 2007: 17).

A number of guiding principles have been identified for responding to major emergencies, including the need for adequate preparedness, especially in terms of planning and ensuring clarity of roles and responsibilities through a comprehensive 
major emergency plan (London Resilience 2005: 14); principal response agencies should respond to major emergencies through a continuation of their 'normal' day-today roles, albeit at a greater tempo and on a larger scale; all response agencies should exercise effective coordination between, and within themselves and affiliate organisations (Hodgkinson and Stewart 1991: 88); and good communications and telecommunications should be maintained at all times, which are 'at the heart of any effective response' (HM Government 2005(b): 31).

An extremely important guiding principle of major emergency response involves ensuring the effective sharing of information and establishing two-way communication between all responding agencies and across multiple jurisdictions (Toft and Reynolds 2004: 84; Ridge 2004: 6). A major criticism levelled at the response effort during the tsunami in South East Asia, in 2004, was the lack of information sharing between agencies. The reason cited was '...intense rivalry between agencies, competing to spend unprecedented budgets, which did not encourage information sharing' (Walter 2005: 98).

Effective information management systems and a two-way exchange of communication between all interested and affected parties will greatly enhance an effective decision-making process (Price 1989: 151).

\subsubsection{Post-crisis stage}

The principal function in the post-crisis stage is recovery management (or relief management). This is the process of restoring and rebuilding the community in the aftermath of a major emergency and involves critical functions such as rescue, relief and business continuity management. The CCS (2007: 6) notes:

Recovery management encompasses the physical, social, psychological, political and financial consequences of an emergency...the need to plan, manage and undertake those activities that will provide as rapid a return to normality as soon as possible - for both the community and responders.

Many theorists propose that the recovery stage starts within 48-hours of the end of the response stage (ibid.). Others propose that the response and recovery stages 'do not occur sequentially' and that recovery should be part of the combined response effort 
and planned accordingly (HM Government 2005(b): 4). Nevertheless, it is important that there is a seamless, structured transition from response to recovery.

The period of initial recovery is generally characterised by a series of needs that require immediate attention. This involves, according to Shaw and Sinha (2003: 40), 'focusing on the provision of basic necessities to victims of the major emergency and restoring social equilibrium'. As the recovery effort involves a large number of relief agencies, both governmental and non-governmental, a comprehensive coordinated plan is essential in order to avoid duplication of effort or leaving gaps in the relief role. Following the tsunami in Banda Aceh in 2004, Alan Vernon of the United Nations Office of the High Commissioner for Refugees stated:

We have four different structures: the military airlift operation, the government's own big efforts, the community's efforts and the agencies' work. The problem is they all run parallel to each other. There is simply no structure to bring them together (cited in Walter 2005: 90).

Consequently, the foundation for any relief and recovery programme should be developed prior to the disastrous event occurring and placed within the overall major emergency plan. Alexander (2002: 269) believes that this will '...improve the efficiency of the recovery process and reduce suffering in the aftermath of the disaster when conditions will be chaotic and time will be at a premium and demands will be relentless'.

The recovery phase requires considerable effort from many organisations. However, it is likely that the local authority will take the lead role in the rehabilitation and reconstruction of the community. This may involve many diverse tasks such as: the provision of adequate supplies and service support, including food, water, shelter and medical supplies (Shaw and Sinha 2003: 42); managing resources (Home Office 2008: 2); the restoration of basic facilities and services, including electricity, water and telephones (Alexander 2002: 273); preventing communicable diseases (Waring and Brown 2005: 41); assisting in the physical and emotional recovery of victims (Hodgkinson and Stewart 1991: 105); supporting the families and friends of victims through the provision of information, assistance and counselling (EPS 1998: 23); cleaning up damaged areas and landfill sites (London Resilience 2005: 1-6); the 
planning of, and reconstruction of housing and basic infrastructure (ibid.: 276); returning evacuees to their homes following contamination or danger (Porfiriev 1999: 77); managing the financial implications and supporting the long-term recovery of affected communities, including their economic development (Walter 2005: 111); disbursing public donations (Home Office 2008: 9); implementing programmes to mitigate vulnerability to future events (Ridge 2004(a): 5); analysing legal considerations and implementing public inquiries (Home Office 2008: 21); ensuring that 'equity' exists in the response effort (Coppela 2007: 325); and, significantly, restoring trust and credibility in the responsible agency (Fournier 2005: 10). This final function was extremely important for the US Administration in the aftermath of Hurricane Katrina when its reputation as an effective organisation was seriously damaged (Bertrand and Lajtha 2002: 188).

A central element of the post-crisis stage is the implementation of a business continuity plan. This is a validated and rehearsed logistical plan that allows certain essential businesses to recover and restore, either partially or wholly, critical functions that are necessary to supply the community. This includes essential businesses such as producers and suppliers of electricity, gas, food, water and prescription drugs.

\section{In summary}

Chapter 2 has described how major emergencies are categorised and socially constructed. The chapter also highlighted how major emergency management evolved considerably over the past four decades. It was shown how this shift results in a more pro-active than reactive approach from major emergency managers, with a greater focus on risk management and mitigation rather than ensuring a mere response capability. As part of this evolution, response agencies concentrate less on planning and communicating for communities, and provide increased focus on communicating with communities through engagement and dialogue. Consequently, the role of the communication practitioner is becoming more important in this regard. Significantly, major emergencies are now viewed as identifiable life cycles, and major emergency management is seen as a continuous process requiring specific functions to be conducted within each distinct stage. Similarly, communication, in support of the major emergency management function, needs to be considered as an 
ongoing life cycle. The importance and evolution of communication management will be discussed in the next chapter. 


\section{THEORY OF COMMUNICATION}

\subsection{Introduction}

The theory of communication can be confusing due to the existence of a multitude of inter-related and often overlapping concepts and definitions. A principal reason cited for this confusion is that communication encompasses a very wide-ranging field of study (Price 1996: 1). 'Communication' comes from the Latin word communicare, meaning 'to make common' or 'to share' (Pearson et al. 2006: 9). In its simplest format, Redmond (2000: 4) defines communication as 'acting on information.' Theodorson and Theodorson (1969: 83) view communication as 'the transmission of information, ideas, attitudes, or emotion from one person or group to another (or others) primarily through symbols'. Watzlawick, Beavin-Bavelas and Jackson (1967: 49) note, 'no matter how one may try, one cannot not communicate.'

\subsection{Categories of communication}

To comprehend such a broad-ranging subject as communication theory, scholars have categorised the subject in a number of different ways. One of the most common categorisations involves a 'traditional bifurcation of communication', whereby the subject is subdivided into 'mass (public) communication and human (speech) communication' (Salwen and Stacks 1996: xii). While many theorists regard 'mass communication' as an intrinsic form of 'human communication', the subdivision is considered useful for the purpose of analysis (ibid.). Mass communication occurs when a small number of people, in particular the mass media, send messages to a large, anonymous, and usually heterogeneous, audience through the use of specialised communication media (Stiff 1994: 230).

Human communication, on the other hand, is described as 'a special form of communication that occurs between and among people' (Redmond 2000: 6). Human communication can be 'actional', where humans communicate either intentionally or 
unintentionally using a one-way process, and react to one another without a reciprocal effect (Scott and Powers 1978: 43); 'interactional', where participants to the communication process react to the other's actions in sequence (Watzlawick et al. 1967: 129; Mortensen 1997: 93); and 'transactional' where participants involved in communication react to each other simultaneously (De Vito 1997: 28; Johnson-Cartee and Copeland 2004: 52). Based on the scale of participants in the process, human communication can also include 'intra-personal' communication. This involves individuals who engage in the process of thinking, reflection or 'day-dreaming' (DeVito 1997: 6; Williams 1992: 31; Price 1996: 76). Human communication can also involve 'interpersonal communication', which Redmond (2000: 18) describes as '...dyadic communication where two or more people transactionally influence one another, or have a mutual and simultaneous effect on one another'. Finally, it can be classified as 'group communication', which Berger and Chaffee (1987: 339) describe as, 'a set of persons with close long-term ties of association and communication'. These 'groups' can be further sub-classified into groups that are formal, informal, voluntary, involuntary, small or large (Price 1996: 77).

\subsection{Components of communication}

Each of the categories of communication mentioned in Section 3.2 share some generic components. An understanding of these components assists in a greater comprehension of the communication process as a whole, which is turn is essential in order to analyse the subject of strategic major emergency communication management.

Discussing the first of these components of communication, DeVito (1997: 8) states that each category of communication must take place within a 'context'. He identifies three dimensions within which communication can be contextualised, "physical, social psychological, and temporal'(ibid.). These dimensions, 'interact with each other, influence and are influenced by each other' (ibid.). Offering a second key component, McQuail and Windahl (1993: 5) emphasise the importance of a 'source' or 'sender' of information who 'encodes' information prior to transmission. 'Encoding' refers to the translation of purpose, intention, or meaning into symbols 
and codes (Severin and Tankard 1988: 51). A further essential component of communication is a 'receiver'. This is someone who attends to and 'decodes' a message transmitted by a 'sender (O'Sullivan et al. 1994: 283). Receivers 'decode' a message when they attribute meaning to what they have perceived (ibid.). Arquilla and Ronfeldt (1997: 441) describe a fourth generic component, '... signals being transmitted between senders and receivers - that is, about messages and the media through which they get communicated'.

These units of information, or messages, travel through pathways that are known as 'channels' and include interpersonal modes such as sound, touch, light; or mass media channels such as television, radio, internet, and short messaging service (Severin and Tankard 1988: 207). Redmond (2000: 6) describes how the connection made amongst humans through communication, '...involves the use of spoken symbolic language (verbal communication)... as well as non-verbal cues such as gestures, facial expressions, and tone of voice'. Price (1996: 9) describes other ways in which messages are communicated, including written and graphical/symbolic forms. In addition, Cohn (2000: 38) adds visual forms of communication to the mix.

\subsection{Uses of communication}

Theorists have identified many uses for communication. For example, Price (1996: 16) emphasises the 'instrumental' use, where communication content is transmitted in order to achieve or obtain something. Closely related to this is the 'persuasive' function, which refers to 'control' or 'manipulation' of cognitions in order to attain advantage (ibid: 17). Miller, cited in, Berger and Chaffee (1987: 451) identifies the 'social influence' of communication. This is where communication is used to modify behaviour in one of three ways: changing existing responses, reinforcing existing responses, or by shaping new responses. However, according to Tierney, Lindell and Perry (2001: 29) in order to modify behaviour in a desirable way, individuals must hear the information, understand it, and perceive its relevance, a central element of the major emergency communication process throughout all three stages of a major emergency life cycle. 


\subsubsection{Attitudes, beliefs, and values}

Rokeach (1968: 112) defines attitude as 'a relatively enduring organisation of beliefs around an object or situation, predisposing one to respond in some preferential manner'. According to Eagly and Chaiken (1993: 257), 'attitudes are formed and modified as people gain information about attitude objects'. Rokeach (1968: 112) considers that attitudes are 'relatively enduring' and difficult to change. Closely related to attitudes are beliefs. Stiff (1994: 11) defines a belief as 'a single predisposition about an object or a situation'. He also states that, 'an attitude is an organisation of beliefs' (ibid.). A number of theorists, including Walter Lippmann and Harold Lasswell during the earlier part of the twentieth century, examined how communication, through techniques such as propaganda and persuasion, was used to shape public opinion and consequently attitudes. This will be discussed later in this chapter.

A third element of a receiver's predisposition involves values. Grunig (1992: 603) describes a value as a deep-rooted conceptual response about the worth or importance of something. Hofstede (1980: 25) states that values are not rational, 'as they are imbued into individuals very early in their lives'. They involve 'strongly held personal beliefs along with moral and ethical principles' (Susskind and Field 1996: 154). Consequently, "values provide the foundation upon which our attitudes are constructed' (Johnson-Cartee and Copeland 2004: 91).

\subsubsection{Behavioural change}

Behaviour is defined as 'a manifestation of one's fundamental values and consequent attitudes' (Becker and Connor 1979: 37). Much research has been conducted in order to determine the link between the values, beliefs and attitudes of an individual or group, and how they will behave. Johnson-Cartee and Copeland (2004: 92) note, 'the motivation for people's behaviour is an interesting interplay of needs, values, and beliefs'. Redmond (2000: 320) discusses how it is more feasible to change a receiver's beliefs than their values, and how one is even more likely to succeed in changing their attitudes. Goldenbeld, Levelt and Heidstra (2000: 66) identify three broad classes of behaviour, reasoned or planned behaviour, impulsive or emotional 
behaviour, and habitual behaviour. They describe how negative habitual behaviour is the most difficult of the three classes to change (ibid: 76). To achieve long-term desirable behavioural change, communication strategies need to be put in place in order to positively influence not only receivers' attitudes, but also their beliefs and values. In this regard, theorists such as Grunig (1992), Dozier, Grunig and Grunig (1995), Coombs (1999) and Daugherty (2003) emphasise the importance of engaging in a strategic rather than a tactical approach towards communication. It will be seen later in this dissertation how many contemporary major emergency communicators favour a participative or dialogical approach in order to influence positively public attitudes and behaviours. Proponents of this approach believe that dialogue generates an environment where decisions can be taken in an objective way, thereby allowing publics to protect themselves prior to or during a major emergency situation. These include decisions relating to mitigation and protection measures such as retrofitting houses in earthquake areas or preparing personal emergency supplies. It will also be shown that the dialogical approach is a relatively recent development that requires rigorous testing in order to determine whether it constitutes best-practice in relation to major emergency situations.

\subsubsection{Behavioural change and perception}

Theorists have identified a number of factors that determine the strength of the attitude-behaviour relationship and consequently influence the process of behavioural change. This is an extremely important consideration for major emergency risk and crisis communication practitioners who attempt to influence how the public will react and behave in the face of great danger. According to Shaw, Shiwaku and Kobayashi (2004: 41), behaviour is influenced by the perception of the receiver at the time that messages are transmitted.

Cohn (2000: 49) states that a receiver's perception is a good indictor of future behaviour. According to Otway and Pahner, cited in Dowie and Lefrere, (1980: 15), the perception of the receiver's environment is a crucial factor in forming attitudes, beliefs and values, describing how 'people respond to a situation based upon what they perceive it to be'. However, perception of a risk and hazardous situation is difficult to comprehend as it is 'multidimensional' - requiring an individual to be able 
to hear, understand, believe and be able to personalise the dangerous situation (Fischhoff, Slovic and Lichenstein 1982: 241; Tierney, Lindell and Perry 2001: 30). According to Cohn (2000: 33):

Public opinion is formed by perception, not reality. Regardless of what is really happening internally, the way the public perceives a situation becomes the reality.

Accordingly, perception plays an important role in communication as, '...no communicator can assume that people will react to the same message in the same way... and [perception] is influenced by peoples' wants, needs, attitudes and other psychological factors' (Severin and Tankard 1988: 120).

According to Bostrom and Lofstedt (2003: 243), the same risk communication 'can evoke fear in some people and anger in others'. Many theorists highlight how nonscientists often perceive risks in a way that is different from how scientists perceive them (Slovic 2000: 224; Rowe and Wright 2001: 341; Ball 2002: 540). A number of factors affect this diverging perception, including how the receiver perceives the 'source' of a communication. The perceived expertise, trustworthiness, credibility and attractiveness of a message source are relevant (Miller, cited in Berger and Chaffee, 1987: 464). Personal characteristics of the receiver at the time of the communication are also considered important. These include an individual's direct experience or knowledge, values, and vested interest in relation to the subject matter of the communication (Stiff 1994: 58; Slovic 2000: 226). Other personal characteristics include an individual's self-esteem, mood, and frame-of-mind (Severin and Tankard 1988: 184); attitude (Sjoberg 2000: 9), generation/age (Ahearne 2000: 763); their gender (Gustafson 1998: 805), how they cognitively evaluate messages, (such as their selective processes, whereby receivers avoid communication or parts of messages that are not in agreement with their existing attitudes or beliefs) (Roloff, cited in Berger and Chaffe, 1987: 505; Price 1996: 117; Severin and Tankard 1988: 128); insufficient concern (Ball 2002: 535); and, significantly, the social and cultural predispositions of the receiver (Chess, Burger and Hughes-McDermott 2004: 270; Fiske 2004: 26; Sjoberg 2000: 6). 


\subsubsection{Perception, behaviour and cultural theory}

Cultural theorists believe that risk is a social construction that is influenced by people's involvement in their everyday interaction with family, friends and peers. Consequently, an individual's perception of, and likely behaviour in, a hazardous situation should be regarded in terms of the strength of that individual's relationship to social groups and the social structure or nature of such groups. Some cultural theorists claim that, from an anthropological perspective, there are two dimensions by which all cultures can be classified. The first of these is the 'grid' dimension that can be defined as the "total body of rules and constraints that a culture imposes on its people in a particular context' (Mars and Nicod 1983: 124). The second dimension is 'group', which refers to 'the extent to which an individual is coerced by others, through being a member of a bounded face-to-face unit' (ibid:125). Depending on the extent to which a person is oriented to either a 'grid' or 'group' position, four universal predispositions can be identified that describe an individual's perception and likely response to a risk or major emergency situation. These are 'hierarchical', 'individualistic', 'egalitarian' and 'fatalistic' predispositions (Dake 1991: 63). The cultural approach to risk and major emergencies recognises the fact that different people, based on these four dispositions, view dangers in entirely different ways and that there are no simple right or wrong answers to this complex subject.

Additionally, cultural theory proposes that in communicating with different individuals and societies, communication practitioners in order to succeed will have to:

....sensitize themselves to the cultural heterogeneity of their audiences. In the process, they not only will be called upon to tailor their activities to the cultural idiosyncrasies of their publics, but may also have to change certain techniques to suit different cultures (Sriramesh and White, Cited in Grunig 1992: 611).

As a consequence, major emergency strategies and ideas that work well in one country may lose effectiveness when introduced in another (Thompson, Ellis and Wildavsky 1990: 84; Frosdick 1995: 45; Trompenaars 1993: 5). The importance of acknowledging that different nation states are likely to require a different approach to 
major emergency communication management from their respective governments, will be discussed later in the dissertation.

While cultural theory provides a useful insight into understanding how individuals and different societies perceive danger and are likely to behave in the face of lifethreatening events, its approach has been criticised as too reductionist and as oversimplifying the complexities surrounding major emergency situations in the real world.

\subsection{Developments in communication theory}

McCroskey and Richmond, cited in Salwen and Stacks, (1996: 235) emphasise that the study of communication is not a recent phenomenon and assert that 'the oldest essay ever discovered was written about $3,000 \mathrm{BC}$ and consisted of advice on how to speak effectively'. According to Bryant (2004: 390), scholars in Ancient Greece, about the fifth century $\mathrm{BC}$, continued research into spoken communication and are credited with developing the 'rhetorical tradition'. Schramm (1997: 6) describes ancient scholars such as Cicero, Aristotle, Homer and Quintillian as the 'forefathers of the forefathers' of modern communication. Their approach to communication provided a foundation for developments in communication theory and research throughout the twentieth century (Price 1996: 240).

According to Willey and Rice (1933: 216), communication in society during the early part of the twentieth century, developed 'without plan or aim'. Despite this, a number of US theorists were actively studying important elements of communication. Due to the post-war reconstruction efforts in Europe and the lack of funding available in their universities, the communication field 'was largely open for the invasion of American ideas' of communication theory and methods of enquiry at this time (McQuail et al. 2005: 1).

One of the earlier US communication theorists, Charles Cooley (1864-1969), in his text Social Organization: a Study of the Larger Mind (1909: 61), defines communication as '...the mechanism through which human relations exist and 
develop - all the symbols of the mind, together with the means of conveying them through space and preserving them in time'. Cooley in his writing emphasised, at an early stage, the centrality to society of human communication. A little later, Walter Lippmann (1889-1974), following up on Cooley's work, was one of the first theorists to consider communication in terms of human perception and reality - an important element, it will be shown, of major emergency communication. He examined how 'individual opinions can differ from those that are expressed in the outside world' (Lippmann 1922: 3). Lippmann also described the phenomenon of agenda-setting, whereby democracy, in his opinion, is 'controlled' by educated elites (Lippmann 1925: 197). Identifying barriers that exist between the public and an event, Lippmann examined how the media can manipulate events or present limited information to the public in order to shape opinions (Lippmann 1922: 28). In this regard, Lippmann was one of the earliest theorists to examine the formation of public opinion, the concepts of social influence, and in particular the study of persuasion and propaganda (Johnson-Cartee and Copeland 2004: 3; Berger and Chaffee 1987: 457). Studies such as these provided a good foundation for later research into risk and major emergency communication. These will be discussed later in this dissertation. In his studies of democracy and communication, John Dewey (1859-1952) argued that 'communication can alone create a great community'(1927: 142). Dewey's work provided a good foundation for later theorists, analysing social communication that is an important concept in the development of resilient communities. This concept will also be discussed later.

However, it is today generally accepted that the study of communication was established as a science following 'brief forays' into the subject by a number of social scientists in the 1930s and 1940s. This work was undertaken principally through academic institutions such as Columbia University and the University of Chicago (Salwen and Stacks 1996: 400). During this period, in an attempt to develop an empirical foundation for the subject, scholars began to introduce scientific techniques, such as quantitative analysis and the use of surveys, into communication research (Jensen and Jankowski 1991: 49; Czitrom 1982: 122). At the forefront of this communication research, at this time, were a number of notable scholars including Lasswell, Lazarsfeld, Lewin and Hovland, whom Schramm (1997: 6) described as the 'forefathers of contemporary communication study'. As proponents 
of Critical Theory, which originated in the Frankfurt School, these theorists '...borrowed from the intellectual tradition that had been growing up in the hard sciences and the social sciences...they studied human communication....and were keenly aware of the relationship of mass media to the problems they were studying' (ibid). Of particular interest to these theorists, during this time, were the effects of communication in life. In this regard, Lewin focused on communication in groups; Lazarsfeld closely analysed the mass media and its effects on people; Hovland used controlled experiments to study attitude change through broadcast media; while Lasswell afforded considerable attention to content analysis and the effects of propaganda. Each of these areas of research, it will be shown later in this dissertation, provides an important basis for many of the techniques employed by major emergency communication practitioners over the past twenty years.

\subsubsection{Building on the rhetorical tradition-propaganda and persuasion}

Of particular interest in the 1940s and 1950s, principally as a result of the Second World War and the subsequent Cold War, were the fields of propaganda and persuasion; two techniques firmly rooted in the rhetorical tradition of communication. Actively researching both propaganda and persuasive techniques, many behavioural scientists at this time were '...observers of, and active participants in, defining the meaning of the Cold War. They contributed to a portrait of the enemy that both reflected and fuelled ideological strains within the American body politic' (Robin 2001: 15). Throughout this time the US government expanded its support and use of academics including social scientists, psychologists, and communicators in order to promote overtly American interests through propaganda and persuasion techniques (Parry-Giles 1994: 449). During this period, scholars such as Lasswell, Blumer and Waples formed a temporary working group called 'the committee on communication and public opinion'. This committee essentially served as an academic and educational adjunct of the Federal Government and was closely linked to the Office of War Information. The establishment of the committee reflected the increasing interest in communications of the US government. 


\subsubsection{Propaganda}

Propaganda, according to Laswell, cited in Severin and Tankard, (1988: 103) and Johnson-Cartee and Copeland (2004: 7), involves the crafting of messages that are designed to exert influence on group members, thereby shaping their perceptions or impressions. Ellul (1965: 61) defines propaganda as:

A set of methods employed by an organised group that wants to bring about the active or passive participation in its actions of a mass of individuals, psychologically unified through psychological manipulation and incorporated into a system.

While similar in many respects, propaganda differs from persuasion in its purpose. According to Grunig (1992: 288), 'communication by propaganda is manipulation'. While Lippmann (mentioned earlier) was actively writing propaganda, Harold Lasswell (1902-1978) was undertaking an empirical analysis of the field. Lasswell studied the propaganda techniques of different nations during the First World War and sought to determine the most effective methods. Lasswell's study was based on a quantitative content analysis of German school texts at that time, in which he examined the use of terms such as 'national superiority', 'foreign inferiority' and 'military heroes' (Rogers 1994: 213). This study proved of considerable interest to, and gained prominence in, the US military-intellectual complex (Robbins 2001: 56). While many German sociologists and American revisionist historians considered propaganda technique 'a vicious social evil to be detected and combated' (Smith 1994: 219), Lasswell viewed propaganda as 'the study of power in society' (Schramm 1997: 34). In his work, Lasswell analysed how 'isolated individuals are exposed to powerful media institutions engaged in reinforcing or changing social behaviour' (Hardt 1992: 89). He also analysed how propaganda could be utilised to shape public opinion within a democratic system. Propaganda according to Lasswell is 'the new instrument for welding millions into one amalgamated instrument' (Schramm 1997: $35)$.

Lasswell believed propaganda to be 'no more moral or immoral than a pump handle' (Robin 2001: 65). He believed that all governments in an age of mass communication used propaganda techniques in order to 'promote their policies' and that propaganda 
had become 'a natural function of politics' (Smith 1994: 219). Many governments used propaganda techniques to communicate important major emergency messages throughout the 1960s, particularly concerning nuclear safety issues. While these messages contained much useful information, many of the messages contained strong political undertones. Additionally, as the propaganda technique was a one-way process of communication, which did not allow a mechanism for receiver feedback or for organisational learning, its effectiveness as a communication approach was considered limited.

\subsubsection{Persuasion}

Persuasive strategies include 'rational appeals' that are based on logic and reason, and 'emotional appeals' that are based on an individual's beliefs, attitudes, fear, outrage, or sympathy (Stiff 1994: 120; Berlo 1960: 12; Miller and Steinberg 1975: 12). One of the earliest investigations concerning the technique of persuasion was conducted by LaPiere (1934). He concentrated on people's attitudes and subsequent behaviours. Persuasive communication became popular during the Second World War, as the US Army wanted academics to provide them with practical guidance concerning its instructional messages. Consequently, important work by Hovland and his associates identified the short- and long-term attitudinal and behavioural effects on audiences, including the sleeper effect, as a result of viewing army training films. Hovland identified a number of characteristics that affect the persuasion process including source credibility, expertise and trustworthiness (Stiff 1994: 90). However, it took until the 1980s for the first substantive persuasive model to be developed, which was Petty and Cacioppo's (1981) Elaboration Likelihood Model (ELM). Petty and Cacioppo hypothesised that one factor affecting people's willingness to scrutinise the content of a persuasive message is their involvement with the message topic.

Consequently, when message receivers are highly involved with the message topic, such as publics in the immediate path of a major hurricane, message characteristics such as high quality supporting evidence and organisational credibility can greatly influence attitude change (Stiff 1994: 109). Research on persuasion remains extremely germane to major emergency communication today, as will be discussed later. 
A guiding model of social influence that helped to shape the study of propaganda and persuasion was Lazarfield and Katz's 'two-step flow of communication'. The theory divides the population into 'active' and 'passive' participants, or 'opinion leaders' and 'followers' according to their interest and activity in relation to an issue, the media and their messages (Servaes cited in Casmir, Fred L. 1991: 55). The theory examines the process intervening between the media's direct message and the audience's ultimate reaction to that message. It highlights the importance of opinion leadership and personal influence while channelling important media messages to the public. Opinion leaders in the context of major emergencies can include medical practitioners, the police, academics and elected representatives.

\subsubsection{Other contributions of Lasswell, Lazarsfeld and Schramm}

\section{Lasswell}

One of Lasswell's most significant contributions to the work of contemporary major emergency communication was his early identification of three social functions of communication through the news media, government and schools. The first of these Lasswell described as 'surveillance'. It involves the collection of information that society should know about and refers to the needs, threats and opportunities to which a society should respond. Secondly, 'correlation' involves society's response to this information, including its organisational and behavioural response. Finally, Lasswell identified 'transmission', which involves the passing on of knowledge and values to society (Lasswell 1948: 276). Each of these three functions remains pertinent to the development of major emergency communication strategies today.

In addition, Lasswell's research contributed greatly to the overall study of communication. One noteworthy aspect was his 'convenient way to describe an act of communication', involving not only the elements of the communication process such as communicator, message, medium, receiver, and effects, but also integrated evolving fields of communication such as research, media and audience analysis, and effect analysis (Lasswell 1948: 37). 


\section{Lazarsfeld}

Lazarsfeld recognised that 'freedom of speech is now a three-cornered proposition between the government, the communications industry and the individual citizen' (Lazarsfeld and Field 1946: 74). In this way, Lazarsfeld identified that a separation of powers exists between these important entities and recognised the importance of individuals having control of their own political destiny. In this regard, Lazarsfeld helped to lay the seeds for a dialogical approach towards communication that will be discussed later in this Chapter.

\section{Schramm}

However, it is Wilbur Schramm who is considered by many to be 'the true founding father' or 'driving force' of communication studies (Griffin 2003: E-1; WahlJorgensen 2004: 560; Rogers 1994: 1). Reardon and de Pillis, cited in Salwen and Stacks, (1996: 400) describe how Schramm developed communication studies as a 'crossroads discipline' to complement the five established social sciences of psychology, sociology, political science, economics and anthropology. Schramm argued that the mass media has the potential to widen horizons, to focus attention, to raise aspirations, and to create a climate for development. Significantly, he took cultural linkages into account, acknowledged the resistance to change and urged an 'understanding participation' (Kumar cited in White 1994: 82). While Schramm's principal interest in this regard was developmental communication, the principles are equally important for major emergency communication management.

\subsubsection{Developmental communication}

In the aftermath of the Second World War, communication was used in order to promote development and to bring about positive social change, through a 'modernisation paradigm', particularly in 'third-world' countries (Jacobson cited in White 1994: 67). This form of communication theory, known as developmental communication, became recognised as an academic discipline in the 1950s, principally through the work of Schramm and other theorists such as Lerner and Rogers. Developmental theorists believed that the problems of underdevelopment or 'backwardness' could be solved by 'a mechanical application of the economic and political systems of the Western countries' (Servaes et al. 1996: 31). Consequently, 
many of the propaganda and persuasion techniques that dominated the Cold War period were utilised in the early stages of developmental communication. However, these techniques were criticised, particularly by theorists such as Beltan and Dagron in the undeveloped nations. They considered developmental communication to be principally a one-way process where messages simply passed from sender (western governments) to receiver (the poor of developing nations), involving processes of 'explanation and teaching...rather than knowledge sharing' (Jacobson cited in White 1994: 67; Servaes et al. 1996: 33).

\subsubsection{Participatory communication}

Believing developmental communication to be 'outdated', theorists began to look at alternative, more inclusive approaches to communication during the 1960s.

Consequently, a form of communication evolved that became known as 'participatory communication', which centred on two-way, dialogical forms of communication. In this regard, 'what was said became secondary to how it was said and to the way it affected others' (Griffin 2003: 25) and relationships became more important than message content. Servaes, cited in Dervin and Voight, (1989: 225) reinforces this point, stating:

The former hierarchical, bureaucratic, and sender-oriented communication model has been replaced by a more horizontal, participative and receiveroriented approach...based fundamentally on interactive, participatory, and twoway communication on all levels of society.

Amongst the leading exponents of the participatory approach to communication at this time were Jurgen Habermas and Paulo Friere. Friere (1983: 76) incorporated concepts such as the cultural identity of communities and participation at all levels. Habermas proposed communication as a basis for the exercise of legitimate power, which can only occur through 'a process, which is at base a fair or consensual dialog among participants and citizens' (Jacobson cited in White 1994: 70). This horizontal communication approach differed from the developmental communication approach, which was principally top-down by nature. This approach to participatory communication provides the basis for contemporary thinking towards the 
management of major emergency communication, which has evolved from the rhetorical tradition to a more 'relational' approach.

\subsubsection{Move towards relational communication}

Whereas the 'rhetorical approach' focuses on social influence that can be considered 'interactional', the 'relational tradition' examines communication from a 'transactional' viewpoint (Salwen and Stacks 1996: 234). In this approach two or more people or groups coordinate their communication in order to reach a shared perspective that is likely to be satisfactory to all. All sides acknowledge the concerns of the other sides, focus on building long-term relationships, act in a trustworthy manner, and meaningfully involve a broad range of participants, both expert and nonexpert, in the decision-making process.

Many theorists consider 'relational' communication more ethical than the 'rhetorical' approach and have produced theories and models supporting the concept. These include Cutlip, Center and Broom's (2000: 249) 'co-orientation model', Susskind and Field's (1996: 37) ‘mutual gains approach', and Stern and Fineberg's (1996: 160) 'broad-based analytic-deliberative process'. The 'relational' tradition of communication is gaining many supporters in the context of strategic major emergency communication management. This is in keeping with the shift towards working with communities rather than for communities, described by Salter earlier in Chapter 2. This subject will be discussed in detail later in this dissertation.

\subsubsection{1990s -}

In the 1990s, Putnam (2001: 40) believes that communication theory 'lacked cohesion... with a splintering of basic concepts and theories that developed into selfcontained enclaves...offering a period of fragmentation'. Many diverse and pluralist sub-specialities of communication were developed during this time, including within them the consideration of communication in the organisation. In particular, Grunig (1992: 539) describes the efforts that were directed towards 'channels, flows, and networks of communication throughout the organisation... and how communication 
defines the organisation as a whole'. Other specialist fields of communication that developed during this period include research into how race, class, age, sexual orientation and ethnicity influence the communication process (Jones 1999: 149). Communication scholars in the $21^{\text {st }}$ century have focused much of their attention on the issue of the "digital divide...the gap between those who have access to technology and those who do not' (Besser 2004: 1). In particular, theorists are interested in examining how income and education can preclude lower socio-economic groups from having access to important public service communications (Bryant 2004: 394; Besser 2004: 1).

The study and practice of risk and crisis communication is a relatively recent phenomenon, with a growing volume of literature extant since the 1980s (Royal Society 1992: 118; Price 1989: 17). Palenchar and Heath (2002: 129) discuss how 'iconic incidents' such as those at Three Mile Island in 1979 and Bhopal in 1984, resulted in a manifest interest in the subject of risk and emergency communication theory and practice. Much of the effort during the 1980s and 1990s was directed towards preparing communication plans for environmental disasters. However, the so-called 9/11 attacks in the United States in 2001 and the London and Madrid train bombings have focused contemporary risk and emergency communication theorists' attentions on terrorist related incidents.

\subsection{Communication management}

Gamble and Gamble (2005: 7) describe how 'communication is the deliberate or accidental transfer of meaning'. In order for deliberate communication to be effective, it should be managed. Kaye (1994: 12) defines communication management as 'the process of coordinating the interpretations or meanings construed by interacting people'. Therefore, the process involves both 'understanding and negotiating the meanings of two or more individuals' (ibid.). Managing communication is particularly important within organisations. Consequently, Grunig and Hunt (1984: 6) describe communication management as: 
The overall planning, execution, and evaluation of an organisation's communication with both external and internal publics - groups that affect the ability of the organisation to meet its goals.

A school of thought is emerging, including theorists such as Grunig, Dozier and Daugherty that emphasises the importance of managing communication strategically. This will be discussed in greater detail in Chapter 4 .

As was discussed earlier in this chapter, in the true 'relational tradition', Hutton (1999: 200) describes how the new paradigm of communication management involves 'building relationships with publics'. Van Ruler and Verčič (2004: 3) state:

That - in Europe at least - public relations researchers find it difficult to distinguish between communication management and building relationships.

The effectiveness of communication management performance can be measured by identifying positive effects among clearly defined target publics (Grunig 1992: 188), protection of the organisation's reputation (Huang 2004: 337), and the strength of the organisation-public relationship (Susskind and Field 1996: 41).

\subsubsection{Models of communication management}

Most models of communication management focus on the different approaches adopted by practitioners of the science. In one of the earlier models, Thayer (1968: 56) offered two contrasting concepts. The first of these, 'synchronic' communication, describes how practitioners attempt to synchronise the behaviour of the public with that of the organisation, thereby allowing the organisation to carry on its activities in an uninterrupted manner. 'Diachronic' communication, on the other hand, describes attempts to agree a situation that is acceptable to both the organisation and its publics. Using Thayer's work as a basis, James Grunig developed four seminal models of communication management involving two distinct variables, direction and purpose. Grunig (1992: 289) explains these variables as follows:

Direction describes the extent to which the model is one-way or twoway. One-way communication disseminates information; it is a monologue. Two-way communication exchanges information; it is a dialogue. Purpose describes whether the model is asymmetrical or 
symmetrical. Asymmetrical communication is imbalanced; it leaves the organisation as is and tries to change the public. Symmetrical communication is balanced; it adjusts the relationship between the organisation and public.

Culbertson and Chen (1996: 4) provide succinct descriptions of Grunig's four models of communication management. They categorise the first two models as 'one-way' processes of communication. Organisations practicing 'one-way' communication management do not engage in research or any attempt to understand their publics, nor do they engage in any dialogue or attempt to receive audience/publics feedback. Consequently, Grunig, Grunig and Dozier (2002: 64) consider 'one-way' models 'unethical'. They term the first of the 'one-way' models 'press-agent' or 'publicity' models, wherein emphasis is placed on achieving publicity for the organisation, and minimal attention is afforded to ensuring accuracy or truth in communicated messages. The second 'one-way' model, is termed a 'public information' model, and involves the dissemination of positive information about the organisation by 'journalists in residence', through 'controlled media' (Grunig 1992: 18). Once again no research or evaluation is conducted. However, unlike the 'press agent' model, the 'public information' model involves some attempts to disseminate information that is both accurate and truthful.

Grunig's final two models involve 'two-way' communication processes. In the 'twoway asymmetric' model, practitioners conduct extensive research of their key publics. This is done in order to optimise persuasive strategies, with a view to changing public attitudes and behaviour in a way that best suits the organisation (Grunig 1992: 289). This model and the two 'one-way' models mentioned above are associated with 'rhetorical' approaches to communication, already described in Section 3.6.1.

The fourth model, 'two-way symmetric', which is associated with 'relational communication' previously described in Section 3.6.2, involves the organisation entering transactions with publics as equal partners. In an effort to avoid or manage conflict, the communication process should involve, '... argumentation and debate...however dialogue, listening, understanding and relationship must also take place' (Grunig, Grunig and Dozier 2002: 32). This is particularly effective when 
communicators play an active role as advocates of the publics' interests in strategic decision making. Dozier, Grunig and Grunig (1995: 13) believe that:

Two-way symmetrical communication is a way to develop "win-win" solutions for conflicts between organisations and publics... where both involved can win as a result of negotiation.

Grunig, Grunig and Dozier (2002: 11) emphasise that 'organisations get more of what they want when they give up some of what they want'. A number of other theorists advance the 'two-way symmetrical' model as an optimum approach to managing communication, including, amongst many others, Deetz, cited in Griffin, (2003: 288), Cutlip, Center and Broom (2000: 3), Salwen and Stacks (1996: 465), and Heath (2001: 523).

However, not all scholars are in agreement with the views of these theorists, and many consider the 'two-way symmetrical' model as 'unrealistic and overly idealistic' (L’Etang and Pieczka 1996: 154), 'naïve and utopian' (Maloney 1997: 140), 'an attempt to make an inherently evil practice look good' (L'Etang and Pieczka 1996: 105), and a mere 'tacit or latent strategic act rather than a communicative one in the Habermassian sense' (Nohrstedt 2000: 144).

While recognising merits in the 'two-way' symmetrical model, Murphy (1991: 118) suggests that organisations should pursue communication management using a 'mixed-motives' approach. She emphasises the importance of organisations attempting to satisfy their own needs and interests, while simultaneously trying to help publics to satisfy their best interests. Consequently, Murphy proposes a fifth category of communication management model, the 'mixed-motives' model. This is based on a combination of Grunig's 'two-way asymmetrical' and the 'two-way symmetrical' models where the ideal position for both the organisation and the audience/publics is within the 'win-win' zone. The relationship between the 'mixedmotive model and both of the 'two-way' models is explained by Murphy diagrammatically in Fig. 3.1. over. 
Figure 3.1: Mixed-motives model of communication

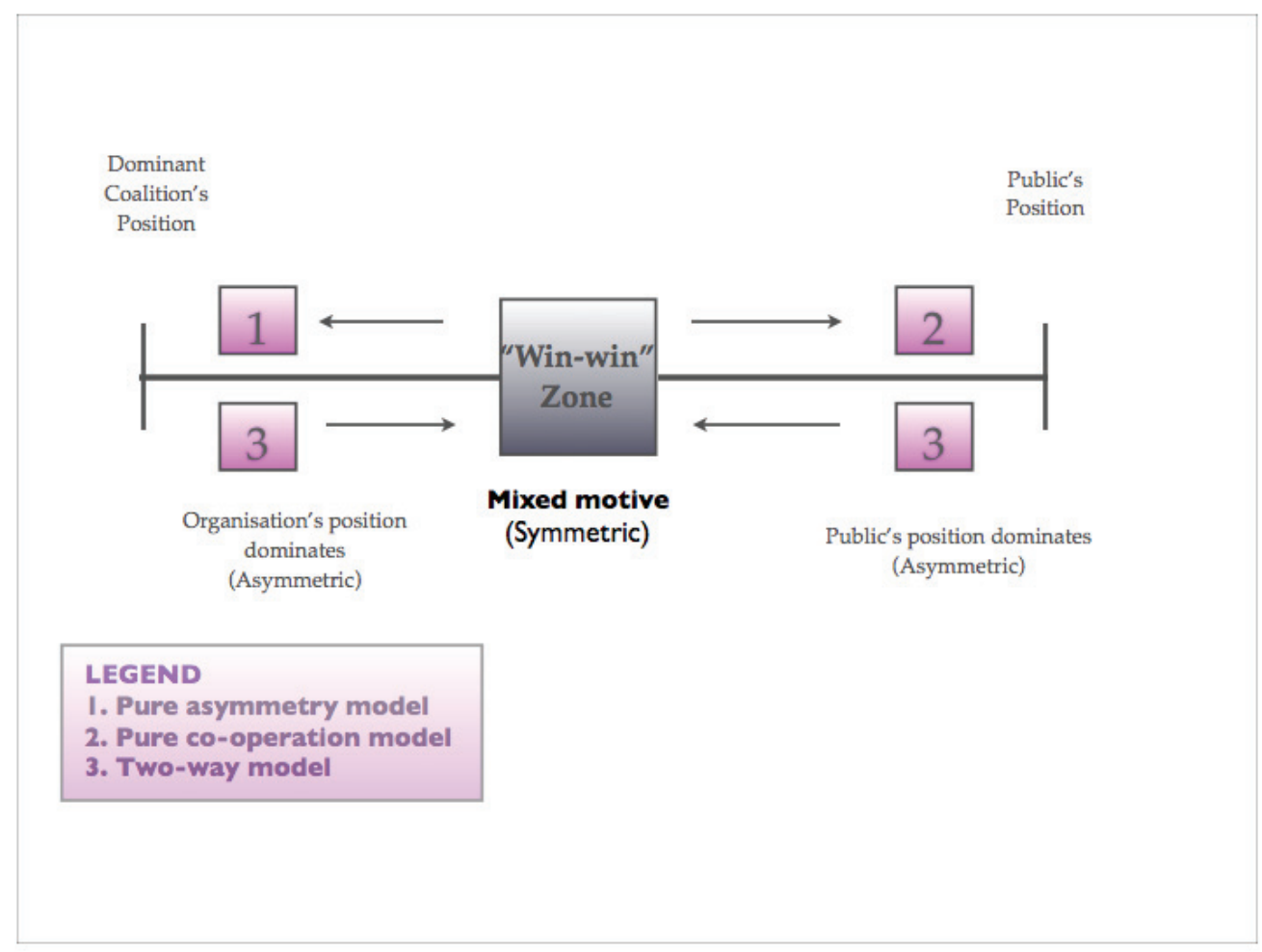

Source: (Murphy 1991: 118)

\subsection{Risk and crisis communication theory}

As discussed in Chapter 1, there exists a somewhat fragmented body of literature in relation to risk and crisis communication theory. This is in no small way due to a limited convergence between scholarly literature, official guidance documents and protocols, and praxis within institutions with responsibility for developing risk and crisis communication policy. Consequently, much of the terminology, such as the meaning of 'risk communication' and 'crisis communication', often becomes interchangeable and confusing. In the previous chapter, Figure 2.2 described a model of a major emergency life cycle and the chapter recommended steps that planners should take in order to prepare for, and to anticipate, life threatening events, thereby allowing for an effective response. A critical factor that is central to the success or failure of effective preparedness, anticipation and response is major emergency 
communication management, which encompasses the functions of risk and crisis communication management.

\subsubsection{Risk communication}

Risk management is a subject that was dealt with in some detail in Chapter 2. The important role that communication plays in risk management was clearly highlighted. Sandman (2002) emphasises that, 'risk communication involves communicating with publics about things that might go wrong'. It can be defined as:

An interactive process of exchange of information and opinion among individuals, groups, and institutions...involving multiple messages about the nature of risk (Price 1989: 21).

Offering a more practical definition, the United States National Academy of Sciences (2002: 1) describes risk communication as the provision of 'timely and credible information to the public in order that appropriate protective actions can be taken'. The US Centers for Disease Control (CDC) (Reynolds 2002: 6) explain how the risk communicator, '.. hopes to provide the receiver with information about the expected type (good or bad), and magnitude (weak or strong), and probability of an outcome, from a behavior or exposure'.

Price (1989: 26) believes risk communication is successful when:

It raises the level of understanding of relevant issues or actions and satisfies those involved that they are adequately informed within the limits of available knowledge.

Many theorists have identified key pre-requisites for successful risk communication, including source credibility (Alesch et al. 2004: 162), using understandable language (Nordlund 1994: 13), demonstrating openness and competence (Darrell 2003: 21); and being consistent in disseminated messages (Reynolds 2002: 11).

As the communication about risk issues takes place prior to the actualisation of a crisis situation, Reynolds (2002: 5) highlight that risk communicators should be under no great time pressures for the development, testing and dissemination of optimum risk messages to publics. 


\subsubsection{Crisis communication}

Crisis communication relates to negative events that are occurring, or have just occurred, often unexpectedly, and the urgent need to communicate about that crisis to stakeholders and the public (Fearn-Banks 1996: 2). This form of communication is undertaken during the 'crisis stage' of a crisis lifecycle, described earlier in Figure 2.2 Sandman (2002) notes that crisis communication 'deals with things that have already gone badly wrong'. It differs from risk communication in that the event is occurring or has already occurred, and publics and the media are awaiting a response. The benefits of effective crisis communication include, the provision of essential information regarding the crisis to stakeholders, assisting the crisis management team with its decision making (Hale, Dulek and Hale 2005: 113), displaying the organisation's authority and capability to deal with the crisis, and utilising the media in order to effect an optimum response and recovery (Fernandez and Merzer 2003: 7).

Due to the 'urgent and unexpected' nature of a crisis, and the lack of control over the timing of the event, Reynolds (2002: 5) emphasises that communication managers will face considerable time pressures, with less time to develop, test and disseminate optimum crisis messages.

\subsubsection{Crisis and emergency risk communication}

This is a hybrid form of communication that is sometimes neglected by theorists and emergency planners. It occurs during the 'crisis' and 'post-crisis' stage of a crisis life cycle and involves the need to communicate information relating to the existence of further risks following a crisis situation. A safety warning relating to the possibility of water contamination following the tsunami in South East Asia in 2004 is a relevant example (Walter 2005: 109). Crisis and emergency risk communication allows publics to make best possible decisions about their well-being following a serious life-threatening event and within very narrow time constraints. 


\subsubsection{Evolution of risk and crisis communication}

Fischhoff describes how risk and crisis communication has evolved through developmental stages or 'focal communication strategies' since the mid-1970s. The stages are as follows:

- All we have to do is get the numbers right

- All we have to do is tell them the numbers.

- All we have to do is explain what we mean by the numbers.

- All we have to do is show them that they've accepted similar risks in the past

- All we have to do is show them that it's a good deal for them.

- All we have to do is treat them nice.

- All we have to do is make them partners.

- All of the above.

Source: (Fischhoff 1995: 138)

The five models of communication management, discussed earlier in Section 3.7.1, closely reflect Fischhoff's eight developmental stages described above. Leiss and Powell (1997: 35-38) summarise these eight stages, and in doing so identify three distinct periods during which risk and crisis communication management has evolved. The first period, which occurred between 1975-84, witnessed considerable emphasis being placed on source-orientation, message content, and in particular, quantitative expressions of risk estimates and scientific validity (Palenchar and Heath 2007: 122). During this time, the public's understanding of issues of science and risk was 'conceived as a deficit... and scientists were presumed to be in possession of the truth' (Dora 2006: 167). Additionally, risk and crisis communication practitioners tended to view with scepticism any research into areas such as risk perception and stakeholder segmentation, and pursued instead strategies based on the 'one-way' model of communication. Lofstedt, Ikeda and Thompson (2000: 157) termed their chosen strategy 'decide-announce-defend'. Communication practitioners, at this time, hoped these strategies would 'do the trick' in terms of communicating with their publics (Fischhoff 1995: 138). Palenchar and Heath (2007: 122) describe how this period was dominated by a 'scientific positivism, whereby data and methodologies of 
scientists dominated how the public assessd risk issues. Dora (2006: 167) terms the paradigm adopted during this period as 'a technocratic approach' within which a bottom-up communication process 'had no place'. It was believed during this period that 'science provided not just a necessary but also a sufficient basis for policy decision-making' (ibid: 168).

The second period occurred between 1985-94 and saw communication practitioners place greater emphasis on factors such as source credibility, simplicity and clarity of message, and an effective use of message channels. Unlike during the first period, practitioners began to recognise the importance of receivers' perception of risk and crisis situations. Technical aspects of risk during this time were communicated in one direction from experts to government and then to the public. Communication practitioners in this regard focused on persuasive techniques in order to achieve their objectives. However, some elements of a 'two-way asymmetrical' model of communication management were introduced, whereby public attitudes were taken into consideration once experts had delivered their authoritative conclusions (Dora 2006: 170). Theorists such as Slovic and MacGregor (1994: 46) highlight the limited effectiveness of this type of risk and crisis communication approach and attribute a perceived lack of trust in a message source as a key consideration. Other theorists highlight how the 'two-way asymmetrical' approach utilised at that time generated a lack of real understanding of risk and crisis issues amongst concerned publics, resulting in the development of a 'knowledge gap' between 'experts' and 'nonexperts' (Cohn 2000: 166; Nohrstedt 2000: 140; Price 1989: 69; Gough and Hooper 2003: 2). Palenchar and Heath (2007: 122) describe the dominant view during this period as 'constructivism/relativism' where everybody's opinions are considered to have equal value. The paradigm adopted during this period is described by Dora (2006: 170) as 'a decisionist approach'.

Leiss and Powell's description of the third, post-1994, period identifies many of the shortcomings with both the 'dominant' and 'decisionist' approaches and highlights how contemporary practitioners now place considerable emphasis on organisationpublics dialogue, relationship and consensus building, transparency, meaningful participation and interaction in risk and crisis decision-making. The approach adopted during this time correlates closely with Grunig's 'two-way' symmetrical model and 
Murphy's (1991) 'mixed-model', previously described in Section 3.7.1., and is described by Dora (2006: 172) as 'a deliberative approach', which is a 'relational' form of communication and described earlier in Section 3.5.1.6. This approach is similar to Fischhoff's (1995: 138) final stage described earlier (p68). Theorists supporting such an approach include Sandman (1992: 3), Susskind and Field (1996: 37), Coombs (1999: 45), Reynolds (2002: 16), Price (1989: 179), and the Scarman Centre (2004: 3-28). Palenchar and Heath (2007: 122) state that this period is dominated by 'dialogue' where collaborative decision-making allows scientific opinion to become integrated into policies that are 'vetted by key public's values'. According to Dora (2006: 173):

A key element of the 'deliberative' approach is that risk communication is not treated entirely as an object of policy - it is not an exercise 'bolted on' at the end of a conventional, specialist-led process...instead it needs to involve dialogue about the definition and analysis, as well as the evaluation of any particular risk issue.

While the 'deliberative' model offers a useful approach to risk and crisis decisionmaking and communication efforts, many theorists are not convinced of its efficacy and highlight its shortcomings together with a number of inherent difficulties that need to be considered. For example, the need to incorporate pluralist views and cultural factors involves basing risk and crisis decisions, to a greater extent, on beliefs, values and ways of categorising the world rather than on objective information. Consequently, bias will unavoidably be encountered (Adams and Thompson 2002: 32; Ball 2002: 530). According to Seedhouse (1997: 77), a number of typologies of bias need to be taken into consideration, otherwise the 'deliberative' approach to risk communication can be undermined. These include biases such as 'necessary prejudice', 'blinkered prejudice' and 'reasoned prejudice'. Adams and Thompson (2002: 32) believe that 'sooner or later prejudice has to enter into any risk management decision process... because decisions are about facts but also about beliefs and preferences...therefore, whatever decision is made, there will always be grounds for dispute.' The cultural interaction of organisations and publics is likely to pose a further difficulty. Elliot and Smith (1993: 210) describe six factors in a 'cultural web' that the communication practitioner needs to consider. These are rituals/myths, symbols, power structures, organisational structures, control systems and routines. As will be discussed later in this dissertation, these six factors are used 
to explain why apparently good risk and crisis ideas, management, communication or messages fail to transfer effectively across cultural borders. A further difficulty is highlighted by Stern and Price (1989: 115), who describe how the different participants in the risk communication process may have unequal access to key information as a result of knowledge deficits or cost factors. Finally, consideration has to be given to potential difficulties for the communication manager in relation to the dispersion of responsibility and accountability following decisions taken using a 'deliberative' or 'dialogical' approach to risk and crisis communication (Stern and Price 1989: 112).

Taking the shortcomings of all three paradigms, described in Section 3.7.4, a number of theorists emphasise 'a middle road' strategy when deciding on an effective approach to risk and crisis communication issues. According to Adams and Thompson (2002: 34), people are as much exercised by practical and economic aspects of societal concerns as they are by engrained values. Ball (2007: 562) warns of the dangers of policy-making based solely on 'open discourse' to the exclusion of what can also be learned from quantitative risk assessment and cost-benefit analysis.

Consequently, risk communication practitioners should be cognisant that while the dialogical approach has clear benefits in terms of empowering members of the public, gleaning useful information, and achieving better 'buy-in' for decisions taken, the 'expert' opinion has a particularly important role in the risk communication process. Ball (2007: 562) cautions in the context of the 'dialogical' approach that, 'the processes by which such issues are incorporated into decision processes are still experimental and very few have been subject to rigorous evaluation'.

Many of the issues discussed in this section are equally germane to major emergency communication management and as a consequence will be discussed later in Chapter 5.

\subsubsection{Models of risk and crisis communication}

The study of risk communication has evolved from a number of theoretical models that have been proposed and describe how humans perceive and react to risk. 
Included amongst these models is Renn's (1998) model that specifies the different criteria employed in order to analyse and prioritise risk taking into account a number of major theoretical approaches (Renn 2008: 24). Additionally, Funtowicz and Ravetz (1992) offer a model that measures risk based on two key parameters, systems uncertainties and decision stakes. The type of communication approach adopted would be chosen based on where the risk lies on their model.

The 'risk perception model' developed by Covello, Wojtecki and Hyde (2001: 386), describes the complexity of assessing the perceptions surrounding the risk of death or serious injury. Coombs' (1999) normative, three-staged risk and crisis communication model describes the critical elements, both strategic and tactical, that communication practitioners need to consider for each stage. Hale's layered 'crisis communication architecture' model (1997: 238) describes six functions that are necessary in order to effectively support crisis response communication. Finally, Covello, Wojtecki and Hyde (2001: 388) 'trust determination model' highlights the key trust determination factors that are essential for effective risk and crisis communication.

These models provide a useful basis for an analysis of the theory and models of major emergency communication that are examined in detail throughout Chapters 5,6,7 and 8. However, these models do not offer much of an insight into the principles of strategic communication management. Consequently, in Chapter 4, a concise analysis is provided into this subject, which is a useful introduction to the discipline of the core subject of this dissertation, strategic major emergency communication management.

\section{In summary}

Chapter 3 thus demonstrates that communication theory is a relatively recent field of endeavour that continues to evolve. While the work of theorists such as Lippmann, Lasswell and Schramm, in the earlier part of the twentieth Century, provides a useful foundation for contemporary major emergency communication, the rhetorical-based, one-way, top-down approach is shown to be deficient in many respects. In keeping with contemporary theories of major emergency management, described in the last chapter, where response agencies plan and communicate with rather than for 
communities, the relational-based, horizontal, dialogical approach to communication, which originated from developmental and participatory communication theory, is preferred. As Fischhoff (p65) says, 'all we have to do is make them partners'. Theorists such as Grunig and Murphy propose the two-way dialogical approach as their preferred models of communication management. However, a number of theorists have also identified a number of shortcomings in this two-way, dialogical approach also and consequently further testing and evaluation is required. When considering communication and communication management from a strategic perspective, it will be shown in the next chapter that theorists consider the dialogical approach an important element of the strategic process. 


\section{STRATEGIC MANAGEMENT OF COMMUNICATION}

\subsection{Introduction}

Organisations do not exist within a vacuum but are obliged to co-exist, interact and survive with their external environment. Many changes within contemporary society require organisations to make sense of a turbulent, complex and sometimes threatening world. Because of this, organisations are well advised to devise systems for anticipating and solving the 'wicked' problems with which they are confronted on a daily basis (De Wit and Meyer 2004: 28). Organisational survival is based on its effective ability to monitor, interpret and respond to the many and diverse problems that threaten its survival. Since the 1950s, organisations have engaged in a process of strategic management in order to help them to survive in rapidly changing environments and also to succeed in achieving their goals. The processes of strategic management have evolved and expanded to include other diverse areas such as communication. In this chapter, it will be shown that a growing base of literature exists supporting the pursuit of strategic communication management. With the everincreasing numbers of post-graduate courses available in Masters of Business Administration (MBA) and Masters of Business Science (MBS) at third level institutions in Ireland, sourcing textbooks on strategy management is straightforward.

Lindeborg (1994: 5) emphasises the link between 'excellent' communication and strategy, stating:

Excellent communication is communication that is managed strategically, meets its objectives, and balances the needs of the organisation and the needs of key publics with two-way symmetrical communication. 


\subsection{Overview of strategy and strategic management}

\subsubsection{Strategy}

The term strategy derives from the Greek word strategia, meaning 'generalship'. Sun Tzu's The art of war, written in $500 \mathrm{BC}$, is regarded by many 'as the first treatise on strategy' (Grant 2008: 14). As an academic field of endeavour, however, the subject of strategy is relatively new. According to Kleiner (1996: 43), researchers during World War II spent considerable effort attempting to provide solutions for dealing with complicated environments. At the end of the war, these researchers brought strategy theory into the business environment. Their strategy skills came to good use in the 1950s and 1960s when senior executives were experiencing increasing difficulty in controlling and coordinating businesses that were expanding rapidly in terms of size and complexity. To overcome these difficulties, elite business schools in the US, such as Harvard, developed models of strategy and strategic corporate planning that were quickly adopted by large businesses. As a consequence, many useful texts started to appear.

Steiner (1969: 23) considers strategic planning as a plan, 'tangible evidence of the thinking of management'. However, during the 1980s shortcomings were identified in the strategic planning approach, in particular a belief that it resulted in an overly rigid mindset. According to Hill and Jones (2001: 22), organisations began to recognise that many successful realised strategies can emerge through a process of 'serendipity rather than through a planned process'. Consequently, organisations began to adopt a strategic management approach, rather than merely relying on a strict planning model. Today, large organisations place considerable emphasis on the external environment, business ethics, competing for standards and strategic innovation (Grant 2008: 18). However, despite the change of focus from the concept of strategic planning to strategic management, according to Hill and Jones (2001: 23), '...planning is an essential element of the strategic management process; even its most vocal critics concede that it has an important role to play'. 
Strategic management is the process of "making and implementing strategic decisions' (Asch and Bowman 1989: xiii). Mintzberg and Quinn (1991: 5) describe it as a pattern or plan that 'integrates the organisation's major goals, policies, and action sequences into a cohesive whole'. According to Grant (2008: 17) strategic management is the 'means' by which individuals or organisations achieve their objectives. This includes 'the plans, policies and principles that guide and unify a number of specific actions...not just the detailed actions' (ibid.).

\subsubsection{The strategic management process}

The strategic management process has been discussed in detail in a great number of texts over the past two decades. Many of the texts studied as part of this research provide relevant and self-explanatory diagrammatical models. In particular, by DeWit and Meyer (2004) and Grant (2008) use clear diagrams in order to explain concepts such as the strategy process, strategy formation and the common elements in successful strategies. Additionally, a number of texts were consulted in order to chart the evolution of strategy from World War II to the present day.

When it comes to strategic management, 'two words seem to pervade the literature, mission and environment' (Grunig 1992: 119). Mission is defined as, '...a statement of purpose - what the organisation hopes to achieve over the long term.... a pointer to the overall direction' (Grant 2008: 21). Robbins describes the environment as 'those institutions or forces that affect the performance of an organisation, but over which it has very little control' (Robbins 1990: 150). Van der Hiejden (1996: 33) introduces a third important element of strategy, 'uncertainty about the future' and says it is one of the main reasons why strategic management is needed. Finlay (2000: 9) describes uncertainty as an organisation's 'residual lack of knowledge...including the knowable and the intrinsically unknowable'. Schoemaker (1993: 193) believes that uncertainty can be positive as '...it stimulates reinvention, challenges traditional approaches, and fosters advanced anticipation, flexibility in strategies and continuous monitoring'.

As a result, the process of strategic management was developed in order to deal with the issues surrounding an organisation's mission, environment and uncertainty. As will be shown throughout this dissertation (particularly in Sections 4.3 and 4.4), a 
growing body of evidence exists supporting the premise that a well conceived and implemented strategic communication programme can greatly assist the organisation to interact with its environment, particularly during times of uncertainty.

Mintzberg and Quinn (1991: 15) emphasise that the strategic management process is a framework, as distinct from a hierarchical and structured plan. The process allows an organisation to 'get from where it is, to where it wants to be' (ibid.). For the purpose of analysis, this framework is best discussed in terms of sequential stages. These stages have been identified in models by a number of theorists, including Thompson and Strickland (1995: 3), Grant (2008: 7) and Johnson, Scholes and Whittington (2008: 12). These theorists propose a number of sequential steps that should be taken by strategy managers and include the provision of a long-term direction or mission, converting the mission into specific measurable objectives, crafting a strategy and plan, implement the chosen strategy, and evaluating the performance and correcting elements as necessary. What is noticeable about many of these models is that the type and sequence of steps proposed are quite similar.

In their strategic model, De Wit and Meyer (2004: 7) describe three distinct aspects of the strategy process: strategic thinking, strategy formation and strategic change. According to the authors, strategic thinking involves identifying what the problem is, diagnosing the nature of the problem, conceiving how best to address the problem, and implementing actions to be taken. Friend and Hickling (1997: 10) describe how decision-making is made more difficult by particular uncertainties that exist in the both the internal and external environment. To overcome these problems, they emphasise that organisations needs more information through ongoing research and analysis; more coordination through liaison and planning; and that goals and objectives need to be clearly stated.

In relation to the second element of De Wit and Meyer's model, 'strategy formation', this process is considered from two distinct perspectives. Firstly, 'strategy formulation' where intelligence is gathered and analysed and transformed into a definite plan or 'intended strategy' (as Mintzberg (1994: 31) terms it the 'grand strategy'). Secondly, a process for dealing with unplanned actions that arise because of forces in the environment and uncertainty and causes the intended strategy to 
'emerge' into an actual or 'realised strategy' (De Wit and Meyer 2004: 105). The main 'strategy formation' activities as described by De Wit and Meyer are shown in Figure 4.1 as follows:

Figure 4.1: Strategy formation activities

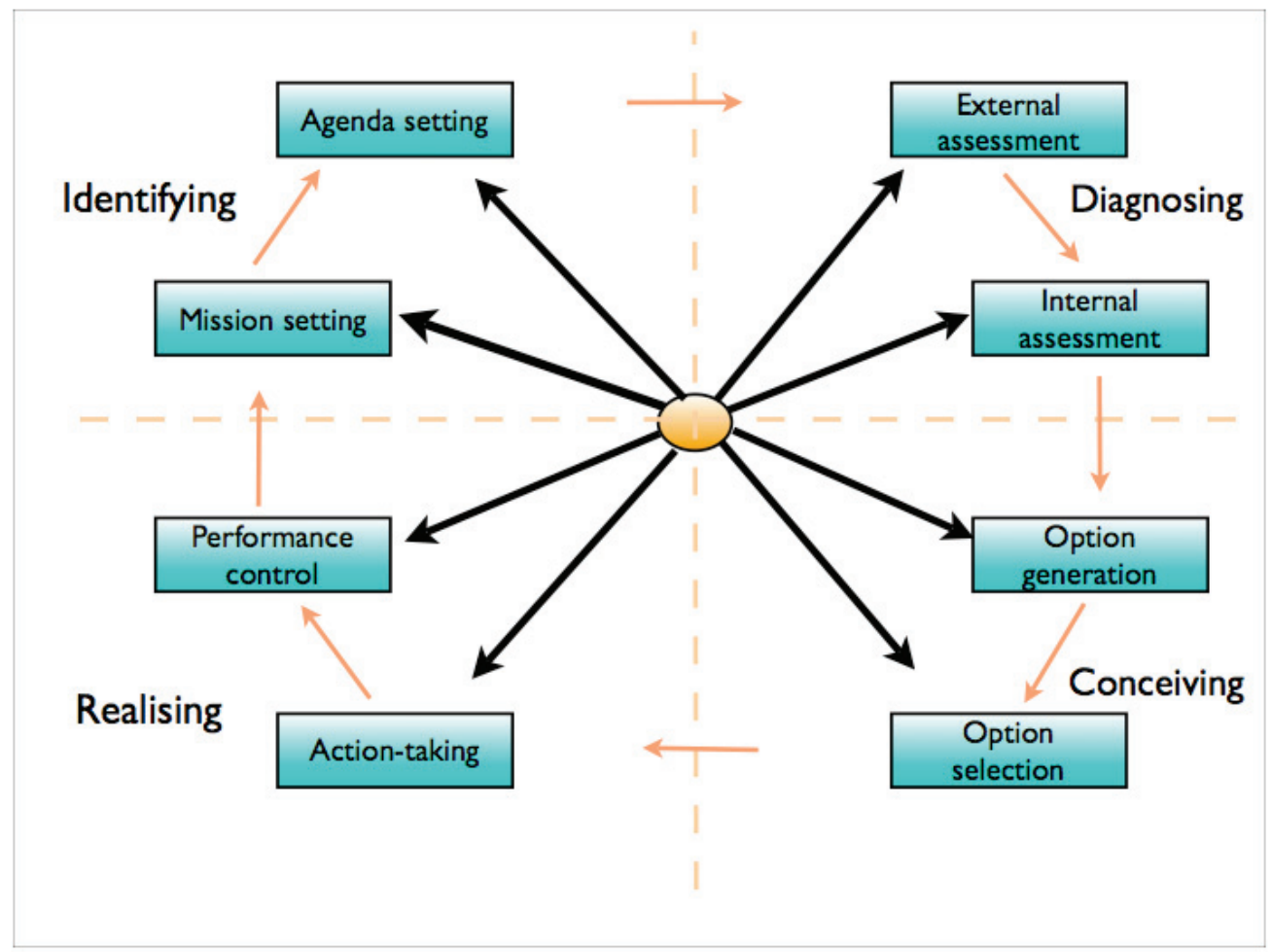

Source: (De Wit and Meyer 2004: 106)

Taking the different elements of De Wit and Meyer's model in sequence, mission setting involves outlining the purpose that the organisation wishes to serve or what it wants to achieve. Agenda setting helps the organisation to focus on specific strategic issues. An assessment of the external environment helps to identify opportunities or threats from outside the organisation and is generally done through research and analysis. Tools such as a 'strengths, weaknesses, opportunities and threats' (SWOT) model, a 'political/legal, economic, socio-cultural, and technological analysis' (PEST) model (Bowman 1990: 35), a 'Boston consulting group matrix (BCG) (De Wit and Meyer 2004: 299), and a 'quantitative strategic planning matrix' (David 1993: 12), prove useful in this regard. Assessing the internal environment is a process 
of analysing the capabilities and functioning of the organisation. The actions of option generation and selection involve going through a process of strategic thinking and decision-making, and then choosing an optimum course of action. Action taking involves implementing intended actions in order to achieve realised actions. This includes the strategic planning process, which is described as:

The systematic process through which strategic thinking is formalised and plans devised to support the strategy implementation (Finlay 2000: 16).

The implementation of performance controls involves evaluation of the implemented actions and assessment of success or failure of the realised strategy. It will be shown that the strategy formation process is an extremely important function during the preparedness and planning stages for major emergency situations.

De Wit and Meyer's third aspect of the strategy process is strategic change. This involves changing the configuration of the organisation and the way it operates in order to meet the challenges posed by its environment. This can involve changes to the organisation's structure, processes and culture (De Wit and Meyer 2004: 166).

\subsubsection{Strategic versus tactical approaches}

Tactics relate to the actions contained within a strategic plan that are employed to make the plan a success, and to assist the organisation in accomplishing the designed strategy. Tactical planning is an integral part of strategic planning and as such both should work in harmony. Highlighting the benefits of implementing a strategic approach rather than a tactical approach, Grant states:

Strategy is the overall plan for deploying resources to establish a favourable position; a tactic is a scheme for specific action. While tactics are concerned with the maneuvers [sic] necessary to win battles, strategy is concerned with winning the war (2008: 7). 


\subsubsection{Strategic management and cultural theory}

While a growing body of evidence supports the strategic management process, it needs to be considered within the context of cultural theory as discussed earlier in Section 3.4.3. Johnson and Scholes (1988: 113) acknowledge the significance of the cultural and political systems of an organisation and highlight 'the growing awareness of the central importance of these issues in understanding strategy formulation and independence'. Additionally, they identify a 'cultural web' of factors 'which preserve and sustain commonly held core-beliefs - the recipe' (ibid: 41). The recipe includes factors such as power structures, control systems, rituals and myths, symbols, routines and organisational structures, and is considered a set of subconscious basic assumptions and beliefs, shared by everyone within the organisation, which defines how business is conducted. According to Johnson (1992: 208), an analysis of factors in the web allows managers to 'discover' the nature of their organisation, the strategy that they are following and why it may fail to transfer across cultural borders. This is an important consideration in the context of major emergency communication management and will be discussed later in this dissertation.

\subsection{Strategic communication management}

Unfortunately, the literature referring to strategic communication management is not as well developed as that relating to organisational strategic management. What is noticeable is the predominance in the field of collaborations by a relatively small group of theorists, including James and Larissa Grunig, David Dozier, Glenn Broom, Scott Cutlip, Allen Center and Denis Wilcox.

As mentioned earlier in Section 4.1, many organisations are obliged to operate and fulfil their missions and goals in very turbulent environments together with a great deal of uncertainty as to their future. This is particularly relevant when dealing with 'strategic constituencies' that are described as:

Elements of the environment whose opposition or support can threaten the organisation's goals or support or help to attain them (Grunig and Grunig 2000: 306). 
Theorists and practitioners in strategic management have developed many models in order to assist organisations to deal with, and overcome, their difficulties. As was discussed in Chapter 2, communication management provides an effective means of positively interacting with and influencing an organisation's internal and external environments. Cutlip, Center and Broom (2000: 6) describe how communication management can 'establish and maintain a mutually beneficial relationships between an organisation and the publics on whom its success or failure depends'. Grunig and Grunig (2000: 310) discuss how communication management plays a critical role in strategic management by helping the organisation to identify the most important constituents in the environment and building good relationships with them through communication. However, as will be discussed in Section 4.4, communication management is more effective when the process itself is managed strategically, thereby allowing optimum support to the organisation in achieving its mission. However, according to many key theorists, in order for communication management to be fully supportive and optimal, the senior communication practitioner must be at the centre of decision-making within the organisation (Grunig 1992: 155; Plowman 2005: 131; Darrell 2003: 20; Cutlip, Center and Broom 2000: 170).

Consequently, strategic communication management can be defined as:

A deliberate, planned, sustained and systematic series of communication programmes that supports the organisation's strategic management efforts (Wilcox and Cameron 2005: 23).

According to Dozier, Grunig and Grunig (1995: 85), communication becomes strategic when it ' ... assists top management in managing the pursuit of its purpose and direction, coincident with managing relationships with key publics in the organisation's environment'.

Plowman (2005: 132) believes the 'strategic' part of strategic communication management means 'accomplishing goals and solving complex communication problems'. Webster (1990: 18) concurs, stating that in order to be considered strategic, communication management must at a minimum be '...aligned with the corporate vision or mission and substantially contribute to achieving the organisation's objectives'. However, it must be acknowledged that there may be 
conflict between the goals of the organisation and its stakeholders and publics. This creates an additional challenge for the communication practitioner. Significantly, Grunig and Grunig (2000: 308) emphasise that strategic communication management involves much more than a mere 'set of communication tactics supplied by communication technicians'. Plowman (2005: 133) agrees stating:

Participation with strategic management elevates public relations from its typical tactical role of reacting to events to a more proactive, responsive role of anticipating and reducing emerging conflicts.

Other important elements and functions of strategic communication management include boundary spanning the open systems environment with strategic stakeholders, strategic thinking, taking a long-range view of the effects of organisation actions and encouraging joint fact finding and intelligence sharing (Plowman 2005: 132).

\subsubsection{Tactical communication management}

In many organisations the communication practitioner is often relegated to that of a tactical or functionary role. This relates to a role that is considered non-strategic, described by Dozier, Grunig and Grunig (1995: 54) as that of a 'communication technician... where the practitioner merely implements the decisions of others...a received role'. Grunig (1992: 121) proposes that communication managers who operate at the tactical level are restricted to the 'application of technique rather than the formulation of policy'.

A tactical approach to communication management involves pursuing the planning of narrow short-term advocacy techniques and the implementation of individual programmes. Tactical communication managers rarely engage in any strategic research or evaluation (Daugherty 2003: 21). Consequently, they have no basis for understanding fully the perceptions, opinions, needs and behaviours of publics, or of the determination of subsequent message effects (Grunig, Grunig and Dozier 2002: 255). Communication programmes adopting this approach may be 'missing any sense of purpose...lacking in a sense of direction' (Dozier, Grunig and Grunig 1995: 59). 
Certain theorists have cited particular reasons why practitioners adopt tactical approaches towards communication management rather than strategic ones. These reasons include amongst others, the practitioners lack of knowledge or experience to play the strategic communication managers role (Grunig, Grunig and Dozier 2002: 255); an absence of formal communication education (Carrington 1992: 45; Grunig 1992: 439); the culture and attitude towards communication within the organisation, particularly by senior decision-makers (Dozier, Grunig and Grunig 1995: 148); and the relative ease and comfort of routinely pursuing a 'historicist', or 'the way we have always done things here', approach (Steyn, Green and Grobler 2001: 8).

\subsubsection{A model of strategic communication management}

One of the most comprehensive normative models of strategic communication management was developed by Grunig and Repper through their 'Excellence Study' (Grunig 1992: 124). This model is significant in that it integrates and highlights the role of communication within the wider strategic management context. For example, the model demonstrates how communication is critical in identifying stakeholders, publics and issues that can impact on the organisation's survival or reputation. Significantly, the model also places particular emphasis on the role of communication in the ongoing monitoring and subsequent understanding of the organisation's external environment. It thereby provides critical information that can allow the overall mission to be adjusted if necessary. As will be demonstrated in Sections 6.4.1.1 and 7.3, environmental scanning and information-gathering is extremely important for the effective identification and management of major emergency situations.

The model consists of three distinct stages, the 'stakeholder stage', the 'public stage' and the 'issues stage'. At the 'stakeholder stage', the organisation and the stakeholders have a particular relationship. The behaviour of one to the other will have an influence on how each is likely to act and possible decisions that may be taken by either side. According to Repper, the organisation needs to understand who these stakeholders are, and should engage in continuous research and environmental scanning in order to assess the consequences of its decisions on these stakeholders. Objective actions such as these can assist the organisation in identifying and 
evaluating issues of mutual or conflicting consequences between it and its external environment. It will be shown later in this dissertation, in Sections 5.3.2, 7.5, and 8.3 that the lead response organisation needs to be able to identify, understand and engage in meaningful two-way dialogue with its stakeholders and publics in order to efficiently prepare for and respond to a major emergency situation.

At the second stage of the model, 'the public stage', the goal of strategic communication management is to segment these publics in order to allow individual groups, supporters, neutral or opponents, to be clearly identified. Thereafter the strategic communication practitioner should involve the identified publics in meaningful dialogue surrounding the decision-making process in order to mitigate potential conflict. This is similar to the broad-based deliberative process that will be shown to be an extremely important element of strategic communication management.

At the third stage, the 'issues stage', publics organise and create issues out of problems they perceive. In relation to major emergency situations, an example of a potential issue is the reported lack of sufficient anti-viral drugs to treat the entire population in the event of an influenza pandemic. This is likely to provoke protests by certain members of the public should a pandemic become imminent, thereby creating 'publics' (mentioned earlier in Chapter 1). The role of strategic communication management is to anticipate these issues and manage the Irish Health Service Executive's (HSE) response to them. As issues develop, the media play a more significant role in their generation and promulgation. The use of communication research is important at this stage. In order to deal with different categories of publics, the strategic communication manager may require the use of different approaches, such as inter-personal communication using dialogue and negotiation to deal with activist publics, and the use of the mass media to reach the wider public.

Repper, cited in Grunig (1992: 124) emphasises that communication programmes need to be developed for different categories of stakeholders and publics at each of the three stages. Other critical elements included in the model are: the setting of formal goals and objectives for the communication function; planning formal programmes and campaigns to accomplish each objective; and conducting evaluation 
in order to assess the effectiveness of programmes in terms of meeting their objectives and in the reduction of conflict produced as a consequence of effective decision-making. Each of these elements will be discussed in greater detail throughout the dissertation.

Offering a revised version of this model, Grunig, Grunig and Dozier (2002: 145) incorporate an important additional element, the 'interconnectedness' and interdependence that exist between decisions taken and outcomes. The model describes management's decisions and how these are likely to generate specific 'consequences' on the likely 'behaviour' of stakeholders and publics alike. Their process can result in a feedback loop to management from stakeholders in order to allow a revision of the original decision. However, if the decision is poorly handled, the subsequent stakeholder 'behaviour' is likely to generate 'issues' and possibly 'crises' that can impact on the organisation. At this stage of the cycle, how these 'relationships' are managed will affect and influence the 'reputation' of the organisation and is likely to impact on whether or not the organisation achieves its 'goals'. It will be seen later that decisions taken by major emergency planners, such as failing to protect levees in New Orleans, generated 'issues' for certain stakeholders who provided feedback to the authorities. Eventually, the decision not to protect the levees resulted in a full-blown crisis, the flooding of New Orleans. This model is presented in Figure 4.2 over 
Figure 4.2: Strategic communication management framework

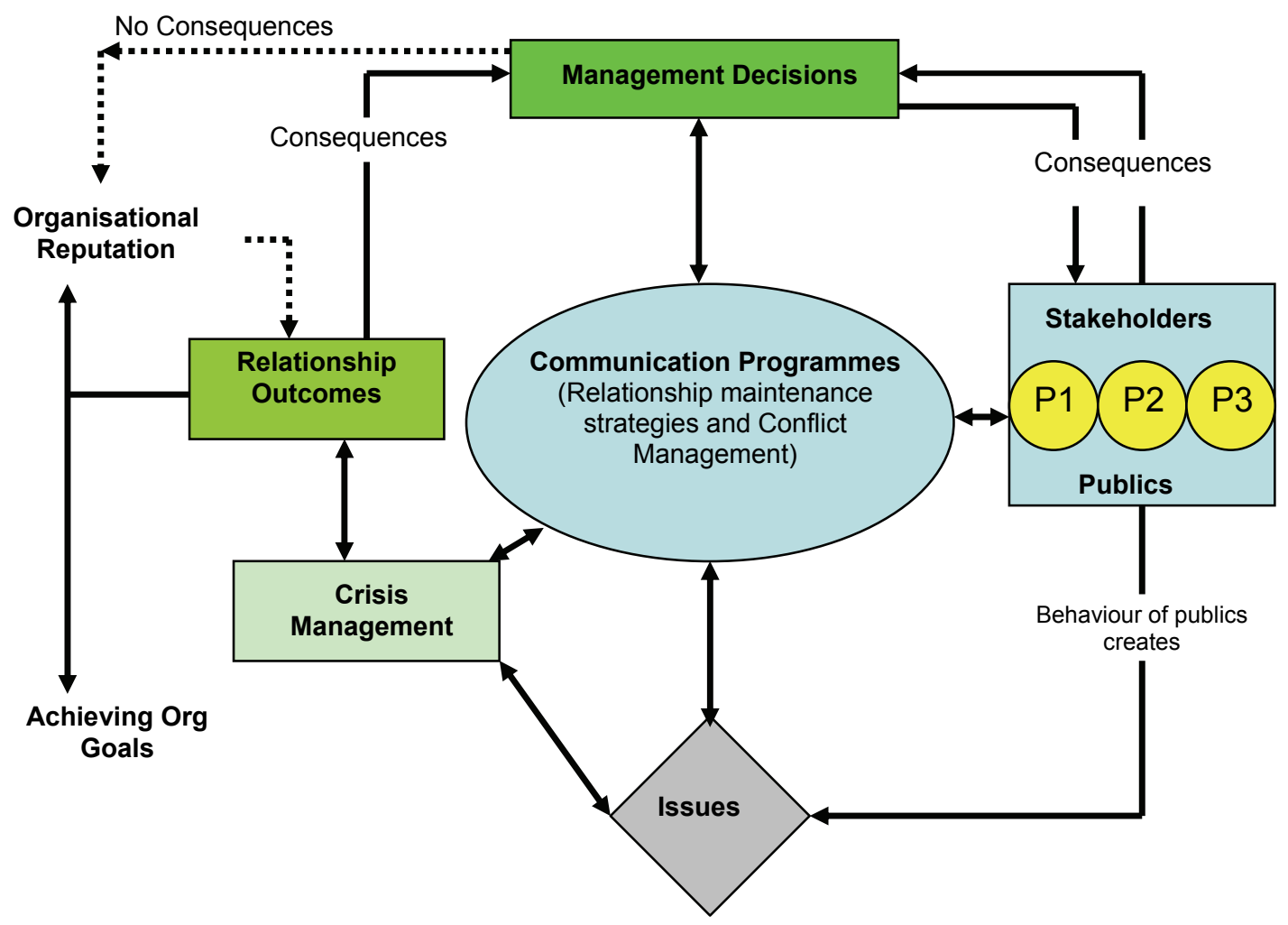

Source: (Grunig, Grunig and Dozier 2002: 145)

\subsection{Key elements involved in strategic communication management}

Through studies such as the 'Excellence Study' (Grunig 1992) and later work by theorists such as Larissa Grunig, David Dozier and Glen Broom, a number of key elements have been identified that can be associated with excellent communication management. These key elements provide the basis for a number of strategic principles that underpin strategic communication management. It will be demonstrated that these principles also underpin the strategic management of major emergency communication, which will be discussed in detail in Chapter 5. The key principles of strategic communication management are described below. 


\subsubsection{Culture and organisational structure}

The underlying culture, structure and world-view of an organisation will have a significant bearing on the approach taken towards the management of its communication process. Norman, Stuart-Black and Coles (2006: 110) describe communication as 'a vein that runs through every tier of the organisation'. According to Grunig (1992: 591), societal culture can impose a particular world-view on an organisation and generate presuppositions about the way communication should be managed. In order to implement an 'excellent' communication management process, it may be necessary, in the first instance, to consider the cultural environment within which the organisation operates. This was briefly discussed earlier in Sections 3.4.3 and 4.2.4, and it is recognised that the task may not be as simple as it appears. Cultural differences between individuals and societies may result in similar information being received and processed in different ways, resulting in varying types of behaviour. Consequently, theorists such as Smiramesh and White (cited in Grunig 1992: 610) and Dozier (cited in Dozier, Grunig and Grunig 1995: 170), describe how communication practitioners in one country may require a different approach than their counterparts in other countries. Secondly, according to Frosdick (1995: 55), exploring cultural differences provides useful explanations as to why apparently good strategies sometimes fail and 'encourages an appreciation of the validity of alternative points of view'.

Additionally, as a result of the nature of the culture and structure of the organisation, changes may be required. Important changes that can be brought about in this regard include, the way that information flows both vertically and horizontally throughout the organisation, how decisions are reached, and a willingness to engage in scientific communication techniques such as research and evaluation. A change in organisational culture or structure, such as this, can facilitate the development and implementation of a genuine two-way symmetrical-model, or mixed-model of communication, between the organisation and its external environment (ibid.). 


\subsubsection{Management-by-objectives}

The process of 'management-by-objectives' (MBO) involves senior decision-makers establishing and agreeing a series of goals and objectives within the organisation. This creates a focus for management of achieving stated aims and attaining the best possible results from available resources. MBO also provides a useful means of evaluating managers' abilities to achieve specific organisational goals within certain timeframes and operating budgets (Hill and Jones 2001: 429; Seitel 2004: 81). Theorists identify four steps that need to be taken in the MBO approach for strategic management: The establishment of overarching objectives; the development and issuing of particular assignments, plans, procedures and protocols; establishing clear, specific, and measurable objectives for tactical activities; and documenting results to measure performance in order to instigate corrective action (Nordlund 1994: 17; Reynolds 2002: 20; Ridge 20049(c): 6).

According to Cutlip, Center and Broom (2000: 375), when organisations employ MBO to guide the strategic communication management process, the focus shifts from producing communications, or focusing merely on the communication process, to achieving results or impact. This makes the strategic communication practitioner more accountable for achieving their aspects of organisational goals and ensures that the focus of the strategy is to achieve overall effectiveness as well as efficiency (Lindeborg 1994: 5).

A number of theorists, including Hon (1998: 105), Pieczka (2000: 213) and Grunig (1992: 262) have stressed that communication goals must be clearly aligned to the overarching goals of the organisation. Temple (2002: 33) agrees and describes the alignment of goals as a 'clear tight-fitting nexus'. Alignment of the communication and operational goals and objectives will ensure that the communication process assists, supports and complements the overall vision of senior decision-makers, rather than operating as a stand-alone function. Communication goals and objectives must also be clearly defined and understood by all, be operationally attainable, be quantitatively measurable, and be oriented toward overall improvement. 


\subsubsection{Strategic communication planning}

Many theorists such as Higgins (1979: 9) and Daugherty (2003: 21) emphasise that strategic communication planning is an important element of, and complementary to, the overall strategic communication management process. According to Dozier, Grunig and Grunig (1995: 85), 'senior management engage in strategic communication planning when they make strategic decisions in a proactive manner'. Temple (2002: 32) believes, '... sound strategic communication planning is the critical success factor in developing effective crisis communication strategies - and must never be neglected'. Fearn-Banks (1996: 24) contends that the value of strategic communication planning derives largely from the detailed analysis and strategic thought that surrounds the process rather than the end product. Daugherty (2003: 21) believes that care must be taken, as the process of strategic communication planning is often overly 'structured and hierarchical' and can therefore hinder positive action during events that have not been planned for.

Many theorists, including Smith (2005: 220) and Cutlip, Centre and Broom (2000: 341) propose models that describe the strategic communication planning process. The process generally follows a distinctive phased pattern, with a series of steps outlined for each particular phase. While the content of each model is remarkably similar, the sequence and timing of steps may vary from model to model. These steps generally include: defining the communication problem using research and analysis; using techniques such as 'brainstorming' to develop plans and programmes to deal with each communication problem; allocating adequate resources and budgets for each element of the plan; deciding on appropriate deadlines and a clear delineation of responsibilities for each particular element of the plan; implementing the plan through action and communication; and finally, the strategic evaluation of each of the communication programmes in order to determine its success of failure.

Central to a successful communication planning process is the ability to make decisions strategically. This involves choosing an optimal course of action from a range of possible alternatives. According to Clemen and Reilly (2001: 6), when making important communication decisions, decision-makers can utilise modelling tools such as decision trees in order to aid analysis. Important variables such as 
uncertainty need to be factored into the process. Other tools, such as probability analysis, can assist in this regard.

Many barriers exist to the conduct of effective strategic communication planning. These include a lack of strategic communication knowledge or experience, inadequate consultation and deliberation, a failure to agree clear goals and objectives for the implementation of the programme, inadequate or unrealistic time frames, unsuitable processes of internal clearance, a lack of coordination, and the absence of flexibility (Temple 2002: 32).

\subsubsection{Strategic communication research}

According to the Price (1989: 13), one of the key characteristics that differentiates strategic communication management from tactical management is that practitioners undertake ongoing, systematic and strategic communication research. Broom and Dozier (1990: 85) define this type of research as '...the controlled, objective and systematic gathering of information for the purposes of describing and understanding'. According to Ehling (1985: 236), any communication management that is not based on strategic research is likely to be 'flawed'.

Many theorists have identified a number of different approaches to communication research. Using a continuum, Broom and Dozier (1990: 14) describe one category of non-strategic communication practice that utilises the 'no-research-approach' or 'seat-of-the-pants' approach. This approach is considered to be individualistic, subjective and personal, 'with little use for social or behavioural research' (Grunig 1992: 162). Further along the continuum, Austin, Pinkleton and Dixon (2000: 82) describe 'informal research' where programmes are developed using an unscientific or unrepresentative approach to the discipline. At the optimal end of the continuum, Grunig (1992: 162) describes a 'scientific management' approach to communication research. Using this method, strategic practitioners engage in different types of communication research, that includes 'formative', 'programme' and 'summative' research. 
Adopting a scientific approach to research, the strategic communication manager can obtain much vital information necessary to make complex decisions in relation to goal setting, programme direction, message emphasis, and budget allocation (Simpson 1992: 27). Strategic communication research also allows decision-makers to have greater confidence that their findings reflect reality, not just decision-makers' perceptions of reality (Broom and Dozier 1990: 4).

Significantly, conducting strategic research allows communicators to identify and segment the general public into groups 'whose members are bound by mutual interests, concerns and characteristics' (Fearn-Banks 1996: 13; Wilcox and Cameron 2005: 130). According to Grunig (1992: 127) this is extremely important as the general public 'is a contradiction in terms' as it cannot be considered a homogenous mass. Many contemporary theorists are in agreement that strategic communication research should be conducted early, and thereafter should be sustained throughout all stages of the communication life cycle.

\subsubsection{Strategic communication evaluation}

Rossi and Freeman, cited in Cutlip, Center and Broom, (2000: 432) define strategic communication evaluation as:

A systematic application of social research in order to assess the conceptualisation, design, implementation and impact of communication programmes.

Theorists such as Lindenmann, cited in Hon, (1998: 112), Coombs (1999: 136), and Reynolds (2002: 7) contend that strategic evaluation should not be conducted merely at the end of a communication programme, but should be carried out 'regularly and systematically throughout all stages'. The strategic evaluation of any communication programme involves three distinct stages. These include, 'preparation evaluation' where critical preparatory tasks, such as the process of defining the communication problem and planning the communication programme, are evaluated (Cutlip, Center, and Broom 2000: 440). Once communication plans have been developed, 'implementation evaluation' allows a detailed assessment to takes place with regard to the implementation of prepared communication plans and how the communication 
processes are effected (Pieczka 2000: 214). Finally, communication practitioners can conduct an 'impact evaluation' where a comprehensive assessment of the outcomes and the impact of implemented communication programmes take place (Hon 1998: 106). However, in order to properly evaluate the effectiveness of a strategic communication programme, goals and objectives should firstly be established in order to provide a benchmark to work against (Hon 1998: 105; Daugherty 2003: 26).

\subsubsection{Building relationships}

Many of the definitions of strategic communication management relate to the development and maintenance of good relationships between an organisation and its external environment. According to Ferguson (1984: 1), the relationships between organisations and their publics 'should be the central unit of study for strategic communication managers'. Many theorists, such as Alesch et al. (2004: 2) and Covello and Sandman, cited in Wolbarst, (2001: 171), emphasise the importance of building and maintaining good relationships with groups that make up the external environment, especially the media.

In order to ensure good relationships between an organisation and its publics, it is necessary that the relationships be strategically managed (Lindenmann 1998/99: 19). This can be achieved through a number of critical steps beginning with the establishment of 'process objectives' (ibid.). This step involves the identification of stakeholders and publics and developing specific programmes in order to build and manage the relationship with each group (Ledingham 2000: 46). The establishment of 'outcome objectives' is the next key step where the quality of the relationship is measured following the implementation of the specific communication programmes (Hon 1998: 129; Grunig Grunig and Dozier 2002: 550). The perception of the strength of the relationship is also considered important and should be measured (Broom, Casey and Ritchey 1997: 95). According to Grunig (1992: 82), few communication managers 'study or measure the quality of relationships sufficiently', therefore the strength of the relationships is difficult to determine and improve. 


\subsubsection{Broad based deliberation}

A growing body of literature highlights how a strong participative culture and meaningful dialogue can greatly enhance communication flows from an organisation to its external environment, thereby improving levels of openness, transparency, trust and credibility. This 'dialogical' approach has been discussed earlier in Section 3.7.4. As will be discussed throughout this dissertation, particularly in Sections 5.3.2 and 9.4.4, enhanced dialogue and engagement is an important strategy for dealing with crisis and major emergency situations (Grunig 1992: 17; Stern and Fineberg 1996:118; Mileti 1999: 9; Susskind and Field 1996: 231; ILGRA 1998: 8).

This critical process has been described in a number of ways by a variety of theorists, including 'deliberative' approach (Dora 2006: 172); 'dialogical' approach (Palenchar and Heath 2007: 122); 'full participation theory' (Grunig, Grunig and Dozier 2002: 383); 'mutual gains approach' (Susskind and Field 1996: 37); and 'bridging social capital' (Putnam 2000: 19). Where members of the public are meaningfully engaged in complex issues where scientific analysis and input is required, the process is known as a 'broad-based analytic-deliberative approach' (Stern and Fineberg 1996: 71; Ball 2005: 25).

As was discussed in Chapter 3, in situations where complex or technical risk issues exist, communication practitioners are often reluctant to engage meaningfully with their stakeholders and publics in the 'broad-based analytic-deliberative' process when it is considered that the latter lack technical expertise and cannot be held accountable for decisions that they take (Kweit and Kweit 1984: 32). However, a balanced approach, described as 'a middle road' strategy (Adams and Thompson 2002: 34), is one where the views of the scientific community, including quantitative risk assessments and cost-benefit analysis, are taken into consideration together with the views and opinions of 'non-expert' stakeholders. As the process of engaging the public in dialogue is somewhat recent in the context of major emergency communication management, the theoretical and practical process will need to be rigorously tested in order to prove its efficacy. The dialogical approach to major emergency communication management will be discussed further in Chapter 5 . 


\subsubsection{Strategic communication knowledge: (Formal education and experience)}

Many theorists, including Grunig (1992: 439), Dozier, Grunig and Grunig (1995: 7), and Rose (2002: 15) place considerable emphasis on communication practitioners possessing requisite communication knowledge and experience in order to engage in excellent strategic communication management. According to Dozier, Grunig and Grunig (1995: 11), '... at the core of the sphere of strategic communication excellence is the knowledge base of the communication practitioners'. As mentioned earlier, one of the key elements that differentiates the role of strategic communication managers from that of tactical communication managers is the knowledge and experience to undertake the function of strategic communication manager. Without strategic communication knowledge, it is unlikely that the communication manager will be able to undertake important functions such as: implementing different models of communication (one-way, two-way and mixed-models); have the ability to contribute meaningfully to strategic policy decisions; provide communication programme alternatives; guide senior management through logical problem-solving processes (Dozier, Grunig and Grunig 1995: 39; Grunig 1992: 460); conduct strategic research, planning and evaluation throughout all stages of the communication life cycle (Stern and Fineberg 1996: 155); or manage communication in a society with rapidly changing demographic profiles (Rose 2002: 15; Fearn-Banks 1996: 10). Theorists such as Grant (2008: 159) and Dranove and Marciano (2005: 191) emphasise the importance of academic courses for gaining indepth knowledge in order to benefit the organisation.

\subsection{Strategic communication management during a major emergency situation}

A comprehensive examination of the literature dealing with the subject of strategic major emergency communication management reveals that the most useful data emanates from communication sections within government departments or agencies. Amongst the most notable contributors are the Federal Emergency Management Agency (FEMA), Centers for Disease Control (CDC) and National Response Team 
(NRT) in the US; the Civil Contingencies Secretariat (CCS), Inter-Departmental Liaison Group on Risk Assessment (ILGRA) and the Emergency Planning Society (EPS) in the UK; the Swedish Emergency Management Agency (SEMA) in Sweden, Emergency Management Australia; Public Safety Canada; and the International Federation of the Red Cross and Red Crescent.

By their nature, major emergencies are characterised by uncertainty, rapid change, confusion and doubt, where 'central values are threatened, and established authority structures are called into question' (Nohrstedt 2000: 139). It is sometimes questioned whether the theories, models and approaches of strategic communication management, mentioned throughout this dissertation, can be of any benefit when dealing with the complexities of major emergency situations. However, according to the US Centers for Disease Control (CDC), implementing a strategic major emergency communication management process in the short-, medium-, and longerterm:

...will put you back in control...map out how you operate...increase certainty amongst stakeholders through information...reduce isolation, develop allies... and identify resources required (Reynolds 2002: 45).

Additionally, Dyer (1995: 41) emphasises that a good major emergency communication management strategy 'can result in saving human lives'.

With the exception of a small number of guidance documents, very few texts exist that provide a comprehensive examination of major emergency communication management, from a strategic viewpoint, throughout all three stages. Much of the existing literature associated with the discipline consists of quasi-strategic models, together with tactical guidelines and checklists for the communication practitioner, particularly for the crisis / response stage of the major emergency life cycle. Despite this, a small number of theorists have provided reasonably comprehensive models that have proven valuable during this research.

One of the most useful models examined is one developed by Barbara Reynolds for the US Centers for Disease Control and Prevention, (CDC) based in Washington, D.C. (Reynolds 2002: 7). This model is based on a five-staged approach and 
comprehensively describes the key communication requirements throughout all stages of a major emergency life cycle.

Figure 4.3: Major emergency communication management life cycle

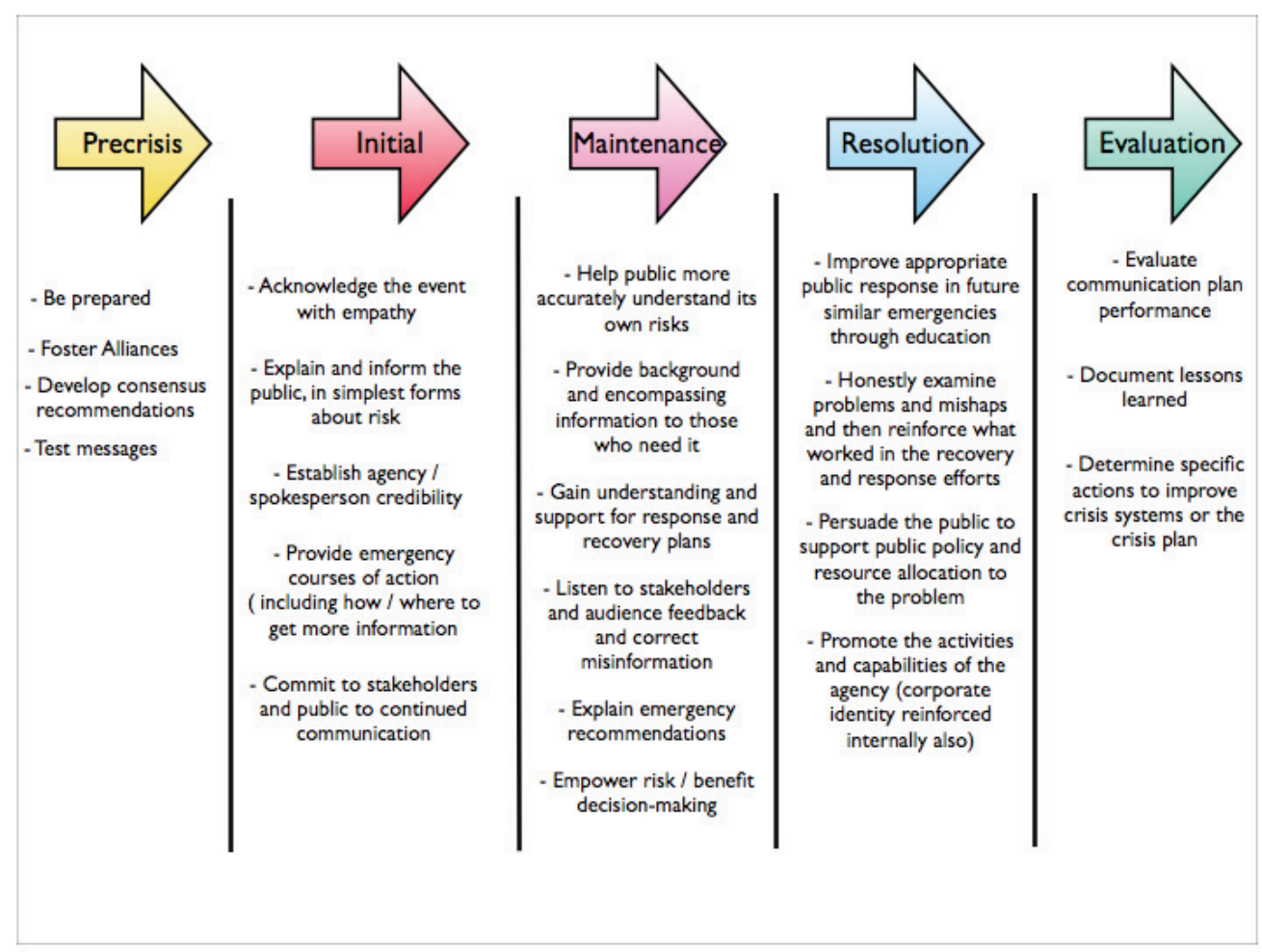

Source: (Reynolds 2002: 7)

Offering a useful normative model of strategic major emergency communication management, UK Resilience (2005: 28) incorporates the cyclical elements of strategic communication planning, along with many key aspects of the management process. Of significance within this model are the communication flows at different stages throughout the process, including the receipt of information at the problem formulation stage and two-way flows of information at the planning and decisionmaking stage. Additionally, the model emphasises approaching major emergency communication using a scientific perspective, making full use of communication research and evaluation. Such strategic approaches assist the major emergency practitioner to solve simultaneous complex problems in very difficult circumstances. 
Nordlund's (1994) descriptive 'Triangle Drama' model provides a very useful framework in order to analyse the interaction between three of the key participants involved in a major emergency situation, the government, its publics, and the media. The model provides a very comprehensive and useful description of the key communication functions that should take place throughout all stages with particular emphasis on research, evaluation, setting clear goals and objectives and developing a communication plan. Nordlund also analyses the key characteristics of the communication message and medium. In this regard he places particular emphasis on the importance of the media throughout a crisis.

\section{In summary}

Chapter 4 describes how the strategic management process was developed in order to allow organisations to interact effectively within an uncertain environment and to survive during turbulent times. The strategy process for organisations, as outlined in De Wit and Meyer's model, involves a number of critical actions including identifying problems and establishing goals, assessing the internal and external environment, establishing courses of action, taking objective action and evaluating progress and results. In the early 1990s, a number of communication theorists, including James Grunig and David Dozier, recognised the value of this process to the communication management function and developed models accordingly. They proposed that without such a strategic approach, the communication practitioner is obliged to engage in tactical communication, involving a narrow, short-term focus on individual programmes. A strategic approach, on the other hand, allows the communication manager to achieve aims in the longer-term, aligned to the overall corporate vision, and to deal with a range of complex problems simultaneously. It also provides a basis for the communication manager to understand fully the perceptions, needs, opinions and behaviours of key publics, through communication research and evaluation. Adapting the strategic communication model to the major emergency life cycle, theorists such as Reynolds and Nordlund propose models that can guide the communication function throughout the uncertainty and turbulence of a major emergency situation. While these models are useful, they are deficient in certain respects. Consequently, building on the strengths of each of the models discussed in Chapter 4, and considering data gleaned from the literature, a proposed 
framework for strategic major emergency communication management will be proposed in Chapter 5. 


\section{STRATEGIC PRINCIPLES AND THE MAJOR EMERGENCY COMMUNICATION FRAMEWORK}

\subsection{Introduction}

In the previous chapter, a number of models of strategic major emergency communication management were discussed. They provide a useful basis for understanding communication flows during a major emergency. Taking cognisance of the information contained within these models, together with other useful sources, a strategic major emergency communication management framework is proposed in Chapter 5. This framework provides the reader with a comprehensive guidance model in order to manage the communication function during all three stages of a major emergency life cycle. Such a detailed all-encompassing strategic model has heretofore been difficult to source. In the development of this framework, consideration has been given to many of the deficiencies that have been noted in existing strategic communication models. The proposed framework demonstrates how many of the critical strategic communication principles, drawn from organisational strategy, can guide the tactical functions of communication throughout all three stages.

This chapter identifies and analyses six strategic principles that underpin the management of communication throughout the three stages of the major emergency life cycle. It is shown that these communication principles encompass the entire framework and are ongoing and iterative, thereby creating ideal conditions within which an exceptional major emergency communication strategy can be developed and managed. Each strategic principle is discussed in turn and in no particular priority. In relation to one of the strategic principles, integrated communication, it is considered beneficial to examine both the strategic and tactical elements in this chapter, particularly as these functions span all three stages of the major emergency life cycle.

Before delving into the strategic principles, a brief description of the practitioners responsible for the communication function within the lead response organisation and 
the structure of the major emergency communication and information team (MECIT) is given.

\subsection{The major emergency communication and information team (MECIT)}

Coombs (1999: 63) describes the crisis communication team as 'a cross-functional group of people' that has been designated by the organisation to lead the strategic communication function throughout all stages of a major emergency. In an ideal situation, this team's central role is the co-ordination and management of both communication and information flows. Consequently, throughout this dissertation this team is referred to as the major emergency communication and information team (MECIT). In a major emergency situation, each of the other supporting response agencies should also provide their own communication teams. Depending on the scale of the major emergency, the MECIT from the lead response organisation may be obliged to establish a central location in order to coordinate the actions of each of the other MECITs involved. This central location is termed a communication and information management centre (CIMC) throughout the remainder of this dissertation. The CIMC is discussed in greater detail later in Section 5.2.12.6.

At the head of the lead MECIT is an experienced and knowledgeable major emergency communication practitioner, who should be a senior executive within the lead response organisation. A number of theorists, including Coombs (1999: 91), Marra (1998: 8), Daugherty (2003: 21), and Grunig (1992: 103) emphasise the importance of involving the lead communication practitioner of the MECIT in the strategic decision-making function at the highest level of the lead response agency. This ensures a prominent role for communication within the overall operational function and guarantees the setting of communication policy by communication experts rather than a mere implementation of others' policies. 


\subsection{Six strategic major emergency communication principles}

Figure 5.1: Proposed framework for strategic major emergency communication management - overview.

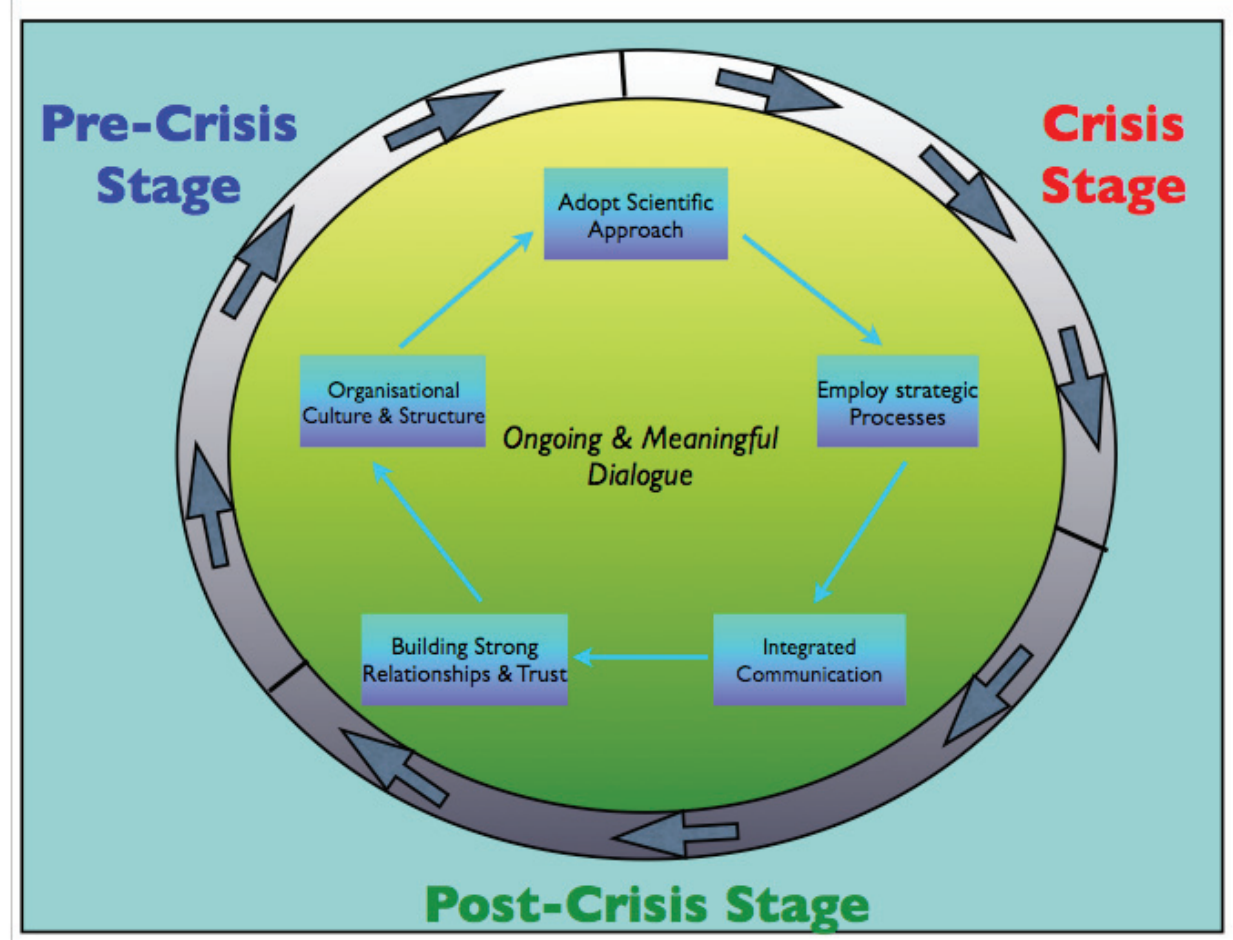

In order to underpin and guide the strategic major emergency communication management process, five of the overarching strategic principles (represented in blue in Figure 5.1) should be applied to each of the three stages of the major emergency life cycle. The five strategic communication principles have no particular precedence within this framework and are represented accordingly in a circular manner. However, a sixth strategic communication principle, ubiquitous and continuous dialogical communication (represented by a green background in Figure 5.1), is afforded a prominent position in relation to the other five overarching strategic principles. Ongoing dialogue should not alone underpin the entire major emergency communication process, but also provide a basis for how communication should be approached when considering the other five strategic principles in turn. 
It is important that each of the six strategic principles is implemented early in the preparation and planning process and should be adopted consistently throughout all three stages of the major emergency life cycle. It is essential that all of the tactical communication functions during a major emergency situation are guided meaningfully by these principles, in as much as is realistically possible. However, the degree to which these strategic principles will be observed is likely to depend on the stage within the major emergency life cycle and the amount of time available. It is extremely important that those responsible for decision-making at the highest level in the lead organisation, are favourably disposed to the adoption of all six strategic communication principles. This may require a considerable amount of persuasive action by communication practitioners in the MECIT, in collaboration with senior management of the lead response organisation.

\subsubsection{Structural and cultural factors}

Section 4.4.1 earlier described the importance of considering structural and cultural factors when developing and implementing a strategic communication management process. This may be considered from two distinct perspectives; firstly from that of the internal culture and structure of the organisation, and secondly, from that of the cultural environment within which the strategy is developed and subsequently implemented. Both of these perspectives are equally important when considering the development of a major emergency communication strategy. As mentioned earlier in Section 4.2.4, the organisational communication strategist needs to consider a 'cultural web' which can potentially result in the acceptance or rejection of the developed strategy. Likewise, the major emergency communication practitioner needs to consider cultural factors such as power structures, control systems and organisational routines.

In the countries examined during this research for this dissertation, major emergency planning, preparation and decision-making are the responsibility of public service organisations that are part of, or closely aligned to, government departments - or that report directly to government. By their nature, public service response organisations generally have hierarchical, closed structures, with clear protocols regarding interaction with the media, stakeholders and the public. In effect, communication 
within these organisations is most likely to move from top to bottom. Additionally, according to ILGRA (1998: 4), many governments often consider the communication function as a mere 'bolt on', where the process is 'institutionalised... and the art of listening is seldom applied'. This can result in communication practices that are overly bureaucratic and restrictive, as evidenced within many post-disaster reports produced by leading countries over the past ten years. These reports are replete with references to communication difficulties that have arisen due to bureaucratic 'redtape', hierarchical communication flows, and communication practitioners being forced to implement the decisions of others. Darrell (2003: 17) agrees and states:

In times of major emergencies, the usual hierarchical decision-making models are often unable to function, and that time-consuming normal bureaucratic systems may need to be simplified.

For example, the US government accepts that the response to Hurricane Katrina was inadequate and cites 'failures within FEMA's architecture and culture' (Townsend 2006: 12). Townsend emphasises the necessity for the federal government 'to transform the way it does business... and to eliminate much of the extraordinary red tape' that caused significant delays in its response (ibid: 70). Due to such 'red tape', FEMA refused to allow evacuation planes to take off without an air marshal on board, thereby delaying the delivery of much needed supplies and causing considerable suffering to the victims of the floods (Sandman 2005). Similarly, Heath (1995: 13) describes how the Japanese government's 'step-by-step, consensus led, group-think process' together with a 'strong face-saving culture' resulted in a very poor response following the 1995 Kobe earthquake in that country. Closer to home, despite its stated commitment to open flows of information in much of its official documentation, the UK government has been accused of being overly secretive in its management of avian influenza. An editorial in the Sunday Telegraph stated:

A culture of secrecy is a serious mistake in this area: it has no national security justification, only that of saving the government from embarrassment. Secrecy generates suspicion and mistrust and can contribute to panic. No government policy to combat an epidemic can succeed without the public's cooperation. That cooperation requires the public to trust the government. Trust is a commodity that is easily destroyed by secrecy (Bird Flu 2006: 20). 
Much of the major emergency literature examined as part of this research supports the premise that an overly bureaucratic and rigid structure within the response organisation can impede the development and implementation of an effective communication strategy. As discussed earlier in Sections 4.3.2 and 4.4.7, when information does not flow horizontally and in two directions throughout the organisation, it can often hinder the adoption of a strategic communication management approach and the smooth two-way flow of dialogue with stakeholders and publics. Contemporary theorists, such as NRC (2004: 8) and Darrell (2003: 17), emphasise placing communication close to the highest level of decision-making, thereby ensuring that communication is considered at every step of the planning process. In order to ensure that all elements of the strategic communication approach are fully embraced, senior decision-makers at the highest level must fully understand and appreciate the value that the process can bring to the overall major emergency effort and provide the necessary resources required to support it. Theorists such as Reynolds (2002) and Nordlund (1994), discussed earlier in Section 4.5, highlight that no operational plan should be developed without a communication element attached. Consequently, communication is no longer considered to be an afterthought or 'bolton', as described above. In keeping with Marra's (1998: 8) opinion, if this acceptance of communication is pushed downwards throughout the entire major emergency response structure, any fears of breaking with traditional top-down and closed forms of communication can be reduced.

It will be shown later in Section 9.4.3 that countries considered leaders in the field of major emergency communication believe that their response architectures are not overly bureaucratic, that their respective communication functions are close to the centre of decision-making, and that information flows both horizontally and in a twoway direction within their own response organisations. This indicates that the communication structures adopted by these countries are evolving. However, there are sufficient examples, some of which were mentioned above, to indicate that problems still exist and that major emergency communication structures and flows within these countries remain far from perfect. Consequently, the model requires ongoing further rigorous testing, review and updating before it can be deemed optimal. 
The culture of the organisation can also impact on the development of a major emergency communication strategy. Marra (1998: 8) describes how many crisis communication programmes are 'destined to fail' because the approach 'contradicts the dominant and accepted communication philosophies adopted by the organisation' In other words, a great major emergency communication strategy is unlikely to work 'if it's not the way we do things around here' (ibid.) - this response being otherwise described as a historicist approach. Shaw (2005:14) identifies a number of reasons why senior management may not support the strategic major emergency communication process and may adopt a historicist approach instead. These include time constraints, a lack of exposure to international trends, 'groupthink', fear of taking responsibility for the outcomes of the communication process, and a lack of personal communication knowledge or experience. As will be seen later in Section 9.3, countries considered leaders in the field of major emergency communication constantly strive to improve their approach to major emergency communication, which is reflected in their strategy documents and protocols. Consequently, major emergency communication management in these countries is considered a living process and any historicist approach is unlikely to be effective.

As discussed in Sections 3.4.3 and 4.4.1, any strategy must be considered within the context of the cultural environment which the organisation operates and the strategy will be delivered. This also applies to a major emergency communication strategy. Because of cultural factors, people are likely to perceive risk and life-threatening situations in a variety of ways. Additionally, people of varying cultural backgrounds are likely to receive and comprehend identical information in many different ways. Consequently, despite a noticeable convergence of major emergency communication theory throughout the international literature and guidance documents, as evidenced in Chapters 4 and 5 and also in Section 9.3, theorists such as Smiramesh, White, Dozier and Frosdick emphasise the importance of being prepared to tailor the communication strategy in order to suit the audience to which it is directed. Consequently, major emergency communication practitioners must avoid slavishly adopting strategic communication protocols and practices that are deemed bestpractice and instead develop strategies that best suit the culture within which the communication process will be delivered. 


\subsubsection{Ongoing and meaningful dialogue}

Fischhoff (1995: 138) describes in Chapter 3 how communication with publics has evolved since the mid-1970s from a crude one-way process of merely providing information, to a more inclusive and dialogical approach. As mentioned earlier in Section 3.7.4, in this contemporary approach to communication, open flows of information exist between the lead response organisation and its publics, information is shared, and publics are treated as partners and meaningfully engaged with in relation to major emergency decisions that are likely to affect them. Engaging with publics such as this equates to the 'relational approach' to communication management, described earlier in Section 3.6.2. This contemporary relational approach is also supported by Salter's model that describes a more holistic view of major emergency management, presented earlier in Figure 2.1. The utilisation of ongoing and meaningful dialogue with publics, as discussed Section 4.4 .7 provides the communication practitioner with greater possibilities of enhancing communication flows to key publics during a major emergency situation by improving levels of openness, transparency, trust and credibility. Consequently, the dialogical approach to communication should be carefully considered by the communication practitioner prior to undertaking a strategic major emergency communication process.

Sandman (2005) describes this process as 'relaxing the bureaucracy... and letting the amateurs in'. Hughes and Henry (2003: 25) consider the involvement of 'nonexperts' as 'the creation of a bridgehead that connects emergency services and the wider community'. Fahey (2003: 12) categorises the use of 'non-experts' or 'amateurs' as a form of 'social capital' and describes how it helps to build 'strong and resilient societies'. Lasker (2004: 1) emphasises that the public 'must be involved directly' in planning and preparation for serious terrorism related incidents, in order for plans to be effective.

The benefits of opening lines of communication with publics and involving 'nonexperts' as partners in the decision-making and planning process for important major emergency decisions are many and have been discussed in some detail by theorists such as Fischhoff (1995: 138), Stern and Fineberg (1996: 3), Kinney and Leschine 
(2002: 84), Rowe and Wright (2001: 342), and Coles and Buckle, cited in Norman, Stuart-Black and Coles, (2006: 92). While not specifically related to major emergency situations, Grunig, Grunig and Dozier's (2002: 145) model of strategic communication management, described earlier in Section 4.3, highlights the importance of an 'interconnectedness' between management and publics, involving a meaningful two-way flow of communication, in order to improve relationships and to achieve organisational goals. However, many contemporary theorists apply Grunig et al's principles to the major emergency communication context. The advantages of the process include: creating an environment of improved awareness and comprehension of important major emergency issues amongst the public (Bowman 2000: 5.1); tapping into community know-how and experience thereby enhancing the overall knowledge-base; ascertaining essential information such as media consumption habits, current states of knowledge and preferred channels of communication (Cottrell 2005: 27); ensuring equity of access to emergency services, especially for marginalised groups (Hocke and O'Brien 2003: 63); reducing uncertainty by ensuring that complicated information can be imparted and received in a format that is understandable to diverse publics (Palenchar and Heath 2002: 131; NRC 2004: 15; Nohrstedt 2000: 140); making decisions more acceptable to publics through a heightened perception of balance, trust, fairness and transparency in the communication process (Stern and Fineberg 1996: 81); and reducing levels of potential 'outrage' (Wilcox, Ault and Agee 1997: 54; Sandman 2005).

Failure to open such lines of communication may result in practical problems that could otherwise be avoided. For example, in the aftermath of the 1986 Chernobyl nuclear disaster in Ukraine, the UK government mistakenly purchased large consignments of Argentinean straw rather than hay as a substitute principal fodder for highland sheep (sheep do not eat straw) (Irwin and Wynne 1996: 36). Additionally, poultry farmers in the UK have described government instructional messages concerning avian influenza as 'confusing' and 'full of ridiculous jargon'. They also describe how a lack of consultation has resulted in poultry farmers receiving instructions that are 'totally unfeasible', such as entirely covering large poultry farms with wire netting (Harrison and Copping 2006: 13). 
When it comes to involving the public in the management of risk issues, including major emergency situations, many varied opinions abound (Fahey 2003: 12). A school of thought exists that involving 'non-experts' in decisions related to hazardous situations can result in confusion (Fordham 1998/99: 31) or can cause needless delays or conflict (Stern and Fineberg 1996: 135). Rothstein, cited in Ball, (2006: 6) warns that 'participative reforms', such as involving publics in important risk decisionmaking, 'often promise more than they can deliver'. According to Kweit and Kweit (1984: 32):

The public lack the technical expertise, are unfamiliar with bureaucratic routines and are emotionally involved in issues rather than being detached and rational. Citizens are outside the hierarchy and therefore hard to control. As a consequence, participation may increase the time needed to reach decisions as well as the level of conflict.

Ball recognises advantages to strengthening inclusiveness by opening lines of communication and engaging with stakeholders and the public in decision-making. However, he states that it is 'a much more complex task than has often been acknowledged' and is 'wide open to manipulation and exploitation' (2006: 9).

While there are clear advantages to be gained from adopting the process of dialogical communication for major emergency situations, its acceptance is not universal and many theorists highlight the fact that the paradigm is not without its inherent difficulties. In the first instance, the resources and effort required in order to undertake a 'broad-based' dialogical approach are likely to be substantial. This raises the question of whether or not these resources would be better utilised elsewhere, particularly while resources are likely to be scarce during the crisis and post-crisis stages. Consequently, response organisations need to consider a cost-benefit analysis of the process, taking into account how much time and effort they spend, by how much communication decisions are likely to improve, and the net benefits for stakeholders and publics. While the dialogical approach is far more democratic than using mere one-way propaganda, or two-way asymmetrical persuasive techniques, these latter techniques may be much more cost-effective, particularly where an apathetic public is involved. While countries examined as part of this research have espoused the principles of dialogical communication over the past decade, FEMA provided less than satisfactory responses to recent major emergencies such as 
Hurricane Katrina and the UK government were criticised for many communication aspects of official responses to the London bombings of July 2005.

Nevertheless, adopting a broad-based dialogical approach provides many advantages to the major emergency communication process, particularly the 'non-expert' information and advice that can guide the development of protocols as was described by Lasker. Bowman (2000: 6) emphasises that engaging stakeholders in dialogue in relation to major emergency communication planning and response will not of itself achieve a great deal. However, as part of a wider range of communication efforts, it can help to educate, inform, and engage the public, which may ultimately provide an improved public emergency response. Significantly, as mentioned earlier in Section 3.7.4, meaningful engagement with stakeholders and publics should not exclude the vital role of, and crucial information that can be provided by, the scientific community. As the 'dialogical' communication process is an evolving field of endeavour, a careful and rigorous evaluation needs to take place before the approach is considered best-practice. It is not beyond reason to believe that the 'dialogical' approach could prove to be cumbersome and inefficient, and in the tradition of communication theory, as discussed earlier in Chapter 3, be surpassed by a new paradigm. Additionally, as described in Section 5.3.2 earlier, any broad-based dialogical strategy needs to be considered in the context of the cultural environment within which it is developed and implemented. While one approach may work extremely well in one country, it may prove less effective in others.

\subsubsection{Adopting a scientific approach to communication}

Approaching communication from a scientific perspective is important in order to ensure that the right messages are getting to the right people in the most efficient manner. This approach is equally important when considering a major emergency communication strategy. A scientific basis is central to the strategy formulation process, as described earlier in De Wit and Meyer's 'diagnosing' phase in Figure 4.1. As discussed in Section 4.5, Reynolds (2002), UK Resilience (2005) and Nordlund (1994) in their models of major emergency communication place considerable emphasis on adopting a scientific approach to communication. A scientific approach involves a number of key elements. 


\subsubsection{Sound knowledge base}

Theorists such as Grant (2008), Dranove and Marcianos (2005) and Grunig (1992) (Section 4.4.8) suggest that possessing formal education is a very useful means of building a knowledge base, thereby allowing practitioners to adopt a more scientific approach to communication management. According to Reynolds (2002: 38), the need for competence and expertise requires a major emergency communication team to demonstrate 'levels of formal professional education, previous experience, and training'. Coombs (1999: 150) agrees and goes further noting that '...crisis communication teams should be equipped for success...equipment for success includes possessing essential knowledge and skills'.

\section{Formal education and training}

One of the best means of improving the response organisation's knowledge base and scientific approach is through formal strategic communication-related education and training. Price (1989: 124) concurs and states:

A major element helping to determine the credibility of risk and crisis messages is the perceived competence of the individual or organisation managing the major emergency...many government agencies do not have sufficient staff with the necessary professional educational competencies.

Darrell (2003: 22) emphasises how theoretical knowledge combined with experience can 'build the credibility of the individuals managing communication' during a major emergency. Iwan (1999: 24) describes how the impact of a number of natural disasters, such as a tsunami in Japan in 1994 and a volcanic eruption in Alaska in 1992, could have been greatly reduced had emergency responders received the benefit of formal communication education and support from academic institutions. Formal communication education courses are best considered during the pre-crisis stage of a major emergency. However, less-formalised communication training and education can prove extremely useful during the crisis stage of a major emergency. For example, Grady (2005: 11) describes how doctors and nurses with experience in Ebola outbreaks, provided information and communication seminars for health workers in Angola following an outbreak of Marburg virus in the Luanda region in 2005. 
One element of education and training that must not be overlooked by the major emergency communication practitioner is that of providing education and skills to local communities in order that they can protect themselves. This has been discussed in Section 2.4.1.2.2. While many barriers are likely to exist to such a programme, including community apathy and competing interests such as sport, family life and work, if successfully implemented it is likely to provide a useful 'force multiplier' to the emergency services. This will be dealt with later in Section 6.4.2.6.

\section{Experience}

In addition to formal communication education and knowledge, ILGRA (1998: 18) Reynolds (2002: 39) emphasise the necessity for members of the MECIT to have requisite experience in handling all aspects of the communication function for all stages of an emergency situation. Secondment programmes, particularly to international relief agencies, provide a useful means for MECIT members to receive 'hands-on' major emergency communication experience. In the absence of real 'hands-on' experience, some communication practical knowledge can be gained through the use of realistic scenario-based exercises. The benefit of exercises is discussed in the next chapter.

As discussed in Chapter 3, many of the communication concepts described within this framework are derived from work undertaken by academics from the 1920s onwards. A theoretical derivation of the concepts of scholars such as Lippmann, Lasswell, Lazarsfeld, Hovland and Schramm can greatly benefit the work of major emergency communication practitioners, particularly in relation to understanding the attitudes and likely behaviours of interested and affected publics. Additionally, the work of these pioneering communication theorists creates an awareness for contemporary major emergency communication practitioners of the many benefits that can accrue from taking the time and effort to engage in research. This in turn can bring fresh communication concepts, ideas and approaches to the organisation. However, a careful balance must be achieved between engaging in formal education, practical learning and experience. In accordance with Reynolds (2002: 31) and Darrell's (2003: 22) view, the competent communication practitioner should possess both theoretical knowledge and experience. Consequently, major emergency education should 
provide merely the foundation for communication practitioners. This theoretical foundation then requires testing, contextualising and enhancement through real-life or simulated experience.

\subsubsection{Ongoing communication research}

The conduct of ongoing and iterative communication research, throughout all three stages of the major emergency life cycle, is another important element of the scientific approach to strategic major emergency communication management. As previously discussed in Section 4.4.4, any strategic communication programme that is undertaken without research is likely to be flawed. Indeed, De Wit and Meyer's (2004: 106) model, described in Section 4.2.2, identifies research as a critical function among the strategy formation activities. Additionally, Grunig's (1992: 124), normative model of strategic communication management, described in Section 4.3.2, includes continuous research as an important function. Darrell (2003: 22) emphasises how it is essential that the response agency continues to engage in constant communication research. In the initial stages of the major emergency communication research process, Austin and Pinkleton (2000: 49) discuss the value of 'formative research'. This type of research provides the MECIT with essential data to help it define the communication problem and to guide the strategic communication planning process. In this regard, formative research can be used to identify any potential hazards in the environment; identify the many stakeholders, publics and audiences that may be affected by a major emergency; determine their concerns, issues and needs; understand how different publics perceive risk and hazards in their environment; determine how different publics are likely to react to major emergency risk and crisis messages; discover any gaps in the state of knowledge; and determine how best to reach affected publics.

In addition to formative research, Grunig, Grunig and Dozier (2002: 209) identify 'programme research', which provides useful information to guide the implementation of the major emergency communication programme. Researching and testing the efficacy of communication messages and channels prior to a largescale major emergency public information effort is an example of programme research. In order to evaluate the success of a particular communication programme, 
based on the achievement of stated objectives, Dozier, Grunig and Grunig (1995: 34) discusses the value of 'summative (or evaluation) research'. Evaluation research is discussed further in the next section. Darrell (2003: 22) highlights the importance of providing adequate training to guarantee that all communication research is conducted strategically, and that sufficient resources are provided so that this important research function is carried out during all stages.

While an evidence base exists that supports major emergency communication research, it is very time-consuming and it is likely in practice that time and resources will be extremely scarce for such programmes. During the pre-crisis stage, the lead response organisation is likely to have ample time to conduct extensive communication research. However, pressures generated in the immediate aftermath of a major emergency are likely to limit seriously the scope of any research. While major emergency organisations might be convinced to engage in once-off research, such as the Millward and Brown IMS (Department of Defence 2005(a)) survey of attitudes in Ireland, it may prove difficult to convince decision-makers to pursue such programmes on an ongoing basis. This highlights, once again, the importance of having the senior communication practitioner close to the centre of decision-making. Despite the obstacles to engaging in strategic communication research, the benefits can far outweigh the costs, particulary for understanding the breakdown of key publics, knowing their issues, needs and concerns, how best to reach them, and the type of message that will optimise a desired response that may ultimately save lives.

\subsubsection{Ongoing communication evaluation}

A final element in the scientific approach to strategic major emergency communication management involves the MECIT engaging in ongoing communication evaluation. De Wit and Meyer (2004: 106) (see Section 4.2.2) include the evaluation of implemented actions as an essential part of strategy formation. Additionally, Grunig (1992: 124) and Nordlund (1994: 40), in their models, emphasise the importance of carefully evaluating communication programmes on an ongoing basis as an important strategic function. 
Reynolds (2002: 10) notes the importance of ' ... evaluating the major emergency communication plan performance, documenting lessons learned, and determining specific action to improve crisis systems or the plan'. Coombs (1999: 136) discusses how strategic evaluation should not be conducted merely at the end of a communication programme, but should be carried out 'regularly and systematically throughout all stages'. This ensures that sub-optimal communication programmes or messages can be reviewed and revised to achieve greatest effect. MOR (2002: 58) describes how evaluation, or review, ensures that the major emergency communication programme is 'focused and controlled, thereby ensuring continual progress towards the desired outcome'. Price (1989: 163) states that communication evaluation, when coupled to an effective feedback mechanism, '...is a necessary step in ensuring improvement in the competence of an organisation's risk and crisis communication programme'.

Pieczka (2000: 214) also discusses the importance of 'implementation evaluation' that assesses the extent to which the major emergency communication plan, when triggered, is implemented effectively. Conducting integrated major emergency exercises helps to evaluate the success of the implementation process of communication strategies and plans. In the UK, during the 'foot-and-mouth' outbreak, the use of government direct mail shots and the Department of Agriculture website proved, in hindsight, to be ineffective. An 'implementation evaluation' of the communication process could have highlighted that the information disseminated through both of those channels was consistently out-of-date and of no significant value to the recipient (Anderson 2002: 6). Hon (1998: 106) emphasises the importance of 'impact evaluation', which assesses the consequential impact of the major emergency major emergency communication plan or programme. For example, Handmer (1990:139) describes the importance of evaluating the impact of flood warnings in the Thames Basin and how this evaluation helped to improve the systems of warning communication for the public in that area.

While it is evident from the research that there is clear support for engaging in ongoing communication evaluation, in practice it is likely to prove difficult during the crisis and early post-crisis stages, due to inevitable demands on time and human resources. Consequently, the communication practitioner will have to conduct a 
rigorous cost-benefit analysis in order to determine the amount of resources and effort required in the communication evaluation process. As with communication research, practitioners may have to demonstrate the benefits to operational commanders of engaging in the evaluation process, such as testing the efficacy of communication programmes or messages or channels, in order determine which communication programmes are not working and to refocus the effort. Often this is difficult to achieve as communication managers may be reticent to acknowledge that particular programmes are not working - particularly if significant time, cost and effort have been expended up to that time. Through a judicious use of basic tools such as awareness, attitude- and opinion-surveys, in-depth interviews, content analyses, inquiry feedback, and impression counts, a communication evaluation should not have to take up too much time or too many resources.

\subsubsection{Employ strategic processes}

An important element of the strategic major emergency communication framework involves consideration of the actual processes adopted by the communication practitioner, which by their nature need to be strategic as distinct from tactical. As discussed in Section 4.2.2, many management theorists such as Grant (2008), Hill and Jones (2001), and Mintzberg and Quinn (1991), describe the importance of organisations employing strategic processes in order to allow them to co-exist, interact and survive with their publics, particularly when the environment is uncertain. These concepts have been employed and supported by many communication management theorists such as Grunig (1992: 4), Plowman (2005: 132) and Cutlip, Centre and Broom (2000: 6), as described earlier in Section 4.3.

The major emergency environment offers additional challenges and involves dealing with a number of simultaneous complex communication problems in extremely pressurised conditions. Many contemporary theorists, such as Reynolds (2002), Nordlund (1994) and UK Resilience (2005), emphasise the importance of employing such strategic management processes while planning, preparing, and implementing the major emergency communication function. As described earlier in Section 4.3.1, by engaging in a non-strategic (tactical) approach to communication management, the communication practitioner is likely to ignore the detailed, integrated, longer-term 
planning considerations required for effective communication management. This can result in the MECIT operating without adequate direction or a thorough understanding of the situation or sufficient resources or information - particularly when time is of the essence. Consequently, as part of the proposed framework, the communication practitioner should give careful consideration to the following strategic elements.

\subsubsection{Management-by-objectives $(\mathrm{MBO})$}

The benefits of $\mathrm{MBO}$ as a strategic process for effective organisational strategy and communication management, have been clearly outlined in Section 4.4.2, and are supported by theorists such as Hill and Jones (2001: 429), Seitel (2004: 81), and Grunig (1992: 11). The majority of major emergency literature, including that by Nordlund (1994: 17), UK Resilience (2005: 27) and Reynolds (2002: 20), examined as part of this research, provides evidence that setting clear communication goals and objectives is increasingly considered an important element of the major emergency communication process. MBO should not be an overly time-consuming process, but is an important stage of the strategic process in order to guide the entire major emergency communication effort and to ensure that it fully supports the overall operation.

One of the most significant advantages of the MBO process is that the MECIT will have a clearer understanding of what needs to be achieved in the short-, medium-, and long-term. Darrell (2003: 24) reinforces this point emphasising that the communication mission statement allows everyone involved in the response effort to clearly understand their roles, goals and objectives. Without such clarity responders are likely to operate at cross purposes. In this regard, Smith (2005: 72) states that the major emergency communication goals and objectives should be '...clear and measurable statements based on the goals of the organisation, and written in such a way as to point the way toward a particular level of awareness, acceptance or action.' Nordlund (1994: 17) states that communication goals and objectives should be developed for all stages of a major emergency life cycle. While the list of communication goals and objectives is likely to be extensive and diverse, the series of communication tactical steps outlined in the proposed frameworks in Chapters 6, 7, 
and 8 provides the lead response agency with a useful starting point for developing this process. Each individual communication goal and objective should be supported by a clear action plan that details clearly the person responsible for completing the action and the timeframe within which it is to be completed. In as much as is possible, this management-by-objectives process should be developed during the precrisis stage when time and resource pressures are less demanding. However, protocols should be established to allow a more condensed process of goal and objective setting for the communication function during the chaotic crisis and post-crisis stages of a major emergency. All goals and objectives need to be evaluated and reviewed on an ongoing basis in order to assess the likelihood of them being achieved.

Significantly, in keeping with the views of UK Resilience (2005: 28) and Nordlund (1994: 33), the MBO process makes senior major emergency communication practitioners more accountable for their decisions and allows for a greater coordination of effort. As communication success is often difficult to measure (unlike many operational functions), setting clear, measurable major emergency communication goals and objectives creates a clearer focus for communication managers and provides a more transparent quantum of success or failure for their efforts. However, as with many of the strategic communication elements discussed as part of this framework, the MBO process in relation to major emergency communication is a relatively recent phenomenon. Consequently, the advantages need to be closely assessed in order to determine its usefulness and in particular its role in improving the quality of communication provided to stakeholders and the public.

\subsubsection{Close alignment of goals}

Closely associated with the MBO process, and central to the ethos of the strategic process, is the alignment of goals to a common purpose. Communication theorists such as Hon (1998: 105) and Grunig (1992: 262), discussed earlier in Section 4.4.2, highlight that a fully integrated and cohesive major emergency communication strategy requires that all communication goals and objectives are closely aligned with those of the overall strategic operational plan of the lead response organisation in a 'tight-fitting nexus' (Temple 2002: 33). If this is not achieved the operational and 
communication function is likely to be at cross-purposes and instead of providing a supportive role, the communication function could prove counter-productive to the response and relief effort during a major emergency. Placing the senior communication practitioner close to the centre of decision-making can assist in the alignment process.

The most obvious benefit in the alignment of goals is when there is a multitude of diverse response organisations involved in a complex major emergency situation. The alignment process can assist the overall commander in coordinating and optimising the operational and communication efforts of organisations such as government departments, local authorities and 'blue-light' services (including police, fire and ambulance, armed forces, community organisations, and aid agencies). Additionally, such alignment of operational and communication goals across diverse organisations can assist in the avoidance of duplication of effort and in the optimisation of resources. As regards communication management particularly, the alignment process can assist in ensuring a consistent approach in relation to levels of openness and transparency. Additionally, conflicting information from different response organisations in the dissemination of key messages can be avoided.

However, the cross-agency coordination of communication goals and objectives must be sustained throughout all stages of a major emergency life cycle. EMA (2003: 2), discussing the 'lessons learnt' from the 9/11 terrorist attacks in New York, emphasises:

The most important lesson was undoubtedly that cooperation and goodwill has to exist between all levels of the response team...interoperability, shared goals, standardised communication systems, and information sharing are key areas that can be enhanced to enable a coordinated multiagency approach.

Achieving such cooperation is likely to prove a demanding task, requiring response organisations to overcome issues such as internal politics, power struggles and different levels of skill and training. Yet the benefits of such cooperation to the overall response effort are likely to be immense. The provision of clear protocols and guidelines, coupled with ongoing inter-agency training and exercises are likely to assist greatly in this process. A well-established and controlled communication and 
information management centre (CIMC) can also assist in achieving the coordination of inter-agency communication and operational goals and objectives.

\subsubsection{Engage in strategic communication planning}

In Section 4.4.3, the importance of engaging in strategic planning as part of the strategic management process was discussed and supported by the arguments of theorists such as De Wit and Meyer (2004) and Finlay (2000). Strategic planning is increasingly utilised by communication practitioners in order to deal with complex situations. In Section 4.4.3, the benefits of engaging in strategic communication planning were discussed. These include providing an impetus for detailed analysis and strategic thought throughout the entire communication process, providing a mechanism by means of which an optimal course of action can be chosen, and generating a platform for increased levels of proactive communication. The strategic planning process can assist the communication practitioner throughout all stages of a major emergency situation and should be a central element of the strategic process.

In accordance with Grant (2008: 21), the strategic communication planning process allows for the major emergency communication and information team (MECIT) to define clearly its communication problems, identify suitable approaches for addressing these problems, determine responsibility for achieving stated goals and identify resources required over the planned time period. As acknowledged by UK Resilience (2005: 60), Nordlund (1994: 8) and Daugherty (2003: 21) such strategic communication planning allows the MECIT to provide systematically a comprehensive communication response for all stages of a major emergency situation. Without such planning, the communication response is likely to be delayed considerably once a major emergency occurs and confusion is likely to exist as regards roles and responsibilities. Many communication theorists such as Bernstein (1987: 13), Higgins (1979: 9) and Fearn-Banks (1996: 24) have identified advantages in adopting a strategic approach to major emergency communication planning, including having the ability to anticipate problems, respond effectively, contact key personnel, build useful networks, prepare key generic messages and information facts sheets and reduce the number of unknowns overall. Additionally, bringing together key communication personnel as part of the strategic planning process is a means of 
considering many diverse opinions and ideas, thereby facilitating a better communication response. The process also allows key communication personnel to work together prior to a major emergency.

As described earlier in Section 4.2.1, strategic planning is an essential element of the overall strategic management process. In this regard, the MECIT's communication planning process needs to be fully integrated into the lead response organisation's overall operational plan. The output from the strategic planning process is the production of a comprehensive major emergency response communication plan (MERCP). The development of this plan is discussed in greater detail in the next chapter. While most of the communication planning is best conducted during the precrisis stage of a major emergency, it is essential that the process should continue throughout the crisis and post-crisis stages (Reynolds 2002: 69). This is particularly important for the development of new communication plans and the possible modification and updating of existing MERCPs. This is considered essential, as the strategic planning process is unlikely to be able to deal with all eventualities that are likely to arise due to the unpredictability of major emergency situations.

While the advantages of the strategic communication planning process have been clearly outlined above, practitioners need to ensure that they avoid intransigent adherence to pre-prepared major emergency communication plans, as this is likely to hamper the response effort. The major emergency environment is such that practitioners need to be able to deal with rapidly changing scenarios. Relying wholly on the output of strategic communication planning can result in a lack of flexibility when it comes to dealing effectively with rapidly changing situations. However, given an appropriate model of strategic major emergency communication planning, together with sufficient training and exercises, communication practitioners should be able to respond appropriately to evolving situations. Heath (2001: 487) proposes adopting 'anticipatory models' of strategic major emergency communication planning, including approaches such as 'scenario planning'. Ringland, (1998: 2) describes such approaches as a series of possible scenarios and a number of different courses of action and solutions, using 'war-gaming' and 'brainstorming' techniques, in order to overcome identified problems. 


\subsubsection{Engage in strategic decision-making}

An important element of the strategic communication management process, already mentioned in Chapter 4, is choosing optimum courses of action through strategic decision-making. According to ILGRA (1998: 15):

Crisis conditions demonstrate more than any others the need for welldeveloped systems, procedures and technology in order to be able to make consensual decisions and to communicate quickly, consistently and reliably with large audiences.

Nordlund (1994: 43) agrees, noting how ' ...poorly conceived and implemented communication decisions can result in a greater chance of death or destruction in times of a major emergency'. In some situations, poor communication can actually create harm. Strategic decision-making is a central function of strategic communication management and includes two separate elements which are reflected in De Wit and Meyer's (2004: 106) model described earlier in Section 4.2.2, as 'option generation' and 'option selection'. It is at the decision-making stage that leadership adds value to the entire communication function. Approaching the communication decision-making process from a tactical or 'seat-of-the-pants' perspective is likely to result in all available information not being utilised and all courses-of-action not being considered. Consequently, there are clear advantages in adopting a strategic approach to communication decision-making, that involves a series of steps including, deciding what the problem is; selecting a wide base of participants to the decision-making process; establishing clear protocols for actions if decisions cannot be reached; making all of the gathered information intelligible to all decision participants; discussion, negotiation and bargaining by 'expert' and 'nonexpert' participants; and selecting suitable courses of action (Stern and Fineberg 1996: 143). This decision can then be implemented and the impact evaluated in order to determine the efficacy of both the decision-making process, and the impact of the major emergency communication decision on the intended recipients (Comfort 1988: $185)$.

While the traditional hierarchical decision-making model is a faster process than this proposed dialogical approach, the benefits of including both 'expert' and 'non-expert' opinion provide many advantages, as described earlier in Section 5.3.2. A balance 
needs to be reached as regards the amount of both 'expert' and 'non-expert' participation in the process, particularly taking time constraints into account. Additionally, as part of the process, clear protocols must exist in order to deal with situations when decisions cannot be reached. Otherwise, in a fast-moving major emergency situation, important decisions may never be arrived at. Consequently, it may be necessary to compress many of the steps recommended above. Once again, continuous integrated communication training and exercising will help to identify and deal with many of the problems that are likely to arise.

\subsubsection{Integrated communication}

An analysis of the major emergency communication models, described earlier in Section 4.5, including those by Centers for Disease Control (Reynolds 2002: 7) and UK Resilience (2005: 28), highlights the absence of an integrated or joined-up approach towards all of the key communication functions. Each of the models deals with many of the individual communication functions in some level of detail, however, none of them offers a truly integrated and multidimensional approach. The strategic framework proposed in this chapter integrates all of the key communication functions, under the control of the lead response organisation's MECIT, including public information management, information management, internal communication, media management, and community outreach and victim liaison.

Each of these key communication functions is a subject of significant research in its own right. However, due to the scope of this dissertation it is impossible to deal with each function in any great detail. Consequently, some of the functions are dealt with in significant detail during this chapter, while other functions, such as public information management, receive more detailed attention throughout the next three chapters. As both the information management and media management functions are particularly important throughout each of the three stages, it is prudent to deal with them in detail within this chapter. 


\subsubsection{Public information management}

The public information management role involves researching, developing and testing major emergency risk and crisis messages, and information, and disseminating this to stakeholders, different publics and to the public at large. The purpose of this important role is to inform and educate these people and to adequately prepare them to deal with, and respond to, major emergency situations, thereby saving lives. This function is viewed by many theorists as being central to the MECIT's role and is equally important in all three stages of a major emergency life cycle. It is therefore appropriate to deal with this subject in considerable detail throughout the next three chapters, particularly Chapter 7.

\subsubsection{Information management}

Closely related to the public information management role, but often a function that is not viewed frequently as a communication manager's role, is that of information management. According to Jacobs (1990: 1332), in a major emergency situation, accurate information 'is a priceless and at times decidedly rare commodity'. A very likely problem faced by the lead response organisation once a major emergency actualises is a dearth of useful information. Cohn (2000: 186) mentions that the period immediately following a major emergency is characterised by 'little information and lots of chaos'. Coombs (1999: 115) agrees and highlights how an 'information void or vacuum' can occur as the crisis situation unfolds due to inadequate information management systems. Tumelty (1990: 16) highlights the poor information flows that existed during the North Sea Piper Alpha oil-rig explosion in 1988, which resulted in the deaths of 167 workers.

The initial problem, which has been reflected in other disasters, was the sheer chaos, confusion and lack of information. The last of these led, almost inevitably, to a welter of rumours moving into the vacuum.

Paradoxically, another problem that may arise for senior decision makers during a major emergency involves a profusion of incoming unfiltered raw data to the emergency operations centre (EOC). This may result in decision-makers spending much valuable time attempting to prioritise and make sense of vast amounts of information. It may also result in the information officers within the MECIT 'cherry- 
picking' conspicuous elements of the data, resulting in important decisions being made based on this unfiltered information which is often compounded by their personal experience and historical case-studies.

Information management cannot be considered as a stand-alone function but must be part of, closely associated with, and guided by the strategic communication function. Consequently, the efficient management of data by communication officers throughout a major emergency situation 'is a priority' (Hodgkinson and Stewart 1991: 109). Bernstein (1987: 21) and Fernandez and Merzer (2003: 99) discuss how the lead response organisation must have the capability to immediately collect, compile, confirm, assess and disseminate 'hard' information as it becomes available following a major emergency in order to provide a sound basis for decision-making and communicating effectively with publics. This is important as 'uncertainty can often run high due to poor information flows' (Bernstein 1987: 21). Despite the intellectual challenge associated with this function, the lead response organisation requires an information management system within the MECIT that is intrinsically simple and fully integrated with the operations function together with other communication functions. Granger (2000: 20) offers a useful model describing four distinct phases of an information management cycle, including information direction, collection, processing and dissemination.

Taking on board communication management lessons learned following Hurricane Katrina, the US government is now in the process of fully integrating each of the key communication functions, including information and media management, within its revised Joint Information Centre structure (NRT 2000: 2-1).

\subsubsection{Internal communication}

An extremely important communication function that is often overlooked in major emergency planning, preparation and response, is the management of internal communication. This important communication function involves the communication of important messages and information to government departments and other response agencies, and critically, to members of response teams and their families. Fernandez and Merzer (2003: 153) emphasise the importance of communicating with 
individual responders and their families. They describe how families of responders would 'prefer to hear any important news from their employer than from the press or through the rumour machine'. Darrell (2003: 18) mentions how effective communication with this critical class of stakeholder is often overlooked. Ganley describes how many on-call fire fighters heard of the 9/11 incident through channels of communication such as television news and phone calls from relatives, rather than through official channels (Ganley 2008). Bernstein (2002: 23) describes how the families of fire fighters, who were killed in the 9/11 attacks, learned of their deaths through the media rather than through formal internal communication channels. Chong (2006: 10) remarks how efficient internal communication systems are particularly essential during health epidemics when medical and nursing personnel, themselves, are extremely vulnerable to becoming victims. Reynolds (2002: 14) stresses the importance of keeping this internal audience informed by such means as electronic communications and personal visits. Fernandez and Merzer (2003: 154) include channels such as teleconferences, toll-free phone lines and written letters.

This extremely important function should be a key role of the MECIT throughout all three stages of the major emergency. This is a relatively easy task in the pre-crisis stage. However, as many major emergency situations occur and pass very quickly, there may not be sufficient time to communicate in any great detail with responders and their families in the crisis stage, aside from operational updates. In the post-crisis stage the internal communication channel must operate efficiently. This subject receives further attention in the next two chapters.

\subsubsection{Media management}

Media management is another critical task that is central to the strategic major emergency communication management function. It is extremely important that the lead response organisation works closely with the media throughout all three stages of a major emergency situation. During such events, the media are often considered a hindrance to the smooth running of the major emergency operation. However, the media can prove to be a very useful communication channel for important information and can significantly influence the public's perception of the response effort. 
According to Susskind and Field (1996: 211), the overriding goals of the media are to inform, educate, entertain, reflect a broad spectrum of views, provide a public forum for 'expert' and 'non-expert' dialogue, and to explain the content of public debate. In addition, Scammell and Semetko (2000: 10) consider the role of the media as an independent 'critical watchdog' - whereby they are tasked with the monitoring and reporting on the actions of public bodies, including response agencies - as important. Modh (2003: 5) considers the role of the media as a 'dedicated provider of information and news' as 'crucial' prior to and following a major emergency. Effective fulfilment of this media role, she states, can help to save lives, alleviate suffering, prevent rumours and reduce uncertainty. Reynolds (2002: 129) concurs and emphasises the important role that the media plays in communicating the nature of a disaster situation, warnings, alarms, and the appropriate actions that citizens should take in order to mitigate morbidity and mortality. Nordlund (1994: 43) adds:

Whenever citizens experience an acute need for information and knowledge, the role the media choose to play becomes crucial.

Berge (1990: 69) emphasises the importance of the media in 'providing useful information to the public in simple everyday language'. Kreps, cited in the National Research Council, (1980: 46) highlights how the provision of accurate media information, such as casualty lists and instructing information, can help to alleviate pressure on emergency help-lines.

At a strategic level, the involvement of the media in major emergencies can result in influencing and setting critical agendas. According to Tierney, cited in Alesch et al., (2004: 170):

The mass media has a tremendous ability to increase or decrease the salience of policy issues both within the public and among influential groups.

\section{Media interest in major emergency situations}

During the pre-crisis stage, when the occurrence of a potentially ruinous situation is remote, the lead response organisation is likely to have to deal with a media that displays little interest in major emergency issues. Consequently, this impacts on any 
attempts by the MECIT to raise public awareness and knowledge about such issues through media channels. According to Auf der Heide (1989: 20), 'disasters are lowprobability events... as such they compete for attention with the priorities of daily living'. Walter (2005: 129) blames this circumstance on journalists' 'commercial imperative to get ratings' - and stories relating to major emergency preparation and planning 'are not likely to sell copy'.

On the other hand, once a major emergency actualises a significant level of media interest can be expected. Howarth agrees with this and states:

Disasters, crises and emergencies can strike suddenly and unexpectedly, anywhere at any time. The causes may be sudden and unpredictable. One factor, however, is certain. The news media will be close behind (Howarth 1999: Foreword).

Reynolds (2002: 129) believes a heightened interest will take place particularly if the negative situation is considered to be 'exotic, catastrophic, or the first of its kind'. Nordlund (1994: 31) concurs and surmises that news media tend to be totally preoccupied with the effects of the 'the big bang', or the actualisation of the major emergency.

In the post-crisis stage, a major emergency often tends to become extended and drawn-out. As a result, the media are likely to lose interest and to look for alternative angles, such as identifying people to blame for allowing the situation to occur in the first instance, inefficiencies manifest in the relief effort, or insensitivity displayed to victims by response organisations.

\section{Challenges for the MECIT}

Unlike thirty years ago, major emergency communicators today are obliged to react more quickly in order to meet the requirements of the media. Theorists, such as Nohrstedt (2000: 150), describe how reportage has evolved as a result of exponential transformations in the media landscape, such as advances in broadcast technology (particularly lightweight cameras and satellite communication systems), the development of 24-hour rolling news, and the proliferation of media outlets. For example, these developments allowed a small number of reporters to gain access to 
and broadcast images of the 2004 Tsunami from the remotest regions of Banda Aceh within a few hours of that major emergency occurring (Walter 2005: 83).

Additionally, the MECIT may have to deal with particular difficulties related to media reportage of major emergency events, including media sensationalism of particular risks, hazards and major emergency situations; the trivialisation and oversimplification of some complex emotional events following major emergencies; considering devastating events as quasi-entertainment rather than news; a tendency to invade the privacy of victims, their families, and response workers. Another significant problem concerning contemporary media reportage is a tendency for editors to focus on speed rather than accuracy. According to Reynolds (2003: 26), ' $90 \%$ of first reports following a major disaster event contain factual errors'. Competition amongst media outlets is a major contributing factor to all these phenomena.

\section{Dealing with the media}

As a result of the many difficulties associated with contemporary media coverage of major emergency situations, described above, it is essential that the MECIT deal with reporters, broadcasters and editors in an open, balanced and regulated manner in order to ensure that any reportage provides important information that can save lives or relieve the suffering of affected publics. However, while this may be of great benefit to the response effort, any regulation of media reportage can impact negatively on the impartiality of their output if not handled appropriately. In the aftermath of the First Gulf War, many media organisations complained of the pressures brought to bear on reporters by the Allied Joint Information Centre. Many reporters believed that they were overly regulated through a form of tacit censorship. Consequently, any attempted use of the media should be approached in the interest of public safety and for important logistical reasons. Response agencies should avoid attempting to regulate media output for reasons other than these.

Both Darrell (2003: 11) and Coombs (1999: 35) emphasise how the media can positively or negatively influence the public's perception and image of how the major emergency is being handled and whether the public considers the response agency to be trustworthy and credible. Darrell emphasises: 
Authorities must assume that the crisis image is as real as the crisis itself and that the substandard handling of the image during a crisis can result in the actual crisis expanding or taking a different form and direction (2003: $11)$.

Consequently, EMA (2003: 3 ) describes how an effective media plan is essential in order to deal with the demands of local, regional, national and international media to ensure the provision of accurate, timely and essential information. According to AKNZ and EU (2001: 50), it is important to have experienced media specialists within the MECIT 'who really know how to deal with the press'.

\section{The communication and information management centre (CIMC)}

The NRT (2000: 1-1) describes the necessity for a 'communication hub', where multiple response agencies and organisations can efficiently manage information flows from the major emergency flash point, to a centre where it can be comprehensively analysed and then disseminated to all response agencies, the media, and interested and affected publics, in a controlled manner. For the purpose of this dissertation, this location is termed a 'communication and information management centre' (CIMC). The CIMC should be established and coordinated by the MECIT of the lead response organisation once a major emergency actualises. In this centre, practitioners for all of the key communication functions (described throughout Section 5.3.5) from the many diverse response organisations ' ...can work together to ensure the timely, accurate, easy-to-understand, and consistent dissemination of official information between themselves, to the public and the media' (Ridge 2004(a): 29).

The CIMC is an important focal point for building, gaining and maintaining public trust and confidence, facilitating the control of rumours, analysing public perceptions to the response effort and disseminated messages, and maintaining records and information thereby improving the ability to carry out post-incident assessments (NRT 2000: 1-1 and 1-4). A properly established and run CIMC ensures an optimum distribution of key resources to individual MECITs, such as communication research and evaluation support, crucial logistics and adequate communication information and technology (CIT) facilities. 
The CIMC should be co-located with the major emergency operations centre (EOC) to allow a smooth transfer of information between the operations and communication functions. Additionally, the CIMC should have available representatives from each operational response region, each response agency, appropriate private-sector industry and service utilities, critical transport organisations, government departments, and non-governmental organisations. Ridge (2004(a): 30) and NRT (2000: 1-2) describe how a single CIMC location is preferable during a major emergency so that resources can be better managed and duplication of effort is minimised. However, Ridge (2004(a): 30) also emphasises that multiple CIMCs may be necessary to deal with a complex situation spanning a wide geographical area. In some situations this involves coordination across national boundaries, which often requires great diplomacy. For example, following the 2005 earthquake in Kashmir, emergency communication practitioners in India and Pakistan were obliged to coordinate cross-border communication and information flows (Mountain tsunami 2005: 26). NRT (2000: 1-1) believes the CIMC structure is designed to work 'equally well' for large or small situations and can 'expand or contract to meet the needs of the incident'.

Regardless of the size of the CIMC, it is important that it focuses on both internal and external communication (ibid: 1-7). In order for the CIMC to operate efficiently and without interruption, it should be organised in such a way as to reduce excesses of ambient noise and to have restricted access. Bernstein (1987: 127) discusses the importance of establishing secure fixed perimeters for the CIMC. He states that ideal locations are schools, city auditoriums, hotels, public arenas or large outdoor tents (ibid.).

\section{Media centre}

A critical element of the media management process is the establishment of a wellequipped media centre run by a dedicated media coordinating group of the lead MECIT. This media centre should be established remotely from the 'flash-point', EOC and CIMC in order to reduce media interference of the overall operations function. According to UK Resilience (2005: 65), this media centre needs to be easily accessible; capable of holding a large number of journalists and their vehicles; be well equipped with appropriate information technology systems, faxes, and multiple 
ISDN/Broadband and telephone lines; and be properly staffed for as long as required, perhaps on a 24-hour, 7-day basis. A suitable space for press conferences should also be available.

The lead MECIT should ensure that media liaison officers (MLO) from each of the principal response agencies are on hand in the media centre to provide journalists with operational updates for respective response organisations. Reynolds (2002: 132) recommends providing background information to the media through 'facts-sheets'. Bernstein (1987: 150) emphasises the importance of preparing 'boiler-plate' press releases and 'holding statements' during the pre-crisis stage that can be quickly adapted and released following the actualisation of a major emergency. Such tools and procedures assist in rapidly filling any information vacuum that may exist. The use of regular press briefings and conferences is a useful method of updating the media. The media coordinating group should also arrange media visits to the 'flashpoint', providing transport and media liaison officers as necessary. AKNZ and EU (2001: 45) suggest the establishment of 'pooling' or sharing arrangements, where necessary, thereby negating the need for media to access sensitive or dangerous sites.

The lead MECIT should appoint a chief spokesperson whose role is 'to manage the accuracy and consistency of the messages' coming from the lead response organisation (Coombs 1999:71). This person should be well trained, fully briefed, accredited, and 'on-message'. According to Mitchell (1986: 17), '... a poorly prepared, trained or unskilled spokesperson can merely exacerbate a major emergency situation'. The subject of media management is discussed further throughout the next two chapters.

\subsubsection{Community outreach and victim liaison}

Community outreach and victim liaison are further key communication functions that require coordination and integration by the MECIT within the CIMC. In this regard, both functions offer a 'boundary-spanning' mechanism between the lead response organisation and the affected community (Sturges 1994: 298). 'Boundary spanning' allows community outreach officers and victim liaison officers to keep a constant 
flow of dialogue with affected publics in order to assess their needs and requirements, and also to gather useful information.

The role of the community outreach officer is to determine the information and other needs of the community; to organise outreach programmes; to make contact with local influential persons who can provide a sense as to how the community perceives the major emergency response. According to Fernandez and Merzer (2003: 155), proactive contact should be made directly with the community itself. This role is often undertaken by personnel other than by communication practitioners and, as such, an excellent information-gathering mechanism may be lost.

Liaison with victims of major emergencies, and their families, is a critical communication function that should be closely coordinated, or at least monitored, by the MECIT. This ensures that the needs and concerns of communities and victims become part of a larger information and communication cycle, thereby ensuring a more focused delivery of action. Without such a coordinated approach, affected publics will not receive the best possible level of care and attention and this is likely to impact on the reputation of the lead response organisation. In the post-crisis stage, the care of victims and their families becomes a particular focus of the media and may result in negative reportage. This subject receives further attention throughout Chapters 7 and 8.

\subsubsection{Building strong relationships}

An important element of the proposed major emergency communication framework is building strong relationships with key stakeholders. Many theorists, including Grunig, Grunig and Dozier (2002: 100), Fearn-Banks (1996: 39) and Coombs (1999: 39) emphasise the importance of building long-term relationships with stakeholders and publics. Marra (1998: 7) believes that an organisation with a strong pre-crisis relationship with its stakeholders, ' ...may suffer less damage than an organisation with weak or non-existent pre-crisis relationships... while research clearly indicates a crisis magnifies poor or non-existent relationships'. In relation to major emergency situations, the objective of building strong relationships prior to the occurrence of a disaster situation is supported by many contemporary theorists. Salter's (1999: 111) 
model (Figure 2.1) acknowledges the importance of communicating with, rather than to, communities. Additionally, Fischhoff's (1995: 138) model (Figure 3.7.4) emphasises the importance of 'making partners' by building up strong relationships with stakeholders. All three major emergency models described earlier in Section 4.5, involve building strong relationships during the pre-crisis stage. Reynolds (2002: 7) supports the 'fostering of alliances', while UK Resilience (2005: 28) and Nordlund (1994: 13) propose 'building relationships'. Building strong relationships takes considerable time and effort, but the majority of major emergency literature provides clear evidence of supporting that task. Susskind and Field (1996:41) note how the cost of building strong relationships with stakeholders, '...may not be obvious in the short term... but if you care about your credibility focus on building strong long-term relationships'.

Lindenmann (1998/99: 19) emphasises that building relationships with stakeholders needs to be managed strategically. Coombs (1999: 45) describes a number of strategic approaches that can be pursued in order to build strong relationships with stakeholders, including staying close through regular contact and two-way dialogue, developing organisational trust and credibility, and consistently meeting stakeholder expectations.

A further important strategic approach is the use of stakeholder maps and networks in order to allow the key stakeholders to be readily identified and contacted. A comprehensive stakeholder network identifies and maps key stakeholders, together with relevant information explaining why these groups are stakeholders, their relationship with the lead response organisation, who to contact in the group and how, and details of who in the MECIT is responsible for contacting them (Coombs 1999: 53; Darrell 2003: 19). Reynolds (2003: 38) emphasises that relationships must be built with all categories of stakeholders, irrespective of their position vis-a-vis the lead response organisation, and includes 'advocates, adversaries and ambivalent'.

Conkey (2004: 44) believes that a major strength of the stakeholder network 'is that a small number of people can effectively coordinate the communication efforts of a large group'. He describes how the Australian government has established an effective stakeholder network in order to communicate critical information to key 
people, who will in turn disseminate this information to others, in the event of a potential 'foot-and-mouth' outbreak in that country. The stakeholder map and network gives the MECIT the ability to contact opinion leaders or spokespersons within particular stakeholder and publics groups quickly and efficiently when seeking to engage in two-way dialogue or to impart important information to them. Coombs (1999: 54) emphasises that, ' ...once established, the stakeholder map and network must be maintained... as neglect of the network leads to eventual problems'.

According to Breakingnews (2004), following the 2004 tsunami in South East Asia, key personnel within the Indian government did not learn of the disaster until it was broadcast on television news. This was due to a failure to maintain and update its stakeholder network and contact details. A senior government aide reportedly stated, 'it looks as if they forgot to update their records' (ibid.).

Of equal importance, according to Darrell (2003: 58) and Norman, Stuart-Black and Coles (2006: 111), is the importance of building strong relationships with the media. Fernandez and Merzer (2003: 30) recognise that building good relationships with the media takes time but in so doing 'can pay dividends in ensuring accurate reporting of the response efforts.' Stronger relations with the media can be built by having regular meetings and briefings, providing consistent speedy responses to their queries, and involving the media in major emergency training and exercises.

Strong relationships, with both stakeholders and the media, are best built during the pre-crisis stage of a major emergency. Marra (1998: 7) states, 'organisations can't build or repair relationships once a major emergency occurs'. Once built, it is extremely important to maintain and further develop these relationships. This can only be achieved through regular contact, particularly personal contact. Coombs (1999: 146) emphasises the importance of including the rebuilding of damaged or strained relationships as part of the post-crisis major emergency communication strategy.

As mentioned in Section 5.3.1, building relationships with stakeholders and publics needs to be considered in the cultural context of the environment within which the communication strategy is being developed and implemented. The purpose and the 
strength of the relationships may vary from country to country. Additionally, the benefits may differ from one country to the next.

\subsubsection{Developing and maintaining trust and credibility}

A key principle that requires careful consideration when developing the major emergency communication framework involves striving for the development and maintenance of trust and credibility amongst key stakeholders and the public. According to Trumbo and McComas (2003: 343), 'credibility and trust are precious commodities' for communication practitioners throughout major emergency situations. Darrell (2003: 20) believes that the most important precondition for successfully handling a major emergency is that the lead response organisation has considerable public trust and credibility. Otherwise, decisions, recommendations and messages produced by the lead response organisation are likely to be met with some scepticism. Nordlund (1994: 13) goes further and states that communication from an untrustworthy response organisation 'may give rise to anger and resentment, or arouse bitter indignation' amongst the public.

O'Brien (2006:14) emphasises that 'the trust of the public is easy to lose, and harder to regain...recovering it is fraught with difficulties'. PR Coalition (2003: 9) insists that rebuilding trust and credibility is 'a long-term undertaking'. Baker and Martinson (2002: 149) believe that high ethical standards are crucial in order to build and maintain trust and credibility. They identify five guiding principles for ensuring an ethical approach: truthfulness, authenticity, respect, equity and social responsibility. Deviation from any of these principles can lead to an erosion of public trust and credibility in the lead response organisation.

For example, in an attempt to reduce public concerns following the 9/11 attacks on the 'Twin Towers', the US Environmental Protection Agency (EPA) announced that 'no significant levels of asbestos in the air had been recorded' (Hidden Killer 2006: 32). However, in February 2006, a Manhattan judge 'blasted the EPA for misleading the public into believing the air was safe' (ibid.). The collapsing tower, she stated, released 'hazardous substances, including fibreglass, freon, mercury and asbestos into the air' (ibid.). The EPA had known within twenty-four hours that asbestos levels 
were four times higher than the acceptable danger threshold. While short-term exposure to asbestos is not dangerous, lies such as this can seriously undermine public trust and credibility in a hitherto trusted organisation.

In diagrammatic format, $\mathrm{CDC}$ provides a useful summary of the significance of source credibility and trust during the major emergency communication process.

Figure 5.2: The importance of trust and credibility to major emergency communication.

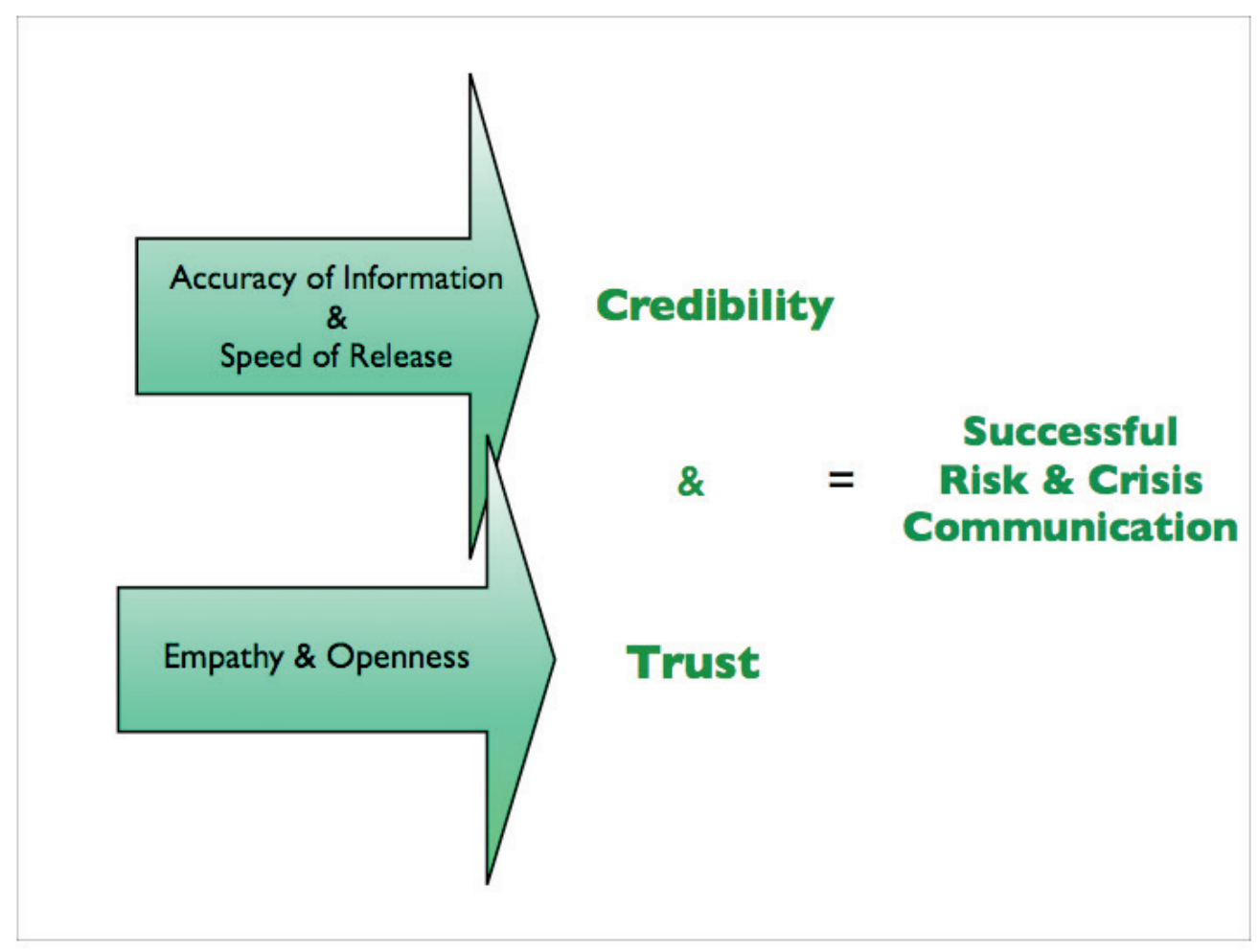

Source: (Reynolds 2002: 39)

From a strategic viewpoint, PR Coalition (2003: 10) describes the importance of measuring levels of trust 'in order to show you where you are in the continuum of change'. Useful tools such as focus groups, surveys, media monitoring, blogmonitoring, and qualitative research can assist in this process.

While it is accepted that building public trust and credibility is an important function that should be carried out during all stages of a major emergency situation, most of 
the effort in that regard is likely in practice to take place during the pre-crisis stage. However, in many countries, government agencies are simply not trusted, particularly amongst certain sectors of society. For example, Lasker (2004: 13) notes that African-Americans place greater trust in community and family groups than in the authorities. Building up trust and credibility is a very difficult task and can only be achieved over the longer-term by the demonstration of ongoing ethical probity, openness, accuracy of information and the matching of positive words with deeds. Additionally, trust and credibility can be quickly and easily lost (as FEMA discovered during their response to Hurricane Katrina). Consequently, response agencies need to have a communication strategy in place for situations where no trust and credibility exists, or where this is lost. This need is discussed in greater detail in the next chapter, which addresses communication during the pre-crisis stage of a major emergency. 


\section{COMMUNICATION DURING THE PRE-CRISIS STAGE}

\subsection{Introduction}

The pre-crisis stage of a major emergency life cycle is the period when governments and lead response organisations must take action in order to prevent and prepare for, potentially life threatening situations. As mentioned earlier, the purpose of this dissertation is to examine whether a comprehensive and sophisticated major emergency risk and crisis communication strategy can assist response organisations in dealing with these situations. In chapter 5, a number of overarching strategic principles that should guide the major emergency communication function were proposed. With these strategic principles in mind, this chapter proposes a series of strategic-tactical steps that the major emergency communication and information team (MECIT) should consider when preparing and planning all aspects of communication during the pre-crisis stage.

In this regard, the MECIT needs to consider communication from two distinct viewpoints: planning comprehensive communication programmes that can assist the lead response organisation in preventing major emergencies from occurring, and preparing all aspects of the communication function in order to support the response effort should a major emergency occur. The management of these two distinct but complementary strands of communication should be considered at two distinct levels, a higher-level and tactical-level.

\subsection{Major emergency communication in the pre-crisis stage}

The development of a comprehensive communication strategy and framework for the pre-crisis stage of a major emergency situation needs to be considered and analysed at two distinct levels, the higher and the tactical. The synergy that exists between the series of strategic-tactical (yellow) pre-crisis major emergency communication steps 
and the overarching strategic communication principles (blue) is evident in Figure 6.1 below.

Figure 6.1: Major emergency communication management - pre-crisis stage.

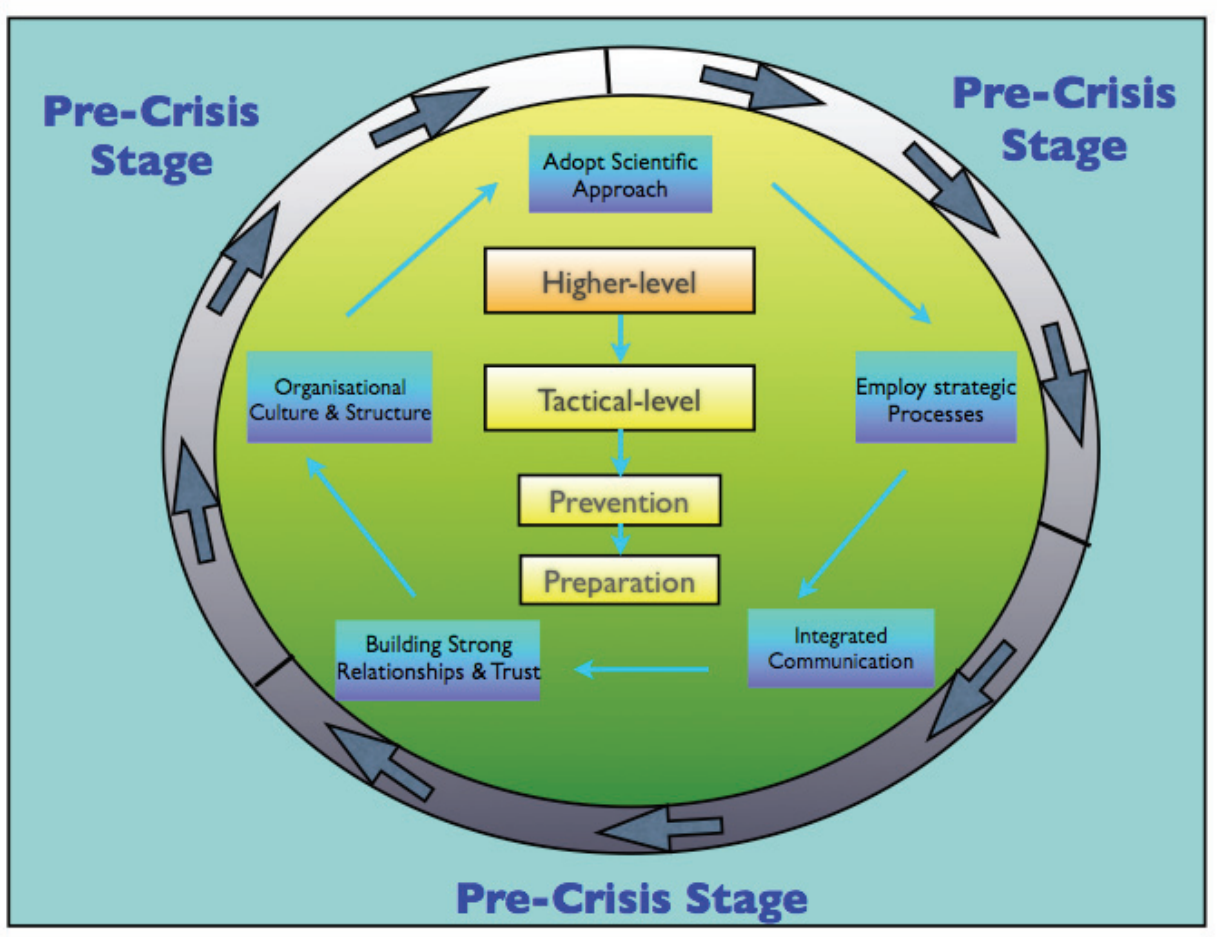

\subsection{Higher-level pre-crisis major emergency communication management}

The pre-crisis stage higher-level involves inceptive but essential strategic-tactical steps that need to be taken by senior decision-makers of the lead response organisation in order to initiate the preliminary stages of the major emergency communication strategy. The higher-level strategy involves three distinct steps.

\subsubsection{Selection of senior communication manager/leader of the MECIT}

Careful selection of the senior communication manager is critical to the success of developing, implementing and leading the entire major emergency communication strategy. In Section 5.2 the importance of empowering and involving the senior 
communication manager at the highest level in the overall decision-making process was discussed. Coombs (1999: 65) offers a useful table that identifies some of the key knowledge, skills and traits that are desirable for the leader of the major emergency communication and information team (MECIT), including having a co-operative disposition, a tolerance for ambiguous situations, and the ability to handle stress. Berge (1990: 44) includes additional desirable traits such as the capability to make swift decisions and to communicate effectively with others. According to Schoenberg (2005: 3), the senior communication manager requires integrity, strategic thinking, persuasive skills, and decisiveness. Additionally, the MECIT leader must possess requisite formal strategic communication knowledge and, ideally, first-hand experience of the role.

Consequently, the choice of the person to lead the MECIT will be determined by the culture and structure of the lead response organisation. However, the choice of a suitable person can greatly influence thereafter how communication is perceived amongst senior decision-makers in the organisation. One of the critical roles of the senior communication manager is that of 'boundary-spanning', acting as a link between the external environment and the senior decision-makers in the lead response organisation.

\subsubsection{Selection of the MECIT}

The next step of the pre-crisis higher-level strategic communication process involves identifying and selecting suitable members for the MECIT. Coombs (1999: 63) describes a team such as this as 'a cross-functional group of people' that has been designated to handle the communication function throughout an emergency situation. In the pre-crisis stage of a major emergency, it is prudent that each individual response organisation develops its own MECIT. It is important that these teams, particularly the lead MECIT, possess a strong mix of creative thought, specialist knowledge, and experience. During the pre-crisis stage, it is likely that the MECIT will consist of a more restricted core group of participants that is augmented and expands as a major emergency becomes imminent or actualises. 
Many theorists, including Fernandez and Merzer (2003: 57) and Bernstein (1987: 21), have described the different skill-sets that should be represented within the MECIT, including leadership, communication, media handling, information management, legal, human resources, community liaison, information and communications technology, finance, risk management, operations and logistics. The MECIT should also have access to expertise in areas such as safety, environmental science, critical incident stress debriefing (CISD) and security. A number of key support staff are also required in the MECIT, including researchers, photographers, switch operators, media monitors, log keepers, website managers, communication and information technology technicians, and copywriters. At a strategic level, the leader of the MECIT should ensure that the overall operational commander understands the value that this diverse pool of skills can provide to the entire response effort and provides the necessary resources accordingly.

\subsubsection{Preliminary goal setting.}

Once established, an important higher-level task for the MECIT, at the very outset, is to conduct an exploratory analysis of the communication environment and to set preliminary goals and objectives. All preliminary communication goals should be closely aligned with the preliminary operational goals of the lead response organisation.

A key preliminary communication goal for the MECIT is the adoption of an appropriate approach and model to guide all aspects of communication with internal and external stakeholders. This goal should stipulate how transparency and openness is to be achieved and how communication should flow throughout the lead response organisation. The MECIT also needs to set a clear goal stipulating that the decisionmaking process involves the use of both 'expert' and 'non-expert' participants. All preliminary goals and objectives are likely to evolve and be refined as the major emergency communication strategy develops. This is likely to occur following a process of continuous communication analysis, deliberation, feedback, evaluation and revision, which are key elements of approaching the discipline strategically. 


\subsection{Pre-crisis stage tactical level}

Once the higher-level pre-crisis communication process is complete, the MECIT can begin to develop and implement each of the tactical-level communication steps-, which involves two fundamental functions. Firstly, developing a communication process that can assist the lead response organisation in preventing a major emergency from happening, or at least to minimise its impact. Secondly, to develop communication processes in order to prepare stakeholders and publics to best deal with the effects of a major emergency situation, and also to prepare the actual communication processes and plans themselves.

\subsubsection{Prevention during the pre-crisis stage}

Major emergency communication management is working at an optimum when it assists the lead response organisation in its risk management and mitigation efforts, thereby preventing major emergency situations from occurring. Coombs (1999: 39) states that the goal of major emergency prevention is 'to defuse the crisis by attending to warning signs and risks'. The Cabinet Office (2004: 28) in the UK emphasises how the communication function can assist in the major emergency prevention role by, '...providing the right people with the right information at the right time in a form that they can understand, assimilate and act upon'.

The MECIT can assist the lead response organisation in preventing major emergencies from occurring by undertaking a number of distinct tasks, including the early warning detection role, the development of stakeholder networks, the building of strong relationships and the fostering of alliances. The final two elements were dealt with in some detail in Chapter 5.

\subsubsection{The early warning detection role}

Through the efficient use of communication, the MECIT can establish a process whereby it can provide the lead response organisation with early warning of hazards that could potentially develop into major emergency situations. Fink (1986: 21) describes how crises can be avoided by recognising 'prodromes' or early warning 
signals. Dozier, Grunig and Grunig (1995: 199) and Darrell (2003: 18) discuss how the process of environmental scanning, 'a systematic and ongoing analysis of the outside world', can provide important intelligence in relation to hazards, thereby giving the lead response organisation adequate time to optimise the decision-making process. However, according to Iwan (1999: 32), this early warning information must be provided in a format that is understandable and of use to the end decision-makers.

Adopting a scientific approach to communication, effective early warning systems can be developed using tools such as stakeholder maps and networks, analysis of the media, focus groups, attitude surveys and opinion polls (Cohn 2000: 114). Grunig, Grunig and Dozier (2002: 550) suggest cyber-scanning, which provides a systematic means of researching potential risks on the internet, as a useful early warning method.

Despite the early identification of serious hazards by the MECIT, many early warning signals tend to be ignored by major emergency managers. Following the Hillsborough soccer stadium disaster in Liverpool in 1989, in which 95 people died, Lord Justice Taylor in his summation to the inquiry highlighted that 'many of the deficiencies in the stadium had already been envisaged' (Taylor 1990: 32). Other examples of warnings being ignored include a devastating famine caused by locust attacks in Niger in 2004, where 'villagers were forced to scavenge anthills hoping to find stray grains of food'. This is despite the receipt of early warning signals of locust attacks on cereal crops in 2003 and 'alarm bells going unheeded' (Walter 2005: 61). Additionally, Thompson (2003: 142) highlights how the US government failed to pick up early warning signals from postal officials prior to the 'anthrax attacks' in 2001. Consequently, the MECIT needs to ensure that any warning signals provided through the environmental scanning programme are acted on by the lead response organisation.

\subsubsection{The building of strong stakeholder relationships and the fostering of alliances.}

As discussed earlier in Chapter 5, building strong stakeholder/public relationships and fostering alliances can help the lead response organisation to prevent certain categories of major emergencies from actualising, or at least to reduce their impact 
when they do occur. For example, in terrorist related incidents, members of the public are more likely to be aware and to pass on warning signals if relationships are strong. Similarly, members of the public are more likely to heed instructing information given by response organisations if time and effort is spent building relationships. This is an important communication function and involves the MECIT identifying useful stakeholders and publics, developing comprehensive stakeholder networks and maps, building credibility and gaining stakeholders and publics trust, engaging in meaningful two-way symmetrical dialogue with them, and ensuring an efficient information management system is in place in order to gather, analyse and action information emanating from the dialogue.

The building of a network of relationships among internal teams at this time is also very important. This helps to ensure that the lead response organisation's 'internal systems of processes and people remain in place and don't collapse under stress' (Schoenberg 2005: 4).

\subsubsection{Communication preparation during the pre-crisis stage of a major emergency}

Despite all of the best efforts of the MECIT and the lead response organisation at prevention, major emergencies will inevitably happen. Coombs (1999: 59) warns major emergency managers that, '...they should not fall victim to hubris [pride] and assume that their preventative measures will protect them from harm'. Consequently, the MECIT needs to assist the lead response organisation to prepare both themselves and stakeholders in order to deal with the effects of a major emergency situation. Effective preparation for a major emergency involves considerable attention to the series of steps contained within the second element of the pre-crisis tactical-level communication strategy. These steps are described below.

\subsubsection{Conduct a detailed major emergency communication analysis}

The MECIT can provide significant assistance to the lead response organisation in carrying out an overall operational analysis in the pre-crisis stage. In this regard, the MECIT effectively utilises many communication tools and functions such as the 
stakeholder network, 'boundary spanning', and information-gathering and analysis. On completion of the operational analysis, the lead response organisation should be capable of identifying potential major emergencies that are considered most likely, and prioritise each of them across such dimensions as 'likelihood of occurrence' and 'extent of impact'. Thereafter, in order of priority, the lead response organisation should set clearly defined operational goals and objectives in order to deal with each identified potential major emergency, and put in place measures to protect its stakeholders and publics.

Taking advantage of the information provided through the operational major emergency analysis, and aligning itself with the goals and objectives set by the lead response organisation, the MECIT should conduct its own detailed major emergency communication analysis. The purpose of this analysis is to assess all available information and to assess the strengths, weaknesses and gaps within the communication management function vis-á-vis each identified and prioritised major emergency. The communication information gathered can be analysed using a number of tools such as the 'assessment worksheet' prepared by Berge (1990: 36). Van Houts (1996: 20) provides an adaptation of the classical 'strengths, weakness, opportunity and threats' (SWOT) model. Using the adapted 'SWOT' model, the MECIT can determine the strengths, weaknesses, opportunities and threats that exist in terms of communication in both the internal and external environment. Adhering to both scientific and strategic principles, the execution of a communication analysis such as this provides the MECIT with the requisite building blocks to develop individual communication strategies and plans for dealing with identified major emergencies.

\subsubsection{Prepare comprehensive communication goals and objectives}

Once the detailed major emergency communication analysis is complete, the next stage in the preparation process is to establish comprehensive communication goals and objectives. These are in addition to, and more detailed than, the setting of the preliminary higher-level goals and objective described earlier in Section 6.3.3. These tactical-level communication goals and objectives should be closely aligned to the 
operational goals and objectives of the lead response organisation, following its detailed analysis of the major emergency environment.

One of the most important goals to be established relates to the choice of communication approach with stakeholders, whether this is reactive or proactive, open or closed. Goals and objectives also need to be clearly set in relation to building public trust and credibility, segmenting strategic publics, developing strategic communication programmes in order to overcome any apparent knowledge gaps identified during the communication analysis, preparing the major emergency communication response plan (MERCP), preparing template communication forms and generic background information, procuring necessary equipment for the CIMC, and training and exercising the MECIT. A final important goal that needs to be clearly stated relates to the involvement of publics in important decision-making concerning major emergency communication management.

\subsubsection{Establishing communication approach parameters}

As discussed earlier in Chapter 2, major emergencies are considered low probability, high consequence events (Flynn 2003: 85), that often occur with a 'big bang' effect (Norman, Stuart-Black and Coles 2006: 5). Consequently, how the MECIT decides to communicate with its publics throughout all stages of a major emergency must be given significant consideration. Many factors can determine the particular approach adopted, including the culture and structure of the lead response organisation, its commitment to approaching the communication function from a strategic and scientific perspective, the stage within the major emergency life cycle, the category of event that society is facing, the duration of lead-in time to impact, and the attitude adopted by the media.

Nohrstedt (2000: 146) highlights the difficulties faced by the MECIT when deciding on the choice of an appropriate approach to major emergency communication. In particular he identifies the 'need for information paradox' and the 'reassure-but-warn paradox' as two major dilemmas that communication managers face when communicating about major emergency situations. This relates, in the first instance, to a sense of apathy displayed by many members of the public when a major 
emergency is not imminent. However, on the other hand, once a major emergency is looming there exists an insatiable desire for information. On both ends of a spectrum, major emergency managers can decide to provide information that may cause unnecessary alarm to the public or can choose instead to understate the danger in an attempt to reassure the public. Bernstein (1987: 82) notes that communicating about risk in major emergency situations 'is never emotionally neutral...it either reassures people or it alarms them'. As shown in Section 2.4.1, a number of diverse factors affect how people perceive risk and danger.

Many contemporary writers, including Covello and Allen (1988: 4), propose that major emergency communication in the pre-crisis stage should involve open, frank, honest and transparent dialogue with publics. Sandman (2004(a)) concurs and stresses that the MECIT should not attempt to over-reassure publics in relation to the potential hazards of major emergencies and instead should 'err on the alarming side' and should aim to avoid 'zero fear'. The Centers for Disease Control (CDC) in the US is also in agreement and states that the MECIT should 'stop trying to allay panic' (Reynolds 2002: 24). For example, it was reported that officials in Indonesia 'covered-up' avian flu and tried to 'deal with it quietly' for two years in order to allay public fear and panic. However, this delay 'merely allowed it to spread among poultry flocks and to people' (Sipress 2005: A01). Sandman (2004(b)) believes that the MECIT should tell people 'what to really expect' in relation to major emergencies. He promotes the principle of engaging in,

....anticipatory guidance...telling people what to expect does raise some anxiety, especially if you're predicting bad news. But being forewarned helps us to cope, it keeps us from feeling blindsided or misled, and it reduces the dispiriting impact of sudden negative events.

Tierney, cited in Alesch et al., (2004: 159) agrees with Sandman and proposes any approach that helps to '...overcome public apathy'.

However, while Fernandez and Merzer (2003: 115) highlight the importance of transparency and openness, they place equal emphasis on an approach that involves 'calming the public... and not unduly alarming them'. This involves a balanced approach and according to the theorists should ensure optimum results for 
communication managers. However, the timing within the major emergency life cycle will be a key deciding factor. If a major emergency is not imminent, such as a 'rising tide' category of major emergency, the MECIT can afford to adopt a more 'persuasive' approach. On the other hand, for a sudden 'big bang' category of major emergency, such as an imminent cyclone, the MECIT may be obliged to use a more alarmist approach in order to save lives. While engaging in 'scare tactics' is beneficial in gaining the public's attention, constantly over-emphasising the risks and dangers of hazards may diminish the credibility of the lead response organisation and its message. Many commentators have accused the Bush administration of 'crying wolf' in relation to terrorism in the US since 2001 in order to secure adequate funding for the president's policies. In the context of establishing communication approach parameters strategically, the MECIT can use scientific communication tools and techniques, such as surveys, focus groups and dialogue, in order to assist them in this important task.

\subsubsection{Redetermine relevant stakeholders and publics}

Once the MECIT has completed its detailed major emergency communication analysis, described earlier (p129), there may be a requirement to re-evaluate, and perhaps revise, the stakeholder map and network (as discussed in Chapter 5). Such an ongoing and iterative process emphasises the strategic approach to major emergency communication management. The MECIT should develop an individual stakeholder map and network for each of the identified potential major emergency situations. These maps and networks should contain details of those people involved in, interested in, or affected by a potential major emergency, and those with specific knowledge or information that may be of use to the decision-making process. Details should also exist of points-of-contact amongst the different stakeholder groups and communities and how best to contact these people. At this stage, stakeholders can be used to identify other potential stakeholders, thereby building an augmented network and map using a multiplier effect. Additionally, for this process to be undertaken at a nationwide level, the lead MECIT should co-ordinate the actions of all regional and local MECITs in order to develop a comprehensive and integrated stakeholder map and network. Useful electronic tools, such as mind mapping, can greatly assist in this process. 


\subsubsection{Message and information formulation}

Once communication goals and objectives have been established for each of the prioritised potential major emergency situations, and the parameters for an appropriate communication approach agreed, the MECIT should then begin the process of preparing, testing, evaluating and revising key messages and information to be eventually disseminated to stakeholders. This process allows the MECIT to inform, advise, instruct, reassure and meaningfully involve all relevant stakeholders and publics in relation to identified major emergency situations.

During the pre-crisis stage of a major emergency, two distinct categories of key messages should be considered. Firstly, 'risk communication messages' can be developed and communicated in order to assist publics to overcome apathy, lack of understanding of major emergency issues, and lead response organisation distrust. Risk communication messages can also inform publics about specific major emergencies that may occur and how they should prepare for these potentially lifethreatening events. Secondly, a number of 'boiler-plate' [generic] crisis communication messages can be developed and tested for each potential major emergency scenario. These 'boiler-plate' messages are then held within the MERCP for dissemination should that particular category of major emergency occur. The messages can be 'position key messages' that convey the government's or lead response organisation's position on what has just occurred, or 'instructional key messages' that are designed to direct stakeholders and publics to take particular action (Fernandez and Merzer 2003: 115). An example of an 'instructional key message' is 'go in, stay in, tune in' that is likely to be issued in the event of a radioactive leak (OEP 2008: 16). The subject of message development will be discussed in greater detail in the next chapter.

\subsubsection{Educating stakeholder groups}

A critical preparedness function during this stage is the requirement for the MECIT to educate key stakeholders and publics about major emergency situations and to raise levels of awareness about personal and collective roles and responsibilities. In the 
event of a major emergency occurring, such preparedness measures encourage publics to behave in a desirable way, thereby promoting the concept of resilient communities. The importance of educating stakeholders in this regard has been emphasised by theorists such as Nielson and Lidstone (1998: 16), Reynolds (2002: 8), Nordlund (1994: 20) and Tarrant (1997: 20). Nussbaum (2005:40) describes the importance of central funding for public education campaigns to prepare for major emergencies, such as extreme terrorist attacks. This would involve 'having a real conversation with the public and that hasn't been done yet.'

The education of stakeholder groups involves important incremental steps by the MECIT that should culminate in getting individuals and communities involved in voluntary preparedness action. In this regard, typical matters that the MECIT should consider during the pre-crisis stage include: educating householders and publics in relation to the types of major emergencies that are most likely and the characteristics of each and immediate protection measures that need be taken; informing them of the actions that have been taken by the lead response organisation to protect the community against different categories of likely major emergency; assessing the types of early warning systems that have been in place and actions to be taken on receipt of a warning or alarm; understanding the various evacuation and shelter procedures in place; the best way to request emergency assistance, information or relief; the means whereby householders can prepare for and protect against major emergencies and survive without assistance for a short period following a major emergency; the best way to deal with psychosocial difficulties such as shock, anger, and post-traumatic stress; and the means whereby householders can assist the lead response organisation and their local communities, particularly in relation to providing voluntary manpower and helping vulnerable individuals. While the benefits of publics possessing this type of information during a major emergency may be clear, there are many difficulties faced by practitioners in disseminating this type of data. In the first instance, the public is exposed to many educational messages, through a range of channels, on subjects including road safety, fire and personal health. These messages are likely to be cognitively prioritised by the individual, based on the relevance and proximity of a message to a particular person at a particular time. Consequently, educational content for major emergency preparedness needs to be relevant to an individual's relationship with a particular emergency 
situation. It can be argued that an apathetic public is unlikely to embrace generic major emergency education. However, targeted educational programmes for citizens, such as those run by FEMA to prepare communities for hurricanes (ICMA 1991:186), may provide a positive correlation between the individual's needs and what is required to survive the first 72 hours of a major emergency. This includes teaching families to have in place survival kits containing water, dried food and a batteryoperated radio.

\section{Education and local resilience}

As members of the public are likely to be the first to arrive at the scene of a major emergency, prior to the arrival of first official responders, it is important that they do not place themselves in unnecessary danger and are available to be deployed to best advantage if deemed necessary by the response agencies. However, this possibility has to be considered within the context of extreme and unprecedented situations, scarce resources, and the need to provide community responders of adequate training, personal protective equipment (PPE), and insurance. At the most fundamental level, it would be ideal if members of communities who decide to respond to major emergencies have some knowledge of what is required and have a basic structure in order to optimise manpower quickly, thereby providing a 'force multiplier' effect. As discussed earlier in Section 2.4.1.2.2, many countries are working closer than previously with their communities in order to train them to protect themselves. For example, in Australia, EMA organises emergency training for young people in remote indigenous communities as part of a government youth strategy (Hocke and O'Brien 2003: 62). In Honduras, members of the community are trained to read simple flood meters in order to measure river levels and provide early warning for severe flooding (Walter 2005: 46). Partnerships could be developed with a number of voluntary and community groups and training may be provided through organisations such as the Civil Defence and the Red Cross. In Ireland, partnerships could be forged with local football clubs that might readily provide an able-bodied response structure to the community. On the basis of the different teams within the club, command and control issues could be easily overcome and groups could be readily deployed on different response tasks. 
Through such partnerships, information may be passed to other community members regarding how best to develop resilience within the community, how to respond in a structured way, and how to assist less able-bodied members. Feedback mechanisms for major emergency planners could also be provided in order to improve response systems and to provide local 'non-expert' information.

\section{Major emergency educational tools}

Public education for major emergency preparedness can be achieved through many diverse channels such as information booklets and DVDs, information stands in public places, advertising, media editorial, and greater engagement with the education system. Following detailed research, prolific organisations such as the UNDRO (1987: 22) emphasise the benefits of disaster education in educational institutions. Shaw et al. (2004: 39) highlight the importance of major emergency education in schools in order to develop ' ... a culture of disaster preparedness, which in turn will urge them to take right decisions and actions as an adult'. Fitzgerald (2000: 1) describes how a culture of major emergency prevention can be built ' ...through education channels so that the youth of today can play an active role in reducing the impact of disasters in the future'. By the careful choice of programmes such as FEMA for kids (FEMA 2008(b)), creating awareness amongst children can be both entertaining and educational and need not involve unnecessary alarm or fear amongst this target group.

Theorists, such as Nielson and Lidstone (1998: 16), discuss the importance of lifelong learning for the individual and for society as a whole. They note when children are educated, parents through their participation in homework and project assignments on the subject of major emergencies can themselves become educated. Additionally, by educating children in this important subject at an early stage, society as a whole is educating its adults of the future. Despite the many demands placed on schools by different interest groups, such as road safety and environmental protection groups, an adequate education of publics in relation to major emergency preparedness has particular merit. 


\subsubsection{Major emergency response communication plan (MERCP).}

By their very nature, many categories of major emergency situations, such as tsunami and earthquakes, are very difficult to predict. Consequently, the MECIT requires a robust series of protocols and procedures in order to initiate effective communication processes, thereby helping to save lives. Accordingly, an extremely important precrisis tactical-level preparation stage function involves the development, by the MECIT, of a comprehensive major emergency response communication plan (MERCP). This plan should systematically address all of the roles, lines of responsibility and resources in order to manage all elements of communication throughout all stages of a major emergency situation. According to Reynolds (2002: 69), the major emergency communication plan, '...more than anything is a resource of information - the go-to place for must-have information'.

Theorists such as Coombs (1999: 60), Pearson and Mitroff (1993: 53), Fearn-Banks (1996: 18), and Fink (1986: 42) describe how many major emergencies possess mutual characteristics. Consequently, a generic plan can be developed, subsequently modified and then adapted to suit a particular major emergency situation, thereby offering a good starting point in order to deal with unforeseen situations. According to Hodgkinson and Stewart (1991: 70):

Clearly, it may not be possible to plan for every disaster scenario. However, the real meaning of preparedness is not about being ready for every possible scenario but the development of core preparedness for eventual crisis situations.

The MERCP should be 'meticulously crafted before a major emergency happens' (ibid: 87). The plan should be a 'manageable and easy-to-read document' (FearnBanks 1996: 24). Significantly, the MERCP needs to be fully integrated into, and 'cross-referenced' with, the lead response organisation's overall operational plan, and additionally with the communication plans of other response agencies (Millar and Smith 2002: 37). According to Reynolds (2002: 69), 'a good communication plan will reflect that co-ordination'. Once developed, the generic MERCP must be continuously updated and revised. In this regard, Coombs (1999: 83) emphasises that 'it must remain a living document'. Additionally, each member of the MECIT must have immediate access to condensed versions of the MERCP for easy reference 
(Millar and Smith 2002: 48). Appendix B contains a proposed major emergency response communication plan (MERCP) structure.

\subsubsection{Major emergency communication exercises and training}

According to Drabek and Hoetmer (1991: 184) ' ...having a plan does not in itself enable an effective response to a major emergency'. In order to ensure that all members of the MECIT, supporting communication teams and associated responders, fully understand all elements of the MERCP, a comprehensive and ongoing programme of integrated communication training and exercising should take place during the pre-crisis stage. In the UK, the Civil Contingencies Secretariat (CCS) (2007: 32) describes how 'training and exercising must underpin planning and embrace the activities of all staff'. According to Fearn-Banks (1996: 25) and Pauchant and Mitroff (1992: 48), crisis team training should involve both individuallevel and group-level knowledge and skills.

Major emergency communication training involves 'equipping people with relevant knowledge and communication skills' to undertake the roles expected of them (CCS 2007: 32). This includes familiarising all staff, particularly new members, with communication protocols, procedures, equipment and systems (Norman, Stuart-Black and Coles 2006: 53). Coombs (1999: 70) believes that training should be specific and that 'people should only be trained in those areas in which they are deficient'. This specific training is particularly important for the spokesperson in order to ensure that he/she appears credible and acceptable to audiences. Fernandez and Merzer (2003: 74) emphasise the importance of training the entire MECIT 'especially in media relations, including practice interviews and press conferences'. The integration of other communication functions in realistic 'scenario-based' training and exercises is also essential, including information managers, internal communicators, community outreach officers, and victim liaison personnel. It is extremely important also to involve the lead response organisation's operational commander in such integrated training with key communication personnel and different response agencies. This allows any lack of synergy between the operational and communication function to be identifed, and also allows the commander to gain an understanding of how best to utilise the MECIT. The provision of appropriate formal courses of training, either in- 
house or external, is also extremely important in order to continuously augment the knowledge-base of the MECIT. Suitable, realistic, and practical training materials must be provided by the lead response organisation for this purpose (EPS 1998: 14).

Major emergency communication exercising, on the other hand, is concerned with 'testing plans and procedures' in order to see if they will work in an actual major emergency (CCS 2007: 32). Millar and Smith (2002: 48) state that by exercising communication plans, the MECIT 'can test to see if the plans actually work', to determine 'if they fit together' and to see if those expected to make the plan work 'understand its content'. Payne (1999: 112) describes how major emergency communication exercises, '... allow contingency plans to be validated...ensuring that all responders are familiar with the contents of both generic and specific plans, together with their own respective roles in those plans'.

Major emergency exercises should be incremental and progressive. At the most basic level, personnel can engage in 'paper-feed' and 'table-top' major emergency communication exercises. These can then be augmented by 'simulation' exercises, in which important aspects of major emergencies can be simulated in order to represent reality and where personnel can respond accordingly. However, full-scale 'live' or 'incident-based' exercises provide the best method of training and exercising (HSE UK 1999: 35). For example Fernandez and Merzer (2003: 75) describe the benefits of 'role-play exercises with the media'. Exercises can also be 'on-site' or 'off-site' and can be designed around an individual agency or can involve a more complex multiagency approach (Payne 1999: 114). Additionally, communication exercises can be 'announced' or 'unannounced', thereby building in an element of surprise for the MECIT and other responders (Drabek and Hoetmer 1991: 189). All major emergency communication exercises must be carefully planned and an exercise outline produced. Additionally, a thorough evaluation should take place and an objective report be produced at the end of each exercise in order to improve communication procedures and processes.

Adhering to the series of strategic pre-crisis steps, outlined in this chapter, allows for a more effective communication response during the crisis stage of a major 
emergency situation. The major emergency response by the MECIT will be discussed in the next chapter. 


\section{COMMUNICATION DURING THE CRISIS STAGE}

\subsection{Introduction}

The crisis stage of a major-emergency life cycle is the period that occurs once an identified significant hazard actualises, becomes a reality, or when affected publics perceive this to be the case. This is the stage that all lead response organisations and supporting response agencies hope to avoid. However, if the authorities are well organised and prepared, a series of strategic steps will have been undertaken during a pre-crisis stage to prevent a major emergency from occurring, or at least to reduce its impact. Unfortunately, these steps are often insufficient and the major emergency enters the crisis stage, resulting in unpredictability, surprise, confusion, fear, great stress, lack of control, an insatiable desire for information, a lack of information, and huge media interest (Marra 1998: 6; Coombs 1999: 3; EPS 1998:7; Nohrstedt 2000: $138)$.

Central to the success of any major emergency response effort is the crucial role of strategic communication management. In this regard, Chapter 7 describes a series of strategic-tactical steps that the major emergency communication and information team (MECIT) undertakes when managing all aspects of an effective and compassionate communication response during the crisis stage. These strategictactical communication steps involve many crucial functions, including helping the lead response organisation to identify and recognise that a major emergency has occurred; the provision of an immediate and effective communication response by the MECIT, which involves the triggering and implementation of the major emergency communication response plan (MERCP); effective communication by the MECIT with stakeholders and affected publics, which includes disseminating warnings and alarms in order to save lives. 


\subsection{Major emergency strategic communication in the crisis stage}

Following the approach taken for the pre-crisis stage, a strategic framework is proposed to guide the actions of the MECIT throughout this extremely demanding phase, the crisis stage. This framework demonstrates how the six overarching strategic communication management principles (in blue \& green), described earlier in Chapter 5, underpin and guide the actions of the MECIT during each of the four strategic-tactical steps (in orange) that are necessary in order to effectively manage communication during this stage.

Figure 7.1: Major emergency communication management - crisis stage

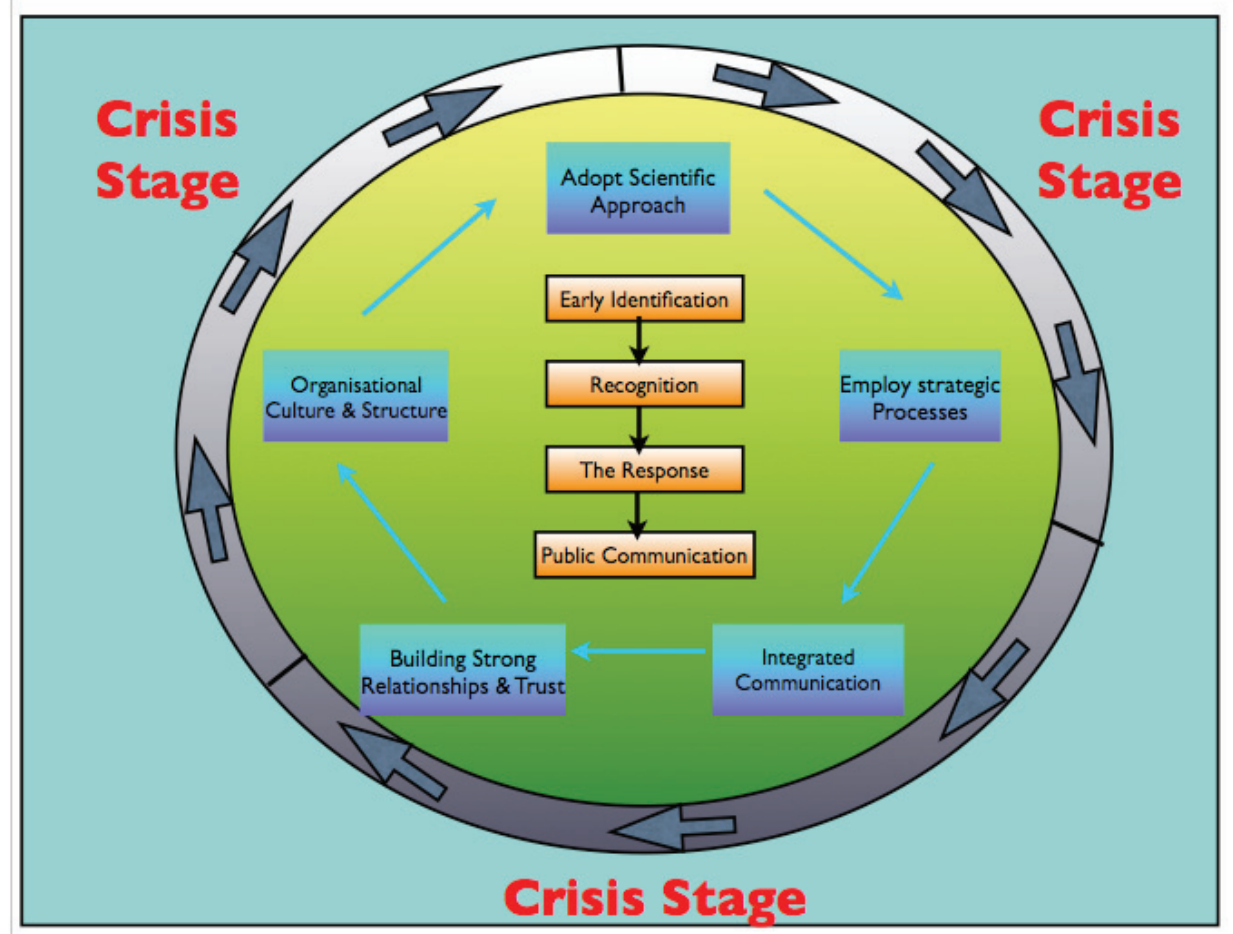

\subsubsection{Early identification of imminent major emergencies}

The first strategic-tactical step taken by the MECIT during the crisis stage of a major emergency involves putting in place communication processes that can assist in confirming that a significant negative event is imminent and is likely to cause considerable death and destruction, and to get this information rapidly to senior 
decision-makers in the lead response organisation. This phase of the crisis stage of a major emergency is closely related to, and is the final task in, the early warning detection role described earlier in Section 6.4.1.1. By adhering to the overarching strategic communication principles described in Chapter 5, such as engaging in ongoing communication research, coordinating across all functional departments and agencies, integrating all key elements of communication such as community liaison and information management, and engaging with 'experts' and 'non-experts' in an information-gathering capacity, the MECIT can put in place an effective process that can both acquire data in relation to an imminent major emergency, and rapidly impart this information to the appropriate decision-maker.

Central to the early identification of imminent major emergencies is an efficient information-gathering and analysing capacity within the MECIT, as already described in Chapter 5. By coordinating with technologically advanced affiliate organisations, the MECIT can ensure that data from the most sophisticated information-gathering tools such as satellite imaging, global positioning systems, remote sensing and meteorological satellite systems, is gathered and processed in order to identify the onset of a major emergency situation. When Hurricane Charley struck Cuba in 2004, more than 70,000 houses were destroyed. Despite the strength of that hurricane, and the subsequent damage caused, only four people died. A significant contributory factor to the preservation of life, in this instance, was Cuba's world-class meteorological service which works in close cooperation with the US National Hurricane Centre, providing major emergency communication teams with necessary information for the early identification and tracking of storms (Walter 2005: 41).

The MECIT can also employ more basic tools such as the use of stakeholder networks in order to identify imminent major emergencies. Good communication flows with diverse sectors of society can prove very beneficial in providing early signals that a major emergency is about to occur. The primitive tribes of the Andaman and Nicobar Islands recognised the onset of the 2004 tsunami from the behaviour of the birds and animals, and the appearance of the sea. They survived by fleeing from the costal areas to higher ground (Walter 2005: 15). In order to save lives, the MECIT needs to establish a process by which it can tap into this type of information, thereby 
providing senior decision-makers in the lead response organisation with information of imminent dangers to the public.

Once a major emergency occurs the MECIT should ensure that they continue with the early identification process in order to detect subsequent secondary crises. As the MECIT may be totally preoccupied in dealing with the primary major emergency, it may not have sufficient resources to undertake a detailed early identification process for secondary crises. A rapid identification of these secondary major emergencies may help to alleviate greater levels of suffering for already affected communities. The United Nations quickly identified a secondary major emergency situation following the earthquake in Kashmir on October $8^{\text {th }} 2005$. A senior UN official warned that $90 \%$ of the tents given to earthquake survivors were not equipped for the harsh Himalayan winter. The UN also recognised that the most serious risk was posed to those survivors still remaining in mountain villages rather than in the refugee camps. Early identification of this secondary crisis is reputed to have saved many lives and reduced much suffering (Owen 2005: 4).

\subsection{Major emergency recognition}

The process of major emergency recognition is closely related to the identification function discussed in the last section. Major emergency recognition involves being able to report to an appropriate senior decision-maker within the lead response organisation that a major emergency is imminent, or has just occurred, and that this fact is recognised, accepted and positive action taken. This process may involve the MECIT having to prove to the senior decision-maker that a major emergency situation exists. An efficient integrated communication and information management system established by the MECIT can greatly assist the lead response organisation in recognising that a major emergency has just occurred.

Unfortunately, the recognition process is not as straightforward as it seems. In some instances a major emergency may have been identified and reported, but for different reasons it has not been received by the appropriate person or office and therefore cannot be recognised and acted on. For example, in Thailand, the government-run 
Earthquake Bureau failed to open an e-mail sent from the tsunami alert centre in Hawaii at $9.04 \mathrm{am}$, identifying the impending 2004 tsunami. This would have given the authorities one hour's notice thereby allowing them time to send a warning alarm. Unfortunately, major emergency recognition was arrived at only after many lives were lost (Drummond 2005: 6). Some major emergencies are easier to recognise than others. For example, 'big-bang' major emergencies, as described by Norman, StuartBlack and Coles (2006: 5), are very obvious and recognisable due to the fact that the devastation happens almost immediately. In cases such as these, the identification and recognition process occur simultaneously. The Kobe earthquake in 1995 that caused the deaths of 6,000 people, injured more than 50,000, and destroyed approximately 100,000 homes, was quickly identified and simultaneously recognised by authorities as a major emergency.

However, Coombs (1999: 89) notes that all major emergencies are 'not so obvious' and may not be readily recognised by lead response organisations for a variety of reasons. This failure to recognise that a major emergency has occurred may prevent an adequate crisis response, thereby jeopardising efforts to protect affected publics. Describing a not-so-obvious major emergency, Norman, Stuart-Black and Coles (2006: 5) identify a 'rising-tide' category of major emergency, where the problem 'creeps up gradually and has no clear starting point' (ibid.). This category is difficult to recognise and the point when it becomes a major emergency may only become clear in hindsight. According to Kamer (1996: 64), a lead response organisation 'may not even know that it has reached the crisis stage of a major emergency'. In the case of influenza pandemic, confirmation of the fact that it has arrived in a particular country may be difficult to ascertain and it may take some time before it is deemed a major emergency.

Some lead response organisations may recognise the existence of a major emergency, but may wish to deny that it exists. The Indonesian government recognised the existence of 'bird flu' in its country. However, in order to allay panic amongst its publics, it chose to deny its existence for two years (Sipress 2005: A01). In some instances a major emergency of significant impact may have occurred but due to inexperience, lack of training, or denial the authorities may fail to recognise its impact. 
Additionally, media attention can create widespread recognition of a major emergency and act as a catalyst for appropriate action. According to the International Red Cross, in January 2005 the dire situation in Darfur, Sudan, had still not been recognised as a major emergency by the International Community (Flanagan 2005: 2). Walter (2005: 137) states:

There was no massacre aftermath video on the scale of the Nyarabuye church in Rwanda in 2004 where 20,000 Tutsis and moderate Hutus were slaughtered...however the harassment, the beatings, the robbery, the rape, the murder continue on a daily basis and unfortunately it continues well below the radar screen of the international media and of the international diplomatic machine... by the time we're absolutely sure what's happening in Darfur is genocide, it will be too late.

However, intervention by Hollywood stars such as George Clooney, who 'made an impassioned plea for action in Darfur' to the United Nations Security Council in September 2006, created much badly needed recognition that a major emergency exists in that country (BBC 2006).

As with the early identification process, the MECIT should have an ongoing and systematic communication process in place in order to collect and process data that can assist the lead response organisation to recognise that a major emergency has occurred. According to Coombs (1999: 89), the MECIT can contribute to the major emergency recognition role by,

...collecting data, converting the raw data into usable information, storing the information, and relaying the necessary information to internal and, perhaps, to external organisational stakeholders - especially expert and government organisations.

As major emergency recognition involves an accurate and detailed report reaching an appropriate senior decision-maker to the effect that a disaster situation has occurred, it is essential that effective channels of communication exist for this information to flow quickly. Consequently, a comprehensive and up-to-date internal communication network must exist from the lowest response level to the highest, which must be operational at all times. This must be integrated with the external stakeholder network in order to allow information to be received quickly from key organisations such as international police, weather stations, seismic stations, and also from members of the 
public. This is a critical role for the MECIT during the crisis stage of a major emergency.

\subsection{Major emergency communication response}

Much has been written on the importance of a speedy and effective communication response by the MECIT following the actualisation of a major emergency, including contributions from theorists such as Bernstein (1987: 97), Coombs (1999: 118), Levick and Smith (2005: 5), and Darrell (2003: 40). Fernandez and Merzer (2003: 109) note how the lead response organisation's handling of the first 24 -hours '... will establish its reputation and credibility for managing the entire response and recovery operation'.

Norman, Stuart-Black and Coles (2006: 117) term this 24-hour period the 'goldenhour'. Once the lead response organisation identifies and recognises that a major emergency situation is imminent, or has occurred, a number of immediate strategictactical communication steps need to be taken, most of which occurs pretty much simultaneously. Darrell (2003: 40) states that the first immediate reaction of the senior communication manager should be to trigger the major emergency communication response plan (MERCP) and to alert and activate the members of the MECIT. Reynolds (2002: 90) also emphasises how the lead response organisation should ensure that an effective communication process is in place in order to allow it to inform and activate its own chain-of-command, together with those of partner/affiliate response organisations. A process such as this allows these response organisations to trigger their respective major emergency operational and communication plans. Bernstein (1987: 115) suggests the use of a 'cascading notification system' in order to quickly notify many people in the event of a major emergency. As described earlier in Chapter 5, the MECIT should quickly establish itself in a well-equipped and suitably secluded emergency operation centre (EOC), or if required a communication and information management centre (CIMC). Locations such as these provide a central hub for information collection, analysis and dissemination, and for the co-ordination of the entire communication effort. This provides the foundation for 'a cohesive communication response' (Fernandez and 
Merzer 2003: 112). If spokespersons have not already been chosen, it is necessary to task a principal, together with a number of secondary spokespersons, who are accredited to speak on behalf of the entire response effort. The use of a small number of well-trained spokespersons helps to ensure consistency in any messages disseminated to stakeholders, publics and the media. The spokespersons need to be thoroughly briefed and rehearsed in all aspects of the situation and the response.

Once these important initial strategic-tactical steps have been carried out, the MECIT must then work closely with the lead response organisation and other affiliate communication teams in order to begin the communication and information management process. This includes establishing immediate communication goals and objectives that are closely aligned to those of the overall response effort. This is likely to involve a revision and re-establishment of any pre-crisis communication goals and objectives described earlier in Chapter 6. As information is gathered and analysed, and the nature of the major emergency situation becomes clearer, the MECIT also needs to revise and tailor its generic MERCP in order to deal with the specific situation at hand.

At this stage, it is also important to ensure that technical communications systems within the lead response organisation, and between other affiliate response agencies, are operational. While this function is primarily an operational/CIT task, the MECIT should liaise with the relevant sections in order to ensure that adequate technical systems exist to allow the free flow of data throughout the entire response effort. One of the most significant problems encountered while coordinating the responses to both the 9/11 and the London bombings was a breakdown in internal technical communications. An internal inquiry following the London Bombings reported:

Police, fire and ambulance staff all used different radio systems and rescuers at ground level could not talk to their colleagues underground (BBC 2007).

Consequently, many countries are moving towards implementing a unified, interoperable, nationwide, public safety radio system that can be utilised by the principal response agencies, including fire, police and ambulance services. For example, the 'terrestrial trunk radio' (TETRA) system, based on the 'global system 
for mobile communication' (GSM) technology will be introduced to the emergency services of Norway in 2011 (Siemens 2008). UK response agencies are also introducing a system that will allow communication with responders operating in the London underground system (BBC 2007).

Employing many of the overarching strategic communication principles, such as detailed communication research and evaluation, managing communication by objectives, and making important decisions by way of a public engagement process, may be difficult to undertake due to the intense time pressures and resource demands generated by the actualisation of the major emergency situation. A balance needs to be achieved between ensuring that communication is approached strategically and that action is taken promptly and decisively. The senior communication manager is likely to require supplementary communication personnel and equipment in order to enable the communication management process to be approached strategically rather than undertaking a mere tactical approach. Likewise, the MECIT needs to consider applying the strategic communication principles in a more condensed manner.

\subsection{Communication with stakeholders and affected communities}

The fourth and final strategic-tactical step that the MECIT must consider during the crisis stage of a major emergency is how best to communicate with stakeholders and affected communities. Good communication during the crisis stage of a major emergency involves '...providing the right people with the right information at the right time in a form they can understand, assimilate and act upon' (HM Government 2005(b): 31). Reynolds (2003: 6) describes five communication failures that can 'kill operational success' during the crisis stage:

Mixed messages from multiple experts, information released late, paternalistic attitudes, not countering rumours and myths in real time, and public power struggles and confusion.

A critical success factor in the communication process during this stage, according to Reynolds (2002: 89), is that the lead response organisation makes every effort to become the acknowledged source of information, particularl 
$y$ for the media and affected publics. This involves minimising any delay in making an official statement following the occurrence of a major emergency. Being the recognised source of information also requires communicating with a broad range of people, each requiring different types of information, with different degrees of urgency displaying openness and transparency where possible. The most urgent group in this regard is the community that is likely to be affected by the major emergency. According to the CCS (2007), this group 'needs to know' critical information such as basic details of the incident, the implications for their health and safety, clear advice and guidance on how to protect themselves, and reassurance that help is at hand. This group also 'wants to know', details of practical implications such as any effects on transport and utilities, a helpline number, and what is being done to rectify the situation (ibid.).

Other important stakeholders that require communication during the crisis stage include cabinet ministers, national and local elected representatives, senior civil servants, local authorities, emergency services, fellow response workers and other members of the internal audience, the families of response workers, healthcare professionals, community leaders, trade and industry leaders, managers of essential public utilities and essential services, the international community, and media organisations. Reynolds (2003: 39) emphasises that the MECIT is also obliged to communicate with publics who are not supportive of the response effort or the lead response organisation.

\subsubsection{The crisis stage communication process}

Crisis communication with stakeholders and affected communities should begin as soon as a major emergency has been identified and recognised. 'It is important to avoid the bunker effect' (CRN 2003: 35). Any delay in communicating 'will be perceived as stalling' (Bernstein 1987: 42). The MECIT should ensure the immediate release of pre- prepared material or preliminary verified information, in order to be perceived as the first and principal source of 'credible' information and also to 'fill the likely information vacuum' (Fernandez and Merzer 2003: 110; Murray and Greenwood 1993: 52). A speedy communication response can demonstrate the lead response organisation's capability in taking action and responding to the major 
emergency (Coombs 1999: 116). According to Reynolds, (2003: 5) the MECIT leader must quickly decide '... what to release, when to release it, how to release it, where to release it, who to release it to, and why release it'. A strategic approach to decision making, as described in Chapter 5, and realistic 'scenario-based' exercises can greatly assist in this process.

\subsubsection{Communication channels}

The MECIT should 'start quickly and open information channels' once a major emergency occurs (Darrell 2003: 58). It is generally accepted that the most efficient means of communicating to publics is through the print and electronic media. Consequently, great attention should be given to optimising media output during the crisis stage. The development of good working relationships with the media in the pre-crisis stage can greatly assist in this regard. The important subject of media management was discussed earlier in Section 5.3.5.4.

While the media is an excellent channel for reaching stakeholders and affected communities during the crisis stage of a major emergency, other potential channels of communication should be carefully considered by the MECIT. In this regard, useful channels of communication include advertising, mail-shots, websites, telephone, mobile phone, pager, short messaging services (SMS), hoardings, digital signage, loudspeaker, and leaflet drops. Channels such as these allow stakeholders and affected publics to receive key messages and critical information through as many different conduits as possible, thereby reinforcing and confirming the significance of the information to the intended recipient. Additionally, 'interpersonal communication' (as discussed in Chapter 3) with affected publics is an extremely important means of disseminating information following a major emergency, '...particularly for certain segments of the population such as children, the infirm, the elderly, those who may fail to understand a particular message, or those without access to mass media sources' (Rogers 1989: 25).

Forms of interpersonal communication channels that are considered effective during the crisis stage of a major emergency include public meetings, outreach programmes, door-to-door contact, self-help groups, public information bureaus, and engaging with 
publics through free-phone numbers. However, an optimum method of message penetration involves the employment by the MECIT of a mix of interpersonal, mass media, technology and other communication channels.

A speedy communication response to stakeholders and affected communities is ever more important as 'technology has accelerated the spread of information thereby reducing the amount of time for responding' (Coombs 1999: 114). Brown (2003: 23), commenting on his experiences of dealing with Hurricane Isabel (2003) in the United States, agrees that a speedy communication response is essential. However he does recommend that the MECIT should perhaps ' ...step back and take time to think for a moment...especially about key messages'.

\subsubsection{Development of key messages and information}

\subsubsection{Key message development}

As mentioned in Section 5.3.5.2, a significant problem faced by the MECIT is the development of key messages and useful information from the profusion of incoming unfiltered raw data to the communication and information management centre (CIMC). Central to the success of the entire communication process, during the crisis stage, is an effective system for filtering and processing information for message development. Chess, Calia, and O’Neill (2004: 106) recommend a process of 'communication triage' whereby the MECIT clinically prioritises all information to be analysed, prepared and disseminated. In this regard, it must be decided, '... which populations are the most important to reach?...when?...and what information is the most critical to convey' (ibid.).

As described earlier in Section 6.4.2.5, much of the work involved in developing key messages should have been completed during the pre-crisis stage of a major emergency, when the MECIT has more time available for this important task. This allows the MECIT time to adopt a strategic approach to the task, utilising techniques such as 'brain-storming' and 'story-boards' to assist in the message development process. Additionally, in order to ensure the widest base of knowledge is employed, the MECIT should engage in meaningful public dialogue, thereby making use of both 'expert' and 'non-expert' opinion. This ensures that key messages, when 
communicated, are effective and more readily acceptable to the intended recipients. Many theorists, including Price (1989), Stern and Fineberg (1996), Nohrstedt (2000), Lasker (2004), Tierney, cited in Alesch et al., (2004), Reynolds (2002) and Susskind and Field (1999) have written in detail on this subject.

\subsubsection{Communication research}

According to Cushman and Sanderson King (2001: 86), people only understand major emergencies in terms of their own experiences, ' .... and once you go outside people's experiences, not only do you not communicate you cause confusion' (ibid.). As seen in Section 3.4.3, different people are likely to react to distressing information in different ways for a number of different reasons. To gain a better understanding of how diverse groups are likely to react or behave, it is necessary for the MECIT to approach the communication management process strategically and to carefully segment the general public into distinct stakeholders and publics. Despite the time pressures and resource constraints imposed on the MECIT, Reynolds (2002: 37) emphasises how public segmentation and demographic considerations are especially relevant during the crisis stage. She identifies a number of critical factors that provide a useful basis for the segmentation process, including geographical location, experience of previous major emergency situations, education, age, gender, and cultural and socio-economic factors. In order to ensure that messages are relevant to each diverse group of stakeholders and publics, the MECIT should engage in thorough research and develop messages in terms that are clear, simple, understandable, relevant and appropriate for each identifiable segment. The MECIT must try to avoid the 'selective perception' phenomenon occurring amongst intended recipients, whereby parts of disseminated information only are assimilated, and any critical data that is perceived to be irrelevant to the recipient is ignored. Likewise, 'cognitive dissonance' must be avoided, whereby the intended recipient ignores any information that does not fit his/her pre-conceived ideas or beliefs. Once the MECIT knows to whom it is communicating, it can then develop a number of categories of messages, including 'position key messages' and 'instructional key messages', already mentioned in Section 6.4.2.5. 


\subsubsection{Message content}

In terms of message content, Reynolds (2002: 38) emphasises the importance of '...getting the facts right, repeating them consistently, and avoiding sketchy details early-on'. Tierney, cited in Alesch et al., (2004: 172) believes:

Complete accuracy in documents and messages is key...even small or seemingly unimportant inaccuracies may call into doubt the reliability of the entire message.

The London Metropolitan Police released inaccurate information concerning the cause of the first blast on the London Underground in July 2005. Claiming that this blast was caused by a 'power-surge' rather than a terrorist bomb, the police may have seriously damaged their credibility for future message dissemination (BBC 2005). According to Nohrstedt (2000: 143), ' ...there is nothing so destructive of confidence than to be caught-up in a lie... being found out in some kind of cover-up is the kissof-death'. Pillittere, cited in Cushman and Sanderson-King, (2001: 93) describes how 'truth is not only important, but it is one of the first things upon which one will be judged'. Consequently, it cannot be over-emphasised the importance of accuracy, openness and honesty, in the development and dissemination of all messages throughout all stages of a major emergency. If inaccuracies have occurred in message content, Sandman and Lanard (2007) emphasise the importance of acknowledging and apologising for these errors, deficiencies and mistakes.

In a situation where information content appears incomplete or inadequate, the MECIT leader must decide whether it is preferable to wait and not to communicate until more information is available. However, contemporary teaching indicates that some form of initial communication to stakeholders and affected communities is important. Additionally, this initial message should contain a caveat stating clearly that the information is preliminary and incomplete. Fernandez and Merzer (2003: 110) emphasise the importance of admitting 'I don't know' when major emergency information is not yet confirmed. 


\subsubsection{Testing key messages}

Despite considerable time pressures and resource constraints, and cognisant of approaching communication strategically, the MECIT should endeavour to test and evaluate all key messages prior to dissemination. The use of focus groups proves the most efficient method of achieving this when time is limited. These messages may need further brainstorming, revision and possibly another round of evaluation following the testing process. Adopting such a process reduces the possibility of the intended recipient rejecting the key message and provide a greater guarantee of them behaving in a desired manner. In this regard, Connelly and Knuth (1998: 650) suggest an ongoing communication process with diverse communities, including those of different races, ethnic backgrounds and incomes, in order to undertake thorough message research, testing and evaluation.

\subsubsection{Key message dissemination}

Once key messages have been developed, the MECIT must ensure that they are wholly consistent, in terms of content and tone during dissemination. According to Reynolds (2002: 38), ' $\ldots$..consistent messages are vital...inconsistent messages will increase anxiety and will quickly torpedo the credibility of experts'. Nordlund (1994: 29) discusses how messages that 'contradict previous statements can affect the credibility of the lead response organisation'. Tierney, cited in Alesch et al., (2004: 172) highlights how all messages disseminated should be consistent across time, as well as across different message sources and channels. Additionally, the body language used by spokespersons needs to be consistent with the content of the message being disseminated. Furthermore, Nordlund (1994: 29) describes how contradictory messages from sources other than accredited spokespersons can undermine the validity of the officially disseminated message. The necessity for regular briefings and updates for the MECIT, all members of the lead response organisation, and all supporting MECITs, is essential in order to avoid mixedmessages. 


\subsubsection{Empathy for affected publics}

In the development and delivery of their key messages, the MECIT should ensure that the lead response organisation does not appear uncaring or remote to the plight of affected communities. It is extremely important that the language, content and tone of delivered messages do not minimise the severity of the situation for the victims, that sufficient concern and dedication to resolve the major emergency is displayed, and that reassurance is provided to the public that the lead response organisation has sufficient expertise and competence to handle the situation professionally (Reynolds 2003: 11). Darrell (2003: 22) describes this as demonstrating social sensitivity, where there is a show of fundamental respect and empathy displayed to affected publics.

Following the nuclear accident in Chernobyl in 1986, the Soviet authorities were accused of insensitivity in their communication with affected publics. Their administration denied that a disaster had occurred in the first instance, and then accepted no responsibility for the situation thereafter (Mould 1988: 11). Similarly, Levick and Smith (2005: 5) describe the length of time it took for President Bush to 'express deep sympathy for the victims' following Hurricane Katrina. This delay, they claim, 'probably increased the administration's political isolation and public resentment' (ibid.).

\subsubsection{The major emergency public warning process}

A crucial strategic-tactical element of the public communication process, during the crisis stage of a major emergency, is the issue of public warnings, warning alarms and instructing information. According to Norhstedt (2000: 140), the warning phase begins '...when catastrophe looms overhead... which may be followed shortly by the sounding of a warning alarm'. While the terms major emergency 'warnings' and 'warning alarms' are somewhat similar and often used interchangeably, Nordlund (1994: 23) differentiates quite clearly between both. A warning, he describes, is 'a prognosis...prior notice of something unpleasant that may be going to occur'. A warning alarm, on the other hand, is also as a warning, however, '...it is one that is generally accompanied by operative measures... and alerts the receiver to present danger or an acute situation that may exist'. 
Clear and detailed protocols in relation to the issue of warnings and warning alarms should be formally recorded in the major emergency response communication plan (MERCP) in order to avoid confusion at this important stage. This should include a decision as to which response organisation is best placed to issue the warning, and most importantly, who has ultimate authority to sanction this order. This important information should be understood by all members of the MECIT and key personnel of the lead response organisation.

\subsubsection{Issuing public warnings, alarms and instructing information}

\section{Public warnings}

Nordlund (1994: 23) describes two different categories of major emergency public warnings that can be issued. 'Specific warnings', he states, refer to obvious threats such as a hurricane about to 'make landfall'. 'General warnings', on the other hand, relate to broader threats such as the probable risk of a terrorist attack. Morge (2000: 3) states that the issuing of public warnings 'at the right time and to the right target group ...can be a matter of life or death'. NSTC (2000: 9) concur and highlight how effective warnings allow people to "take actions that can save lives, minimise damage, reduce human suffering and speed recovery'. Additionally, they emphasise that these warnings ' ... should reach, in a timely fashion, every person at risk who needs to be warned, no matter what they are doing or where they are located' (ibid: 6). Furthermore, Nordlund (1994: 23) notes:

A warning should activate previously stored preparedness information in the mind of the individual citizen... should conform to existing major emergency communication planning...should also describe the threat, indicate when the event will occur, and outline what people should do.

Consequently, the effectiveness of a major emergency warning depends, to an extent, on the level of preparedness of stakeholders and affected communities and of their individual and collective perceptions regarding the hazard and how it is likely to affect them. Section 3.4.3 has already identified many variables that are likely to influence people's risk perception. One of the most difficult barriers to overcome in relation to major emergency warnings is public apathy. An example of this relates to 'specific warnings' issued prior to Hurricane Katrina making landfall, when 
Lieutenant Kevin Cowen, Press and Information Officer for the New Orleans police, stated:

The difficulty is getting the public to believe our warnings of an incoming disaster, especially when these warnings come as the sun is shining. They just don't believe you (Cowen 2005).

The dissemination of a warning alarm or alert can help to focus the attention of any complacent or apathetic publics.

\section{Warning alarms}

The presence of a well-conceived alarm system provides a very efficient method of warning publics of the immediate onset of a potentially disastrous situation.

Examples of warning alarms include the network of horns dispersed throughout the Netherlands to warn of breaches in the Polder dyke system, and in Bangladesh, where the use of whistles provide an efficient alarm system for imminent cyclones (McGuire 2004: 10).

Advances in technology provide an excellent platform for the dissemination of warning alarms to affected publics. One of the most efficient methods of issuing a warning alarm is through television and radio broadcast. Walter (2005: 32) describes how technology, especially the Internet and mobile phones, 'provides exciting opportunities' for the issue of warning alarms and alerts. For example, the use of Short Messaging System (SMS) on mobile phones offers a rapid means of providing a warning alarm to the vast majority of people in developed countries. Landline telephones can also offer an efficient alarm system. The Thurston County telephone alert system, in the US state of Washington, provides a telephone messaging service every fifteen minutes to alert affected publics of impending floods in their specific areas (Thurston County 2006).

However, warning alarm systems do not exist without inherent difficulties. Alarms are useful to an affected community only if they are timely. Significant problems can arise if publics rely on warning alarm systems and these alarms are given too late, or else not at all. For example, in Thailand and India, authorities issued warning alarms too late during the 2004 Tsunami and many deaths and much suffering followed (Drummond 2005: 6). Another difficulty that arises with warning alarms involves a 
level of public complacency as a result of exposure to previous 'false alarms'. According to Smelser and Mitchell (2002: 39), '...false alarms and misdirection of warnings to people not at risk tend to generate public apathy and hostility'.

Following the issue of warning alarms to evacuate New Orleans during Hurricane Katrina, many residents decided to remain in their homes as a result of receiving similar 'false alarms' on previous occasions (Dejevsky 2005: 20). Warning alarms that are confusing or contradictory may also pose a significant problem. The Kemeny Commission that investigated the 1979 crisis at Three Mile Island nuclear power station in Pennsylvania stated:

During the first few minutes of the accident, more than 100 alarms went off, and there was no system for suppressing the unimportant signals so that operators could concentrate on the significant alarms. Information was not presented in a clear and sufficiently understandable form (Augustine 2000: 21).

The dissemination of warning alarms requires considerable attention from the MECIT during the pre-crisis stage of a major emergency. Issues that the MECIT need to consider include, choosing the most efficient alarm system that is capable of reaching all affected publics; putting in place authorisation procedures for triggering a warning alarm; developing guidelines to overcome delays in disseminating the alarm, particularly in relation to the establishment of service level agreements with broadcast media outlets and external communication and information technology (CIT) providers.

Warning alarms are only effective if the public understand their meaning and react accordingly. Consequently, the MECIT needs to make its communication decisions employing a broad-based system of dialogue, using both 'expert' and 'non-expert' knowledge, in order to determine the most effective warning alarm system that should be employed. Thorough research and evaluation should also be conducted in order to determine how particular alarm systems can be improved and how people are likely to behave once an alarm is disseminated. Any system of warning alarm that is employed should be thoroughly tested and evaluated prior to using it for a live situation. This emphasises once again that the strategic communication principles are to the forefront during this important strategic-tactical step of the crisis stage. 


\section{Instructing information}

Once a warning or alarm has been disseminated, it may be necessary for the intended recipients to be given information by the MECIT explaining what the warning or alarm signifies, what consequences the imminent/occurring major emergency is likely to have for them, what likelihood there is that the receiver or their families are directly or indirectly affected, what actions they should take, where can they receive more information, and what is being done to protect them against harm (Nordlund 1994: 25). This is a form of warning information and is often referred to as 'instructing information'. Bernstein (1987: 91) emphasises that all instructing information must be 'clear and unambiguous and cannot be misinterpreted'. Confusing instructions were given to victims in the 'Twin Towers' immediately following the 9/11 attacks. Recordings of telephone conversations revealed that instructions given to victims were contradictory. According to Eggen:

We were told to use damp towels to keep out the smoke, to break -- or not break -- windows and, in numerous instances, to simply lay low and stay put. Information available to the workers was often sketchy or inaccurate; some operators initially thought a helicopter, rather than a jetliner, had crashed into the North Tower (Eggen 2006: A03).

In order to ensure that instructing information is not confusing, and is efficient when disseminated, Lasker (2004: 24) provides a useful insight into the importance of testing the information on people not closely involved in its development.

Instructing information can also involve giving advice and instructions to members of the public in order to protect and provide them with relief from the effects of a major emergency (Sturges 1994: 300). Typical instructions include guidance in relation to evacuation and protection from the effects of a major emergency. For example, in the period immediately following the July 2005 London bombings, police issued instructing information to Londoners to remain at work, or at home, and to avoid all but the most essential journeys (Staunton 2005:1). Similarly, Walter (2005: 85) talks of instructing information given to the survivors of the 2004 tsunami in Sri Lanka, in relation to safe drinking water. 


\subsubsection{Likely behaviour of affected publics}

Following the dissemination of a major emergency warning, alarm or instructing information, Nohrstedt (2000: 140) describes as 'widely varying' the likely behaviour of stakeholders and affected communities when '...some publics are traumatised while others can display a range of emotions from apathy to manic hyperactivity'. Smelser and Mitchell (2002: 40) identify a number of other emotions that can arise, including disbelief, denial, emotional numbing, fear, anger, guilt, and in some cases, mental disorders. Morrisey and Reser (2003: 56) highlight how the warning phase can often be more distressing than the actual impact. Interestingly, despite an often-held perception that panic ensues following the issue of a major emergency warning alarm, Nohrstedt (2000: 140) and Gist and Lubin (1989: 20) note how research demonstrates that panic is relatively uncommon and that people often react quite rationally even under extreme severe stress. Scanlon (1997: 2) agrees and states that 'the real problem is not panic but the absence of panic', when people are more likely to discount warnings than be panicked by them.

A number of other theorists such as Shaw (2001: 5), Canter (1980: 71) and Reynolds (2002: 24) support the proposition that people who are exposed to a major emergency situation not only behave logically, but are likely to be supportive, helpful and altruistic. Fischer (2002: 124) describes how survivors of major emergencies are likely to ' ... share their tools, food, equipment and especially their time...they search for the injured, the dead, and they begin clean-up activities'.

The conduct of communication research into people's likely behaviour following the issue of major emergency warnings, alarms or instructing information needs to be completed, in the most-part, during the pre-crisis stage. However, an objective, onthe-ground assessment of how publics actually react once a major emergency occurs, must also be completed by the MECIT in order to assist the decision-making process. This is especially important if the crisis stage is protracted and if secondary major emergency situations are likely. Once again, this important task is difficult to complete due to time pressures and resource constraints that arise as a result of the major emergency situation. The development of strong stakeholder relationships can assist the MECIT in this difficult task. Additionally, the use of focus groups proves 
useful in order to receive feedback from stakeholders, affected publics, and significantly, the media. Engaging in such a process allows key messages and instructing information to be evaluated and revised if necessary, identifies any gaps in the current knowledge-base to be filled, helps to counter rumours, and helps to assess the concerns, needs and perceptions of the different audiences.

Possessing useful knowledge, such as this, can greatly assist the MECIT to communicate effectively with stakeholders and affected communities during the final stage of the major emergency life cycle, the post-crisis stage. This will be discussed in the next chapter. 


\section{COMMUNICATION DURING THE POST-CRISIS STAGE}

\subsection{Introduction}

Nordlund (1994: 37) describes the post-crisis stage of a major emergency as the period that occurs 'once the acute crisis has passed or subsided and the situation has begun to stabilise'. The post-crisis stage can be sub-divided into the period immediately following a major emergency and the longer-term relief and recovery period. Both of these periods involve the provision of sensitive and timely assistance to stakeholders and affected communities by a variety of diverse agencies both governmental and non-governmental, co-ordinated by the lead response organisation. According to the Home Office (2008:1) in the United Kingdom, the lead response organisation during this period is most likely to be the local authority.

The major emergency communication and information team (MECIT) can greatly support the lead response organisation in its efforts during the post-crisis period. Similar to the process adopted for the pre-crisis and crisis stages, the MECIT follows a series of strategic-tactical steps in order to communicate effectively with stakeholders and affected communities during the post-crisis stage of a major emergency situation. These strategic-tactical communication steps are essential in order to restore a sense of normality as soon as possible and indicate how the MECIT needs to engage in proactive and sustained communication with stakeholders and affected communities, particularly victims and their families. The steps also indicate the benefits of communication in the restoration of essential services and economic activity for the affected communities. Significantly, during the post-crisis stage, an essential task for the MECIT is to improve the entire strategic communication process for future major emergencies. An additional task for the MECIT, described in the fourth strategic-tactical step, is the establishment of a communication-based major emergency tracking and early warning function, similar to the process described in the pre-crisis stage. The final step in the post-crisis stage involves closing the major emergency communication life cycle and placing the entire communication function, once again, on a pre-crisis footing. 
Despite its importance, there has been very little written on the subject of strategic communication management for this important, demanding and potentially protracted post-crisis stage. Furthermore, it proves extremely difficult to identify any comprehensive, integrated strategic major emergency communication framework that can serve as a guide for practitioners during this stage of a major emergency event.

\subsection{Major emergency strategic communication in the post-crisis stage}

In this section, a strategic framework is proposed that provides guidance for practitioners responsible for managing all of the strategic-tactical communication functions during the post-crisis stage of a major emergency, when communication with stakeholders and affected communities needs to be efficient, open and compassionate. As with the previous two chapters, the critical communication functions that need to be undertaken during this period are presented in the form of a series of progressive steps. Figure 8.1 (over), demonstrates clearly how each of the five strategic-tactical steps (in pink) proposed in this framework is underpinned by the six strategic communication principles (in blue \& green), described earlier in Chapter 5. 
Figure 8.1. Major emergency communication management - post-crisis

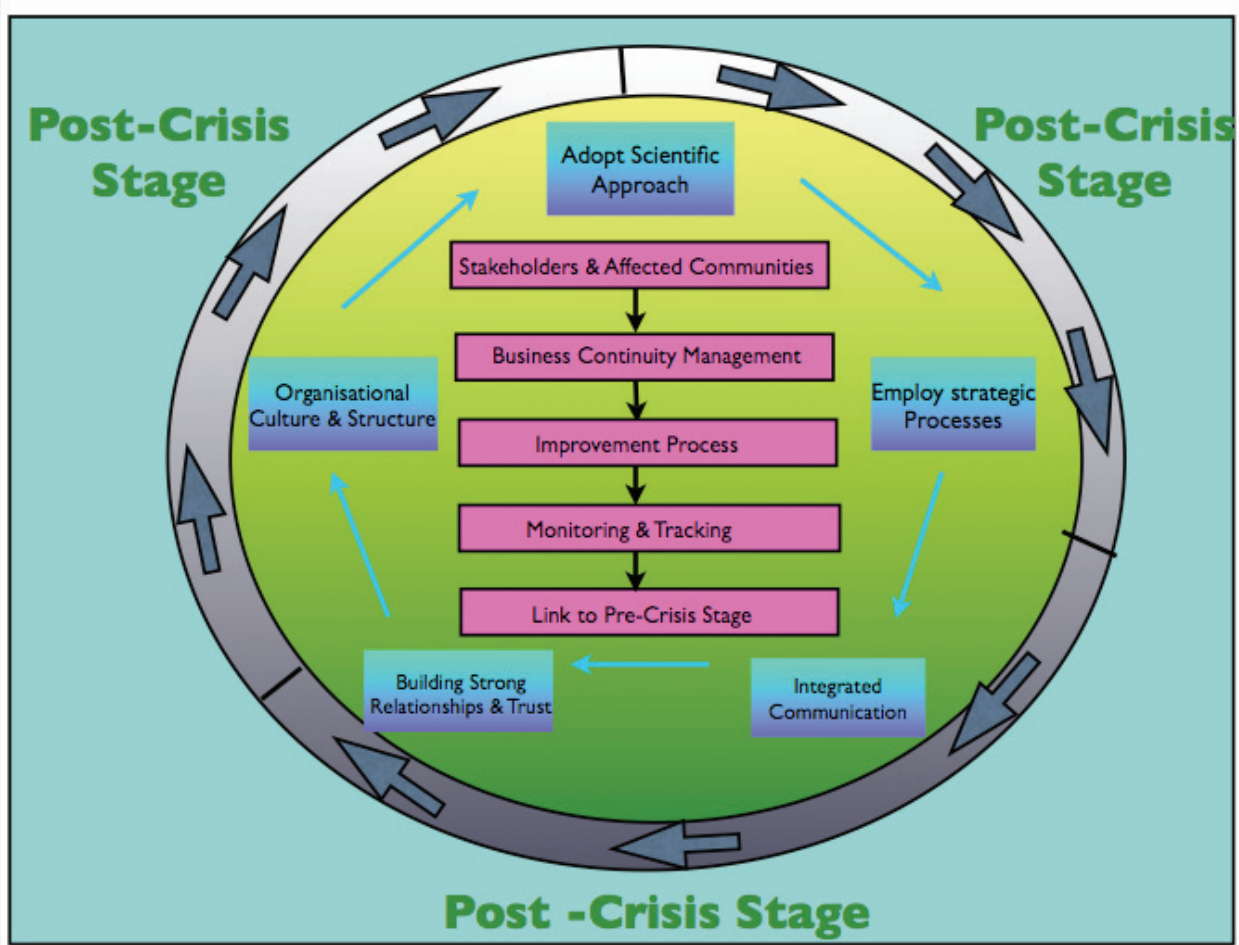

\subsection{Communicating with stakeholders and affected communities}

\subsubsection{Dealing with information demands}

Communication practitioners within the MECIT, operating from the communication and information management centre (CIMC), are likely to have to deal with a range of complex information demands from various stakeholders and affected communities during this stage. Consequently, the MECIT, working closely with the other information and communication teams within the CIMC, should begin the process of dealing with the post-crisis information demands of stakeholders and affected communities and delivering key messages in order to support the recovery and relief operation.

One of the principal information demands at this time concerns public safety. As soon as is feasibly possible, the MECIT needs to communicate to publics that the current major emergency situation has passed and that the direct threat has receded. This type 
of message can only be signalled by the MECIT following close consultation with the operational commander of the lead response organisation using clear protocols that have been laid down and agreed by all during the pre-crisis stage. It is imperative that no support or affiliate organisation issues an 'all clear' message that proves to be premature.

People will look to the lead response organisation at this time in order to ascertain the cause of the major emergency. According to Ogrizek and Guillery:

One of the core demands from stakeholders and publics is for the truth about the causes of the incident and legal acknowledgement or attribution of responsibility (1999: 60).

Other types of information likely to be needed during this period include questions about the likelihood of secondary emergencies, safety and precautionary measures that stakeholders and affected communities should be taking, contact details for aid and assistance, measures that the lead response organisation has taken to prevent any further damaging events, an indication as to when normal public utilities and services are likely to resume, the plans in place for re-building and repairing any damage and an assessment of whether the community is likely to recover fully. Additionally, the public may also have questions relating to the provision of welfare support, alternative accommodation, and litigation matters.

\subsubsection{How to deal with information demands}

The MECIT needs to put in place an efficient process for gathering and accurately determining the information demands of stakeholders and affected communities during the post-crisis stage. The adoption of a comprehensive integrated communication and information management system, described earlier in Chapter 5, can assist in this regard. To accurately ascertain the requirements of different groups, the MECIT can employ useful communication tools such as focus groups, one-to-one meetings with community leaders, small group discussions, 'town hall' meetings, telephone hotlines, electronic bulletin boards, information centres, and surveys. However, to fully comprehend the perceptions, issues, needs and concerns of the victims, their families and communities, it is necessary that face-to-face dialogue takes place between trained community outreach and victim liaison officers from the 
MECIT and affected publics, in order to receive first-hand feedback. An up-to-date stakeholder network and map within the major emergency response communication plan (MERCP), clearly identifying the many diverse audiences together with key contact points amongst these groups, can greatly assist this process. According to the Home Office (2008: 8), engaging in such a process also allows the lead response organisation to engage fully with the community and elected representatives in all aspects of recovery management such as building projects and how best to spend public donations.

Once an information demand is received in the CIMC, the required information can be quickly processed by the MECIT, prioritised against the other information demands, and appropriate decisions reached as regards what should be said, who should say it and through what channel. A broad range of expertise, including legal, medical, financial, and logistical must be available to the MECIT in order to provide guidance and clarity on all of the information demands received. A clearly understood system of protocols should also exist for signing-off messages and information prior to releasing them to stakeholders and affected communities. It is extremely important that any information provided to stakeholders and affected communities that contain either explicit or implicit promises are followed-up and thereafter honoured by the lead response organisation.

Through a process of comprehensive and careful strategic communication planning, using methods such as 'scenario planning' (described in Section 5.3.4.3), the many different types of information demands should have been envisaged, prioritised and recorded by the MECIT during the pre-crisis stage. Effective protocols and procedures should have been developed and included within a comprehensive communication plan in order to allow the MECIT to deal efficiently with these information demands. Additionally, dealing with complex information demands should have been rehearsed during pre-crisis integrated communication training. 


\subsubsection{Victims and families}

According to Ogrizek and Guillery (1999: 60), '...victim management during the post-crisis stage is one of the biggest challenges, if not the central challenge, of major emergency communication'. Effective communication with victims and their families during the post-crisis stage of a major emergency often requires greater levels of sensitivity than in the previous two stages. Consequently, it merits detailed attention at this stage of the dissertation.

\subsubsection{Sensitivity and empathy}

As the post-crisis stage of a major emergency situation is often associated with death, injury and suffering, the development and dissemination of many messages may need careful, sensitive and compassionate consideration by the MECIT. For example, specific messages may have to be crafted in order to deal with traumatised children, religious and cultural taboos, and informing families of the death of loved-ones who may be awaiting the positive identification of remains. Numerous examples have been reported of families of victims having experienced insensitive communication from response agencies that were merely following bureaucratic procedures or badly conceived protocols. Following the terrorist attacks in New York in 2001, the lead response organisation acknowledged that hospitals had insufficient training and skill for dealing with the queries, and communicating adequately with, families who were frantically searching for loved-ones. This caused great distress for the families concerned and placed great pressures on the response organisations (EMA 2003: 11). It was also reported that many of the next-of-kin of victims were 'coldly' asked to fill out forms authorising authorities to 'dispose of any tissue samples' of loved-ones found at the 'Twin Towers' site (Bernstein 2002: 255). In another example, BoucherHayes, reporting for RTE from Thailand following the 2004 tsunami, describes how 'excessive bureaucratic red tape' created significant delays in the repatriation of victims' bodies to their home countries, thereby generating a sense of 'overwhelming anger and anguish' amongst families (Boucher-Hayes 2005). Connors (2003) also describes many examples of gross insensitivity displayed by military authorities while communicating with families of soldiers killed in combat. In order to communicate to victims' families that their loved ones are not forgotten, the lead 
response organisation must consider longer-term issues such as creating a book or website of condolence, a suitable memorial design and anniversary services (Home Office 2008: 5). A co-ordinated response from all of the communication teams working within the CIMC, utilising a broad-range of knowledge, skill and experience, advised by 'expert' and 'non-expert' opinion, can greatly influence and help to avoid unnecessary, additional grief for the families of victims.

The role of the spokesperson is crucial in ensuring that messages disseminated during the post-crisis stage are accurate, informative and sensitive. Likewise, all spokespersons for support response agencies must be fully briefed to display a similar level of empathy. Coordination such as this ensures that the lead response organisation does not appear uncaring or distant from the plight of victims and their families. Unfortunately, despite best intentions, one well-publicised negative story of insensitive treatment of a victim, or the family of a victim, by a relief and recovery official is often sufficient to tarnish the reputation of the entire lead response organisation.

\subsubsection{Marginalised groups}

In the aftermath of a major emergency situation, the MECIT must ensure that it communicates adequately with all members of affected communities. The vast majority of interested and affected audiences will never be more demanding for information than during this very difficult period. However, poorly conceived major emergency planning may result in marginalised groups being excluded from the communication process for a variety of different reasons. Consequently, their needs and concerns may not be assessed, their opinions not sought and important information may not be communicated to them. Often these groups are not identified in the first instance, or they are merely ignored. These marginalised publics are often the sector of society that most require assistance during a major emergency, and include people suffering from physical mobility, sensory deprivation, learning difficulties, and psychiatric problems. Other marginalised groups requiring special attention during a major emergency situation include infants, the travelling community, ethnic minorities, and the aged. 
Drabek and Hoetmer (1991: 179) emphasise that the MECIT must devise a 'viable programme' during the pre-crisis stage in order to obtain and update registration of these marginalised groups. Norman (2004: 92) states that effective recovery can be achieved only through the process of community resilience. Stewart (1989: 27) believes that marginalised people feel less threatened and more empowered by the provision and receipt of information through self-help associations that are organised through community resilience programmes. In this regard, the MECRP should contain information relating to marginalised groups together with details of voluntary community organisations in the region that can offer them local assistance. This information should be updated as quickly as possible by community outreach officers in the post-crisis stage and all efforts should be made to improve communication flows to, and between, these marginalised people.

Walter (2005: 106), describing information flows in South East India following the 2004 tsunami, reports how marginalised groups such as Dalits or low caste 'untouchables' were excluded from the information process. Likewise, the Federal Emergency Management Agency's (FEMA) communication system failed 32 elderly residents in a nursing home 'who were left to fend for themselves and died in the floodwaters' in St. Bernard's Parish, New Orleans, following Hurricane Katrina (Gross 2005: 3).

\subsubsection{Post-crisis communication channels}

The MECIT of the lead response organisation in the CIMC should have considered, as part of the major emergency response communication plan (MERCP), optimal channels for disseminating critical information to stakeholders, victims and their families, marginalised groups, internal audiences and to the general public. In addition to traditional communication channels such as television, radio and the print media, the MECIT needs to consider a whole range of new information and communication technology (ICT) as potential information channels. These include digital media (radio and television) channels, the Internet, 'short messaging services' (SMS) text, 'SMS push-text', 'wireless application protocol' (WAP), 'text-to-listen' and 'smart' pagers. 
However, despite the benefits of both traditional and new technologically based channels, in its planning the MECIT must consider that a major emergency situation can destroy many of these channels. Shaw and Sinha (2003: 41) describe how the 'entire telecommunication system' was destroyed in the Gujarat earthquake in India (2001), thereby excluding many significant communication channels. Furthermore, many marginalised groups may not have access to ICT channels of communication (Fischer 1999: 48). Consequently, the MECIT should plan for a series of effective non-technical channels of communication. For example, the use of boats equipped with megaphone systems proved effective in passing important information to many of the residents of New Orleans following Hurricane Katrina. Similarly, using nontechnical means, the posting of photos of unidentified victims on billboards allowed the responders in Thailand to communicate information to a large number of families and friends following the 2004 tsunami (Scanlon 2006: 58). In a similar way, victims who survived, and wished to inform their relatives that they were alive, also posted notices on public buildings (Berger 2004: 11). Walter (2005: 106), reports how community-based civil society networks, such as fishermen's organisations using door-to-door visits, provided an extremely effective channel of communication in the post-crisis stage of the 2004 tsunami in Sri-Lanka.

\subsubsection{VIP visits}

The presence of community leaders / VIPs 'is an inevitable sight at the site of a major emergency' (Home Office 2008: 5). Visits of this type can provide a significant morale boost for both victims and responders, reinforce key messages, and places on the public record gratitude for the entire relief and recovery effort. In some instances a VIP visit provides a catalyst for badly needed fund-raising towards the relief effort, such as the visit of former US presidents Clinton and Bush (Snr.) to South East Asia following the 2004 tsunami.

Any visits by community leaders / VIPs should be co-ordinated by the MECIT of the lead response organisation. In this regard, they should ensure that any visits do not interfere with the ongoing relief and recovery effort. The MECIT must also be cognisant that community leaders / VIPs may be obliged to answer difficult questions from victims and families in relation to how the major emergency was 'allowed' to 
happen, or receive criticism relating to the relief and recovery effort. It is important that any communication with these stakeholders and affected communities does not appear defensive or indifferent. Community leaders / VIPs are likely to be pressed to make specific commitments to affected communities during these visits. Care must be taken not to create any unrealistic expectations and to follow through with any commitments given. Failure to do so may significantly damage the credibility of the leader and the lead response organisation in the future.

\subsubsection{Communicating with the media}

Many major emergency events, such as earthquakes and tsunami, are likely to occur and terminate within a matter of minutes, resulting in media organisations only being able to provide coverage of the post-crisis period. Consequently, the MECIT of the lead response organisation is often faced with the task of dealing with an extremely large media presence at multiple locations, very quickly, during the post-crisis stage. Effective coordination of all media activities by the lead MECIT within the CIMC is extremely important in order to ensure optimal utilisation of media output. The handling of the media once they begin to gather at the site of a major emergency was discussed in some detail throughout Section 5.3.5.4.

The focus of media interest in the post-crisis stage often centres on finding someone who is culpable for allowing the major emergency to happen and criticising authorities for intransigence. A number of theorists criticise the media for propagating myths during the post-crisis stage such as 'sensationalist' reportage concerning mass panic following the 2004 tsunami (Sandman 2004(b)), dead bodies spreading disease (Walter 2005: 87), and mass looting and rape in New Orleans following Hurricane Katrina (Gold 2005: 10). According to Monbiot (2005: 15), media reportage during this stage often involves ' ...tales of heroic rescue teams battling to extract people before they die. Best of all, someone might survive against all the odds'. For example Chakravarti (2005: 14) reports in The Times the case of the 12-year-old Indian boy who was 'remarkably' found alive on a coconut tree after nearly two weeks after the 2004 tsunami. The Times reported how he had kept alive 'eating the fruit of the palm and drinking coconut water'. 
Due to the competitive nature of the media market and the relatively short attention span of the non-affected public, journalists and reporters are likely to lose interest in the relief and recovery effort once no new angles appear for their reportage. As soon as the next major emergency arises, the media is likely to shift its focus of attention away from the current crisis. Consequently, the general public often fails to gain an insight into the longer-term difficulties experienced by victims and their families, such as the large number of homeless remaining in South East Asia three years after the 2004 tsunami. As a result, the MECIT needs continuously to work with the media in order to ensure that any reportage benefits the longer-term relief and recovery operation.

\subsubsection{Communicating with major emergency responders and their families}

Communicating with major emergency responders and their families during the crisis stage of a major emergency has already received attention in the previous two chapters. Continuation of this crucial communication process is important during the post-crisis stage, particularly if response workers have been killed, injured or are missing. Additionally, as a major emergency that lasts for more than a few days is likely to impact heavily on human resources, effective internal communication processes can assist in ensuring that staff are available to deal with these events over the medium- to long-term. According to Fernandez and Merzer (2003: 153), emergency responders and their families are important ambassadors and messengers for the lead response organisation. With the 'right information', they believe that these important stakeholders ' ... can play a role in shaping perceptions throughout the entire community' (ibid.). Consequently, internal communication is a critical element within this framework, and is an important task that must be co-ordinated by the MECIT throughout the post-crisis stage, regardless of how protracted this period is.

Critical to the success of communication with major emergency responders and their families is an effective process of continuous two-way symmetrical dialogue. This allows an important uninterrupted flow of information between the lead response organisation and its internal audience. The MECIT should provide essential information, such as progress reports, updates of new threats, and proposed plans, directly to response teams and indirectly to their families, prior to releasing this 
information to the media. This imbues the responders and their families with a sense that their efforts are being appreciated by the lead response organisation.

Additionally, a process must exist that allows the responders and their families to ask questions and to receive honest and detailed answers. Additionally, useful information can be received regarding the concerns and needs of individual response workers, particularly in relation to occupational health issues that might require the immediate intervention of medical, psychological or critical incident stress debriefing (CISD) expertise.

Particular care must be afforded by the MECIT to correctly communicating with the families of responders who are seriously injured or killed in the line of duty. This includes efficient protocols for breaking the bad news, the identification of the body, dealing with cultural or religious peculiarities, providing support at any funeral services, providing financial and social support, and dealing with the media (Connors 2003).

\subsection{Communicating business continuity management (BCM)}

HM Government (2005(a): 75) defines business continuity management (BCM) as '... a flexible framework designed to help critical businesses to continue operating in the face of, and following, a major emergency'. Examples include providers of essential utilities such as electricity, water, natural gas, and other critical services such as transport, shelter and food products. BCM is a process that is extremely germane during the post-crisis stage of a major emergency situation. Scarman Centre (2004: 8-31) emphasises:

BCM should not be considered as merely a plan but an ongoing process... and is not an attempt to continue all operations in all circumstances, but aims to ensure continuance of critical processes in order to aid the wider recovery process.

According to HM Government (2005(a): 90), the role of communication in relation to $\mathrm{BCM}$ in the post-crisis stage includes, communicating the rationale and significance of invoking the business continuity plan; a list of what constitutes a critical business; establishing and recording points of contact within each critical businesses; actions to 
be taken in the event of a BCM plan being invoked; and the level of service that can be expected from individual businesses following a major emergency. A two-way flow of information between the lead response organisation and the critical organisations, through the liaison teams, is essential for BCM success. This type of open information flow allows the needs and concerns of staff within the critical business to become known, thereby prompting the provision of essential support and material that can include such diverse requests as personal protective equipment (PPE) and vaccinations.

EMA (2003:11) describes how the implementation of a comprehensive BCM plan was essential to the recovery of the Manhattan region following the 9/11 attacks. The 'Twin Towers' and environs housed much of New York's financial district, law courts, City Hall, and other important retail and social outlets. Through a process of direct liaison, the authorities provided advice on the immediate re-establishment of many of these services in alternative locations, as they were considered critical to the resumption of normal economic and social activity.

The MECIT also needs to consider the provision of advice to the non-critical business community, in relation to appropriate actions that can be taken to support the overall relief and recovery effort in the post-crisis stage. For example, communication experts advised Pepsi Cola to discontinue an advertisement featuring people surfing following the 2004 tsunami disaster, as it could have been perceived as insensitive (Adland 2005). Furthermore, the provision of information to stakeholders and affected communities in relation to the resumption of critical services is essential to restore public confidence in the lead response organisation, the government, and the business organisations themselves.

\subsection{Improvement of the major emergency communication strategy and plan}

Chapter 5 provides a description of how an effective evaluation of the entire communication process can greatly enhance and improve the major emergency communication effort, thereby helping to save lives and reduce damage. This includes 
an evaluation of whether the strategic communication function has contributed to supporting the lead response organisation's efforts throughout all phases of the major emergency. However, in order to improve objectively the major emergency communication process, it is necessary for the MECIT to collate the findings of the evaluation, revise all sub-optimal communication protocols and procedures, and to implement changes.

\subsubsection{Evaluation of all elements of the communication process}

According to Price (1989: 163), a comprehensive evaluation is 'a necessary step in ensuring improvements in the competence of communication programmes'. While the strategic communication process involves engaging in ongoing and iterative evaluation throughout the entire major emergency cycle, it is likely that the most comprehensive communication evaluation will take place during the post-crisis stage. The MECIT can conduct a comprehensive evaluation of the entire major emergency communication strategy from a number of distinct viewpoints. Coombs (1999: 136) emphasises that a 'natural link' exists between all forms of communication evaluation. Firstly, the MECIT can evaluate the efficacy of the overall communication strategy of the lead response organisation. This involves identifying any inherent weaknesses that may have become apparent in the approach taken to major emergency communication, or in the quality of the delivery of the communication strategy at any particular stage. It includes an evaluation of the quality of the major emergency response communication plan (MERCP), how this plan is implemented by the MECIT, at both individual and group level and of any internal or external factors that can inhibit optimal communication performance.

Secondly, the MECIT can evaluate the impact of the major emergency communication effort. According to Sen and Egelhoff (1991: 71), this evaluation should also be conducted in order to ascertain the effectiveness of the strategic communication process. Ogrizek and Guillery (1999: 80) emphasise the importance of evaluating both short- and medium-term impacts of the communication effort. In this regard, evaluating physical impacts of major emergency situations, including human and environmental damage, should be a key indicator of how successful the communication effort actually is. A question in this regard is whether a more 
effective communication strategy and plan, or MECIT performance, could have prevented or reduced loss of life or material damage. Another type of impact evaluation that is considered important during this stage involves determining how the reputation of the lead response organisation may have been affected. According to Coombs (1999: 140), reputation evaluation requires the measurement of pre- and post-crisis major emergency reputation scores.

Closely linked to the evaluation of reputation is a detailed assessment of media coverage and output concerning the handling of the major emergency situation. According to Susskind and Field (1996: 211), portrayal by the media of the lead response organisation's handling of the major emergency, along with the duration of this type of coverage, can be extremely important in creating positive or negative perceptions of the relief and recovery effort amongst stakeholders and affected communities. Media coverage, when it is negative and of a lengthy duration, is likely to impact adversely on the reputation of the response effort and the lead response organisation. A thorough evaluation of international, national, regional, local, and ethnic media output can assist in identifying any damage that may have occurred to the reputation of the lead response organisation. This type of evaluation can also be a catalyst for taking positive steps to raise and rebuild the trust and credibility of the lead response organisation amongst stakeholders, the affected community and the media themselves. Undertaking a comprehensive media analysis during the postcrisis stage involves techniques such as media content and share-of-voice analysis, qualitative in-depth discussions with senior media representatives, and focus groups. Undertaking an evaluation such as this can assist the overall evaluation effort and offer an excellent forum for learning important lessons and improving media relations for future major emergency communication management.

In order to evaluate effectively the efficacy of the communication strategy, the evaluation team from the MECIT should refer back to the strategic communication goals and objectives that were stated during the pre-crisis stage. Bernstein (1987: 19) emphasises the importance of agreeing and stating 'clear criteria that should be used as a benchmark' during the evaluation phase in order to determine whether a particular element of the strategic communication process can be considered a success or failure. Norman (2004: 95) concurs and specifies a number of criteria that 
can be used to evaluate the success of the strategic communication process. Reynolds (2002: 87) suggests the use of tools such as a strengths, weaknesses, opportunities, and threats models (SWOT) in order to conduct a comprehensive evaluation of the major emergency communication strategy process. Helsloot and Ruitenberg (2004: 107) propose an appraisal of citizen feedback as a means of determining the success or failure of major emergency communication.

Similarly, Coombs (1999: 136) states that 'data collection is the first step in any evaluation process'. A principal source for evaluation data is the many records that should have been maintained throughout the entire major emergency period. Useful information can be gleaned by the MECIT through a comprehensive audit of records such as stakeholder contact worksheets, incident report sheets, information log sheets and rumour logs. Additionally, another useful method includes an evaluation of direct stakeholder feedback from both internal stakeholders - such as civil servants, members of the emergency services, and affiliate support agencies, and external stakeholders - including victims and the media. Information from external stakeholders can be acquired through the use of structured surveys, interviews, focus groups, internet sites, 'blogs', media output and media log sheets.

The gathering of this type of data provides essential information concerning the effectiveness of many aspects of the strategic communication process and any shortcomings that may exist. These include, the effectiveness of notification processes, information flows to responders and between response organisations, delays in gathering or assessing information, the effectiveness of individual channels of communication, gaps in the supply of information, places where particular messages are not being understood by receivers, how stakeholder queries have been dealt with, and the extent of participative collaboration in decision-making. Theorists who have written on this subject include Fernandez and Merzer (2003: 174), Bernstein (1987: 139), Coombs (1999: 136), Reynolds (2002: 54), and Ogrizek and Guillery (1999: 80).

Once information has been gathered, Mitroff, Harrington and Gai (1996: 45) suggest a number of variables that can be used for organising the data in order to assist the MECIT with the evaluation process. This involves organising the information into 
specific categories, including the type of major emergency, the stage within the lifecycle, different systems and technology utilised, and various groupings of stakeholders and publics. Engaging in a strategic approach to evaluation, as discussed in Chapter 5, can greatly assist the MECIT to decide where deficits have occurred and how best to revise the existing major emergency communication strategy and the MERCP.

Notwithstanding the importance of the evaluation process, the conducting and completion of an effective communication evaluation following a major emergency situation may prove difficult. Resources are likely to be scarce and communications staff fatigued. Consequently, from a practical perspective, each element that requires evaluation should be prioritised and an objective cost-benefit analysis conducted in order to determine whether it is worth proceeding.

\subsubsection{Revise communication strategy and plan, and implement changes}

The results of the comprehensive evaluation of the communication process should be collated and reported to senior decision-makers within the lead response organisation and other relevant and interested response organisations as soon as possible. These results should then form the basis for a detailed revision of the major emergency communication strategy, and plan (MERCP), and be used to strengthen inherent weaknesses within the knowledge, structure and actions of the MECIT. Darrell (2003: 61 ) and Reynolds (2002: 87) emphasise the importance of ensuring that all protocols and policies in relation to major emergency communication plans, instructions, routines and exercises are revised without delay. This allows the MECIT to recommence effective preparation, planning and training for dealing with future major emergencies and to expedite the revision process before valuable knowledge and experience is forgotten. The use of a broad-based consultative process should assist the MECIT in the revision process. This ensures that as much knowledge and experience as possible, both 'expert' and 'non-expert' can be gathered and assessed, and then made available to rectify any weaknesses in the major emergency communication strategic process. 
Once the revision stage has been completed it is necessary to implement the changes as quickly as possible. This involves withdrawing out-of-date communication strategies, plans and instruction manuals; issuing new instructions and protocols; and communicating information of these changes to all members of the lead response organisation, other major emergency response organisations, and any other interested parties. Coombs (1999: 146) recommends that stakeholders should also be informed of significant changes that have been implemented. Reynolds (2002: 87) emphasises that changes should be institutionalised through appropriate communication training and exercising.

\subsubsection{Institutional memory}

According to Turner (1997: 1), many major emergencies possess common characteristics that can be identified and provide a basis for the provision of experiential learning for dealing with similar situations in the future. Newsom, Turk and Kruckeberg (1996: 544) remark that direct experience with a major emergency, 'teaches more than even the best scenario ever could'. Reynolds and Seeger (2005: 45) emphasise the importance of documenting, formalising and communicating lessons learned from major emergency situations. Norman, Stuart-Black and Coles (2006: 17), consider experiential learning a cyclical process that should be continuous throughout all stages of a major emergency, but is of particular relevance during the post-crisis stage. The key phases of a learning cycle in relation to strategic major emergency communication management include many of the functions described earlier in the evaluation, revision and implementation process. Figure 8.2 (over), which is based on the learning cycle model introduced by Norman, Stuart-Black and Coles (2006: 17), graphically depicts the key elements that need to be considered in the learning process for major emergency communication:- 
Figure 8.2: Major emergency communication learning cycle

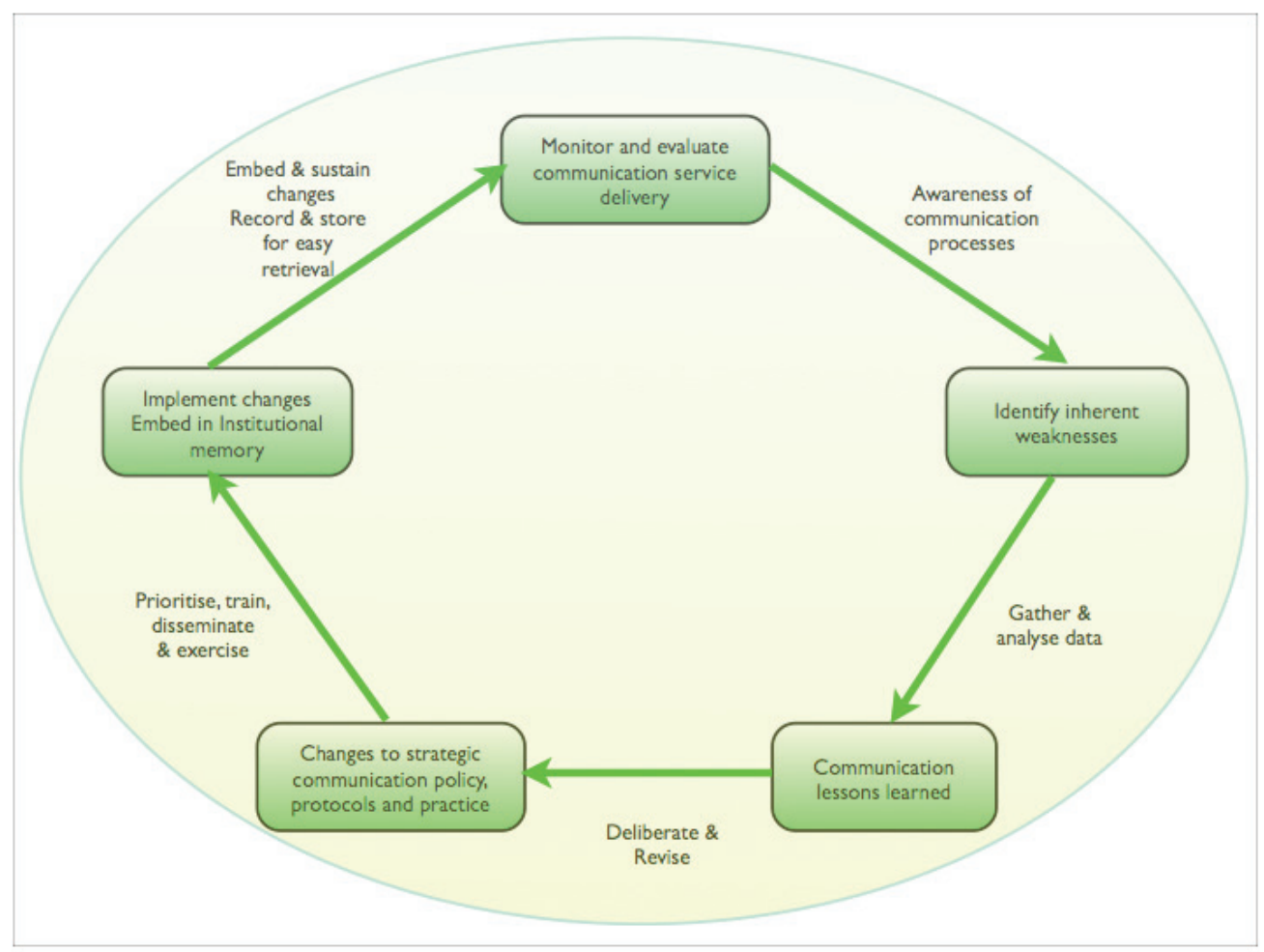

Source: (Based on Norman, Stuart-Black and Coles 2006: 17)

This model describes how information concerning inherent weaknesses in the strategic communication process should be gathered and analysed in the post-crisis stage of a major emergency. The analysis can provide important lessons in relation to improving major emergency communication policy, protocols and best-practice. These lessons should become embedded within the lead response organisation's institutional memory, through effective documentation, communication, training and exercising.

Toft (1992: 48) introduced the term 'isomorphism' to refer to the process of learning from past experiences. Some theorists such as Reason (1990: 214) and Kirkwood (1999: 33) believe that lessons learned from past major emergencies may not be applicable to other forms of major emergencies and may merely mislead responders. However, other theorists such as Toft (1992: 48) support the use of retrospective analysis of major emergency communication for the purposes of learning lessons for the future. However, according to Toft and Reynolds (2004: 88), these lessons need to be learned before key people leave organisations 'and take the lessons with them'. 
In the recording of lessons learned, Norman, Stuart-Black and Coles (2006: 17) discuss the importance of 'sense-making', where contributors to the process of institutional memory need to have a thorough understanding of 'the true nature of their previous experiences' and how this can contribute to future situations. They continue, '...it is far more difficult for effective learning to take place if the initial understanding of what has occurred is seriously flawed' (ibid).

Coombs (1999: 144) considers the effective recording and storage of major emergency communication information and lessons learned as being a key element of institutional memory, stating:

Storage is more than recording information; it also involves rating the quality of information. Key crisis information should be rated for accuracy and comprehensiveness when it is stored. To be of use, crisis information should be easy to retrieve. Retrieval involves being able to quickly search and locate specific details when required (ibid: 145).

Elliot, Smith and McGuinness (2000: 17) identify a number of potential barriers that may hinder the MECIT and other response organisations from learning useful lessons following a major emergency. These barriers include a lack of corporate responsibility, mistrust, rigidity of core beliefs, pre-conceived values and assumptions, a focus on single-loop learning, a failure to address the quality of decision-making, centrality of expertise, a disregard of outside opinion and expertise, peripheral inquiry, and the projection of blame, including 'scape-goating'.

\subsection{Link to pre-crisis stage}

Once the major emergency has passed, the lead response organisation should immediately begin to monitor and track the events and factors that are believed to have contributed to the negative situation. As with the major emergency early warning process, the MECIT once again can play a significant role in this regard. This important process assists the lead response organisation in preventing or preparing for, a recurrence of the primary major emergency and ensures that a secondary negative situation does not arise. Information-gathering teams working as part of, or closely with, the MECIT should be tasked with coordinating this important 
role. Ogrizek and Guillery (1999: 88) describe the monitoring and tracking process as switching from 'crisis unit to crisis watch'. They highlight the importance of this function as a large number of major emergencies reveal advance signals that can be readily detected and remedial actions taken. All of the information-gathering techniques described in Chapters 6 and 7 can be employed once again in the monitoring and tracking process. While a temptation may exist to disband elements of the MECIT once the major emergency has passed, the lead response organisation must ensure that the important major emergency monitoring and tracking function is maintained. Ongoing comprehensive major emergency information needs to be gathered, evaluated and disseminated to senior decision makers, together with the passing of relevant information to stakeholders, affected communities and the media.

The monitoring and tracking function logically links the post-crisis strategic communication management processes to the next stage in the major emergency life cycle, the pre-crisis stage. Reynolds and Seeger (2005: 50) consider this link to be extremely important in order to ensure that strategic major emergency communication management is an ongoing process. A link of this kind allows the MECIT of the lead response organisation to use accurate up-to-date information, together with a revised and improved communication strategy, based on lessons learned, in order to begin preparation and planning for communicating to stakeholders and publics for the next major emergency situation.

The post-crisis link to the pre-crisis stage also allows the MECIT to continue to rebuild and reinforce a positive corporate identity for the lead response organisation, thereby improving levels of public trust and credibility. Ulmer, Seeger and Sellnow (2007: 134) describe how this rebuilding of trust and credibility can be achieved through a process termed 'a discourse of renewal' that focuses on an instinctive, provisional, often value-based response to major emergency situations. This link highlights the ongoing and cyclical nature of the major emergency strategic communication process as already described in detail in Section 2.4.

In the next chapter, it is shown how three leading countries, the United States, the United Kingdom, and Sweden, utilise many of the strategic communication principles 
and strategic-tactical steps, described throughout the previous four chapters, in their day-to-day management of major emergency communication. 


\section{STRATEGIC COMMUNICATION MANAGEMENT IN THE UK, USA AND SWEDEN}

\subsection{Introduction}

During the past decade a number of countries have evolved as world leaders in the field of major emergency communication. This is due in no small way to the considerable resources and effort that these countries have committed to the constant improvement of their major emergency communication protocols, procedures and skills. These countries include the United States, the United Kingdom, Sweden, Australia, Canada, and Germany. Some other states, including Ireland, are slowly realising the importance of reconsidering their approach in the light of best-practice. Of great benefit in this respect is the various major emergency communication-related literature that the leading countries have been producing since 2000 .

Prior to analysing how the Irish government approaches the major emergency strategic communication function, it is fruitful to analyse the approach adopted by some leading countries in this field. The United States is a useful case study due to the significant time and resources that it invests in major emergency strategic communication, through the Federal Emergency Management Agency (FEMA), and also due to the many high-profile major emergencies that it has been obliged to deal with since 2001. The United Kingdom has been chosen as a result of its historical links to Ireland, particularly in public administration. Sweden is chosen because of its pro-activity in the field of major emergency communication, particularly in terms of producing relevant literature of a high quality.

Consideration of practice in these three countries will include data collated from a detailed review of available official literature, including reports, manuals, guidance materials, websites and any relevant legislation. This data is supported by information gleaned from questionnaires, completed by senior communication practitioners within the most appropriate government agency in each case. As will be seen from the results, no remarkable difference exists between the management of major emergency 
communication by the three countries examined. If anything, there is a noticeable convergence of the practices of the three countries - as is evidenced by a detailed examination of their official major emergency literature and confirmed by the international participants.

\subsection{Major emergency management}

\subsubsection{The United States}

Since the passing of the first piece of disaster legislation in 1803, in response to a significant urban fire in New Hampshire, the US government has committed considerable resources to major emergency management. Today the US is considered one of the foremost countries in emergency planning and response. A proactive approach to this discipline has been considered necessary, as the country is prone to, and often experiences, many diverse categories of major emergency situations. Examples include high profile events such as a substantial earthquake in San Francisco in 1989, the high-profile terrorist attacks in New York in 2001, Hurricane Katrina in 2005, and major forest fires in California in 2007.

Up to the late 1970s, the US government adopted a somewhat fragmented approach to major emergency management. However, following a number of poorly managed disasters, previously independent emergency response administrations and the US Civil Defense were then absorbed into a newly established Federal Emergency Management Agency (FEMA), in order to improve the response effort. In the aftermath of the 9/11 attacks in 2001, it was realised that the revised structures were not sufficient and that additional coordination measures would be required to integrate separate federal response plans into a single, all-discipline, national incident management plan. Consequently, in March 2003 a new Department of Homeland Security (DHS) was developed in order to implement these additional coordination measures (Drabek and Hoetmer 1991: 6). It employs 180,000 personnel. As part of this reorganisation, FEMA was absorbed into the DHS together with 22 disparate federal agencies. This initiative was designed to bring a coordinated approach, with a single line of authority, to national security, major emergencies and disasters (FEMA 
2008(a); DHS 2008). To provide voluntary, community assistance to the response effort, a volunteer Citizen Corps programme has been established since 2001. This is coordinated by the DHS but administered locally. The Citizen Corps helps to prepare the US population for major emergency response through public education, training and outreach and includes a community emergency response team, a fire corps, a neighbourhood watch and a medical reserve corps (Citizen Corps 2008).

FEMA is responsible for coordinating the response to any disaster that overwhelms local and state resources. The agency has an annual budget of approximately $\$ 5 \mathrm{bn}$ and 2,600 full time employees in ten regional offices. Its primary mission, within the DHS, is to reduce loss of life and property and to protect more than 300 million US citizens from all hazards, including natural disasters, acts of terrorism and other manmade disasters. It aims to achieve this by leading and supporting the United States in a risk-based, comprehensive emergency management system of preparedness, protection, response, recovery and mitigation (FEMA 2008(a)).

In relation to major emergency management, the US federal policy for response across all of the administrations is based on devolved responsibility at the lowest level possible, either local or state, through the use of an 'incident command system' (ICS) or 'unified command system' (UCS). These systems propose that all resources in a jurisdiction are made available to respond to a major emergency effort and that the structure should have the ability to expand to encompass other agencies and jurisdictions as they arrive at a major emergency scene (Ridge 2004(a): 7; Perry 2003: 405). The systems also specify that once local resources are exhausted, State and Federal resources can be requested through FEMA. Both response systems are supported by a comprehensive coordinating framework, the National Incident Management System (NIMS) (Ridge 2004(a)), which is put into effect through the all-disciplines, all-hazards, National Response Plan (NRP) (Ridge 2004(b)). Major emergency management in the US is on a statutory basis. The US government introduced major emergency legislation through the Robert T. Stafford Disaster Relief and Emergency Assistance Act, PL 100-707, signed into law November $23^{\text {rd }}$, 1988. This Act constitutes the statutory authority for most Federal disaster response activities especially as they pertain to the DHS and FEMA. This Act is supported by the 2003 Homeland Security Presidential Directive 5 (HSPD-5). This provides the 
necessary legislation for compliance by all US departments and agencies during a major emergency situation (Townsend 2006: 12). For example in relation to training and exercising, through Homeland Security Presidential Directive (HSPD)-5, the Department of Homeland Security requires all states, territories, tribal entities and local governments to undertake and complete annual national incident management systems (NIMS) compliance activities in order to achieve preparedness goals (NPG) for the United States.

\subsubsection{The United Kingdom}

Following a series of German air raids on its mainland during World War I, the UK government was obliged to undertake one of its earliest attempts at coordinated major emergency planning. A little over three decades later, fearing a nuclear attack on the UK at the start of the so called 'Cold War', more formalised planning arrangements were put in place through the UK Civil Defence Act 1948, which 'remained the hallmark of UK emergency management up to the 1980s' (Hills 1994: 67). Major emergency management was given little consideration until two detailed reviews were conducted in 1989 and 1991. These reviews came about following a number of high-profile incidents in which the response was considered unsatisfactory. The reviews proposed that major emergency planning and response should become less centralised and take place at a local level only, with support offered by the appropriate lead government department (LGD) only where necessary (Hills 1994: 67). Many of the resultant changes were 'loudly criticised' (Handmer and Parker 1991: 304) and many government departments felt 'constrained' (Beckett 2000: 1.16). These concerns were borne out during severe flooding in 2000 and a 'foot-andmouth' outbreak in 2001. Consequently, the UK government was obliged to make further changes and introduced the Civil Contingencies Act (HM Government 2004) to do so.

The Civil Contingencies Act focuses on coordinating emergency planning at both central government level, through the Cabinet Office, and at local levels in concert. It also removes all outdated legislation, re-defines emergencies, clearly identifies the roles and responsibilities of all participatory organisations and individuals throughout all stages, and introduces a mandatory regime for responders (O'Brien and Read 
2005: 358). The 2004 Act is supported by the earlier 2003 Capabilities Programme, which was designed to ensure that a robust infrastructure of response is in place to deal rapidly and effectively with major emergency situations in terms of structural, functional and essential services (Cabinet Office 2005(a): 14; UK Resilience 2007). This infrastructure was lacking in previous major emergency legislation. Among other things, the Civil Contingencies Act legislates for the assessment of major emergency preparedness, which is undertaken through its performance assessment frameworks. These assessments are overseen by performance assessment bodies, under the supervision of the Civil Contingencies Secretariat.

The focal point for emergencies requiring central government engagement is Cabinet Office Briefing Room 'A' (COBRA), where the Prime Minister often chairs interdepartmental co-ordination meetings (Cabinet Office 2005(b): 2). Lead government departments are pre-identified in the Act. In the event of a major emergency, the Prime Minister will task one lead government department (LGD) with coordinating the overall response effort across the nine regions and the devolved administrations, supported by regional resilience forums and regional resilience teams. The 2004 CCA framework allows for the coordination of inter-agency response at three management tiers, the strategic (gold), the tactical (silver) and the operational (bronze) levels.

\subsubsection{Sweden}

According to Nordlund (1994: 5), the Swedish authorities were 'challenged

significantly' following the nuclear accident in Chernobyl in 1986 and 'were found to be completely unprepared for such a major emergency'. According to 't Hart, Heyse and Boin (2001: 181), the Swedish authorities, press and general public, '.... are just waking up to the notion of emergency management and all it entails...to break the widespread assumption that disasters cannot happen here'.

Since 9/11, much attention has been given to 'potential acts of terrorism with special reference to the use of chemical, biological, nuclear/radiological (CBNR) threats' (Kulling and Holst 2004: 185). The Swedish government has introduced some important initiatives to improve its preparedness and response capabilities, particularly in the field of communication. Responsibility for so called 'severe 
peacetime emergencies' rests with the Swedish Ministry of Defence (2008). Sweden adopts a 'Total Defence' strategy, which means that it places a high value on preparing for peacetime and wartime major incidents involving input from both military and civilian resources (Kulling and Holst 2004: 185; Swedish Ministry of Defence 2008). Central to Sweden's longer-term major emergency strategy is a 1999 report entitled $A$ changing world, presented by a multi-party Swedish defence commission to the Minister of Defence. This report aims to update Sweden's defence policy to 2011, especially in relation to support for peacetime emergencies (Swedish Defence Commission 1999: 3).

According to Eksborg (2004), the new crisis management system in Sweden is based on a 'bottom-up perspective' with clearly defined roles for all. The 289 Swedish municipalities have local responsibility during a major emergency. Their roles include drawing up pre-crisis risk and vulnerability analyses, conducting major emergency training and exercises, and ensuring clear lines of communication to both regional and central level, before, during and after an emergency situation (Swedish Ministry of Defence 2008). Regional major emergency preparedness and response in Sweden is the responsibility of county administrative boards. These boards are supported in their work by joint central disaster committees, drawn from the 21 regional county councils throughout the country. The role of the county administrative boards in major emergency management includes supporting the municipalities and affiliate authorities; overseeing major emergency planning; and prioritising, distributing and guiding central government resources to the individual municipalities. This is particularly important where the major emergency spans a number of individual municipalities (ibid.).

Supporting and coordinating all of the bodies and authorities responsible for emergency management is the Swedish Emergency Management Agency (SEMA), which was established in 2002. Under the auspices of the Ministry of Defence, SEMA is located within one of the Ministry's five departments, the Department for Civil Defence Coordination (CIV). SEMA's principal role is 'to coordinate and integrate the new crisis management structure and to act as a driving force in developments in this area' (Eksborg 2004). Significantly, SEMA is also tasked with coordinating resources and allocating central funding to the relevant authorities that 
are tasked with managing a major emergency situation (Kulling and Holst 2004: 185; Swedish Ministry of Defence 2008). However, SEMA does not have an operational role and does not take over responsibility from municipalities, county councils or other authorities. Neither does SEMA have powers to force other authorities to conduct planning or exercises. However, the Swedish government is reviewing the oversight powers of SEMA currently and significant new powers are expected for the authority in 2008 (Modh 2007: personal communication).

\subsection{Major emergency strategic communication}

\subsubsection{The United States}

For many years the US government has been at the forefront of major emergency risk and communication research, planning and development. Again, the coordination of all major emergency communication efforts in the US is vested in FEMA through the DHS (Ridge 2004(b): Pub 1). Throughout much of its official major emergency related literature, the US government accords considerable importance to the management and planning of communication and information. According to Ridge (2004(a): 28):

Having systems and protocols for communicating timely and accurate information to the public and to fellow responders are critical during crisis or emergency situations.

Communicating effectively with both internal and external stakeholders is stipulated as a strategic objective by FEMA (Allbaugh 2003: 40).

As part of its strategic communication effort, the DHS, through FEMA's public affairs headquarters, provides the US public with diverse, interesting and useful major emergency preparedness and resilience information. An example of this is the dedicated, user-friendly web-site portal (FEMA 2008(a)), where FEMA posts accessible information, both in English and Spanish, on how best to prepare for a wide range of potentially disastrous situations. FEMA has undertaken a number of other useful communication initiatives, including the publication of a personal preparedness guide entitled Are you ready? an in-depth guide to citizen preparedness 
(FEMA 2004); a similar DVD entitled Getting ready for disasters (FEMA 2006); a family centred web-site, Ready America (DHS 2007); and a dedicated web-site designed to prepare and fortify children for dealing with major emergencies, FEMA for kids (FEMA 2008(b)). Separate to FEMA's efforts at major emergency communication preparedness, the Centers for Disease Control (CDC), which is the primary federal agency with responsibility for public health issues, has produced an extremely useful handbook that provides both strategic and functional communication advice (Reynolds 2002).

Despite the recent and expensive overhaul of major emergency structures in the US, FEMA has received much criticism in recent times for its management of major emergency situations, including its communication efforts. For example, its performance at the time of Hurricane Katrina was considered unresponsive (Townsend 2006: 60). FEMA was also criticised for being duplicitous when it arranged for staff to pose as journalists and ask questions during a news conference, in order to enhance its reputation at the time of the 2007 California forest fires (Lipton 2007: 2).

\subsubsection{The United Kingdom}

Responsibility for the major emergency communication function in the UK, including warning and informing the public, is vested in the Cabinet Office, through the Government Communication Network (GCN) (Cabinet Office 2005(a): 18). According to the UK government, effective major emergency communication must exist in order correctly to pass reliable information, without delay, 'to those who need to know...including the public' (Cabinet Office 2005(b): 4). The UK government, through GCN, defines major emergency communication as, '...building relationships with others, listening and understanding them, and conveying thoughts and messages clearly and congruently' (UK Resilience 2007: 2.3). The UK government has been quite active over the past decade in its attempts at communicating with the public on issues related to major emergency preparedness and response. For example, in 2004 it distributed a 22-page, multi-lingual, practical guidance booklet to all 25 million households in the UK. This initiative was augmented by a series of high-profile radio and TV advertisements intended to generate interest and raise awareness. In terms of 
providing communication management guidance for major emergency situations, the two key documents that support the 2004 Civil Contingencies Act, Emergency preparedness (HM Government 2005(a)), and Response and recovery (HM government 2005(b)) offer some extremely useful advice to major emergency communication practitioners. Additionally, there are a number of useful portals such as Preparing for emergencies (HM Government 2006) and Local response gateway (CCS 2007).

In terms of media management efforts, important UK government initiatives have been undertaken, including the establishment of regional media emergency forums. At these forums, senior UK response personnel and media practitioners can discuss, plan and agree in principle on the media's role following a disaster (UK Resilience 2005: 58). In addition, the GCN and the BBC have collaborated to produce Connecting in a Crisis (BBC 2008(b)). Other initiatives of the GCN include the News Co-ordination Centre, which can provide multi-skilled expertise to support and strengthen the lead responders media-handling and communication efforts for the duration of a major emergency. Another communication initiative involves the Government News Network providing media management skills and advice to the nine response regions.

\subsubsection{Sweden}

The Swedish Emergency Management Agency (SEMA) fulfils an important function in the development, improvement and coordination of the major emergency communication and information function across all of the Swedish response agencies. Its role in this regard includes the collection of essential information; advice in areas such as crisis communication, strategic communication analyses and research; conducting post-crisis stage communication evaluation; drawing up proposals for mandatory communication training and exercises and overseeing its implementation to a very high standard. Closely supporting SEMA in the area of communication is the National Board of Psychological Defence: Styrelsenfor Psykologiskt Forsvar (SPF 2007). The latter is a central authority, the primary role of which is to offer guidance and advice to government ministries, authorities, response agencies and the public regarding how best to optimise relations with media organisations during times 
of major emergencies. It is also involved in conducting critical media research and organising important media related seminars. Working closely with SEMA, SPF ensures government response authorities, media outlets and society are well prepared to deal with major emergency situations.

Over the past twenty years both SEMA and SPF have been very active in producing some very useful major emergency communication-related literature for members of the public and communication practitioners. Much of this information is available in English. Amongst the most useful are texts produced by Nohrsetdt (2000), Nordlund (1994), Darrell (2003) and Modh (2003). Additionally SEMA has a dedicated website through which the Swedish government can raise awareness of major emergency issues amongst the general public (SEMA 2007). Other communication initiatives undertaken by the Swedish government include the establishment of 4,500 emergency warning transmitters distributed throughout the country and a citizen-to-responder alarm system (SOS Alarm 2007: 4).

\subsection{Comparative analysis}

\subsubsection{Strategic communication management and planning}

Strategically managing the major emergency communication effort is considered important by all three agencies examined. However, while the US government frequently refers to many of the key principles of strategic communication management throughout its documentation, it was difficult to find a useful overarching strategic communication framework to guide the process, particularly within FEMA's literature. The only strategic framework that is evident is the US Centers for Disease Control's excellent five-stage framework (Reynolds 2002: 7). Despite the absence of a strategic communication guidance framework, FEMA has enshrined its strategic communication planning effort within its Strategic Plan 20032008 (Allbaugh 2003). This strategic plan establishes the 'programmatic, policy and management goals' of the agency (ibid: 11). Daryl Madden, a senior public affairs manager at FEMA's headquarters, states that FEMA takes 'very seriously' the process of engaging in deliberate strategic communication planning for all three 
stages of an emergency situation (Madden 2007: Interview). He emphasises that a flexible approach is essential in communication planning and supports the use of 'scenario-planning', stating 'it allows you train like you fight so you can fight like you train'. Madden emphasises the importance of preparing communication strategies in the short-, medium, and long-term. He supports his comments by providing a score of 9.5 out of a possible ten when asked to rate the importance of strategic communication planning.

Of the three countries analysed, the UK government offers what seems to be the most comprehensive major emergency strategic communication guidance framework. This framework is designed to link seamlessly with the UK's communication planning function. The UK government emphasises the importance of approaching communication strategically and provides six insightful guiding principles in this regard (UK Resilience 2005: 5.1). In relation to major emergency communication planning, a useful seven-step communication framework is also provided (ibid: 5.3). The communication planning function also appears prominently in a number of official documents, - in particular HM Government's Emergency preparedness (2005(a): Chapter 5) and London Resilience's Strategic emergency plan (2005: Section 2). Carol McCall, at the Cabinet Office Communications (COC), believes that major emergency communication needs to be considered strategically. She comments that communication 'developed in an ad hoc or unplanned way is unlikely to be as effective in ensuring that the public are adequately warned or informed'. Additionally, she highlights the importance of planning from the short- to the longterm. She gives strategic communication planning a rating of nine out of a possible ten in terms of importance.

The SPF in Sweden recognised well over a decade ago the importance of approaching major emergency communication from a strategic viewpoint when it developed its useful three-stage Triangle drama model in the early 1990s (Nordlund, 1994). While many of the communication principles were already being discussed in other countries, Nordlund provided a coherent framework that supported and emphasised the key elements. This model remains a useful reference document and has provided the basis for many contemporary models, including those by CDC (Reynolds 2002) 
and Coombs (1999). According to Malin Modh, a senior communication practitioner at SEMA, the process of deliberate strategic communication planning for emergency situations 'is extremely important' to the Swedish government: she scores it at nine out of ten. Modh emphasises that the twelve-year strategic report, A changing world (Swedish Defence Commission 1999), focused on many aspects of major emergency preparedness. 'This initiative gave us the impetus to look at communication over the medium- to long-term' she states (Modh 2007: personal communication).

\subsubsection{Integrated communication}

All three agencies examined were asked to discuss whether the different communication functions, mentioned throughout Chapters 6, 7 and 8, are planned for and implemented in an integrated, multidisciplinary and joined-up manner. This includes functions such as media management, public awareness and information, stakeholder relationships, community outreach and victim liaison, business continuity communications, and public affairs.

Daryl Madden states in his interview that this subject is receiving considerable attention in the US after Hurricane Katrina. According to Madden:

Joining up all of the key communication functions ensures that no gaps remain in the system...gaps lead to breakdowns in information and can create big problems.

Consequently, FEMA has devised a comprehensive integrated communication framework that encompasses all of the key functions (NRT 2000: 2.1). Interestingly, FEMA has included the information management function, usually considered an operational task, within the communication framework. According to Madden, '...this demonstrates how the information and communication functions are inextricably linked'.

While the major emergency communication-related literature in the UK and Sweden deals comprehensively with many diverse elements of the communication function, there does not seem to be a comprehensive plan available that integrates all of these diverse strands involved. Additionally, unlike the US, these two countries do not 
embrace the information management function as part of the communication role. Nevertheless, both McCall from COC and Modh from SEMA describe the importance of integrating all aspects of major emergency communication. Furthermore, they both recognise considerable advantages to the development of much closer links between communication and information management. McCall believes that there is often too much emphasis placed on the 'obvious communication functions' such as media management and public information, to the detriment of other critical areas such as communicating with the families of victims.

'Communication practitioners often consider this the role of somebody else, such as social services', she says.

\subsubsection{Model of communication}

The three agencies examined were asked to describe the model of strategic major emergency communication adopted by their respective countries. Daryl Madden of FEMA believes that the US adopts an open and transparent model of communication management. This is notwithstanding the fact that he considers FEMA to be quite hierarchical and bureaucratic. This openness and transparency, Madden emphasises, occurs at all stages of the major emergency life cycle. Madden believes that the public have a right to receive information about risks that may affect them and states that this would not be curtailed for fear of worrying them. Through its public outreach facility, Madden highlights that it is reasonably important to consult the public in relation to major emergency issues.

Similarly, the UK government identifies openness, transparency, and engaging with the public, as key principles in major emergency communication (UK Resilience 2005: 2.3). According to McQuaid (1997: 4), communication should not be treated as a mere 'bolt-on' extra, or approached solely in the context of one-way provision of public information'. McCall concurs and emphasises the importance of ensuring that regular stakeholder meetings or conference calls take place with both national organisers and local stakeholders. 'Dealing with an incident cannot be effective without their inclusion' she concludes. 
The Swedish government also emphasises its open model of major emergency communication. According to Nordlund (1994: 5) "citizens in democratic societies have a right to information that concerns them'. Modh describes as 'excellent' her country's openness and transparency when it comes to major emergency matters. She too believes that public fear should not be used as a reason for withholding important information.

\subsubsection{Broad-based deliberation (meaningful engagement with publics)}

Closely related to the previous question, all three agencies were asked to discuss whether they engage in dialogue with, and seek the assistance of, their publics when making important decisions related to major emergencies.

Through the National Research Council, the US government has supported much significant research into methods of engaging publics in important decision-making, (for example Price (1989) and Stern and Fineberg (1996)). Within its strategic plan (Allbaugh 2003: 31; Ridge 2004(c): 56), the US government, through FEMA, articulates a number of core principles that clearly provide formal support for the public engagement process. The Department of Homeland Security reviewed this commitment once again following Hurricane Katrina, when they concluded that an augmented 'national dialogue on shared responsibilities and expectations for preparedness and response' was necessary and consequently must be pursued (Townsend 2006: 82). Important studies conducted within the US, such as Redefining readiness (Lasker 2004), have provided compelling evidence of the merits of engaging publics in emergency preparedness efforts. In his responses to the questionnaire, Madden rates broad-based deliberation in major emergency decisionmaking as the second most important strategic communication principle out of ten individual principles provided.

Taking a similar view as the US government, the UK GCN highlights its government's support for meaningfully engaging the public in decisions relating to major emergency planning and preparedness. GCN stipulates that all government departments and response agencies should take 'a proactive and inclusive approach to 
consultation' with stakeholders and the public on decisions related to major emergency planning (UK Resilience 2005: 2.9). GCN provides a number of principles to be followed in this regard, including 'openness and transparency' concerning major emergency information, and 'engagement with a wide range of representative groups and the public from an early stage in the decision process' (ibid.). UK GCN also offers a very useful detailed guide for practitioners intent on involving the public in major emergency decision-making (ibid: Annex D). McCall describes very clearly how:

A broad range of stakeholders, in the recent past, played an extremely valuable part in the overall management plan for dealing with public and animal health major emergencies in the UK. The impact of their views and opinions cannot be underestimated.

McCall provides an importance rating for the broad-based deliberation process of 8.5 out of a possible 10 .

The Swedish government similarly believes that "they must be willing to enter into dialogue with the outside world and to take on board new concerns' (Darrell 2003: 21). This requires building relationships with stakeholders and preparing networks or registers, thereby 'incorporating everyone who may potentially be involved in different types of emergencies' (Darrell 2003: 18; Nordlund 1994: 41). Modh confirms this sentiment in her interview and highlights the many advantages of the process. This includes tapping into community knowledge and experience, allowing the response agencies to reach better decisions, providing greater transparency in the process thus leading to better acceptance of decision by the public, and generating better trust amongst the public. She provides an importance rating of 9.5 out of a possible 10 .

Despite these positive findings, all three interviewees believe that public consultation for major emergency situations is likely to result in delays in reaching important decisions. However, they all believe that with sufficient control mechanisms these delays can be minimised. 


\subsubsection{Management-by-objectives}

The three countries were examined to ascertain whether they put in place clear measurable, attainable communication goals and objectives as part of their overall communication process. Madden rates the management-by-objectives process as the single most important aspect of a major emergency communication strategy.

Likewise, the UK GCN highlights the necessity for having 'clear aims and objectives' in relation to major emergency communication planning, in order to help them to 'achieve their end-game' (UK Resilience 2005: 47). Despite the difficulty of finding any reference to communication goal-setting in any available Swedish literature, Modh in the questionnaire states that she considers the process 'very important'. All three agencies emphasise the importance of ensuring that all departments and agencies with responsibility for dealing with major emergency situations are fully conversant with stated communication goals and objectives. The three respondents also indicate their support for ensuring that communication goals are clearly aligned with those of the overall major emergency effort. In particular, McCall echoes the sentiment of the Civil Contingencies Secretariat, that major emergency communication planning is 'not a stand-alone duty....and goals and objectives should be fully integrated into the responder's overall emergency plans' (HM Government 2005(a): 96).

\subsubsection{Senior communication practitioner involved in decision-making at the highest level}

All three countries analysed indicate that the senior major emergency communication practitioner must be involved in the decision-making function at the highest level. According to Ridge (2004(a): 28) and the NRT (2000: vi), the US government considers the public information and communication manager as a 'key staff member supporting the incident command structure'. Through the UK Civil Contingencies Act, it is emphasised that the senior major emergency communication practitioner must have '...sufficient seniority and personal authority to take decisions and command respect... and be fully involved in the strategic decision-making arrangements' (HM Government, 2005(b): 47). 
In Sweden, Darrell (2003: 17) considers it 'almost impossible' for an authority to manage a major emergency situation if the head of information and communication is 'not part of the decision-making process'. Madden and McCall confirm that the United States and United Kingdom's senior communication practitioners are involved in decision-making at the highest level. Modh states that in Sweden the senior communication practitioner may not be actively involved in the decision-making process, but has access to decision-makers at the highest level.

\subsubsection{Knowledge base - professional education and experience}

In the US, the DHS acknowledges that professional development and education programmes are essential for improving the performances of emergency management personnel at all levels, including communication practitioners (Townsend 2006: 73).

Supporting this view, Madden explains how:

Communication in a dynamic environment is critical to the overall response... and should only be attempted by trained communication professionals in order to reduce confusion.

Consequently, Madden points out that all 64 communication and information practitioners within the public affairs section of FEMA possess communicationrelated primary degrees; a further $20 \%$ have masters degrees; and $1 \%$ have achieved doctoral qualifications. He also emphasises that ongoing communication training courses are 'mandatory' for all communication practitioners.

The UK government also emphasises the importance of 'developing formal expertise in specific areas and techniques', in the form of communication researchers and additionally through stakeholder management and consultation (UK Resilience 2005: 2.6). According to the Civil Contingencies Secretariat, 'core management skills' are also identified as being extremely important (Cabinet Office 2005(b): 33). McCall of COC highlights the importance of educating communication practitioners in order to 'introduce new approaches and processes' to the major emergency communication effort. To assist in the training of major emergency responders, including communicators, the UK government established the Cabinet Office Emergency Planning College (EPC) at Easingwold. The EPC provides a range of 
multidisciplinary formalised courses for UK response agencies. Many of these courses include major emergency communication modules, together with communication and information management seminars, workshops, and off-site exercises.

According to Eksborg (2004: 5), who is the Director General of SEMA, education, experience, training and co-operation are vital to the achievement of improved crisis communication skills and to the overall ability to respond effectively. According to Modh, formal education is considered an important attribute for communication practitioners within SEMA, with half of its communication staff possessing communication-related degrees. Despite this, Modh does not attach the same importance to formal education as she does to major emergency communication experience, technical communication skills, interpersonal skills and strategic management knowledge.

\subsubsection{Major emergency communication research}

The US National Response Team (2000: 1-4), acknowledges the importance of ongoing and iterative major emergency communication research, particularly the measurement of public perceptions, attitudes, needs and likely reactions in the face of a major emergency. In the aftermath of Hurricane Katrina, the US government recognises that enhanced communication research is required in order to improve important aspects of public preparedness and messaging (Townsend 2006: 121). With the added benefit of hindsight following Hurricane Katrina, Madden considers communication research to be particularly important for the 'development of effective messages and to ensure that these messages reach target audiences.' He provides a very high score, nine out of a possible ten, for the importance of communication research in support of the overall major emergency effort. In interview, Madden states that FEMA conducts 'a condensed research process' during the crisis stage of a major emergency, principally through focus groups and community liaison officers.

In a similar way, the UK government emphasises that departments and agencies involved in major emergency management should provide evidence that 'all relevant factors, including public concerns, values and attitudes have been taken into account' 
(UK Resilience 2005: 2.5). In practice, this task may prove extremely difficult, if not impossible, to achieve. A realistic objective for communication practitioners to achieve is a representative sample of public opinion. Useful communication research such as this, according to UK Resilience, is best carried out through conduits such as focus groups, attitude surveys and ongoing consultation with stakeholders (ibid: 5.2). McCall believes that focused communications research is essential throughout all stages in order 'to be aware of the key requirements in each major emergency situation'. She provides a very high score, eight out of a possible ten, when asked to rate its importance in supporting the overall major emergency management effort. Additionally, she considers communication research important in order to establish 'a clear baseline' against which practitioners can 'measure the effectiveness of a particular communication campaign.' In-house communication practitioners conduct the majority of research in CCS, with larger projects outsourced to external agencies.

Modh also provides a very high score of nine out of a possible ten when asked to rate the importance of communication research to the overall major emergency effort. In Sweden, Modh identifies and prioritises three types of communication research conducted by SEMA: identifying the state of public opinion and levels of trust in the response agencies; determining the public's needs and concerns; and examining how the media considers the authorities' handling of the situation. Modh states that major emergency communication research in SEMA is conducted by both in-house researchers and external research agencies.

\subsubsection{Major emergency communication evaluation}

When asked to rate the importance of evaluating major emergency communication, Madden provides a score of eight out of a possible ten. According to Madden, if major emergency communication efforts are not evaluated following implementation, 'critical information will not be received by the general public and first responders when it is needed most.' He goes further stating, 'It is not just the impact of the critical information that needs to be examined but the way this [information] is passed.' Madden highlights that the evaluation process can only be effective if it is closely related to management-by-objectives activity. He also states that the evaluation process in FEMA takes place during all stages of a major emergency life 
cycle. In-house communication practitioners conduct the majority of the evaluation activity in FEMA. The US government is committed to major emergency performance evaluation, including communication, through its annual performance plan (Allbaugh 2003: 11). In Objective 1.3 of that plan, FEMA highlights the importance of 'developing a single, uniform, after-action process to capture lessons learned' (Allbaugh 2003: 13).

In a similar way, the UK government states: 'it is good professional practice' to evaluate the effectiveness of every communication and information campaign (HM Government 2005(a): 99). As with the US and Sweden, the UK GCN includes the communication evaluation process as a key element of its strategic framework. It emphasises the importance of 'setting up arrangements' for measuring communication progress, processes and achievements, identifying problems, monitoring stakeholders' mood, finding out what aspects of communication worked and what did not, assessing cost efficiencies, and deciding whether or not involving the public contributed to decision-making (UK Resilience 2005: 41). According to McCall, the ongoing evaluation of communication programmes 'is essential' for achieving success. She provides a score of eight out of a possible ten when asked to rate its importance. 'If the effects of major emergency communication programmes are not evaluated', McCall continues, 'we will never know if what we are doing is effective.' Most major emergency communication evaluation is conducted by inhouse communication practitioners, according to McCall. However, for larger projects this task is outsourced to external practitioners, she adds.

Modh also considers the evaluation of communication, both the process of communication and its impact, as being extremely important. Providing a score of eight out of a possible ten when asked to rate its importance, she believes 'evaluation is necessary to ensure that our communication is working and that the correct message is going out to the people we want as we would like it'. Modh indicates that major emergency communication evaluation is conducted at all stages, even during the busy crisis stage. SEMA utilises both in-house and external practitioners to evaluate its major emergency communication programmes. 


\subsubsection{Building trust and credibility}

Daryl Madden of FEMA highlights the importance of taking the time to build a high level of trust and credibility with both the public and the media. In the US, FEMA identifies as one of its core values the need to be 'truthful...to listen to... and treat its publics with dignity' (Allbaugh 2003: 41). According to Madden, 'trust and credibility can only be gained by positive actions... as we discovered after Hurricane Katrina, this is easily lost.' McCall also attaches considerable importance to the CCS demonstrating its trustworthiness and credibility. She also emphasises how easy it is to lose trust and the important role the can media play in this. McCall identifies a number of critical elements that are essential in building and maintaining trust and credibility with the public and media, including honesty, openness, willingness to communicate, a rapid response, and keeping promises. She believes that those involved in an operational capacity during a major emergency, such as veterinary officers and medical officers, are 'ideally placed to build trust with members of the public'. According to Darrell, SEMA recognises that the lead response authority '...must actively work to achieve a high degree of credibility - the most important concept in crisis communication' (Darrell 2003: 13).

Modh, emphasises that 'any hint of cover-up or deceit will result in an immediate loss of trust'. She states that SEMA makes significant efforts to build trust and credibility with its public. She adds, ' ...once a major emergency happens it is too late to start...as we discovered to our detriment following Chernobyl'.

\subsubsection{Integrated communication training and exercising}

The US government emphasises the training and exercising of its responders, 'across multiple levels and disciplines' as one of its critical goals for successful major emergency response (Ridge 2004(c): 17). This sentiment is supported by the comments of Madden who believes that integrating communication into all major emergency training and exercising is 'critical for success'. He states that regular integrated communication training and exercises allow communication roles and responsibilities to be understood by all. Despite this commitment, FEMA failed to involve fully and integrate the communication function into major emergency 
training and exercises. This became all too apparent in the aftermath of Hurricane Katrina. Following this major emergency, it was recommended that major emergency communication procedures and guidelines 'need to be defined, implemented, and practiced through ongoing training, simulations and exercises' (Townsend 2006: 97).

In the UK, HM Government (2005(a): 107) describes the necessity of ensuring that regular communication 'tabletop or full-scale exercises...involving the media' take place, in order to validate arrangements and to build public confidence. Additionally, the Cabinet Office (2005(a): 66) highlights the importance of ensuring that communication training and exercises are conducted jointly with the communication teams of other response organisations, and also integrated with training and exercises conducted by other operational functions. This is to familiarise all response organisations with each other's communication terminology, protocols and procedures. McCall emphasises that 'conducting stand-alone communication training has some value but does not optimise the overall preparedness effort'. According to the UK Health Protection Agency, major emergency training and exercising provides an opportunity to test, validate and assess the entire communication plan (Norman, Stuart-Black and Coles 2006: 53).

Nordlund (1994: 41) highlights the importance of the Swedish government engaging in training and exercises 'on a continual basis' to maintain a high level of preparedness. This, he emphasises, must include communication training and exercises. Echoing McCall's opinion in the United Kingdom, Sweden's Modh (2003: 24) describes the importance of major emergency communicators receiving 'joinedup' training and exercises involving local and national journalists, together with communication officers from all other potential response agencies. 


\section{STRATEGIC COMMUNICATION MANAGEMENT IN IRELAND}

\subsection{Introduction}

Large-scale emergencies rarely occur in Ireland, but it is still necessary to consider the possibility and consequences of them. The Irish government has been criticised for providing insufficient resources in order to prepare for such events. Following the events of 9/11, major emergency management received much more attention from the government in Ireland. Thereafter, a number of high level inter-departmental and agency groups were established in order to examine and improve the standard of major emergency preparedness and response in Ireland. This resulted in the production of a new strategic framework that sets out arrangements for the principal emergency services to work together in order to manage large-scale events. As will be seen in this chapter, the subject of major emergency communication was also examined. It will be shown, in the data drawn from interviews with key personnel (listed in Appendix A), that the majority of departments and agencies with responsibility for major emergencies believe a strategic approach to communication is extremely important. However, it will also be shown that the principles of strategic major emergency communication do not seem to be greatly understood, that very little central coordination of the communication planning function exists, and that major emergency communication, in some respects, is treated as a function that does not require immediate consideration.

\subsection{Context}

\subsubsection{Natural major emergencies}

Despite the fact that approximately 200 million people are estimated to be affected annually by natural disasters worldwide, representing a threefold increase between the years 1990 and 2005, Ireland is not greatly affected by major natural disasters (UNISDR 2007). According to Brendan McWilliams: 
Ireland's relatively benign and fertile climate presents us with few of the major hazards. We do not have to endure hurricanes or large tornadoes; dust storms and avalanches are foreign to us; and really disastrous floods, droughts, heat waves or blizzards do not regularly throw our lives into chaos (McWilliams 2006: 25).

Nevertheless, Ireland has suffered some natural major emergencies over the past number of centuries. One of the most significant of these, and largely unknown to the Irish public, was the so-called 'Great Freeze' that occurred between 1740 and 1742, 'in which an estimated 480,000 people may have perished' (Dickson 1997: 67). According to a recent survey, only $10 \%$ of the Irish population consider it likely that a tsunami will occur in Ireland (Department of Defence 2005(a)). However, according to Professor Mike Williams of the Geology Department, NUI Galway, Ireland 'has been hit by tsunami at least three times in the past 250 years'. Despite presenting a low-risk, he believes, a tsunami could reach Ireland 'within hours of a volcano occurring in the Canary Islands' (Siggins 2007: 5; Williams 2007: Interview). Professor John Tyrrell of University College Cork confirms that there were at least twelve identified tornadoes in Ireland in 2006, including one over Lough Neagh 'that had a track of 26 kilometres' (McWilliams 2007: 26). According to Tyrrell, 'at least one of these tornadoes measured T4-T5 on the Tornado Intensity Scale'. He went on to say 'it is likely that a tornado could occur in Ireland that could have the potential to be devastating, with very severe damage and loss of life' (Tyrrell 2007: Interview).

One category of major emergency with which some parts of Ireland, (including Fermoy and Clonmel), are very familiar is the hydro-meteorological event, or severe flooding. In a scientific statement issued in 2007 on the subject of global warming, the Royal Irish Academy warns, ‘...new parts of Ireland will become prone to flooding and existing flood- prone areas will be affected more often as a result of climate change' (McDonald 2007: 3). Furthermore, the Office of Public Works (OPW) also states, '...it is widely anticipated that changes in rainfall patterns and rises in sea levels resulting from climate change may make such flooding incidents more frequent and severe in the future' (OPW 2008). 


\subsubsection{Anthropogenic major emergencies}

As a result of the so-called 'Celtic Tiger', or economic boom, throughout the 1990s, Ireland has experienced some considerable transformations, particularly in demographic patterns and rates of industrial development. Despite a rapidly growing population, Ireland has a reasonably low overall population density of 59 people per $\mathrm{Km}^{2}$ (CIA 2007: 53). However, there is an ever- increasing shift towards urbanisation in Ireland creating clusters of high-density population (CSO 2006(a): 14). Some of these urban centres are located close to hazardous industrial and storage facilities, creating an environment for potential anthropogenic major emergencies. Currently in Ireland there are approximately 26 higher-risk Seveso sites, each posing a potential major risk to the Irish population (Tyrell 2006: Health 8). Seveso Sites are industrial establishments containing dangerous substances in quantities that pose a grave risk to the public and are listed and regulated under a 1982 EU directive. For example, a serious incident in any of the six top-tier Seveso sites in the Dublin Docklands area could result in the potential evacuation of over 20,000 people (O'Halloran 2005). On a related topic, despite the fact that Ireland has no nuclear generating or processing facilities, the country faces a significant threat from fifty-nine nuclear power plants in France and twelve in the UK, including the nuclear reprocessing facility at Sellafield (INSC 2007). According to Kenny, '... Sellafield itself remains operational and dangerous... a disaster at Sellafield would threaten both Ireland and the United Kingdom' (Kenny 2003: 1).

One category of major emergency that has received considerable attention since 2001 is a large-scale terrorist attack. Minister for Defence, and Chairman of the Government Task Force on Major Emergency Planning, Willie O'Dea TD believes 'the risk of an international terrorist strike on any target here remains low' (Brady 2006: 3). However, Superintendent Liam King of the Garda Síochána (GS) believes that a serious terrorist incident 'is not beyond the bounds of reality' (King 2007: Interview). In the past two years, Garda and Defence Forces intelligence experts have identified approximately 24 Islamic activists in Ireland, who are under constant surveillance in relation to possible terrorist activities (Reid 2005: 6). A number of high-ranking politicians, including Minister of State for Food and Horticulture, Trevor Sargent TD, believe that Ireland is 'in the line of fire' in terms of a terrorist 
attack, as a result of the government's decision to allow US troops 'to route through Shannon Airport' (Dáil Éireann November 23 ${ }^{\text {rd }}, 2004: 7$ ).

\subsection{History}

\subsubsection{Background}

While Ireland has experienced many emergency-related events such as the risks associated with the troubles in Northern Ireland and 'foot and mouth' outbreaks, O'Riordan (1992: 43) believes that 'emergency planning has never been awarded a high priority in Ireland'. Not surprisingly, therefore, the history of major emergency planning and management in Ireland has received little attention, and as a result is largely undocumented. O'Riordan (1992) in his book, Emergency planning in Ireland, provides the first comprehensive account of the evolution of major emergency management in Ireland. However, the scope of that book is quite restricted, dealing primarily with the period 1970 through to 1992. In recent times, following the government's approval of the Major Emergency Development Programme (2006-2008), a number of guidance documents are slowly becoming available.

\subsubsection{The Cold War Period}

Despite O'Riordan's assertion (11.3.1 above), the Irish government did take some positive steps in relation to major emergency preparedness prior to the 1970s, albeit in an unstructured and uncoordinated manner. For example, during the so-called 'Cold War', many countries were exercised by the threat of nuclear attacks on their territories. From a major emergency communication viewpoint, the Civil Defence (Ireland) (1961) issued a nuclear safety handbook entitled Bás beatha (11.3.3 below) to almost every household in Ireland. Following two significant events in 1962, the detonation of a 50-megaton hydrogen bomb over the island of Novaya Zemlya in the Russian Artic and the much publicised 'Cuban Missile Crisis', the head of Civil Defence in Ireland, Gearoid Mac Pharthalain warned, '...we cannot assume that 
nuclear weapons will not in a future war be exploded on our country either deliberately or accidentally' (Cusack 2004: 26).

As a consequence, the Irish government engaged in 'an initial flurry of activity' to prepare for a potential major nuclear emergency (Horgan 2001: 16). This resulted in activities such as building a concrete bunker in Custume Barracks, Athlone, equipped with telephones and telex machines from which the government could run the country. However, despite this concerted work, no comprehensive, integrated plans were prepared by central government or local authorities for responding to major emergency situations during this period.

\subsubsection{The Irish government's first significant public information effort}

In an attempt to provide approximately one million households in Ireland with useful, practical information about how best to prepare and protect themselves and their families from a nuclear incident, Dáil Éireann approved the distribution of an insightful handbook entitled Bás beatha. This handbook was also titled, Survival in a nuclear war: advice on protection in the home and on the farm. It was colloquially termed the Home protection handbook. Bás beatha provided some detailed information in a format that could be assimilated by people with a low level of literacy, which was more common in Ireland at that time than it is today. Bás beatha used cartoon type illustrations that described complex subjects like the construction of defence shields against radiological fallout in houses and barns (Civil Defence (Ireland) 1961: 19), dangers to the gene-pool when breeding from animals exposed to radiation (ibid: 32 ), and dealing with fires (ibid: 50 ). The Civil Defence also urged households to read the booklet and to store it safely for future assistance. In this regard, they suggested punching a hole in the top left-hand corner and hanging it in a safe place, 'as it could mean the difference between life and death for you and your family' (ibid: 90).

Despite its public communication through Bás beatha, the Irish government, at another level, attempted to exert a form of censorship on the media concerning matters relating to nuclear incidents. For example, in October 1960, Prionsias MacAonghusa of Radio Eireann attempted to broadcast a radio programme dealing 
with a hypothetical nuclear strike on Ireland. The Minister for Defence at the time, Kevin Boland, was unhappy with the subject matter. He contacted the Director of the radio station and directed that the programme would not be broadcast. Radio Eireann acceded to his wishes (Horgan 2002: 17).

\subsubsection{The first comprehensive major emergency plans}

It took until 1973 for the Irish government to approve any major emergency planning initiatives. The Southern Health Board produced a comprehensive major emergency plan for its region that was completed in 1974 and this was quickly replicated by the other seven health boards (O'Riordan 1992: 44). Six years later, in the aftermath of a train crash in Buttevant, Co. Cork, resulting in the deaths of seventeen people, the Minister for the Environment, Ray Burke TD, directed that all local authorities prepare emergency plans in accordance with his department's guidelines (Department of the Environment 1981). As O'Riordan (1992: 45) observes, Minister Burke's initiative was met by the Department of Health with 'a mixture of surprise and resentment', highlighting a lack of coordination amongst key departments responsible for managing major emergencies at that time. Furthermore, in 1982 there were eight individual and unconnected health board plans and thirty-one local authority plans, containing a multitude of information that was conflicting in terms of roles, responsibilities and terminology. This resulted in 'a great deal of confusion and much duplication of effort' (O'Riordan 1992: 46). Following severe blizzards, in 1982, that almost brought the country to a standstill, Taoiseach Charles J. Haughey established an Inter-Departmental Committee on Emergency Planning to examine major emergency planning and to oversee a more coordinated approach. This provided the genesis for a plan for a coordinated multi-agency response to a major emergency, which was issued in November 1984 (Department of the Environment 1984). This 1984 Framework for coordinated responses to major emergencies was the first Irish plan that provided a definition of a major emergency, outlined procedures for activating a response, detailed roles and responsibilities, and introduced organisation mechanisms such as the coordination group and the controller of operations. 
Despite its shortcomings, the 1984 framework has underpinned major emergency preparedness and response capability for the past two decades and remains the principal reference document for major emergency response up to time of writing.

\subsubsection{Approach to communication management in the 1984 Framework}

Despite its title, the Framework for coordinated responses to major emergencies of 1984 may be regarded as a comprehensive plan for response once a major emergency actualises, rather than as an overarching strategy framework. Many of the communication functions are mentioned at different stages throughout the 1984 Framework. However, no consideration was given at that time to the consolidation of these functions. There is no attempt to introduce a common major emergency language or base terminology in this document in order to assist inter-agency communication. In respect to communicating the activation of the plan, the 1984 Framework is quite vague, detailing how 'the first agency that becomes aware of the major emergency' should activate the plan (Department of the Environment 1984: 5). However, it gives no clear guidance as to the process to be undertaken, particularly in relation to contacting other response agencies. In terms of coordinating different elements of the communication function, there is passing reference in Section 6 to communication up the chain-of-command where the document mentions 'informing government departments as appropriate' (ibid: 11). Significantly, the entire plan devotes a mere seven sentences and four footnotes to the subject of managing information and dealing with the media, stating: 'the Garda shall establish an information centre at the site of the emergency for use by the other agencies' (ibid: 14). This section also details that:

Each agency shall designate an information officer...who shall act as a sole channel of information at the site, arrange interviews, publicise emergency telephone numbers, retain copies of all information obtained (ibid.).

Providing a little more detail on the subject of technical communications, the document mentions that the controller 'shall ensure that he has secure communications with all services and agencies' (ibid: 13). 
While the information provided in the 1984 Framework offers some guidance to response agencies, it leaves the interpretation of how the communication function is approached to the discretion of the response agency concerned or to the individual on the ground. This allows a multitude of communication approaches and standards. Additionally, the 1984 Framework does not provide for training or exercising of the communication functions or practitioners of major emergency management.

\subsubsection{Post-Chernobyl}

Following the major nuclear accident in Chernobyl in 1986, Ireland's capacity for early warning, dose rate monitoring and air monitoring for radioactive fallout throughout the country was discovered to be poor. The response to the Chernobyl crisis also identified a clear requirement to 'improve communications with the public...especially information on contamination levels' (Nuclear Energy Board 1987: 19). To improve the level of response to nuclear incidents, the Radiological Protection Institute of Ireland (RPII) was tasked with establishing a network of fourteen monitoring centres throughout the State (ibid.). The government also tasked the Department of Public Enterprise with producing a National Emergency Plan for Nuclear Accidents (NEPNA): this was published in 1992 (Nuclear Safety Section 1992).

In NEPNA, an outline is given as to how Ireland will respond in the event of a nuclear accident, how technical information and radioactivity monitoring data will be provided and what measures may be taken for the protection of the public in the short and long term (RPII 2007). NEPNA is now one of a number of interdependent national and local authority plans designed to deal with various categories of major emergency situations (ibid.).

\subsubsection{Major emergency management in the new century}

The next major milestone in major emergency management in Ireland occurred in March 2001, involving an animal health outbreak of so-called 'foot-and-mouth' disease in Ireland. The response by the Department of Agriculture, Fisheries and Food (DAFF), particularly in relation to its communication function, was considered 
to be of a very high standard. Following DAFF's experience during this outbreak, Ireland became a reference point for dealing with 'foot-and-mouth' disease in Europe (Guinane 2006: Interview). Over the next four years DAFF produced some extremely useful guidance documents, including an animal health contingency plan (Gaynor and McAteer 2003).

\subsubsection{The impact of $9 / 11$}

The events of 9/11 'fundamentally affected the major emergency planning agenda in Ireland' (Nolan 2002: 1). According to a Department of Defence (DoD) press statement:

It became apparent at an early stage that there was a necessity to put structures in place to improve coordination across the various existing national plans (ibid.).

As a consequence, the government established a number of high-level committees to improve major emergency preparedness in Ireland. At the most senior level a Government Task Force on Emergency Planning was established, chaired by the Minister for Defence and his officials, in order to 'harness the work of those departments and agencies with key roles in the emergency planning process' (ibid.). Comprising of ministers and senior department officials, the Task Force's principal role is to give policy and direction, coordinate and oversee the emergency planning activities of all government departments, agencies and public authorities. It promotes the best possible use of resources and compatibility between different planning requirements (Department of Defence 2007).

Supporting the work of the Task Force, an Inter-Departmental Working Group (IDWG) on Emergency Planning was also established in 2001. This group comprises officials representing government departments and public authorities with lead or principal support roles in emergency management. This group is the 'vehicle through which expertise is shared between departments and authorities on emergency planning' (Department of Defence 2004: 9). An important role of the IDWG is to provide a forum whereby inter-departmental and agency difficulties can be sorted without using up the valuable time of the Government Task Force. The Chair of the 
Inter-Departmental Working Group is the Office of Emergency Planning (OEP), established within the Department of Defence. Not possessing any statutory authority, the OEP is responsible to the Minister for Defence, through governmental Statutory Instrument 80/46/01/0002, for the coordination and oversight of emergency planning and it provides administrative back up for this work (Department of Defence 2004: 27). However, the responsibility for ensuring that emergency plans are adequately assessed, reviewed, exercised and validated 'remains with the lead minister and government department' (ibid.).

A National Security Committee (NSC) was also established in 2001. This committee is chaired by the Secretary General to the government and comprises senior representatives of the Departments of the Taoiseach, Justice, Equality and Law Reform, Foreign Affairs, and Defence. High-ranking officers from the Garda Síochána and the Defence Forces are also represented on this committee. The NSC's role is to advise the Taoiseach and the government on high-level security matters (Department of Defence 2004: 10). As will be seen later in this chapter, a number of other committees were also established around the development of a new major emergency Framework document in 2006.

Central to the debate in Ireland after 9/11 was the government's preparedness for defending against and dealing with two categories of major emergency. The first would involve a major terrorist attack, particularly that of a 'chemical, biological, radiological, or nuclear' (CBRN) nature. Following a spate of hoax anthrax spore alerts in the US in autumn 2001, the Irish Defence Forces was obliged to deal with approximately 180 false or hoax alerts, primarily associated with mail deliveries (Irish Times 2001(b): 11). The second category of major emergency risk is posed by the UK nuclear reprocessing plant at Sellafield. High profile individuals such as Ali Hewson, wife of U2 lead singer Bono, led campaigns to highlight the dangers of Sellafield creating a Chernobyl type situation in Ireland (Hill 2002: 8). The campaign culminated with the sending of more than one million protest postcards to the Prime Minister of the United Kingdom (Kenny 2003: 213).

In an attempt to assuage the fears of the public, the Irish government issued a statement in which it highlighted the existence of extensive peacetime emergency 
plans, which are 'coordinated at different levels and covering a variety of situations' (O'Connor 2001: 1). The statement went on to discuss how the 1992 National Emergency Plan for Nuclear Accidents (NEPNA) was to be reviewed in order to 'address any weaknesses which have been identified' (Irish Times 2001(c): 4). However, it took until 2004 before this review was eventually published (Nuclear Safety Section 2004). In relation to the risk of CBRN incidents, the Garda Síochána produced a guidance document for both its own members and other response agencies to deal with any threat (GS 2003).

\subsubsection{Communication response to 9/11}

The Irish government's response in the aftermath of 9/11 proved less than satisfactory. For example, on the September $26^{\text {th }}, 2001$, the Junior Minister with responsibility for nuclear safety, Joe Jacobs TD, participated in a high-profile interview on RTE radio with broadcaster Marion Finnucane. While much of the practical advice given by Jacobs was considered to be 'not off the wall' (Guinane 2006: Interview), he was not well-briefed and his handling of the interview was far from convincing, which 'both amused and alarmed listeners' (Kenny 2003: 217). Following the interview, the media, opposition parties and the public alike pilloried Jacobs for many weeks. One national newspaper reported that 'public confidence in the State's ability to respond to a nuclear emergency has been dented' (Irish Times 2001(c): 4). According to Celina Barrett, Principal Officer in the Department of the Environment, 'the damage that one man did to the credibility of major emergency management in this country is unquantifiable' (Barrett 2007: Interview). Jacobs was subsequently replaced as a Minister of State and lost his seat in the next general election.

Despite Jacob's earlier radio fiasco, the Minister for Health and Children, Micheal Martin TD, continued to state that the government was well prepared for nuclear incidents. This was despite a belief that the threat of a nuclear incident occurring 'was considered low at that time' (Guinane 2006: Interview). In what was regarded by some media commentators as something of a public relations exercise, Martin attempted to reassure the public by emphasising that sufficient stocks of iodine tablets were available at all Health Boards. These, he said, could be issued rapidly on a 
nationwide basis in the event of a major emergency. However, when investigated by journalists of The Irish Times, it was discovered that a number of Health Boards had no usable stock, as many of the tablets had passed their safe use-by-date (Fahy and Donnellan 2001: 8). This caused much embarrassment to Minister Martin and the government coming on top of the Jacobs issue. According to Barrett (2007: Interview), 'when people talk about iodine tablets, for many they summarise our approach to major emergency management'.

A NEPNA simulation exercise, conducted in November 2001, provided another public relations fiasco for the government and Minister Jacobs, who had not yet left office, was accused of being secretive (Irish Times 2001(a): 5). However, the exercise did result in a review of NEPNA (Nuclear Safety Section 1992). The review recommended that the arrangements for emergency public information and media information should be 'fundamentally reviewed and significantly strengthened' (Department of Public Enterprise 2002: 6). Following this, in March 2002, the Department of Public Enterprise posted an information leaflet nationwide 'containing a number of key points of advice about what to do in the event of nuclear fall-out' (Cusack 2002: 1). Kenny (2003: 221) describes the content of the leaflet as containing 'very limited but practical advice'. Additionally, on June $18^{\text {th }} 2002$, more than two million packets of iodine tablets, with instructions on how to use them, were distributed to households across the country.

\subsubsection{The 2006 Framework}

In 2004, the government gave approval for the development of a document that would establish comprehensive mechanisms for the coordination of major emergency management at a number of levels. This document was to replace the Framework for coordinated responses to major emergencies (Department of the Environment 1984). The Inter-Departmental Working Group tasked the review of the 1984 document to an Inter-Agency Review Working Group with a one-year time scale to produce a revised approach. The output of this review A Framework for Major Emergency Management (together with supporting appendices) was finalised and approved by government (Goverment Task Force on Emergency Planning 2006(a) and (b)). This 2006 non-statutory Framework provides a universal structure for the coordination of 
the response of the Garda Síochána, the Health Service Executive and local authorities - the Principal Response Agencies (PRA) - to prepare for, and make, a coordinated response to all categories of major emergencies, in what is commonly referred to as the 'all-hazards approach'. These include events such as fires, transport accidents, hazardous substances incidents and severe weather (DEHLG 2007).

In order to progress the major emergency agenda, the government approved a Major Emergency Development Programme (MEDP) 2006-2008 in May 2006. The limits of the MEDP were defined and outlined in a Project Initiation Document (PID) that was approved in November 2006. The PID establishes structures at three levels, national, regional and local, to support the implementation of the MEDP. In order to support the efforts of the Government Task Force on Emergency Planning and the InterDepartmental Working Group (IDWG), approval was given for the creation of a number of groups to coordinate and implement the many elements of the Framework.

At a strategic level, the National Steering Group (NSG) was established to oversee the implementation of the two-year programme, to continue to develop, maintain and update the 2006 Framework based on feedback from the response agencies, and to report on progress to the Government Task Force on Emergency Planning (DEHLG 2007). Supporting the work of the NSG is the National Working Group (NWG) that undertakes tasks and projects associated with coordinating the response of the PRA in the event of a major emergency. Central to the work of both of these groups (NSG and NWG) is the production of two separate suites of documents that serve to underpin the implementation of the 2006 Framework. The first of these is a series of guidance documents designed to provide direction to the PRA on issues such as risk assessment, preparing major emergency plans, training programmes, working with the media, and planning and staging exercises. The second suite of documents consists of inter-agency protocols that underpin multi-agency response to different categories of major emergency, including radiological and nuclear events; situations involving conventional explosives, bombs and improvised explosive devices; aviation, rail and flooding emergencies; and infectious diseases pandemics. While the PRA plans are guided by the Framework and the NWG, individual government departments are guided in their planning by an Office of Emergency Planning (OEP) document, Strategic emergency planning guidance (Department of Defence 2004) 
and an additional OEP-produced protocol designed to detail the roles and responsibilities for each lead department and other supporting departments.

Using these guidance documents, each government department is required to produce indigenous plans and inter-departmental memorandums of understanding, that will ensure optimum coordination and cooperation for emergencies that may arise. While many of the PRA plans are well advanced, the individual department plans do not seem to be as well developed. According to an independent crisis management consultant, John Brophy:

The 'blue light' agencies plans and protocols are well advanced...the departments don't seem to be stepping up to the plate (Brophy 2008: Interview).

Confusingly, there also exists a crossover of membership of some of these groups such as the NSG the NWG and the IDWG. The roles and responsibilities of these groups, particularly the NWG and the IDWG are not dissimilar and also somewhat confusing.

In order to assist the NSG and NWG to implement the Framework at regional level, Regional Steering Groups (RSG) and Regional Working Groups (RWG) were established. These groups comprise senior personnel from the Principal Response Agencies (PRA) within each of the eight regions. In order to ensure a robust link between national and regional levels, the PID also provides a mechanism whereby representatives of the NSG and NWG attend regional meetings and regular meetings take place between the chairpersons of the regional and national groups.

Within the PRA, an internal structure has been developed in order to support the implementation of the MEDP. The 2006 Framework requires each PRA to undertake a series of steps, including the production of standard major emergency plans, engaging in training and exercising and submitting to an appraisal of its level of preparedness. The Framework imposes a two-year timeframe for each PRA to have completed these steps. However, these requirements are not underpinned by legislation and no clear guidelines exist as to how the PRA are to fund these 
activities. Despite the absence of legislation, at time of writing, the PRA appear to be on course to achieve their targets.

\section{Comparison to 1984 Framework (operational aspects)}

The 2006, or 'new' Framework differs from its 1984 predecessor in a number of ways. The 1984 document was principally a comprehensive plan, while the new document is more strategically focused, adopting a five-stage systems approach to emergency management. Unlike the 1984 document, the 2006 Framework is constructed around the systems approach to major emergency management. This is an iterative approach based on five stages namely, (i) hazard analysis/risk assessment, (ii) mitigation, (iii) planning and preparedness, (iv) response and (v) recovery. Significantly, the 2006 Framework outlines a hierarchy of relationships to underpin the up-scaling of response in the event of a major emergency. This hierarchy is based on an expansion of the day-to-day activities of the PRA.

The 2006 document formalises major emergency preparedness on the basis of eight regions, unlike the older plan. The new framework introduces common language and terminology to facilitate inter-agency working, coordination and cooperation. The concept of a 'lead-agency' has been introduced which provides a clearer understanding of command and control, responsibilities. This includes clearly identifying the principal response agency that is pre-designated to lead each category of major emergency. In effect, this means that when a major emergency occurs, the relevant emergency plan is implemented with the most appropriate department or principal response agency taking the lead in its implementation. Unlike the 1984 Framework, the 2006 document highlights the need for critical aspects of preparedness such as training and exercising. According to Pat O'Riordan, deputychair of the inter-agency review-working group that produced the document, 'two key elements which are very different to the plan gone-by include the concept of appraisal and a two-year implementation period' (Heneghan 2006: 19). It is expected that each principal response agency and supporting agency will incorporate the 2006 Framework into its individual major emergency plans. Each individual plan has a two-year implementation timeframe. 


\section{Comparison to international frameworks}

The 2006 Framework compares favourably with equivalent international guidance documents. According to Brophy (2008: Interview):

The Inter-Agency Review Working Group seem to have struck a working balance with an overall approach... somewhere in the middle ground of what is regarded as current best-practice.

Examining the equivalent of the 2006 Framework document for a number of countries, including the UK, US and Australia/New Zealand (Sweden's corresponding document is not available in English), it is clear that there are some similarities. For example, in relation to the size and detail of the document, the 2006 Framework contains somewhat less detail than the guidance documents of the United Kingdom (HM Government 2005(a); HM Government 2005(b)). In comparison to the US guidance document, National incident management system (NIMS) (Ridge 2004(a)), the new Framework is less detailed in matters relating to inter-agency cooperation, but contains more comprehensive data in relation to other functions such as information management and risk analysis. However, the new Framework is approximately three times the length, providing much more detail, than that of Australia/New Zealand's Risk management: AS/NZS4360:1999 Standard (Australian Standard 1999).

In terms of structure and format, the 2006 Framework has similarities to international equivalents. For example, the Irish document provides a mechanism for coordination at all levels of major emergency management (local, regional, national and international), defines common terminology, provides a mechanism for determining a lead response agency for every situation, and provides a process for linking to national level emergency management. As is the case with the documents produced by the US and the UK, guidance is provided in relation to important functions such as hazard analysis and risk assessment, mitigation, training and exercising, command and control, information management systems, dealing with casualties and survivors and working with voluntary agencies. Additionally, Ireland follows the lead of the other countries by providing a number of supporting guidance documents and protocols. While many of these supporting documents are still in development stage at time of writing, they are likely to prove essential as the new Framework provides 
limited detail on some important areas, including the functions of business continuity management $(\mathrm{BCM})$, building community resilience and communication.

A number of other noticeable deficiencies are evident in the 2006 Framework. For example, there is no clear reference as to the source of funding for each principal response agency in order to implement the different aspects of the Framework. Moreover, the new Framework makes no provision for the coordination of resources between regional principal response agencies, thereby creating the possibility of either gaps or duplication. Similarly, there is an absence of direction within the Framework as regards the prioritisation of spending within individual agency major emergency budgets. For example, in the course of the interviews it was reported that one county manager indicated that he did not want any risks recorded in the risk assessment phase that would 'cost his county too much money' in mitigation efforts ${ }^{1}$.

\section{The approach to communication within the 2006 Framework}

The authors of the 2006 Framework acknowledge the importance of, and deal with, many aspects of the communication function throughout the main document and within the supporting appendix. Without doubt, greater emphasis has been placed on communication and information management in the new framework than in the 1984 document.

According to the 2006 Framework, the Chair of the local coordination centre, established by the lead PRA, is responsible for "coordinating the provision of information to the public' during a major emergency (Government Task Force on Emergency Planning 2006(a): 67). In Section 5.4.7 of the new Framework, reference is made to the importance of "providing timely and accurate information to the public', together with the merits of establishing helplines, websites, and public information notices 'in order to disseminate important messages' (ibid.). Unlike the 1984 Framework, the 2006 document acknowledges the necessity of managing public perception as well as dealing with actual dangers throughout a major emergency (ibid.).

\footnotetext{
${ }^{1}$ The source of this comment does not wish to be identified.
} 
In terms of dealing with the media, the 2006 Framework provides some useful guidance. For example, it stipulates that the lead agency should establish a media centre at or near the site of the emergency, for use by each of the principal response agencies in dealing with the media once a major emergency occurs. Emphasis is placed on the nomination of a lead media liaison officer (MLO) both at the emergency site and at the local coordination centre. This lead MLO should coordinate the actions of all other response agency media liaison officers at both locations. Clearly stressed more than once is the importance of not issuing any statements to the media prior to receiving clearance from the on-site co-ordinator at the major emergency site, and the Chair of the local coordination group at the local coordination centre (ibid: 68 ). The Framework suggests that regular media briefings take place in order to promulgate helpline numbers and other important public information. It also suggests that background, 'boiler-plate', information sheets should be prepared prior to a major emergency occurring in order to form the basis of 'holding statements' that can be issued in the early stages of a major emergency in order to fill an information vacuum (ibid.).

Other important communication-related functions, that have not been considered heretofore in Ireland, are dealt with in varying levels of detail within the 2006 Framework. In Section 5.4.6, an in-depth analysis is provided in relation to the 'information management cycle' (ibid: 65). According to the Framework, this is a 'stand-alone task for trained and relatively senior managers' (ibid: 66). The information management process, including a generic information management matrix, is described in even greater detail in Appendix 10 to the 2006 Framework. Guidance is provided for a number of other communication-related functions within the 2006 Framework, including promoting resilience amongst communities (ibid: 26); dealing with survivors and relatives (ibid: 80 ); staff welfare (ibid: 85); declaring an emergency (ibid: 51); public service warnings (ibid: Appendix 6); VIP visits (ibid: 82); and common terminology and language (ibid: Appendix 3). 


\subsubsection{Major emergency public information and awareness campaign 2005-2008}

In parallel to the development and implementation of the 2006 Framework document, the Office of Emergency Planning (OEP) have undertaken a public information and awareness campaign 'in order to reassure the public as well as counteract and anticipate negative perceptions on preparedness for emergency response' (Department of Defence 2005(b): 1). The department emphasises that the campaign is designed to '... bolster public confidence in the government's ability to protect its citizens...but unnecessary alarm should not be caused...the campaign is not in response to any particular threat' (ibid.). The OEP state that this campaign will 'contribute to the overall long-term public information and conditioning element of coordinated responses to major emergencies' (ibid.). This they consider necessary, as they assert that ' ${ }^{\prime} .$. media coverage has tended to trivialise the issue [major emergencies] and to highlight perceived deficiencies in government preparedness' (ibid: 2).

The first stages of the public information and awareness campaign were undertaken in 2005, with the commissioning of initial research by Millward-Brown IMS. This research was designed to ascertain how the public perceive emergency planning and the state of preparedness in Ireland. The results of this research indicate the following: Irish citizens consider storms and floods the category of major emergency that is most likely to affect them; only $10 \%$ of respondents believe that a tsunami or earthquake is likely to occur in Ireland; urban dwellers in Ireland are more concerned about the likelihood of a terrorist related incident; less than $10 \%$ of respondents consider Ireland well prepared to deal with a major emergency situation; $66 \%$ of respondents believe that the State is ill-prepared to deal with a major emergency at national level and $70 \%$ feel that it is not sufficiently prepared at community level; over $72 \%$ of respondents believe they are not properly informed about precautions to take in the event of a major emergency; and only $7 \%$ consider themselves well informed. Of this last number, $40 \%$ state they receive most major emergency information from television and radio, $25 \%$ believe that they receive it mainly through print media, and just $10 \%$ through the Internet. Surprisingly, only $25 \%$ of respondents stated they would like to receive major emergency information by direct 
mail (Department of Defence 2005(a)). Notwithstanding, the Office of Emergency Planning decided to centre its public information campaign on a handbook that was delivered to every household by direct mail in May 2008. According to Brian Spain, head of Office Emergency Planning:

This decision was taken following careful consideration of the facts and the belief that other media would not be able to deliver sufficient information, to so many people, for such a range of diverse subjects.

Spain believes that the Millward-Brown IMS research results provided much necessary guidance for the development of the handbook, which was launched in Ireland in April 2008. According to Spain, the purpose of the handbook is to provide the public with an easy-to-understand guide in relation to the different categories of major emergencies to which Ireland is exposed (Spain 2007: Interview). It also provides useful advice as to what practical steps should be taken by the public to prepare for, prevent or respond to a major emergency situation (Heneghan 2007: 107). The launch received a good deal of coverage in both print and broadcast media. Much of the coverage cynically focused on previously mentioned public relations embarrassments for the government, such as the Joe Jacobs' radio interview and the issue of iodine tablets. According to one correspondent:

The iodine tablets were an expensive public relations disaster that has forever tainted the public's faith in government crisis management (Connolly 2008: 4).

Much of the media coverage was a parody on the new booklet, such as a ten-minute sketch on RTE's Drivetime radio programme (Boucher-Hayes 2008). Despite the negative criticism, the booklet is a useful document, containing pertinent information that is easy to access and assimilate. The 40-pages booklet deals with eight different major emergency categories in turn, providing easy to follow 'what-to-do' action points. It also provides general information for families in order to prepare for a major emergency situation, such as preparing medical kits and survival bags.

Other supporting preparedness initiatives by the government included the launch, in April 2007, of a national major emergency website containing practical reference material and information on Ireland's planning, response and management 
capabilities across a wide range of emergencies (OEP 2007). At the same time, the Chairman of the Government Task Force on Emergency Planning, Minister Willie O'Dea TD, launched a new National Emergency Coordination Centre (NECC) in Dublin. The NECC is at a central location, equipped with advanced and robust communications and conference facilities, where ministers and senior officials can convene to co-ordinate the response to a major or national emergency (Coogan 2007: 1).

\subsubsection{Communication initiatives by other response agencies}

Aside from central government communication-related initiatives, a number of departments and agencies have begun to develop their own communication material, primarily for their own use in the event of a required response. In its 'foot-andmouth' operations manual (Gaynor and McAteer 2003), the Department of Agriculture, Fisheries and Food (DAFF) has given considerable thought to its approach to the public awareness and communications functions. In Chapter 8 of that manual, proactive and ongoing public awareness efforts are deemed essential (ibid: 1.1). In Chapter 9, particular emphasis is placed on stakeholder mapping, and the importance of 'developing partnerships with the public to monitor opinion and to obtain relevant information' (ibid: 2.8). Indeed in its 2005 Annual Report, this same department identified one of its strategic goals as being, '...to raise greater public interaction-awareness...to have greater interaction with professional bodies... and to launch a public awareness programme' (DAFF 2005: 15).

To a lesser extent, the communication elements of the National Pandemic Influenza Plan, produced by the Health Service Executive in January 2007, include some well thought-out communication elements (HSE 2007). In this plan, the communication strategy is given primary importance in a list of eight key elements that require implementation. It provides guidance to emergency planners, public health officials and the public in relation to positive actions that need to be taken at different stages of a pandemic influenza. Amongst the actions are familiarising all health staff with the national plan and their roles; sending out information to households via leaflets; commencing an information campaign through posters, print and broadcast media; setting up a telephone hotline; providing a list of useful home stocks to prepare; 
ensuring the ill and disabled are looked after; and establishing a detailed up-to-date website (ibid: 17).

\subsection{Findings}

The interviews conducted with key individuals from a select number of departments and agencies provide a very useful insight into major emergency planning and communication efforts, particularly in light of the 2006 Framework. It is clear that the entire subject of major emergency management is being taken very seriously. It is also apparent that significant steps have been taken develop and enhance operational plans for dealing with major emergencies. In relation to the strategic communication function, the data from the interviews indicate that planning is not as far advanced as it is in relation to the operational function and is considered by many as 'work-inprogress'. Additionally, with the exception of a small number of departments and agencies, it is clear that there exists a limited understanding of the concept of strategic communication management and how it should be approached.

\subsubsection{Major emergency management}

All departments and agencies interviewed express their satisfaction with the new major emergency framework and view it as a major step forward in relation to coordinating the entire process. Gavin Maguire, Assistant National Director in Population Health for the Health Service Executive (HSE), believes that the Framework allows departments and agencies to 'identify structures that allow them to work together in a coordinated way at national and regional level'. Brian Spain, head of the Office of Emergency Planning (OEP) emphasises:

The new Framework is a vast improvement on the 1984 document...it is better researched with more thought gone into the content before it [the inter-agency review working group] started its work.

Independent crisis management consultant, John Brophy, however, identifies 'a glaring omission' within the new Framework involving 'a clear indication of where the money is going to come from for all of the planning, training and exercising'. 
Additionally, according to Brophy, the Framework '...contains no mention of a timeline for review of the document itself...consequently any glitches with structures or procedures will be very difficult to rectify'.

When asked if the new Framework should have a statutory basis, respondents expressed different views. Arguing that such a basis is not required, Martin Heraghty, Assistant Secretary of the Department of Agriculture, Fisheries and Food (DAFF), believes 'the enabling legislation surrounding our own day-to-day remit suffices for us to carry out our major emergency response adequately'. Brian Spain of the OEP agrees with this sentiment. The Minister for Defence and Chairman of the Government Task Force on Emergency Planning, Willie O’Dea TD, states:

I tend to think that something should only be put on a statutory basis if something can be gained from it - I can't see anything to be gained from putting major emergency management on a statutory basis.

Outlining practical problems with placing the Framework on a statutory basis, Celina Barrett, Principal Officer with the Department of the Environment, Heritage, and Local Government (DEHLG) notes:

The Civil Contingencies Secretariat in the UK drafted legislation in the first instance and then ran into practical problems on the ground - so it really is a chicken and egg situation for us.

While not advocating a statutory approach to the new Framework, Gavin Maguire of the HSE would like to see a statutory basis for national crisis management in Ireland generally, which would '...clearly define the government's powers to declare a state of national emergency and provide for specific powers for the government in this circumstance'. Maguire would also like to see a statutory basis for inter-departmental planning for national emergencies with 'clear identification of who has overall interdepartmental coordination and control responsibility'. He believes that the current lead agency concept, based on goodwill, 'can only go so far in providing a basis from which we can cooperate and work together'. Agreeing with Maguire, Tony Colgan, Director of Advisory Services of the Radiological Protection Institute of Ireland (RPII), believes that major emergency management is sufficiently important to merit placing it on a statutory footing. He describes how getting 'buy in' for 
projects in the past, such as the National Emergency Plan for Nuclear Accidents (NEPNA), proved 'very hard work' and that a statutory basis 'would be very useful' in helping to achieve acceptance of particular programmes. Brophy, an independent crisis management consultant, highlights a concern that he has learned from individual members of principal response agencies. He states:

While there is no legislation in place for the Framework, PRA are obliged by law [Seveso Directive, Article 11 (111)] to train and exercise for response to Seveso emergencies. If principal response agencies have to choose to spend their budget, time and resources on the implementation of the 2006 Framework or to comply with the Seveso Directive, I think they will opt for the latter.

In relation to the oversight of major emergency management in Ireland, many departments and agencies interviewed agree that the Department of Defence (DoD), through the OEP, is well-placed for this role and also that it is doing a good job. According to Minister Willie O’Dea TD:

With the expertise that we have in both the Department [of Defence] and the Army, we are well placed....and in view of the fact that we are not one of the busiest departments - it allows us to meet regularly and review exercises.

Lieutenant General Pat Nash, former Deputy Chief of Staff (Operations) of the Defence Forces believe that the Department of Defence is an excellent choice and 'holds a big stick over the other government departments' in order to ensure results. Nash comments that Minister O'Dea is 'very proactive and is driving along the policy'. While he believes the current system works well, Superintendent Liam King, head of emergency planning with the Garda Síochána, believes there may be merit in having a more centralised oversight role 'at the apex...the focal point'. John Brophy, independent crisis management consultant agrees with King and says, 'the closer the oversight role is to the centre of government, the greater the chance there is of pushing the agenda forward'. However, Tony Boland, Assistant Secretary with DEHLG believes, 'at the end of the day if there are difficulties, they [Department of the Taoiseach] are there to be brought back in'. Eoghan O'Neachtain, Government Press Secretary, agrees with this sentiment stating: 'If it [the major emergency] is serious enough, we [Department of the Taoiseach] will take over in any case.' Derek Mooney, political advisor to Minister Willie O'Dea, does not dispute this point. 
Taking a slightly different perspective on who is really responsible for the oversight role, Celina Barrett, Principal Officer with DEHLG, believes that in effect DoD are not empowered. She believes:

In many respects the National Steering Group, which is overseen by the DEHLG, has been empowered by government to implement the new Framework and not the DoD.

Asked whether the OEP should be given additional powers of enforcement in order to implement and review major emergency training and exercises, respondents differed somewhat. Minister O’Dea says, ‘...it is something that we can look at all right [a more robust role]...but right now it is working very well. The big stick is there anyhow if it is needed'. Brian Spain is emphatic that the government decision that established the OEP meant 'it would oversee emergency planning - not take it over'. He also states, '... I can truthfully state that we have never not achieved something that we really wanted to because we didn't have enough clout'. However, Ciara McMahon of the RPII believes that the OEP are relying on the goodwill of the other departments, and 'while the goodwill exists there will be no problem'. Eoghan O’Neachtain, Government Press Secretary, believes the issue of giving the OEP a more robust role is something that should be given serious consideration. He sees merit in giving the OEP a stronger mandate '...to oblige departments and agencies to plan, prepare, train and respond and be evaluated in a standardised and efficient manner'. Supporting this view, Superintendent Liam King believes that the OEP's oversight role should be ' ...even more independent and detached than it is currently... and perhaps should be placed under the Department of the Taoiseach'. He states further that the OEP's role should be 'more robust in terms of the integrity of the auditing process' and that 'demarcation lines should be clearly defined'. John Brophy agrees with King and places particular emphasis on powers of 'independent third-party vetting of plans and exercises'. However, not all departments agree. According to Martin Heraghty of DAFF:

Speaking from our own perspective - we don't need the OEP to oblige us to carry out our programmes of training and exercising we do this in any case. 
Most of the interviewees believe that a dedicated centralised major emergency management budget, to be administered and disbursed by the OEP, would have particular merit. Minister O'Dea believes a centralised budget would 'involve a big step' but would 'certainly provide more transparency'.

In response to being asked whether or not too many committees and working groups are involved in coordinating the major emergency effort, Eoghan O'Neachtain says 'this is inevitable' given the multitude of departments and agencies involved in major emergency planning. Brian Spain concurs and remarks, 'it is not a bad thing' to have a number of expert committees examining particular issues as 'you are getting the best possible advice'. Ciara McMahon of the RPII believes that the multitude of committees provides a great opportunity for major emergency planners to meet each other, discuss important issues and share information. These meetings allow for 'gaps' in knowledge to be filled, she concludes. According to Minister O'Dea:

The people who are saying this [that there are too many committees] don't really understand it [emergency planning]...there are two committees only really.

However, Martin Heraghty disagrees and says, ' ...there might be too many committees involved in the process...perhaps one centralised focus might be a better alternative'. Maguire of the HSE states that there could be some rationalisation and an attempt to bring some clarity to individual roles. When pressed on the issue of rationalising the number of committees involved, Brian Spain conceded, 'I would never say no to any worthwhile suggestion.'

\subsubsection{Major emergency communication management}

All interviewees were asked to identify the central body that they consider responsible for providing strategic communication guidance and advice to all departments and agencies involved in the major emergency planning and preparedness efforts. The answers indicate that a certain amount of confusion and disagreement exists as to who is actually responsible. Tony Boland of the DEHLG describes how his department looks to the National Steering Group and its working 
groups for general guidance. Taking a different view, Martin Heraghty of the DAFF states:

I suppose GIS would have to be the organisation responsible. However, as with the number of committees involved in overall major emergency planning, it tends to be a little bit confusing.

Brian Spain of the OEP also believes GIS is the most appropriate establishment to provide strategic guidance and advice on communication-related issues, particularly as 'emergency management straddles all departments and they [GIS] make a good focal point'. Adding to the confusion, Eoghan O'Neachtain, Government Press Secretary, accepts that GIS are responsible for providing guidance 'when it comes to media issues and coordinating messages from government level downwards'. However, for all other communication and media guidance, he emphasises, '... it has to be the OEP'. Gavin Maguire of the HSE comments, 'we turn inwards...to our own press people' for communication guidance. Asked whether he would turn also to the GIS for guidance, Maguire says 'absolutely not...we are working in complete isolation from the rest of the system'. Providing an example, Maguire says '...when it comes to negotiating with national broadcasters about carrying critical information for an influenza pandemic... we have to do this in isolation...even though it applies to all types of major emergency situations'. Maguire has raised this issue with the Government Task Force. However, he emphasises:

It doesn't go anywhere because there is nowhere for it to go, as there is no legislative framework in place in order to designate clear roles and responsibilities.

John Brophy, independent crisis management consultant, is more emphatic when he states:

In reality the level of expert communication knowledge just doesn't exist anywhere in the current system. GIS can advise on media related issues but after that they [the Government Task Force on Emergency Planning] will really have to bring in outside experts or else start a process of rapidly training up in-house expertise.

Derek Mooney, political advisor to Minister O'Dea accepts 'this is definitely a weakness'. In an attempt to provide clarity to the issue he states: 
Ultimately it [responsibility for providing overall strategic communication guidance and advice] does have to head towards GIS. However, the difficulty is that you are pushing it towards somebody that doesn't want it. So there needs to be consensus about where it is going to end up. Right now it is the GIS...but they are not too happy with it [the role].

Looking to the future, Mooney emphasises that the role is most likely to fall to the GIS. However, he insists that this role needs to be enhanced and must involve 'a step up from what it is at the moment'.

Brian Spain of the OEP comments that 'a central repository of communication knowledge' should be available 'that all departments and agencies can tap into as required'. Both Mooney and O'Neachtain believe that the ideal location and structure for a communication knowledge repository, such as this, is an expert communication group, which should be located within the newly established National Emergency Coordination Centre (NECC). This group would then provide guidance to the other departments and agencies.

In order to ascertain whether the selected departments and agencies have a clear understanding of what constitutes strategic communication, interviewees were asked to describe, in their opinion, what makes major emergency communication strategic. This question seemed difficult to answer for many of the interviewees. Answers included, 'ensuring that communication is properly coordinated'; 'guaranteeing communication flows between all of the response agencies, and from top to bottom'; 'speaking with one voice and having 'a good sound plan'. Minister O'Dea considers 'getting the message out...for whom the message is intended...via the media or direct contact' as being a key element of strategic communication. Eoghan O'Neachtain, who has significant practical experience as a senior communication practitioner, emphasises that in order for major emergency communication to be considered strategic it must support the overall work of the government and the lead departments; it must be viewed in the short-, medium-, and long-term; and ensuring that communication decisions are not 'pulled from the back pocket...they need to be more scientifically based'. 
A number of the interviewees accept that the 2006 Framework does not deal with the longer-term aspects of communication management. Eoghan O'Neachtain believes the new Framework 'is better' than the 1984 plan, 'however it [the communication function] is not as strategically focused as some of the other operational functions'. He also believes that the government 'is going to have to be inventive' in order to achieve a long-term focus. He believes that the use of schools and a bigger involvement in voluntary organisations is essential for success, particularly to develop strong community resilience. O’Neachtain states:

The real acid test will be in about five years time, when the new Framework has been implemented and to see then if the communication campaigning continues.

Brophy agrees with O’Neachtain and emphasises that a short-term focus for communication is 'completely at odds with the actions of other countries'. Padraig McKeown, a leading independent communications practitioner, believes that the communication approach adopted by the Irish government 'has no strategic focus whatsoever'. Emphasising the extent of the 'catch-up' that is underway in relation to major emergency management generally, Heraghty believes 'a balance needs to be found' in relation to current versus longer-term aspects of public awareness and communication management. Taking a similar view, Celina Barrett of the DEHLG explains how the new Framework document is designed 'just to put the architecture in place... it can't deal with every particular issue in depth'.

Most of the interviewees believe the new 2006 Framework devotes much more attention to communication than did the 1984 document. However, there are mixed views as to whether the communication model, presented within the new Framework, provides an adequate structure for coordinating all of the disparate elements of the communication process, including information management. Many of the interviewees demonstrated a limited appreciation of some elements that need to be considered as part of the major emergency communication management effort. However, Lieutenant General Pat Nash and Superintendent Liam King are satisfied that the different elements of communication are sufficiently integrated within the new Framework. Celina Barrett concurs, highlighting that the information management system within the new Framework provides 'the coherent architecture 
around which all of the essential elements of communication are integrated'. Both Martin Heraghty and Gavin Maguire strongly disagree and believe that more thought and effort needs to go into this joining up of all of the different elements of communication.

Additionally, John Brophy highlights that a number of communication projects have been undertaken by different departments and agencies 'without any great coordination or joined-up thinking'. O'Neachtain of GIS believes that every major emergency communication project that is designed to guide other departments and agencies 'needs to come from a sound and well-coordinated basis'.

Interviewees were then asked whether the communication and information management functions should be amalgamated, under a single information officer reporting directly to the off-site coordinator. While the model proposed in the 2006 Framework document separates the information and communication management functions, the US Joint Information Center model supports the amalgamated approach (NRT 2000: 1-6) . Martin Heraghty of DAFF sees much merit to the US approach. He says, '...from our own experience of dealing with "foot-and-mouth", this is the way that communication should be managed and controlled'.

Similarly, Gavin Maguire believes that 'in a big bang major emergency, essential elements such as communication and information clearly cannot be treated separately'. Brian Spain of the OEP admits, 'I haven't given this much thought'. However, he goes on to say:

It makes sense that one person has all of the information and that they should make the critical decisions as regards which information should be released and to whom - including the media.

Eoghan O'Neachtain also agrees that information and communication management require integration in order to deal effectively with a major emergency. He comments:

This has to happen. It is like having a stool with three legs. Information comes in through communication channels. Information is then prioritised, analysed and processed in the information hub. 
Then it is sent out through communication channels. And you can see from that, both are hand in glove - inseparable. They should be managed accordingly.

Not entirely convinced, Tony Boland of the DEHLG views information management and communication management as two separate, but parallel functions. He emphasises that his own department's 'schematic' provides for a media manager who has the same status as the information manager and 'both should report into the onsite coordinator'.

All interviewees agreed that the major emergency communication manager should be part of the decision-making process at the highest level. According to O'Neachtain, 'communication will add no value unless the manager is standing right next to the coordinator'.

\subsubsection{Elements of strategic communication management}

One of the essential strategic communication elements identified throughout this dissertation is the engagement of a broad base of stakeholders in a meaningful way in order to arrive at major emergency decisions. From the interviews it is clear that the majority of the interviewees are positively disposed towards the concept. Minister O'Dea supports this principle, saying, '... absolutely...consulting with the public in advance of a major emergency is well worthwhile considering'. Clearly from this comment, the minister considers engaging with the public as merely an aspiration at this time. This point is reinforced when the minister says, 'but you couldn't do it at the moment because the lack of basic knowledge is so bad [amongst the public]'. Eoghan O'Neachtain also agrees in principle with a broad-based analytic deliberative process. He says:

I can see great value in this and I know that this is the way other countries are headed. This should become part and parcel of the way we do business for important decisions, including major emergencies.

However, O’Neachtain believes that there may be certain difficulties in achieving agreement to this process. He says, 'we have to get through a particular mindset [public service] that is not going to change overnight'. McKeown questions whether 
the necessary levels of expertise exist within departments and agencies in order to engage in public engagement in a meaningful way. At a very practical level, in preparation for influenza pandemic, Gavin Maguire of the HSE admits that they have 'not yet engaged in any significant public dialogue to date'. However, he says that future communication research with members of the public will '...impact areas such as the design, content and message on the leaflet [influenza pandemic] to be delivered to every household... and also indicate the degree to which the general population will take direction during a pandemic or other prolonged national crises'.

Martin Heraghty of DAFF also believes that by engaging in a broad-based analytic deliberative process, a mechanism is provided in order to 'find out new ways of doing things or getting useful information'. Displaying perhaps a little less enthusiasm for the public-engagement process, Brian Spain of the OEP concludes that, 'it depends on how meaningful and what type of contribution these stakeholders and publics can supply'. He also believes that it may be difficult to interest the public in engagement when the likelihood of a major emergency is low.

Interviewees generally consider the process of approaching major emergency communication strategically as being extremely important. Martin Heraghty describes how DAFF 'has learned this through experience, particularly during the "foot-andmouth" crisis'. Tony Colgan of the RPII emphasises that 'all of our successes in the technical area can be lost if our strategic communication is bad'. According to Brian Spain of the OEP:

Communication needs to be a slow burner...there needs to be an ongoing strategy. Major emergency communication cannot be viewed as a side-issue or a bolt-on... it must be planned.

Eoghan O'Neachtain emphasises that 'every other aspect of major emergency management is looked at strategically... why not communication?' O'Neachtain goes further stating:

We have learned some important lessons from not approaching communication strategically in the recent past. In some of these situations it was really finger-in-the-dam stuff...hoping that the media were going to lose interest in the issue very quickly - allowing us to get on with the business of the day. It doesn't work out like 
that though. Now we are faced with a real major emergency in the form of a major flu pandemic. If our communication is not strategically focused leading up to that - we will be in big trouble.

There was general agreement amongst the interviewees that setting clear, attainable communication goals and objectives (management-by-objectives) is also extremely important in order to achieve measurable success when engaging in communication programmes. Superintendent Liam King rates the importance of communication goalsetting at seven out of a possible ten, while Lieutenant General Nash provides a rating of eight out of ten. Without exception, all interviewees report that the alignment of communication goals with operational goals is essential for the overall success of the preparedness and response effort.

There was broad agreement amongst the interviewees that all departments and agencies should introduce a more scientific approach to their major emergency communication efforts. However, it appears that in practice the benefits of practitioners having a good level of formal communication education and experience, of engaging in communication research and evaluation, and of developing and testing key messages, may not be fully appreciated. Additionally, the degree to which a scientific approach to communication is adopted by response departments and agencies varies considerably.

Gavin Maguire of the HSE believes that communication research is extremely important, particularly in relation to his preparations for influenza pandemic. Eoghan O'Neachtain was emphatic in relation to the value of communication research. $\mathrm{He}$ asks:

If the information is not evidence-based, how reliable can it be? There is no point in guessing or making assumptions that we know how people think.

Brian Spain agrees with O'Neachtain and describes how, prior to the development of the major emergency public information campaign, he believed people would want to receive emergency planning information by the web. However, following detailed communication research this was proven '...to be wrong - it was way down on their preferred list'. Derek Mooney highlights how formal communication research was 
conducted in order to ascertain levels of knowledge and perceptions amongst the public prior to undertaking the public awareness campaign process. The minister indicated that communication research is something he will continue. However, this commitment relates specifically to public awareness. Superintendent Liam King also considers communication research important. However, he says that the Garda Síochána (GS) does not engage in any research of this kind currently in relation to major emergency planning. Similarly, Martin Heraghty understands the significance of engaging in communication research. However, he says that DAFF does not conduct any formal communication research for major emergency management.

All of the interviewees had a standard approach in terms of developing major emergency key messages. In the majority of cases, the department or agency press officer, together with a core group of major emergency planners, devise their respective messages. None of the interviewees report that they engage in formal key message testing prior to dissemination. A small number of respondents, such as those in DAFF and the HSE, say that they test the efficacy of some related advertisements. Brian Spain of the OEP states:

I believe we genuinely should test messages. In our own heads we have a certain sense of what is right and what the public want. But we should get their views for what they really want.

Eoghan O'Neachtain agrees and comments, 'I think it [testing] would ensure that the messages work.'

When it comes to evaluating the efficacy of key message development and dissemination, all interviewees believe the activity to be worthwhile. Eoghan O’Neachtain says, '... we need to evaluate messages... we may think it is a great message...but is it effective... does it achieve its desired effect?' O'Neachtain goes further, observing that evaluation should involve both message dissemination process and impact. 'If one or both are not working...then we cannot achieve our goals', he states. Spain agrees and describes how in any process 'you should be willing to learn and to admit that there are better ways of doing things'. 
Very few of the interviewees engage in the formal testing or evaluation of the efficacy of their chosen channels of communication for major emergency communication. O'Neachtain highlights the importance of this process, particulary for reaching marginalised groups such as people with disabilities and Travellers. Heraghty says that DAFF does engage in some informal research. This, he says, includes investigating the efficacy of the DAFF's channels of communication for reaching its target audience. DAFF conducts this research at large-scale events such as the annual National Ploughing Championship. Spain describes how the OEP research, but do not test or evaluate, channels of communication. McKeown believes that testing, such as this, 'is beyond the comprehension of individual departments and agencies'.

The interviewees were asked to discuss how important formal communication education and adequate experience are to major emergency communication practitioners in their day-to-day preparedness and response efforts. The majority of interviewees saw great merit in employing communication practitioners with formal communication education. However, in practice, very few of the departments and agencies choose their communication practitioners on this basis. Martin Heraghty of DAFF comments: 'We don't have the luxury of targeting and training-up our communication officers prior to selection.' Lieutenant General Pat Nash emphasises how carefully targeting selection and formally educating communication and information specialists 'is the way to go'. However, he highlights how specialising in a narrow field of expertise, such as communication, can be 'career-limiting' in organisations such as the Defence Forces. Gavin Maguire of the HSE views the selection process as a key factor. He says:

In the future, I think there is a clear need to consider targeting our communication practitioners, choosing those with specific qualifications and encouraging others to undertake strategic communication courses.

Eoghan O’Neachtain, who himself has a Masters in Communications and Cultural Studies (MACCS) from Dublin City University, believes that an academic grounding in communication is 'very important...but of itself it is of minimal value'. He 
qualifies his seeming paradoxical comment stating: 'Coupled with experience, training and practice, academic knowledge can be a great asset.'

When asked to discuss whether the government adopts an open and transparent approach with publics in relation to major emergency planning, thereby attempting to imbue a sense of trust and credibility amongst the public, all interviewees believed that levels of candour have improved. According to Martin Heraghty of DAFF:

From our experience, the best way to handle difficult issues is not to hide from them, and to give the information. However, this has to be balanced with not unduly worrying people by giving them worrying information for no good reason.

Superintendent Liam King of GS is adamant about the importance of transparency and openness when it comes to building trust and credibility. He remarks:

There is no point in deluding yourself and not telling people - they probably already know what is going on [in the area of major emergency management].

O'Neachtain notes that in relation to major emergency management, openness and transparency amongst departments and agencies 'has come from a very low baseline'. He believes that this is due in no uncertain terms to their [response departments and agencies] previous levels of preparedness, and says, '... as we have seen in the past, if the Principal Response Agencies are not prepared, the media will slaughter them'. Heraghty believes that the government have been reasonably open and transparent with the public to date. Lieutenant General Pat Nash, observes, ‘... I think it [openness and transparency] has transformed over the past 25 years...we now have a certain openness, within certain limits'. Minister O'Dea is more emphatic when he states: 'We have swept away all the secrecy ... the media now have to have access to exercises.' Eoghan O'Neachtain highlights the elements required to achieve a high level of trust and credibility amongst the public,

...being consistent and not getting caught telling fibs. Also, it is important to be perceived as an expert, experienced, reassuring - and to use a commonality of language. 
All interviewees agreed that a high level of public trust and credibility in the response agencies is extremely important. Celine Barrett states that credibility has been damaged by the Joe Jacobs' interview, mentioned earlier in this chapter. King believes that this trust can be rebuilt once integrated exercises begin in earnest. During the interview, interviewees were asked specifically to discuss whether the public is likely to trust the HSE to manage a major emergency such as an influenza pandemic, in light of the reported 'serious loss of confidence in the public health sector' (Hardiman 2007: 16). Minister O'Dea agreed that this is a problem and states:

The HSE is in very bad order and and an unpopular institution out there - there is no doubt about that and I have no hesitation in saying it... the lack of accountability and contact is frustrating us all, and the public don't like the HSE... and this is not helpful. I know that the HSE are the lead agency for dealing with a pandemic but they are only a cog in the wheel.

Most of the interviewees, including Maguire, agree with the minister's sentiment in this regard. Brophy, believes that the HSE has 'a significant obstacle to surmount' in order to 'win back the public's trust' should a pandemic situation occur.

When asked whether or not a strategic major emergency communication model, which involves communicating with communities rather than merely to communities, is likely to work in the Irish cultural context, interviewees provided mixed responses. Eoghan O'Neachtain believes that within the 'global village', the Irish psyche is 'not wholly dissimilar to that of people in other countries considered leaders in major emergency management'. Based on his experience with 'foot and mouth' internationally, Heraghty agrees with O'Neachtain and believes that many of the elements of a major emergency communication strategy 'can be applied readily in the Irish context'. In the event of a serious major emergency situation, Maguire is not convinced that all elements of a 'best-practice' communication strategy can transpose easily to the Irish context. In the event of an influenza pandemic, for example, Maguire believes that many infected people will be instructed to remain at home and not to attend acute hospitals in order to prevent cross-infection. He continues:

We can follow best strategic communication practice all we like engage with publics, develop and test messages to make sure they work. However, Ireland being Ireland, the person with the sick child 
who is instructed to remain at home is bound to know a person who knows a Garda sergeant who will make a phone call to get the child to hospital. So while the perfect communication model may work well in Sweden - it may not work equally as well in Ireland.

Brophy agrees with Maguire and states that 'a great deal of common sense needs to be applied to any communication strategy'. 


\section{CONCLUSIONS AND RECOMMENDATIONS}

\subsection{Introduction}

The purpose of this study has been to explore the strategic management of major emergency communication, with particular emphasis on how the Irish government approaches this important discipline. In order to examine thoroughly the principal research question it was necessary to scrutinise a number of analogous and interrelated subjects such as developments in the theory and practice of major emergency management both in Ireland and internationally. Semi-structured interviews with key personnel in both Ireland and a number of leading other countries were undertaken. As mentioned in Chapter 1, leading countries in this regard are those that are considered to be innovators in the development of major emergency theory and practice. These countries engage in best international communication practice, as discussed in Section 1.2, and include countries such as the United States, the United Kingdom, Sweden, Canada, and Australia. Major emergency literature has also been reviewed.

It is possible to draw a number of conclusions. For ease of presentation, these conclusions are presented in four separate categories: major emergency management internationally, major emergency management in Ireland, major emergency communication management internationally, and major emergency communication management in Ireland.

Based on the conclusions, the final section of Chapter 11 advances two recommendations for government departments and agencies with responsibility for major emergency management in Ireland. These recommendations have been developed taking into account best-practice and are offered in order to provide a focus for the future development of major emergency communication theory and practice. 


\subsection{Major emergency management}

\subsubsection{Theory and practice}

It is evident that there have been many advances in the theory and the practice of major emergency management over the past thirty years, with significant changes occurring in the past five years. What is evident from an examination of international major emergency documentation, supported by data provided by interviewees, is that most leading countries have adopted a somewhat similar model of major emergency management that involves a continuous cycle of activity and that employs a systems management perspective. The research also highlights the fact that leading countries approach major emergency management largely from a strategic perspective. As discussed in Chapter 4, this strategic approach mirrors how large corporations have learned to conduct business and survive in uncertain environments. Adopting a similar methodology, it can be concluded that countries engaging in best-practice set clear major emergency goals and objectives, undertake specific research, produce comprehensive plans and continuously evaluate, review and improve their preparedness and response performances. Additionally, leading countries are now treating major emergency management as a field of science rather than a mere operational task. This is evidenced by the quality of major emergency research data and literature available, and also by the proliferation of major emergency related third level courses in many countries, including the MSc in Emergency Management in Dublin City University.

While most countries up to the late 1970s considered effective major emergency management principally in terms of having an efficient response capability, this study shows that leading countries now place equal emphasis on the planning and preparedness functions. This is evidenced by the major emergency structures that leading countries have developed and by the amount of effort afforded to preparedness, training and exercises. It is also evident that leading countries are placing considerable emphasis on managing specific risks, hazards and vulnerabilities, through prevention and mitigation measures. 
In relation to the major emergency response, it can be concluded from the data that leading countries plan this important function on the principle of devolved responsibility at the lowest level possible, with higher level coordination and support provided by well-trained major emergency agencies. Additionally, the study reveals that the sequence of steps taken by leading countries in order to manage a major emergency situation is remarkably similar. These steps are contained within welldeveloped plans that are a central element of each country's preparedness efforts. The practice of leading countries indicates that major emergency plans are thoroughly developed, continuously updated, regularly tested and flexible enough to be able to deal with unexpected issues.

\subsubsection{Revision and evaluation of major emergency structures and plans}

It can be deduced from the research data that the many of the leading countries in the field of major emergency management have undergone, or are currently in the process of undergoing, a significant overhaul of their major emergency structures, legislation, guidelines, protocols and procedures. Consequently, it can be concluded that countries engaging in best-practice continuously evaluate, and if necessary revise, all elements of their major emergency management structures, processes and procedures. This is particularly important in the aftermath of a major emergency situation when inherent systemic weaknesses are likely to be exposed. The practice of major emergency management continues to evolve and while there is a convergence of theory and practice across leading countries, it is difficult to state categorically that these processes have been optimised at the time of writing this report. There have been many developments in the process of major emergency management over the past fifty years. It is likely that these leading countries will be utilising a different methodology in fifty years time.

The research also reveals that leading countries see considerable merit in producing comprehensive, self-critical and publicly available reports, following major emergency situations, which are designed to improve the overall major emergency management effort. 


\subsubsection{Major emergency legislation}

Even though not all countries examined have major emergency legislation in existence (Sweden does not), it can be concluded from the research that dedicated, robust legislation is important in order to support and underpin the major emergency effort. Interviewees from the international agencies advance many justifications for this perspective. Foremost, in support of major emergency legislation, is that leading countries believe that no ambiguity should exist regarding the roles and responsibilities of all government departments, response organisations and individuals, involved in the major emergency effort throughout all stages. Other arguments put forward in support of robust legislation include ensuring that all departments and agencies have an unequivocal obligation to prepare, train and exercise for major emergency situations; ensuring that clear responsibility exists for the oversight function and for the review of performance and standards; and making certain that clear budgetary provision for major emergency management exists. From an analysis of the data, it can be concluded that most of countries the countries examined as part of this research have robust legislation in place to support the major emergency management function. Additionally, it can be concluded that effective mechanisms are necessary in order to ensure that the provisions of the legislation are being strictly adhered to and implemented.

In addition to putting major emergency legislation in place, the agencies examined describe the importance of producing strategic major emergency guidance documents and clear protocols in order to integrate the work of individual government departments, civil servants and all of the response agencies throughout all stages. They include protocols for international cooperation. The data from the interviews highlight that international response agencies spend a considerable amount of time developing clear major emergency protocols and guidelines, testing their effectiveness, and then disseminating all relevant information to each major emergency response organisation. There is evidence that these protocols and guidelines are put into practice on a regular basis through both training and interagency exercises. Without such an effort, it can be concluded that different departments and agencies, many of which have different methods of thinking and 
operating, are likely to find it very difficult to work together when a major emergency occurs.

\subsubsection{Major emergency oversight}

The research data confirm that countries engaging in best-practice have put in place dedicated structures in order to oversee and coordinate their major emergency efforts. The study also concludes that the oversight function for major emergency management is given more emphasis in some countries than others. For example, many countries have established dedicated government departments that have been given sole responsibility for the oversight and coordination role. Others, such as the United Kingdom, locate their oversight role at the 'very centre... a head office of government' and play a central role in planning for emergencies (Cabinet Office 2008). Consequently, it can be concluded from the research that the majority of leading countries have placed this important function as close to the centre of government as possible, with direct lines of access to the most senior decision-makers in government. It can also be concluded that by adopting such an approach, the major emergency management function is given greater priority amongst the many other important day-to-day functions of government.

\subsection{Major emergency management - Irish context}

\subsubsection{Advances in major emergency theory and practice}

From this study, it can be concluded that while the threat of a major emergency is considered low in Ireland currently, particular categories of serious incidents, requiring a significant multi-principal response agency (PRA) response, cannot be ignored and should be prepared for. These categories include a major terrorist incident; a large hydro-meteorological event; a significant storm; influenza pandemic; a large-scale animal health incident; an anthropogenic major emergency, particularly from a UK or Europe based nuclear generation or reprocessing plant, or from a Seveso II category plant in Ireland; and a major transport-related accident. As described in Section 2.4.1.2.2, adequate preparation helps to limit the amount of 
confusion once a major emergency occurs, clearly defines all roles and responsibilities for those involved in the response and allows each role to be tested during realistic training and exercises. As is happening through the roll-out of the 2006 Framework, adequate preparedness requires structures to be put in place, strategies and protocols to be developed and resources made available. However, a pragmatic approach to the subject of major emergency communication management is required in Ireland. This should take cognisance of the major emergencies that are most likely to occur and those that are not, based on past experiences; the likely impact to society; and the cost in terms of money and resources. The need for such an approach was clearly demonstrated in December 2008 when the Irish government decided to recall all pig meat products both at home and abroad, and ordered the slaughter of over 100,000 pigs. This arose following the discovery of low levels of dioxins in pork products and pig feed. This decision is estimated to have cost the country approximately $€ 180 \mathrm{~m}$ and to have risked up to 1,400 jobs (Mullooly 2008; Leahy 2008: 8). The government's decision was designed to communicate confidence in the Irish meat industry and in this case communication and operational action was swift and decisive. However, it was criticised by many as too hasty and an overreaction. While this was not a major emergency situation, it highlights the necessity for a clear cost-benefit analysis to be undertaken prior to taking significant decisions following an emergency situation.

Notwithstanding, following international trends, it can be concluded from the research data that significant advances have been made in the entire field of major emergency management in Ireland since the commencement of this study in 2004. This is evidenced by the many initiatives undertaken by the Irish government since that time and it is clear that much useful work is in train currently. In line with the practices of countries examined as part of this research, response agencies in Ireland are now treating major emergencies as cyclical events that involve a continuous cycle of planning and action. From a theoretical perspective, much emphasis is placed on important functions such as risk assessment, mitigation and risk management. Additionally, structured and comprehensive planning guidance is provided in order to allow a common, best-practice, major emergency plan to be produced by all PRA. At the time of writing, these plans are being produced by PRA throughout the country. Consequently, it can be concluded that Ireland is following best-practice in relation to 
the development and propagation of major emergency management theory. It is premature, at this stage to draw any conclusions in relation to whether the evolving methodology developed and practiced by leading countries is best suited to Ireland's needs. Additionally, it is too early to pass judgement on the implementation of this theory (the practice) as it remains a work in progress. However, it is sufficient to say that PRA remain guided by the 1984 Framework and are obliged to work within the constraints that the process offers. While the practices proposed by the 1984 document provide for a robust individual PRA response, they lack the integrated, allhazards, systems approach to major emergency management that the 2006 Framework advances.

In terms of major emergency development practice, from the research it can be concluded that the different groups such as the Government Task Force on Emergency Planning, the Inter-departmental Working Group, the National Steering Group, the National Working Group and the various regional groups, are very proactive in progressing the major emergency planning agenda. Although having very comprehensive and busy work schedules, they meet regularly in order to implement the many elements of the 2006 Framework. According to many of the interviewees, the results of the major emergency management effort are slowly becoming manifest on the ground.

The research data reveal that there are mixed views concerning the multitude of official working groups that have been established in order to progress the wider major emergency preparedness and planning effort. While some of the interviewees believe the current structures are necessary and useful, others suspect that some duplication of effort exists, that the role of each group is not absolutely clear and that there may be merit in some rationalisation. This concern relates particularly to the role of the IDWG and the NWG. It is not possible at this stage to draw a conclusion as to which school of thought has most merit. However, as major emergency management in Ireland is coming from such a low base, the input from such a diverse range of groups can only provide positive benefits. Additionally, changing the structures, as they currently exist, is likely to hinder the progression of the implementation of the 2006 Framework. 


\subsubsection{Whether the 2006 Framework reflects best-practice?}

Taking account of the fact that the methodology for major emergency management adopted by leading countries is continuously evolving, and practices that constitute best-practice currently are constantly changing, the data acquired from a study of the literature, supported by detailed interviews, provide evidence that Ireland's newly developed Framework for major emergency management reflects best-internationalpractice. From an analysis of the data, it can be discerned that the Framework, which is based on a 'systems' and an 'all-hazards' approach, compares very well with international models. As described in Chapter 10, the 2006 Framework has followed a format adopted by leading countries and embraces the 'lead-agency' concept, prescribes a common lexicon of terminology and includes guidance on many key functions such as risk assessment, information management and working with voluntary organisations. In terms of size and level of detail, the Framework has achieved a balance with a broad approach somewhere in the centre of what is regarded as current best-practice. Additionally, the research indicates that the approach taken towards the development of the document is strategically grounded. The interviewees highlight that the inter-agency review-working group that produced the document utilised many strategic principles such as setting clear goals and timeframes, engaging in research in the early stages, evaluating their output at various stages, and engaging outside expertise for particular aspects of the process.

However, a close examination of the data reveals a number of shortcomings within the 2006 Framework. For example, no clarity is provided as to what happens once the 2006 Framework is implemented in late 2008. This means that any deficiencies discovered within the Framework model will be more difficult to deal with as no time has been provided for a review and revision of the actual Framework model itself. As described earlier, leading countries constantly update and revise their major emergency approaches, theory and practice. It can be concluded that without such a mechanism being clearly stated, Ireland may be implementing and building on a substandard major emergency model.

The research also reveals that the 2006 Framework provides little detail in a number of important areas, including the functions of business continuity management 
(BCM), building community resilience and communication. Furthermore, it seems as if there are no plans in place to develop guidance documents for these particular functions. Without comprehensive guidance on these critical areas, PRA are likely to ignore these important functions entirely or else may produce their own guidance documents that bear no correlation to those of other response agencies. It can be concluded that clear guidance documents are critical in order to provide direction to all departments, agencies and organisations involved in the major emergency preparedness, planning and response effort. Additionally, in the production of major emergency plans at national, regional and local level, the 'blue light' organisations are obliged to consult one category of guidance documents, while government departments are obliged to consult another. This creates a lack of coordination in the planning process. Furthermore, until such time as the OEP has produced and disseminated its guidance protocols to each government department, it is unlikely that a fully integrated implementation of the 2006 Framework is possible. In particular, this is likely to prevent any meaningful involvement of government departments in major emergency training and exercises for the foreseeable future.

Another significant shortcoming in the 2006 Framework, identified by the study, is the lack of clarity in relation to budgetary allocation to individual departments, agencies and regions. It can be concluded from the data that this is likely to generate potential difficulties for an effective implementation of the Framework requirements. A lack of clear guidance on budgetary issues is likely to impact on the PRA's capacity to purchase any necessary response equipment, put in place any significant mitigation measures, or engage in individual or inter-agency training and exercising.

\subsubsection{Statutory status of the 2006 Framework}

As discussed in Chapter 10, the 2006 Framework was approved by government but never passed into legislation. It can be concluded from the research that this is not in line with the practice of international countries examined where many of them have their major emergency management efforts underpinned by law. It is clear that opinions differ as to whether or not major emergency management in Ireland should be placed on a statutory basis. The data highlights that a number of interviewees consider the existing enabling legislation that surrounds the day-to-day work of the 
PRA more than adequate. Others think that the current system is based primarily on goodwill and that this sentiment is not a strong enough premise to ensure ongoing cooperation with the lead response agency.

Regardless of mixed views, it can be concluded that the absence of clear supporting legislation presents a number of difficulties for the major emergency planning process. Firstly, the research indicates that the PRA's obligations to the 2006 Framework are subordinate to their existing statutory obligations, such as their responsibilities regarding the Seveso Directive (Article 11 (111)) (Seveso Directive 2008) described in Chapter 10, and also to Paragraph 15(1) of the Fire Services Act (1981). While the fulfilment of obligations under all three roles (Seveso, fire and major emergency) can be considered interchangeable and somewhat complementary, there remain some concerns regarding the status and priority of the 2006 Framework. Secondly, while there exists a significant amount of goodwill currently amongst and between PRA and individual government departments, there is no guarantee that this will continue indefinitely. The 2006 Framework is merely a guidance document, which is couched in non-prescriptive language, using terms such as 'PRA should...' rather than 'PRA must...'. Consequently, if a government department or PRA is occupied with some other important issue or project, its obligation to major emergency preparedness and planning may be more difficult to enforce. Thirdly, the research indicates that the roles and responsibilities of groups such as the IDWG and the NWG tend to be blurred. Clearly defined roles and responsibilities grounded in legislation, for each PRA, government department and supporting group, ensures that no confusion or duplication of effort exists. Despite the various views of the Irish interviewees, it can be concluded that there are distinct advantages to Ireland following the practice of leading countries in this regard and placing all aspects of major emergency management on a statutory basis.

\subsubsection{Coordination and oversight role}

The results of this study demonstrate that the coordination and oversight role for major emergency planning and management in Ireland is vested in the Office of Emergency Planning (OEP). The majority of interviewees indicate that they are happy with the OEP, as a sub-section of the Department of Defence, discharging this 
role. However, some confusion exists as to the precise roles and responsibilities of the various official working groups, including the OEP. Indicative of this was the view of one of the participants to the study who believes that the Department of the Environment, Heritage and Local Government (DEHLG) has a de jure oversight mandate from government.

This study also provides evidence that a number of Irish interviewees believe that the oversight role for major emergency management should be closer to the centre of power in government. This is in line with the practices of many of the international countries examined as part of this research. These interviewees believe that the oversight function should remain with the Office of Emergency Planning, but under the Department of Taoiseach rather than Defence. However, notwithstanding these findings, it can be concluded that the current arrangement, with the oversight role vested in the Department of Defence through the OEP, is adequate. This conclusion is predicated on an important finding from the study that the Department of Taoiseach will intervene in a major emergency situation should any resistance or difficulty be encountered. Furthermore, any significant structural changes at this stage of the major emergency implementation programme, may cause confusion or hinder progress.

The research also reveals that there are mixed opinions amongst the participants to the study as regards whether the OEP should be given stronger oversight powers. The OEP itself is emphatic that its original mandate allows it merely to oversee emergency planning, not take it over. This sentiment is supported by some of the other interviewees, including the Minister for Defence. However, an ardent school of thought exists that the OEP should be given a more robust mandate and autonomy in order to compel departments and PRA to plan, train, exercise and be assessed and to provide 'hard-hitting' reports where necessary. It can be concluded that this would be in line with best-international-practice. However, it can also be concluded that this may prove difficult to implement due to the current structure and staffing levels of the OEP. Additionally, without underpinning major emergency legislation, there is no statutory obligation on agencies or departments to comply with the requirements of the 2006 Framework. 
While there are mixed views in relation to bolstering the role and oversight powers of the OEP, the research results indicate that there is near unanimous support for putting in place a dedicated centralised major emergency budget, to be administered and disbursed by the OEP. It can be concluded that this initiative has particular merit and follows the trend of leading countries in the field of major emergency management. It is suggested here that this initiative might most profitably be considered as part of a much wider examination and comprehensive review of the role and powers of the OEP in the first instance.

\subsection{Major emergency communication - International context}

\subsubsection{Improvements in the theory and practice}

The data from the research confirm a fundamental hypothesis, namely that the communication function is central to the success of the overall major emergency management process. This is clearly reflected in the increased prominence of the communication management function in the major emergency literature from each of the countries examined, together with the high scores provided by the international interviewees when asked to rate the importance of the major emergency communication function. The many communication initiatives undertaken by the three agencies provide further evidence of the increased prominence of communication within major emergency management. For example, each of the three countries examined has established a dedicated communication section within its respective emergency management agency (FEMA, SEMA and CCS) in order to progress this important field of endeavour. It is apparent that the three agencies populate their respective communication sections with well-qualified communication practitioners who have the ability to provide strategic communication support and guidance to the overall preparedness and response effort.

Significant advances have taken place in the development of major emergency communication theory over the past number of decades. As the research demonstrates, a number of leading countries have made great strides in proactively producing guidance and information material of high quality for both their indigenous 
communication practitioners and their international counterparts. This includes research data, literature for communication practitioners, businesses and the voluntary sector, and the dissemination of useful information for members of the public, including booklets, DVDs and websites. Some countries, such as Australia and the US, have recognised the need to produce specialised information for use by marginalised groups such as children and people with disabilities. Innovative developments such as these are an indication of how communication management has become progressive. Best-practice, therefore, highlights the importance of building an indigenous repository of communication knowledge, through research, evaluation, case study and experiential learning that is available to all major emergency communication practitioners.

In relation to the practice of major emergency communication, the research highlights that leading countries view the senior major emergency communication manager's role as more than that of a mere implementer of other people's decisions. As described in Chapter 4, the major emergency communication function is utilised to optimum effect. The international agencies examined ensure that the senior communication practitioner has the ability and status to be part of the major emergency decision-making function at the highest level, or at least has direct access to senior decision-makers. The data also makes clear that the overseas agencies examined believe that their overall senior decision-makers have embraced the strategic communication principles and have accepted that the communication practitioner has an important role to play. Additionally, it can be concluded that leading countries no longer accept that major emergency communication teams are mere technicians but regard them as experts in media management, information handling, communication research and evaluation, and community liaison.

\subsubsection{Approach major emergency communication strategically}

It is evident from this research that leading countries are increasingly managing their major emergency communication function in a strategic manner rather than the more traditional tactical, short-term approach. Additionally, the communication literature reveals that major emergency communication guidance and support documents of leading countries are grounded in a strategic philosophy. In relation to major 
emergency communication strategy, leading countries tend to follow a similar series of steps beginning with the setting of clear, attainable and measurable communication goals. This is usually followed by a number of other important steps such as the undertaking of communication research, engaging stakeholders in dialogue, planning and evaluation. Leading communication practitioners emphasise that the 'management-by-objectives' process obliges the response agency to have a greater focus on achieving results or impacts than on merely implementing processes. In general terms, major emergency response agencies are public service organisations. Consequently, achieving major emergency communication targets rather than merely implementing communication processes may require an organisational change of culture and mindset. Despite this, the research clearly highlights that achieving results from the implementation of communication programmes makes the senior communication practitioner more accountable and also provides evidence of value for money for the government and the taxpayer. It can also be concluded from the research that countries engaging in best-practice ensure that they align and integrate their communication goals with those of the overall emergency plan, and that all communication goals are clearly understood by all response agencies. As communication management for major emergency situations is a discipline that has received detailed attention only in recent decades, the efficacy of approaching the field of endeavour strategically remains to be categorically proven through a process of research and evaluation.

Leading countries indicate that the major emergency communication planning process is of fundamental importance. There is consensus amongst the international interviewees that major emergency communication planning allows practitioners to anticipate problems and to respond effectively, and helps to reduce the number of unknowns. A close examination of the literature reveals that countries engaging in best-practice employ a set of prescribed major emergency communication planning steps. These steps follow closely those proposed in the suggested framework described earlier in Chapters 6, 7, and 8, which are designed to guide the major emergency communication practitioner throughout all phases. They include the planning and preparation of the communication function during the pre-crisis stage; implementing the communication response, including important tasks such as dealing with the media, issuing public warnings and delivering instructing information during 
the crisis stage; and communicating effectively and compassionately with victims, families and communities in the post-crisis stage. Significantly, countries employing best-practice plan their major emergency communication for the short-, medium-, and long-term. Additionally, the data indicate that leading countries build in flexibility in their plans by employing some form of anticipatory planning measures such as scenario planning, already described in Chapter 5. All three agencies place particular emphasis on the necessity to consider major emergency communication plans as living documents that require constant revision and updating.

\subsubsection{Approach communication scientifically}

In addition to approaching major emergency communication strategically, the research data highlight that countries engaging in best-practice approach the field of endeavour from a scientific perspective. A critical element of this scientific approach, as highlighted by theorists such as Reynolds, Coombs and Darrell (discussed in Section 5.3.3), and supported by the international interviewees, is that their communication practitioners possess some form of communication-related education. Without adequate education of this kind, the research suggests that it is difficult for the practitioner to examine new communication approaches and models, engage in communication research or evaluation, and have sufficient credibility to be given the opportunity to become involved in decision-making at the highest levels. It can be concluded from these results that communication teams are better able to support the overall major emergency effort with the knowledge that communication courses can provide. Communication practitioners also benefit from major emergency experience, either through actual situations or simulated exercises. According to the interviewees, this is considered equally as important as possessing communication-related education. Best-practice indicates that communication education and experience are complementary and that practitioners should possess a sufficiency of both in order to be effective.

All three overseas countries that were examined consider ongoing and iterative communication research to be a very important scientific element of the strategic communication process. The research shows that leading countries engage in some form of ongoing research, using both in-house and external researchers. It can be 
concluded that leading countries allocate requisite, well-trained resources to their lead response agencies in order to carry out the research function. According to all three respondents, the majority of this communication research is conducted in the preparedness and planning stage. However, significantly, all three countries also attach considerable importance to engaging in communication research in the period following the actualisation of a major emergency. The data indicate that they have adopted a broad focus for this research, including the state of public opinion; measurement of public perceptions, attitudes, needs and likely reactions in the face of a major emergency; and levels of trust in the response agencies. According to the interviewees, the majority of this research is conducted through focus groups, attitude surveys and ongoing consultation with specific interest groups.

It can also be concluded that leading countries consider the scientific process of evaluating major emergency communication to be very important. From the study, it is evident that countries engaging in best-international-practice evaluate their communication programmes throughout all stages of the major emergency life cycle and revise these programmes accordingly. Additionally, it is clear that leading countries evaluate both the process of planning major emergency communication programmes and their implementation, and also the impact that these programmes have on the recipients. All interviewees indicate that the communication evaluation process can only be effective if major emergency communication goals and objectives are stated at the outset. The research reveals that leading countries use both internal and external practitioners to conduct the evaluation activity. It can be concluded that these countries provide adequate, well-trained resources to their lead response agencies in order to carry out this important task.

\subsubsection{Integrated Communication}

Another significant finding that emerges from the research is that strategic major emergency communication is considered by leading countries to be an allencompassing, multidimensional and integrated discipline involving many diverse elements such as media management, public information, the raising of public awareness, internal communication, public affairs, community relations, and survivor and victim relations. This is a notable progression from the 1980s and early 1990s 
when communication management was considered merely in terms of media handling and public information. Pushing out the boundaries of further integration, as this research has indicated, FEMA has recently created a single, integrated model that allows better coordination and flows of all categories of major emergency data and that uniquely joins up the communication and information management functions. While the interviewees from both SEMA and CCS see considerable merit in FEMA's initiative, their organisations have not adopted this approach at time of writing. However, as this integrated model is available on the FEMA website and is undoubtedly discussed at international major emergency conferences, it would not be surprising to see other leading countries following FEMA's lead on this matter.

\subsubsection{Model of communication}

Leading countries state in their major emergency literature that they have adopted open and transparent models of major emergency communication when dealing with their stakeholders and publics. This is confirmed by the information provided by the international interviewees and demonstrated by a willingness to produce critical reports following major emergency situations. All of the international interviewees attach considerable importance to ensuring honesty, openness and transparency when providing important major emergency information to members of the public, regardless of the anxiety it might cause. They also demonstrate a willingness to become involved in meaningful dialogue with key stakeholders and members of the public. This commitment to openness, transparency and dialogue relates to all stages of the major emergency life cycle. As is the case with integrated communication, it can be concluded that this is a significant positive change that has occurred over the past two decades. However, while there is clear evidence of greater openness and transparency, it is difficult to measure precisely how much this has improved in each country and whether full openness and transparency is possible or prudent.

It is evident that the acquisition of formal communication-related education is helping practitioners to recognise the many advantages of open models of major emergency communication. Consequently, it can be concluded that countries engaging in bestinternational-practice are increasingly adopting a two-way symmetrical or mixed- 
motive model, when managing communication with their publics and stakeholders throughout all stages of a major emergency.

\subsubsection{Meaningfully engage the public}

Leading countries are now treating their citizens as partners when it comes to making important decisions that may affect them. A close examination of the major emergency communication literature reveals that this process is becoming increasingly important to the success of the overall preparedness and response effort. It is evident from the data that leading countries perceive many advantages in the public engagement process, including increased public support in relation to decisions taken, tapping into a greater supply of non-expert knowledge, achieving greater transparency in major emergency decision-making processes and maintaining greater accountability.

This study also reveals that a school of thought exists that perceives no discernible merit in engaging the public in major emergency decision-making. To this school, such a process causes delays, prevents decisions being reached, provides no accountability for the 'public' element of the decision-making process, and in many cases gives more weight to the opinion of the non-expert over that of the expert. Sceptics also highlight the difficulties in generating sufficient interest amongst a potentially apathetic public in order to allow a sufficiently broad base of interests and opinions to be represented. However, notwithstanding any disadvantages, the interviews with each of the three leading agencies provide strong support for the public engagement process. In this context, it should be noted, all of the interviewees highlight the importance of communication practitioners having the necessary expertise and knowledge to engage meaningfully with stakeholders and publics. They also emphasise that clear protocols and procedures must exist in order to deal with all of the shortcomings, such as having in place mechanisms for choosing the participants in the process and in the actions to be taken in the event that no agreement can be reached. Consequently, it can be concluded that countries that are considered leaders in the field of major emergency communication attempt meaningfully to engage their stakeholders and publics in decisions that may affect them, throughout all stages of a major emergency life cycle. 


\subsubsection{Building trust and credibility}

Leading countries increasingly recognise the importance of good communication in order to build trust and credibility amongst their publics, key stakeholders and the media, when planning for and responding to major emergency situations. It is evident that these countries spend considerable time and effort attempting to build and maintain levels of trust and credibility throughout all stages of a major emergency. Additionally, the data identifies a number of key elements that are considered essential to build and maintain trust and credibility in response agencies, including openness, transparency, truthfulness, providing a speedy communication response and keeping promises. It is clear that building and maintaining a high level of trust and credibility is a demanding process. Firstly, it is difficult to engage an apathetic public in matters related to major emergencies unless a disaster situation in imminent. This makes it difficult to promote the good preparedness efforts of response agencies during the pre-crisis stage. Secondly, because of the multitude of response agencies likely to be involved in managing a major emergency, the actions or words of a single member of any of these agencies can destroy the trust and credibility of the entire preparedness and response effort. Finally, the media, always looking for new and fresh angles to their reportage of major emergency issues, sometimes focus on negative or sensational events, such as deficiencies in the preparedness and response effort. This can significantly impact on the perception of public trust and credibility of response agencies. Leading countries have recognised problems such as these and as a consequence have afforded considerable resources, effort and time to the establishment of communication sections to help deal with such issues. It can be concluded from the study that a high level of public trust and credibility in the response organisation is extremely important for the overall success of the major emergency effort.

\subsubsection{Integrated communication training and exercises}

Leading countries are committed to integrating the communication function as an integral element of major emergency training and exercising. This is demonstrated by the involvement of communication practitioners in large-scale multi-agency major 
emergency exercises such as 'Exercise Topoff II' in the United States and 'Operation Hermes' in the United Kingdom. A close examination of the data highlights that integrating the communication function within all types of major emergency training and exercising provides many benefits. Benefits include allowing potential responders to understand the roles and responsibilities of communication practitioners and to recognise the value that the communication function adds, and also to develop familiarity with communication terminology, protocols and procedures. It can also be concluded that countries engaging in best-practice participate in integrated communication training and exercises on a regular basis and produce comprehensive, publicly available, reports in order to improve overall performance.

\subsection{Major emergency communication management - Irish context}

\subsubsection{The importance of major emergency communication}

This study clearly demonstrates that all Irish departments and response agencies examined consider the communication function a critical factor in the success or failure of the overall major emergency effort. However, unlike in the case of the leading international agencies examined, this recognition of the importance of the communication function is not evidenced to any great extent within the major emergency literature produced by departments and agencies to date, or by the implementation of communication-related initiatives. This is with the exception of a small number of initiatives already mentioned in Chapter 10, including the development of the user-friendly and informative OEP website, the major emergency handbook, and the communication sections of major emergency plans developed for responding to influenza pandemic and 'foot-and-mouth'. It can be concluded from the study that major emergency planners recognise the importance of major emergency communication. However, it can also be concluded that major emergency planners affording a higher priority to certain major emergency functions (such as operations) inadvertently hinder the development of all elements of a fully integrated major emergency communication function. While the communication function should not in any way hinder the operations effort, it should be developed and implemented in order to allow it to support and complement operations at all stages. However, while 
a somewhat similar model of major emergency communication is evolving internationally, the interviews conducted for this study indicate that any communication strategy that is developed and implemented in Ireland needs to take cognisance of the nuances of Irish culture. Practices and policies that are likely to work in the USA or Sweden may not necessarily be effective in the Irish context.

\subsubsection{Appreciation of major emergency communication}

Senior major emergency planners in leading countries refer to the practical benefits of strategic major emergency communication. However, with a small number of exceptions, it appears that within the relevant Irish departments and PRA there exists a limited appreciation of certain strategic communication principles and of the benefits of the strategic major emergency communication function. While the research data highlights that the majority of interviewees are quite familiar with many of the concepts and principles of strategic operations management, without a comprehensive appreciation of the key concepts of strategic major emergency communication, or how all of the diverse elements of an integrated communication strategy can benefit the overall major emergency management effort, senior planners are never likely to embrace fully the process. Furthermore, this is likely to impede the development of a fully integrated communication function within individual departments and agencies. This understanding can only be achieved through a process of education, demonstration and comparison with best-international -practice.

\subsubsection{Integrated communication}

As mentioned earlier in this chapter, countries engaging in best-international-practice consider major emergency communication to be a comprehensive, all-encompassing and integrated function involving many diverse elements such as media management, public awareness and information, public affairs, community relations, and survivor and victim relations. A number of the major emergency planners in Ireland consider major emergency communication merely in terms of media handling or the one-way asymmetrical dissemination of messages to the public. Interviewees who have an understanding of the concepts of integrated communication indicate that the 2006 Framework does not adequately provide for the integration of all of the diverse 
communication elements. Additionally, it can be concluded that the 2006 Framework treats information and communication management as distinct and independent functions. Many Irish major emergency planners see merit in a closer integration of these two important functions in order to more effectively manage major emergency situations, in line with best- international-practice.

Countries engaging in best-international-practice produce their major emergency communication guidance documents from one central source. For example, the Swedish Emergency Management Agency produces all of the communication-related guidance documents for that country. As mentioned earlier in this dissertation, major emergency planners in Ireland have undertaken a small number of useful communication programmes designed to guide and inform communication practitioners from departments and agencies, and the general public. These programmes are being developed by individual organisations somewhat in isolation. For example the OEP produced the major emergency handbook with the assistance of an external public relations firm, while the Garda Press Office are in the process of producing the communication guidance document (which relates strictly to media handling during a major emergency and would be better titled 'a media handling guidance document'). An approach such as this is not in line with best-internationalpractice, and is likely to lead to a lack of cohesiveness and consistency in the development of all guidance documents related to the major emergency communication function.

\subsubsection{Long-term communication planning}

Countries engaging in best-international-practice prepare strategies and plans for major emergency communication in the short-, medium-, and long-term. This involves putting in place multidimensional communication strategies and plans that help to guide the long-term direction of the communication effort. It also entails building up the communication knowledge base of individual departments and agencies, incrementally strengthening public information and awareness, and helping to promote community resilience over a prolonged period. A central focus of the longer-term communication strategy should be to modify attitudes towards individual and societal protection and resilience, by fostering a sense of interest amongst the 
public in major emergency issues. As discussed in Chapter 2, long-term communication strategising and planning has resulted in the implementation of successful community resilience programmes to help protect against hurricanes in less developed countries such as Haiti and Cuba. It can be concluded from the research data that Ireland's 2006 Framework takes very little cognisance of the longer-term aspects of major emergency communication preparedness. Indeed, it is very difficult to ascertain any focus beyond the two-year implementation stage of the Framework. It cannot be determined at this time whether this omission will be recognised and rectified within the forthcoming communication guidance document, which is currently under development. The research reveals that there are mixed views amongst senior major emergency planners in relation to undertaking long-term communication strategies, plans and initiatives. Some believe that major emergency management in Ireland, including the communication function, has been neglected and that significant 'catch up' is required. Consequently, these participants contend that the focus should be placed solely on short-term communication initiatives. However, others believe that, without a longer-term focus, the communication function does not provide an adequate strategic focus, disregards best-internationalpractice, and is not in the best interests of building up community major emergency knowledge, information, awareness and resilience.

\subsubsection{Departmental and agency communication practitioners}

Individual departments and agencies in Ireland do not recruit their communication practitioners based on their previous communication-related knowledge or experience. The vast majority of communication practitioners are recruited from other non-communication-related positions within their respective organisations.

Additionally, very few of these practitioners have undertaken any formal communication education or gained prior major emergency communication experience prior to taking up their role as a departmental or response agency communication practitioner (normally the role of a press officer). Each of the Irish departments and PRAs interviewed emphasises that its respective senior communication practitioner is a well-experienced press officer who, in many cases, has undertaken courses of training such as media skills courses. These communication practitioners also participate in any major emergency training or 
exercises that take place within individual departments and agencies. As the process for developing and implementing major emergency plans for individual departments and agencies remains incomplete, it is very difficult to ascertain from this research whether the process of recruiting communication practitioners to departments and agencies in Ireland is effective. However, when asked whether it would be better to target future communication practitioners within departments and agencies a number of years in advance, and to provide them with appropriate training and experience prior to taking up their posts, the majority of interviewees agreed. Nevertheless, some interviewees identified certain difficulties with this process, such as limiting the promotion opportunities of individuals who are obliged to follow such a route.

Additionally, as the implementation phase of the 2006 Framework initiative is not sufficiently progressed, it is too early to conclude that the skill set of communication practitioners within individual departments and agencies is sufficient to strategise, plan and implement the many diverse elements of the integrated strategic major emergency communication function as described in Chapter 5.

\subsubsection{Centralised guidance structure}

As discussed earlier in Chapter 9, leading countries in the field of major emergency management have established dedicated communication sections within their emergency management agencies. Practitioners within these sections have both the requisite communication knowledge and experience needed to provide useful strategic and tactical communication guidance and advice to individual response agencies. A notable conclusion that is drawn from the research data is that this expert major emergency communication guidance medium is not available to departments and agencies in Ireland. It is clear that no dedicated section or agency has been tasked with coordinating the many disparate elements of the strategic major emergency communication function, to provide guidance and advice to all response agencies, to develop a central repository of communication knowledge for use by all departments and agencies, and to advance the public information and awareness agenda.

Confusion exists amongst departments and agencies as to which organisation is responsible for providing this strategic communication guidance. This has not been clearly established within the 2006 Framework, thereby providing another example of 
the need to assign clear roles and responsibilities on a statutory basis. While the majority of interviewees identify either the Government Information Services (GIS) or the OEP as the two obvious sources for providing strategic communication guidance, it is clear from the data that neither GIS nor OEP believes the communication guidance function is in fact theirs. Furthermore, the current establishments and skill-sets of both the GIS and OEP are not sufficiently developed to allow them, at present, to become the central major emergency communication guidance organisation and repository of information. A role such as that requires considerable resources and expertise in order to be effective.

\subsubsection{Greater transparency and dialogue with members of the public}

Major emergency departments and agencies in Ireland have achieved greatly enhanced levels of openness and transparency in their communication with members of the public over the past five years. This is in line with best-international-practice. The research data confirm that, up until quite recently, a considerable amount of secrecy surrounded major emergency related issues in this country. Reduced secrecy is evidenced by a number of recent initiatives such as the posting of many official documents on the OEP website, inviting the media to visit the newly established National Emergency Coordination Centre (NECC) and providing a commitment that members of the media are to become participants in major emergency exercises. Nevertheless, important documents such as the OEP Annual Report, which provides details on the performance of each individual department in respect of major emergency preparedness and planning, remain classified as 'Secret' at time of writing and thus unavailable for public scrutiny.

From an analysis of international major emergency literature, it is evident that leading countries have demonstrated a commitment to increasingly involving members of the public in the decision-making process for major emergencies. The research data highlight that senior major emergency planners in Ireland provide equivocal support for engaging the public in decisions relating to major emergency situations. While many of the interviewees highlight distinct advantages to the public engagement process, such as acquiring useful information and achieving better compliance, the data reveal that there is currently no great appetite for implementing the process 
within departments and response agencies. The reasons for this inertia include a lack of requisite skill to carry out the public engagement function and a hesitancy on the part of senior decision-makers to consider new approaches.

It has been found that in order for major emergency management to be successful, members of the public need to have high levels of trust and credibility in response departments and agencies. Interviewees highlight the elements necessary for achieving trust and credibility - including demonstrating sufficient expertise, experience, openness and transparency. Public trust and credibility in the past have been significantly damaged by isolated events such as Joe Jacobs' radio interview and the mishandling of Iodine tablets, such as those discussed in Chapter 11. The interview results also indicate that the public's current negative perception of the Health Services Executive (HSE), based on experiences and criticisms of the health service, may pose a significant problem for major emergency planners in the event of a serious influenza pandemic occurring.

\subsection{Key recommendations for Ireland}

Four key recommendations are made in the light of these conclusions.

1. Taking cognisance of the model of major emergency communication currently preferred by many leading countries, major emergency planners in Ireland should conduct research in order to determine whether a similar model, involving a two-way symmetrical or mixed-model of communication management, would prove beneficial in supporting the major emergency manager in the Irish context. As demonstrated in this dissertation, such a relational-based approach has a broad base of support across a range of communication sub-specialities. This includes support from contemporary communication theorists, including Grunig (p59), Murphy (p60), Cutlip, Center and Broom (p55) and Susskind and Field (p55); from major emergency communication theorists such as Nohrstedt (p88), Reynolds and Nordlund (p89); and from major emergency response organisations including FEMA, SEMA and CCS (Section 9.4.3). Adopting such a model of communication 
would reflect the international shift in how major emergency management is approached, whereby contemporary response agencies tend to plan with communities rather than for communities, as discussed in Figure 2.1 (p28). However, as described in Section 5.3.1, this shift towards preferring a dialogical and relational-based model to major emergency communication is a somewhat recent phenomonen. In addition to conducting a comprehensive cost-benefit analysis, this model needs to be rigorously tested, both in exercises and also in live situations, before it can be deemed to be truly effective in the Irish context.

2. In order to overcome the many disadvantages of engaging in tactical communication management, described in Section 4.3.1 (p76), response agencies in Ireland should study, research and test all elements of the six strategic major emergency communication principles, proposed in Section 5.3 (p93), in order to determine whether they can be applied and would be beneficial within the Irish context. In particular, Irish major emergency communication practitioners need to consider a greater evidenced-base approach to communication information, involving research and evaluation, as outlined earlier by emminent theorists such as Broom, Dozier, Grunig and Cutlip (p83 and p84). By adopting such strategic communication principles, Ireland's major emergency communication approach would be in line with that of leading international organisations such as FEMA, SEMA, CCS, EMA, and Public Safety Canada.

3. As part of this strategic approach, Irish response agencies need to consider the management of major emergency communication as more than simply media management and the provision of public information. In this regard, the Irish major emergency communication practitioner needs to regard the strategic communication function as an all-encompassing and integrated discipline involving subsidiary important functions such as internal communication, community relations, survivor and victim relations, and public affairs (as discussed in Section 5.3.5 (p111)). At a minimum, strong links and coordination needs to be established between the communication practitioner and these subsidiary functions. 
4. Finally, in choosing the preferred major emergency communication model, or in adopting any or all of the strategic communication principles presented in the conceptual framework, the Irish major emergency communication practitioner needs to consider the role of national and local cultural factors. As indicated by theorists such as Mars and Nicod (p48), Thompson, Ellis and Wildavsky (p48), Marra (p100), and Frosdick (p100), communication strategies that work well for one group of individuals may not necessarily work for others. Regardless of what is considered best-practice, any major emergency communication strategy that is developed and implemented for Ireland will have to be tailored to take cognisance of the specificities of Irish culture. 


\section{BIBLIOGRAPHY}




\section{Bibliography}

\section{Books}

Adams, John. 1995. Risk. London: Routledge.

Alexander, David. 2002. Principles of emergency planning and management. Hertfordshire: Terra.

Arquilla, John and Ronfeldt, David J. 1997. In Athena's camp: preparing for conflict in the information age. Santa Monica, CA: Rand.

Asch, David and Bowman, Cliff. 1989. Readings in strategic management. London: Open University.

Auf der Heide, Erik. 1989. Disaster response: principles of preparation and coordination. Amsterdam: Elsevier.

Augustine, Norman R. 2000. Harvard business review on crisis management. Boston: Harvard Business School Press.

Austin, Erica W. and Pinkleton, Bruce E. 2000. Strategic public relations management: planning and managing effective communication programmes. NJ: Lawrence Erlbaum Associates.

Beck, Ulrich. 1992. Risk society. London: Sage.

Benthall, Jonathan. 1993. Disaster relief and the media. London: IB Tauris.

Berge, Dieudonnee T. 1990. The first 24 hours. Oxford: Blackwell Publishing.

Berger, Charles R. and Chaffee, Steven H. 1987. Handbook of communication science. London: Sage.

Berlo, David K. 1960. The process of communication. New York: Holt, Rinehart and Winston.

Bernstein, Alan B. 1987. The emergency public relations manual, $3^{\text {rd }}$ edition. Highland Park, NJ: PASE Inc. 
Bernstein, Richard. 2002. Out of the blue. New York: Henry Holt and Company.

Bowman, Cliff. 1990. The essence of strategic management. London: Prentice Hall.

Broom, Glen M. and Dozier, David M. 1990. Using research in public relations. London: Prentice Hall.

Bulmer, Martin (ed.). 2004. Questionnaires: volume 1. London: Sage.

Burton, Ian and Kates, Robert W. and White, Gilbert F. 1993. The environment as hazard. New York: Guilford Press.

Canter, David (ed.). 1980. Fires and human behaviour. New York: John Wiley and Sons.

Casmir, Fred L. 1991. Communication in development. NJ: Ablex Publishing.

Civil Defence (Ireland). 1961. Bás beatha. Dublin: Stationery Office.

Clemen, Robert and Reilly, Terence. 2001. Making hard decisions with decision tools. CA: Duxbury.

Cohn, Robin. 2000. The PR crisis bible. New York: St. Martin's Press.

Comfort, Louise K. (ed.). 1988. Managing disasters: strategies and policy perspectives. Durham: Duke University Press.

Cooley, Charles H. 1909. Social organization: a study of the large mind. New Brunswick, NJ: Transaction Books.

Coombs, Timothy W. 1999. Ongoing crisis communication: planning, managing, and responding. London: Sage.

Coppola, Damon P. 2007. Introduction to international disaster management, $7^{\text {th }}$ edition. Oxford: Elsevier.

Covello, Vincent T. and Allen, Frederick. 1988. The EPA's seven cardinal rules of risk and crisis communication, OPA-87-020. Washington, DC: Environmental Protection Agency. 
Creswell, John W. and Plano-Clark, Vicki L. 2007. Designing and conducting mixed methods research. Thousand Oaks, CA: Sage.

Culbertson, Hugh M. and Chen, Ni. 1996. International public relations: a comparative analysis. NJ: Lawrence Erlbaum Associates.

Cuny, Frederic C. 1983. Disasters and development. Oxford: Oxford University Press.

Cushman, Donald P. and Sanderson-King, Sarah (eds). 2001. Excellence in communicating organizational strategy. Albany: State University of New York Press.

Cutlip, Scott M. and Center, Alan H. and Broom, Glenn M. 2000. Effective public relations, $8^{\text {th }}$ edition. Englewood Cliffs, NJ: Prentice Hall.

Czitrom, Daniel J. 1982. Media and the American mind: from Morse to McLuhan. Chapel Hill, NC: University of North Carolina Press.

Darrell, Birgitta. 2003. Crisis communication handbook. Stockholm: Swedish Emergency Management Agency.

David, Fred R. 1993. Strategic management, $4^{\text {th }}$ edition. London: Macmillan Publishing.

Deacon, David and Pickering, Michael and Golding, Peter and Murdock, Graham. 1999. Researching communications. New York: Arnold.

Denzin, Norman. 1978. Sociological methods: a sourcebook, $2^{\text {nd }}$ edition. NY: McGraw Hill.

Dervin, Brenda and Voigt, Melvin J. 1989. Progress in communication sciences. Norwood, NJ: Ablex.

DeVito, Joseph. 1997. Human communication: the basic course. New York: Longman.

Dewey, John. 1927. The Public and its Problems. New York: Holt. 
DeWit, Bob and Meyer, Ron. 2004. Strategy: process, content, context, $3^{\text {rd }}$ edition. London: Thomson.

Dickson, David. 1997. Artic Ireland. Belfast: White Row Press.

Dora, Carlos. 2006. Health hazards and public debate: lessons for risk communication from the BSE/CJD saga. Copenhagen: WHO.

Dowie, Jack and Lefrere, Paul (eds). 1980. Risk and chance: selected readings. Buckingham: Open University Press.

Dozier, David M. and Grunig, James E. and Grunig Larissa A. 1995. Managers guide to excellence in public relations and communication management. $\mathrm{NJ}$ : Lawrence Erlbaum Associates.

Drabek, Thomas and Hoetmer, Gerard (eds). 1991. Emergency management: principles and practice for local government. Washington, DC: International City Management Association.

Dranove, David and Marciano, Sonia. 2005. Kellogg on strategy. NJ: John Wiley and Sons.

Eagly, Alice H. and Chaiken, Shelley. 1993. The psychology of attitudes. Fort Worth, Texas: Harcourt Brace Jovanovich.

Ellul, Jacques. 1965. Propaganda: the formation of men's attitudes. New York: Alfred A Knopf, Inc.

Fearn-Banks, Kathleen. 1996. Crisis communication: a casebook approach. NJ: Lawrence Erlbaum Associates.

Fernandez, Louie and Merzer, Martin. 2003. Crisis communication handbook. Virginia: Janes Publications.

Fink, Steven. 1986. Crisis management: planning for the inevitable. New York: Amacom.

Finlay, Paul. 2000. Strategic management: an introduction to business and corporate strategy. London: Prentice Hall. 
Fischhoff, Baruch. 2004. Mental models of warning decisions: identifying and addressing information needs. IN: Wogalter, Michael S. (ed.). Handbook of warnings. NJ: Lawrence Erlbaum Associates.

Fiske, John. 2004. Introduction to communication studies, $2^{\text {nd }}$ edition. London: Routledge.

Friend, John and Hickling, Allen. 1997. Planning under pressure. Oxford: Butterworth-Heinemann.

Friere, Paulo. 1983. Pedagogy of the oppressed. New York: Continuum.

Gamble, Teri Kwal and Gamble, Michael. 2005. Communication works, $8^{\text {th }}$ edition. New York: McGraw Hill.

Gist, Richard and Lubin, Bernard. 1989. Psychosocial aspects of disaster. New York: John Wiley.

Gough, Janet and Hooper, George. 2003. Sharing the future: risk communication in practice. Canterbury: Centre for Advanced Engineering.

Grant, Robert M. 2008. Contemporary strategy analysis: concepts, techniques, applications, $6^{\text {th }}$ edition. Oxford: Blackwell Publishers.

Griffin, Em. 2003. A first look at communication theory, $5^{\text {th }}$ edition. New York: McGraw Hill.

Grunig, James E. (ed.). 1992. Excellence in public relations and communication management. NJ: Lawrence Erlbaum Associates.

Grunig, James E. and Hunt, Todd. 1984. Managing public relations. New York: Holt, Rinehart and Winston.

Grunig, Larissa A. and Grunig, James E. and Dozier, David M. 2002. Excellent public relations and effective organisations: a study of communication management in three countries. NJ: Lawrence Erlbaum Associates.

Handmer, John (ed.). 1990. Hazards and the communication of risk. Aldershot: Gower Technical. 
Hardt, Hanno. 1992. Critical communication studies: communication history and theory in America. London: Routledge.

Heath, Robert L. (ed.). 2001. Handbook of public relations. London: Sage.

Hewitt, Kevin. 1997. Regions of risk: a geographical introduction to disasters. Harlow: Addison-Wesley-Longman.

Higgins, James M. 1979. Organisational policy and strategic management: texts and cases. Hinsdale, Illinois: Dryden.

Hill, Charles W. and Jones, Gareth R. 2001. Strategic management: an integrated approach. Boston: Houghton Mifflin Company.

Hodgkinson, Peter E. and Stewart, Michael. 1991. Coping with catastrophe. London: Routledge.

Hofstede, Geert. 1980. Culture's consequences. Beverly Hills, CA: Sage.

Hood, Christopher and Jones, David. 1996. Accident and design: contemporary debates in risk management. London: UCL Press.

Howarth, George. 1999. Foreword. IN: Harrison, Shirley (ed.). Disasters and the media: managing crisis communication. London: Macmillan Press.

Ingleton, Jon. 1999. Natural disaster management. Leicester: Tudor Rose.

Irwin, Alan and Wynne, Brian (eds). 1996. Misunderstanding science?

Cambridge: Cambridge University Press.

Iwan, Wilfred. 1999. Reducing disaster losses through better information. Washington, DC: National Academy of Sciences.

Jensen, Klaus B. and Jankowski, Nicholas W. 1991. A handbook of qualitative methodologies. London: Routledge.

Jeynes, Jacqueline. 2002. Risk management: 10 principles. Oxford: ButterworthHeinemann. 
Johnson, Gerry. 1992. Managing strategic change: strategy culture and action. IN: Faulkner, D. and Johnson, Gerry (eds). The challenge of strategic management. London: Kogan.

Johnson, Gerry and Scholes, Kevan and Whittington, Richard. 2008. Exploring corporate strategy, $8^{\text {th }}$ edition. London: Prentice Hall.

Johnson, Gerry and Scholes, Kevan. 1988. Exploring corporate strategy, $2^{\text {nd }}$ edition. London: Prentice Hall.

Johnson-Cartee, Karen S. and Copeland, Gary A. 2003. Strategic political communication. Lanham: Rowman and Littlefield.

Kamer, Larry. 1996. When the crisis is orchestrated: corporate campaigns and their origins. IN: Barton, Laurence (ed.). New avenues in risk and crisis management. Las Vegas, Nevada: UNLV Small Business Development Center.

Kaye, Michael. 1994. Communication management. London: Prentice Hall.

Kenny, Colum. 2003. Fearing Sellafield. Dublin: Gill and Macmillan.

Kleiner, Art. 1996. The age of heretics. New York: Currency Doubleday.

Lasswell, Harold. 1941. Democracy through public opinion. USA: George Banta Publishing Company.

Lasswell, Harold. 1948. The structure and function of communication in society. IN: Bryson, Lyman (ed.). The communication of ideas. New York: Harper and Row.

Lazarsfeld, Paul F. and Field, Henry. 1946. The people look at radio. Chapel Hill: University of North Carolina Press.

Leiss, William and Powell, Douglas. 1997. Mad cows and mother's milk: the perils of poor risk communication. Montreal: McGill-Queens University.

Lerner, Daniel. 1977. Communication and development. IN: Lerner, Daniel and Nelson, Lyle (eds). Communication research: a half-century appraisal. Honolulu: East-West Center.

L'Etang, Jacquie and Pieczka, Magda (eds). 1996. Critical perspectives in public relations. London: International Thomson Business Press. 
Lincoln, Yvonna S. and Guba, Egon G. 1985. Naturalistic inquiry. Beverly Hills, CA: Sage Publications, Inc.

Lindell, Michael K. and Prater, Carla and Perry, Ronald W. 2007. Introduction to emergency management. New York: John Wiley.

Lippmann, Walter. 1922. Public opinion. New York: The Free Press.

Lippmann, Walter. 1925. The phantom public. New York: Harcourt, Brace and Company, Inc.

Magnusson, Magnus (ed.). 1987. The Readers Digest book of facts, $2^{\text {nd }}$ edition. London: Readers Digest Association.

Mars, Gerald and Nicod, Michael. 1983. The world of waiters. London: Allen and Unwin.

McQuail, Denis and Windahl, Sven. 1993. Communication models, $2^{\text {nd }}$ edition. London: Longman.

McQuail, Denis and Golding, Peter and de Bens, Els (eds). 2005. Communication theory and research: an EJC anthology. London: Sage.

Mileti, Dennis S. 1999. Disaster by design: a reassessment of natural hazards in the United States. Washington, DC: National Academies Press.

Millar, Dan P. and Smith, Larry L. 2002. Crisis management and communication. CA: International Association of Business Communicators (IABC).

Miller, Gerald R. and Steinberg, Mark. 1975. Between people: a new analysis of interpersonal communication. Chicago: Science Research Associates.

Mintzberg, Henry. 1994. The Rise and fall of strategic planning. New York: Prentice Hall.

Mintzberg, Henry and Quinn, James B. 1991. The strategy process: concepts, contexts, cases, $2^{\text {nd }}$ edition. London: Prentice Hall. 
Modh, Malin. 2003. Crisis journalism: a guidance for government agencies. Stockholm: SEMA.

Morse, Janice M. 1994. Emerging from the data: the cognitive processes of analysis in qualitative inquiry. IN: Morse, Janice M. (ed.). Critical issues in qualitative research methods. Thousand Oaks, CA: Sage.

Mortensen, David C. 1997. Miscommunication. London: Sage.

Mould, Richard F. 1988. Chernobyl: the real story. Oxford: Pergamon Press.

National Research Council. 1980. Disasters and the mass media. Washington, DC: National Academy of Sciences.

Newsom, Doug and Turk, Judy and Kruckeberg, Dean. 1996. This is PR: the realities of public relations, $6^{\text {th }}$ edition. Belmont, CA: Wadsworth.

Nordlund, Roland. 1994. A triangle drama: authorities, citizens and media in crisis. Stockholm: SPF.

Norman, Sarah and Stuart-Black Jim and Coles, Eve (eds). 2006. Health emergency planning: a handbook for practitioners. Norwich: TSO.

Ogrizek, Michel and Guillery, Jean-Michel. 1999. Communicating in crisis. New York: Aldine de Gruyter.

O’Riordan, Patrick A. 1992. Emergency planning in Ireland. Dublin: IPA.

O'Sullivan, Tim and Hartley, John and Saunders, Danny and Montgomery, Martin and Fiske, John. 1994. Key concepts in communication and cultural studies. London: Routledge.

Pauchant, Thierry C. and Mitroff, Ian I. 1992. Transforming the crisis-prone organisation: preventing individual, organisational, and environmental tragedies. San Fransisco: Jossey-Bass.

Pearson, Judy and Nelson, Paul and Titsworth, Scott and Harter, Lynn. 2006. Human communication, $2^{\text {nd }}$ edition. Boston: McGraw Hill. 
Price, Roseanne (ed.). 1989. Improving risk communication. Washington, DC: National Academies Press.

Price, Stuart. 1996. Communication studies. Harlow: Longman.

Putnam, Robert D. 2000. Bowling alone. New York: Simon and Schuster.

Quinn-Patton, Michael. 2002. Qualitative research and evaluation methods. London: Sage.

Reason, James T. 1990. Human error. Cambridge: Cambridge University Press.

Redmond, Mark V. 2000. Communication: theories and applications. Boston: Houghton Mifflin.

Renn, Ortwin. 2008. Risk governance: coping with uncertainty in a complex world. London: Earthscan.

Reynolds, Barbara. 2002. Crisis and emergency risk communication. Atlanta: Centers for Disease Control (CDC).

Reynolds, Barbara. 2003. Crisis emergency risk communication: by leaders for leaders. Atlanta: Centers for Disease Control (CDC).

Ringland, Gill. 1998. Scenario planning: managing for the future. West Sussex: John Wiley.

Robin, Ron Theodore. 2001. The making of the cold war enemy: culture and politics in the military-intellectual complex. Princeton, NJ: Princeton University Press.

Robbins, Stephen P. 1990. Organisation theory: structure, design and applications, $3^{\text {rd }}$ edition. Englewood Cliffs, NJ: Prentice-Hall.

Robson, Colin. 2002. Real World Research, $2^{\text {nd }}$ edition. Oxford: Blackwell.

Rogers, Everett M. 1994. A history of communication study. New York: Free Press.

Rokeach, Milton. 1968. Beliefs, attitudes and values: a theory of organisation and change. San Francisco: Jossey Bass. 
Salwen, Michael B. and Stacks, Don W. 1996. An integrated approach to communication theory and research. NJ: Lawrence Erlbaum and Associates.

Scammell, Margaret and Semetko, Holi A. 2000. Media, journalism and democracy. Sudbury, MA: Dartmouth Publishing.

Schramm, Wilbur. 1997. The beginnings of communication study in America: a personal memoir. Newbury Park, CA: Sage Publications.

Scott, Michael D. and Powers, William G. 1978. Interpersonal communication: a question of needs. Boston: Houghton Mifflin.

Seedhouse, David. 1997. Health promotion: philosophy, prejudice and practice. NJ: Wiley.

Seitel, Fraser P. 2004. The practice of public relations, $9^{\text {th }}$ edition. NJ: Pearson Education.

Servaes, Jan and Jacobson, Thomas L. and White Shirley A. 1996. Participatory communication for social change. London: Sage.

Severin, Werner J. and Tankard, James W. 1988. Communication theories: origins, methods, uses, $2^{\text {nd }}$ edition. New York: Longman.

Shank, Gary D. 2006. Qualitative research: a personal skills approach. New Jersey: Pearson.

Slovic, Paul. 2000. The perception of risk. London: Earthscan.

Slovic, Paul and McGregor, David. 1994. The social context of risk communication. New Jersey: Decision Research Publications.

Smelser, Neil J. and Mitchell, Faith. 2002. Terrorism: perspectives from the behavioural and social sciences. Washington, DC: National Academies Press.

Smith, Keith. 1992. Environmental hazards: assessing risk and reducing disaster. London: Routledge. 
Smith, Mark C. 1994. Social science in the crucible: the American debate over objectivity and purpose 1918-1941. Durham: Duke University Press.

Smith, Ronald D. 2005. Planning for public relations. NJ: Lawrence Erlbaum.

Steiner, George A. 1969. Top management planning. New York: Macmillan.

Stern, Paul and Fineberg, Harvey V. (eds). 1996. Understanding risk: informing decisions in a democratic society. Washington, DC: National Academies Press.

Stiff, James B. 1994. Persuasive communication. New York: The Guilford Press.

Sun Tzu. 1971 (reprinted). The art of war. New York: Oxford University Press.

Susskind, Lawrence and Field, Patrick. 1996. Dealing with an angry public: the mutual gains approach. New York: The Free Press.

Thayer, Henry S. 1968. Meaning and action: a critical history of pragmatism. New York: Bobs-Merrill.

Theodorson, George A. and Theodorson, Achilles G. 1969. A modern dictionary of sociology. New York: Cassell.

Thompson, Arthur and Strickland, A. (Lonnie). 1995. Crafting and implementing strategy, $6^{\text {th }}$ edition. Homewood, Illinois: Richard D. Irwin.

Thompson, Marilyn W. 2003. The killer strain: anthrax and a government exposed. New York: Harper Collins.

Thompson, Michael and Ellis, Richard and Wildavsky, Aaron 1990. Cultural theory. Boulder, Colorado: Westview.

Tierney, Kathleen and Lindell, Michael K. and Perry, Ronald W. 2001. Facing the unexpected: disaster preparedness and response in the United States. Washington, DC: Joseph Henry Press.

Toft, Brian and Reynolds, Simon. 2004. Learning from disasters: a management approach. Leicester: Perpetuity Press. 
Van der Heijden, Kees. 1996. Scenarios: the art of strategic conversation.

Chichester: John Wiley and Sons.

Van Ruler, Betteke and Verčič, Dejan (eds). 2004. Public relations and communication management in Europe. The Hague: Mouton de Gruyter.

Walter, Jonathan (ed.). 2005. World disasters report. Geneva: International Federation of Red Cross and Red Crescent Societies.

Watzlawick, Paul and Beavin-Bavelas, Janet and Jackson, Don. 1967. Pragmatics of human communication. New York: W.W. Norton.

White, Shirley A. 1994. Participatory communication: working for change and development. London: Sage.

Wilcox, Dennis L. and Ault, Philip H. and Agee, Warren K. 1997. Public relations: strategies and tactics. New York: Longman.

Wilcox, Dennis L. and Cameron, Glen T. 2005. Public relations: strategies and tactics, $8^{\text {th }}$ edition. New York: Longman.

Wildavsky, Aaron. 1988. Searching for safety. New Brunswick, NJ: Transaction Press.

Williams, Frederick. 1992. The new communications. Belmont, CA: Wadsworth.

Wolbarst, Anthony (ed.). 2001. Solutions to an environment in peril. Baltimore, Maryland: John Hopkins University Press. 


\section{Official published documents and reports}

Academy for Crisis Management, Emergency Planning and Civil Relief and the European Union (AKNZ and EU). 2001. Crisis communication: final report [Online]. Available from:

http://ec.europa.eu/environment/civil/act_prog_rep/crisis_communication_en.pdf [Accessed 8 January 2008].

Adams, John and Thompson, Michael. 2002. Taking account of societal concerns about risk: framing the problem. London: HSE Books.

Alesch, Dan J. and May, Peter and Olshansky, Robert and Petak, William and Tierney, Kathleen. 2004. Promoting Seismic Safety: guidance for Advocates. Joint publication of the Mid-America Earthquake Center, the Multidisciplinary Center for Earthquake Engineering Research, and the Pacific Earthquake Engineering Research.

Allbaugh, Joe. 2003. A nation prepared: Federal Emergency Management Agency strategic plan 2003-2008. Washington, DC: FEMA.

Anderson, Iain. 2002. Foot and mouth disease 2001: lessons to be learned inquiry report. London: TSO.

Australian Standard. 1999. Risk management: AS/NZS4360:1999. Strathfield, NSW: Standards Association of Australia.

Ball, David J. and Boehmer-Christiansen, Sonja. 2002. Understanding and responding to societal concerns. HSE report no. RR034, 2002 [Online]. Available from: http://www.hse.gov.uk/research/rrpdf/rr034.pdf [Accessed 7 January 2008].

Beckett, Margaret. 2000. Modernising government in action: realising the benefits of $Y 2 K$. London: TSO.

Besser, Howard. 2004. The next digital divide [Online]. Available from http://www.tcla.gseis.ucla.edu/divide/politics/besser.html [Accessed 13 March 2006].

Cabinet Office. 2004. Dealing with disaster, $3^{\text {rd }}$ edition. London: Civil Contingencies Secretariat. 
Cabinet Office. 2005(a). The lead government department and its role-guidance and best practice. London: Civil Contingencies Secretariat.

Cabinet Office. 2005(b). Central government arrangement for responding to an emergency: concept of operations. London: Civil Contingencies Secretariat.

Central Statistics Office (CSO). 2004. Statistical yearbook of Ireland 2004. Dublin: Stationery Office.

Central Statistics Office (CSO). 2006(a). Census 2006: principal demographic results. Dublin: Stationery Office.

Central Statistics Office (CSO). 2006(b). Census 2006 volume 11: disability, carers and voluntary activities. Dublin: Stationery Office.

Commission for Communications Regulation (Comreg). 2007. Comreg latest quarterly report show broadband take-up continues to grow strongly [Online]. Available from http://www.comreg.ie/_fileupload/publications/PR181207a.pdf [Accessed 9 February 2008].

Coogan, Suzanne. 2007. Press release. Dublin: Department of Defence.

Crisis and Risk Network (CRN). 2003. Workshop report. Oslo: Directorate for Civil Protection and Emergency Planning Norway.

Dáil Éireann. 2004. Parliamentary debates: vol 593: 1 [Online]. Available from http://debates.oireachtas.ie [Accessed 25 March 2008].

Department of Agriculture, Fisheries and Food (DAFF). 2005. Annual report. Dublin: Department of Agriculture, Fisheries and Food.

Department of Defence. 2004. Strategic emergency planning guidance. Dublin: Office of Emergency Planning.

Department of Defence. 2005(a). Emergency planning: public attitudes survey. Dublin: Millward Brown IMS [posted on e-tenders website 22 November 2006].

Department of Defence. 2005(b). Draft memorandum for the government: public information campaign on emergency planning. Dublin: Office of Emergency Planning. 
Department of the Environment. 1981. Planning for major emergencies.Dublin: Department of the Environment.

Department of the Environment. 1984. Emergency planning: framework for coordinated response to major emergency. Dublin: Department of the Environment.

Department of Public Enterprise. 2002. Review of national emergency plan for nuclear accidents: final report. Dublin: Environmental Resources Management.

Eksborg, Ann-Louise. 2004. Experiences and conclusions after two years [Online]. Available from http://www.krisberedskapsmyndigheten.se [Accessed 11 August 2007].

Emergency disasters data-base (EM-DAT). 2008. Glossary [Online]. Available from http://ww.em-dat.net/glossary.htm [Accessed 10 May 2008].

Emergency Management Australia (EMA). 2003. Mapping the way forward for large-scale urban disaster management in Australia. Canberra: Attorney-General's Department.

Federal Emergency Management Agency (FEMA). 2004. Are you ready: an in-depth guide to citizen preparedness. Washington, DC: FEMA.

Fire Services Act. 1981. Irish statute book [Online]. Available from http://www.irishstatutebook.ie/1981/en/act/pub/0030/index.html [Accessed 15 June 2008].

Garda Síochána (GS). 2003. Chemical, biological, radiological, or nuclear $(C B R N)$ : operational response. Dublin: Garda Síochána.

Gaynor, Sally and McAteer, Billy. 2003. Foot and mouth disease: operations manual. Dublin: Department of Agriculture, Fisheries and Food.

Goverment Task Force on Emergency Planning. 2006(a). A framework for major emergency management. Dublin: Inter-Departmental Committe on Major Emergencies. 
Goverment Task Force on Emergency Planning. 2006(b). A framework for major emergency management: appendices. Dublin: Inter-Departmental Committe on Major Emergencies.

Health Service Executive Ireland (HSE). 2007. National pandemic influenza plan. Dublin: Department of Health and Children.

Health and Safety Executive United Kingdom (HSE UK). 1999. Quantified risk assessment: its input to decision making. London: HMSO.

HM Government. 2004. Civil Contingencies Act. London: Crown Copyright.

HM Government. 2005(a). Emergency preparedness: on part 1 of the Civil Contingencies Act 2004. London: Crown Copyright.

HM Government. 2005(b). Emergency response and recovery. London: Crown Copyright.

Inter-Departmental Liaison Group on Risk Assessment (ILGRA). 1998. Risk communication: a guide to regulatory practice. London: ILGRA.

Lasker, Roz D. 2004. Redefining readiness: terrorism through the eyes of the public. New York: The New York Academy of Medicine.

London Resilience. 2005. Strategic emergency plan. London: London Resilience Team.

Management of Risk (MOR). 2002. Guidance for practitioners. London: Office of Government Commerce.

McQuaid, Jim. 1997. Risk communication: a guide to regulatory practice. London: Inter-Departmental Liaison Group on Risk.

Morge, Sara. 2000. Alarm, inform: public warning systems in four countries. Stockholm: National board of psychological defence (SPF).

National Science and Technology Council (NSTC). 2000. Effective disaster warnings. Report by the working group on natural disaster information systems. Washington, DC: Executive Office of the President of the United States. 
Nolan, John. 2002. Emergency planning: annual report to government. Press release. November 18th 2002. Dublin: Department of Defence.

Norman, Sarah (ed.). 2004. Proceedings: New Zealand recovery symposium. Wellington: Ministry of Civil Defence and Emergency Management.

Nuclear Energy Board. 1987. Chernobyl and its effects on Ireland. Dublin: Nuclear Energy Board.

Nuclear Safety Section. 1992. National emergency plan for nuclear accidents (NEPNA). Dublin: Department of Public Enterprise.

Nuclear Safety Section. 2004. National emergency plan for nuclear accidents (revised) (NEPNA). Dublin: Department of the Environment, Heritage and Local Government.

Office of Emergency Planning (OEP). 2008. Preparing for emergencies: an introduction. Dublin: Office of Emergency Planning.

Pandemic Influenza Expert Group (Ireland). 2007. Pandemic influenza preparedness for Ireland: advice of the pandemic influenza expert group. Dublin: Health Services Executive.

PR Coalition. 2003. Restoring trust in business: models for action. NJ: Fairleigh Dickinson University.

Ridge, Tom. 2004(a). National incident management system (NIMS). Washington, DC: Department of Homeland Security.

Ridge, Tom. 2004(b). National response plan. Washington, DC: Department of Homeland Security.

Ridge, Tom. 2004(c). Securing our homeland. Washington, DC: Department of Homeland Security.

Royal Academy of Engineers. 2002. The societal aspects of risk. London: Royal Academy of Engineering.

Royal Society. 1992. Risk: analysis, perception and management. London: Royal Society. 
Seveso Directive. 2008. Seveso directive: article 11 (111). European

Commission: joint research centre [Online]. Available from http://mahbsrv.jrc.it/ [Accessed 15 June 2008].

Swedish Defence Commission. 1999. A changing world-a reformed defence. Stockholm: Swedish Ministry of Defence.

Taylor, Lord. 1990. Final Report on the Hillsborough Stadium disaster. HMSO: London.

Townsend, Frances (Chairman). 2006. The federal response to Hurricane Katrina: lessons learned. Washington, DC: Department of Homeland Security.

Trompenaars, Fons. 1993. Riding the waves of culture: understanding cultural diversity in business. London: The Economist Books.

UK Emergency Planning Society (EPS). 1998. Responding to disaster: the human aspects. London: Emergency Planning Society.

United Nations Department of Humanitarian Affairs (UN DHA). 1992. Internationally agreed glossary of basic terms related to disaster management. Geneva: DHA.

United Nations Disaster Relief Organisation (UNDRO). 1987. Disaster prevention and mitigation: a compendium of current knowledge, public information aspects. New York: United Nations.

US Central Intelligence Agency (CIA). 2007. The world factbook. Washington, DC: CIA.

US National Response Team (NRT). 2000. Joint information center model: collaborative communications during emergency response. Washington, DC: NRT Response Subcommittee Workgroup.

US Nuclear Regulatory Commission (NRC). 2004. The technical basis for the NRC's guidelines for external risk communication. Washington, DC: Office of Nuclear Regulatory Research. 
Willey, Malcolm and Rice, Stuart A. 1933. The agencies of communication. IN: Presidential committee on social trends (ed.). Recent social trends in the United States. Volume 1. New York: McGraw Hill.

\section{Articles, essays and papers presented}

Ahearne, John F. 2000. Intergenerational issues regarding nuclear power, nuclear waste, and nuclear weapons. Risk analysis. 20(6), pp763-770.

Alexander, David. 2005. Toward the development of a standard in emergency planning. Disaster prevention and management. 14(2), pp158-175.

Austin, Erica W. and Pinkleton, Bruce E. and Dixon, Andrea. 2000. Barriers to public relations programme research. Journal of public relations research. 12(3), pp235-253.

Baker, Sherry and Martinson, David L. 2002. The TARES test: five principles for ethical persuasion. Journal of mass media ethics. 16(2), pp148-175.

Ball, David J. 2002. Environmental risk assessment and the intrusion of bias. Environment international. 28, pp529-544.

Ball, David J. 2005. Nuclear waste: consult widely, decide wisely. The chemical engineer. September, pp25-28.

Ball, David J. 2006. Deliberating over Britain's nuclear waste. Journal of risk research. 9(1), pp1-11.

Becker, Boris W. and Connor, Patrick E. 1979. Personal values of the heavy user of mass media. Journal of advertising. 21, pp37-43.

Bertrand, Robert and Lajtha, Chris. 2002. A new approach to crisis management. Journal of contingencies and crisis management. 10(4), pp181-191.

Boin, Arjen and Lagadec, Patrick. 2000. Preparing for the future: critical challenges in crisis management. Journal of contingencies and crisis management. 8(4), pp185191. 
Bostrom, Ann and Lofstedt, Ragnar E. 2003. Communicating risk: wireless and hardwired. Risk analysis. 23(2), pp241-248.

Bouwer, Laurens and Crompton, Ryan and Faust, Eberhard and Hoppe, Peter and Pielke, Roger. 2007. Confronting disaster losses. Science. 318, p753.

Broom, Glen M. and Casey, Shawna and Ritchey, James. 1997. Toward a concept and theory of organisation - public relations. Journal of public relations research. 9(2), pp83-98.

Brown, Timothy S. 2003. Powerful crisis communication lessons. Public relations quarterly. 48(4), pp21-25.

Bryant, Jennings. 2004. Critical communication challenges for the new century. Journal of communication. 10, pp389-401.

Burling Wynona K. and Hyle, Adrienne E. 1997. Disaster preparedness planning: policy and leadership issues. Disaster prevention and management. 6(4), pp234-244.

Carrington, Judith. 1992. Establishing a more strategic role in PR practice: why, how and when? Public relations quarterly. 37(1), pp45-47.

Chess, Caron and Burger, Joanna and Hughes-McDermott, Melanie. 2004. Speaking like a state: environmental justice and fish consumption advisories. Society and natural resources. 18, pp267-278.

Chess, Caron and Calia, Jeff and O’Neill, Karen. 2004. Communication triage: an Anthrax case study. Biosecurity and bioterrorism: biodefense strategy, practice, and science. 2(2), pp106-111.

Chong, Mark. 2006. A crisis of epidemic proportions: what communication lessons can practitioners learn from the Singapore SARS crisis. Public relations quarterly. Spring, pp6-11.

Comfort, Louise K. 1994. Risk and resilience. Journal of contingencies and crisis management. 2(3), pp174-188.

Comfort, Louise K. and Sungu, Yesim and Johnson, David. 2001. Complex systems in crisis: anticipation and resilience in dynamic environments. Journal of contingencies and management. 9(3), pp144-158. 
Conkey, Howard. 2004. National crisis communication arrangements for agricultural emergencies. The Australian journal of emergency management. Autumn, pp43-46.

Connelly, Nancy A. and Knuth, Barbara A. 1998. Evaluating risk communication: examining target audience perceptions about four presentation formats for fish consumption health advisory information. Risk analysis. 18(5), pp649-659.

Cottrell, Alison. 2005. Sometimes it's a big ask, but sometimes it's a big outcome: community participation in flood mitigation. The Australian journal of emergency management. Autumn, pp27-31.

Covello, Vincent and Wojtecki, Joseph G. and Hyde, Richard C. 2001. Risk communication, the West Nile virus epidemic, and bio-terrorism: responding to the communication challenges posed by the intentional or unintentional release of a pathogen in an urban setting. Journal of urban health: bulletin of the New York academy of medicine. 78(2), pp382-391.

Dake, Karl. 1991. Orienting dispositionsin the perception of risk: an analysis of worldviews and cultural biases. Journal of cross-cultural psychology. 22(1), pp61-82.

Daugherty, Emma. 2003. Strategic planning in public relations: a matrix that ensures tactical soundness. Public relations quarterly. 48(1), pp21-26.

Dyer, Samuel C. 1995. Getting people into the crisis communication plan. Public relations quarterly. 40(3), pp38-41.

Ehling, William P. 1985. Application of decision theory in the construction of a theory of public relations management. Public relations research and education. 2(1), pp233-238.

Elliott, Dominic and Smith, Denis. 1993. Football stadia disasters in the United Kingdom: learning from tragedy. Industrial and environmental crisis quarterly. 7(3), 205-229.

Elliott, Dominic and Smith, Denis and McGuinness, Martina. 2000. Exploring the failure to learn: overcoming the barriers to learning. Crisis review of business. 21(3), p17. 
Fahey, Christine. 2003. Working with communities to build social capital: reflecting on old and new thinking about volunteers. Australian journal of emergency management. Winter, pp12-17.

Ferguson, Mary A. 1984. Building theory in public relations: inter-organisational relationships. Paper presented at: Association for education in journalism and mass communication. May 1984. Gainesville, Florida.

Fischer, Henry W. 1999. Enhancing disaster mitigation planning and response through the use of cyberspace: suggestions and issues to consider. Journal of contingencies and crisis management. 7(1), pp48-54.

Fischer, Henry W. 2002. Terrorism and 11 September 2001: does the 'behavioural response to disaster' model fit? Disaster prevention and management. 11(2), pp123127.

Fischhoff, Baruch. 1995. Risk perception and communication unplugged: twenty years of process. Risk analysis. 15(2), pp137-144.

Fischhoff, Baurach and Slovic, Paul and Lichtenstein, Steve. 1982. Lay foibles and expert fables in judgements about risk. American statistician. 36, pp240-255.

Fitzgerald, John. 2000. Education and young people - forces for change? The Australian journal of emergency management. Spring, p1.

Flynn, Dennis. 2003. Bringing a crisis under control. Emergency services Ireland. 10, pp85-88.

Fordham, Maureen. 1998/99. Participatory planning for flood mitigation: models and approaches. Australian journal of emergency management. Summer, pp27-34.

Freitag, Alan R. 1998. How to measure what we do. Public relations quarterly. 43(2), pp42-47.

Frosdick, Steve. 1995. Organisational structure, culture and attitudes to risk in the British stadia safety industry. Journal of contingencies and crisis management. 3(1), pp43-58.

Geis, David. 2000. By design: the disaster-resistant and quality-of-life community. Natural hazards review. 1(3), pp151-160. 
Gherardi, Silvia. 1998. A cultural approach to disasters. Journal of contingencies and crisis management. 6(2), pp80-83.

Goldenbeld, Charles and Levelt, Peter B. and Heidstra, Jelle. 2000. Psychological perspectives on changing driver attitude and behaviour. Recherche transports securite. 67 , pp65-81.

Granger, Ken. 2000. An information infrastructure for disaster management in Pacific island countries. Australian journal of emergency management. Autumn, pp20-32.

Grunig, James E. and Grunig, Larissa A. 2000. Public relations in strategic management and strategic management of public relations: Theory and evidence from the IABC excellence project. Journalism studies. 1(2), pp303-323.

Gustafson, Per E. 1998. Gender differences in risk perception: theoretical and methodological perspectives. Risk analysis. 18(6), pp805-811.

Hale, Joanne E. 1997. A layered communication architecture for the support of crisis response. Journal of management information systems. 14(1), pp235-255.

Hale, Joanne and Dulek, Ronald E. and Hale, David P. 2005. Crisis response communication challenges. Journal of business communication. 42(2), pp112-134.

Handmer, John and Parker, Denis. 1991. British disaster planning and management: an initial assessment. Disasters. 15, pp303-317.

Heath, Robert. 1995. The Kobe earthquake: some realities of strategic management of crises and disasters. Disaster prevention and management. 4(5), pp11-24.

Helsloot, Ira and Ruitenberg, Arnout. 2004. Citizen response to disasters: a survey of literature and some practical implications. Journal of contingencies and crisis management. 12(3), pp98-111.

Heneghan, Grace. 2006. Emergency plans under new framework. Emergency services Ireland. 23(1), pp15-19.

Heneghan, Grace. 2007. In the event of an emergency. Emergency services Ireland. 27(1), pp107. 
Herrero, Alfonso G. and Pratt, Cornelius B. 1996. An integrated symmetrical model for crisis communications management. Public relations research. 8(2), pp79-105.

Hills, Alice E. 1994. Coordination and disaster response in the United Kingdom. Disaster prevention and management. 3(1), pp66-71.

Hocke, Irene and O'Brien, Arthur. 2003. Strengthening the capacity of remote indigenous communities through emergency management. Australian journal of emergency management. Summer, pp62-69.

Hon, Linda C. 1998. Demonstrating effectiveness in public relations: goals, objectives and evaluation. Journal of public relations research. 10(2), pp103-135.

Huang, Yi-Hui. 2004. Is symmetrical communication ethical and effective. Journal of business ethics. 53, pp333-352.

Hughes, Lewis and Henry, John. 2003. Volunteers as a learning bridgehead to the community. Australian journal of emergency management. Winter, pp25-31.

Hutton, James G. 1999. The definitions, dimensions, and domain of public relations. Public relations review. 25, pp199-214.

Jacobs, Gerard A. 1990. Lessons from the aftermath of Flight 232: practical considerations for the mental health profession's response to air disasters. American psychologist. 45(12), p1332.

Jones, Charlotte M. 1999. Shifting sands: women, men, and communication. Journal of communication. 3, pp148-155.

Kinney, Guglielmo Aimee and Leschine, Thomas M. 2002. A procedural evaluation of an analytic-deliberative process: the Columbia River comprehensive impact assessment. Risk analysis. 22(1), pp83-100.

Kirkwood, Alan. 1999. Discounting the unexpected: the limitation of isomorphic thinking. Risk management: an international journal. 1, pp33-44.

Kulling, Per E. and Holst, Jonas E. 2004. Educational and training systems in Sweden for prehospital response to acts of terrorism. Pre-hospital and disaster medicine. 18(3), pp184-188. 
Kweit, Mary G. and Kweit, Robert W. 1984. The politics of policy analysis: the role of citizen participation in analytic decision-making. Policy studies review. 3(2), pp3233.

Ledingham, John A. 2000. Guidelines to building and maintaining strong organisational-public relationships. Public relations quarterly. 45(3), pp44-46.

Levick, Richard S. and Smith, Larry. 2005. In the wake of catastrophe...warring words and duelling message points. Public relations quarterly. Fall, pp5-7.

Lindeborg, Richard A. 1994. Excellent communication. Public relations quarterly. 39(1), pp5-11.

Lindenmann, Walter K. 1998/99. Measuring relationships is key to successful public relations. Public relations quarterly. 43(4), pp18-24.

Lofstedt, Ragnar E. and Ikeda, Saburo and Thompson, Kimberly M. 2000. Risk management across the globe: insights from a comparative look at Sweden, Japan, and the United States. Risk analysis. 20(2), pp157-161.

Maloney, Kevin. 1997. Teaching organisational communication as public relations in UK universities. Corporate communication: an international journal. 2, pp138-142.

Marra, Francis J. 1998. The importance of communication in excellent crisis management. Australian journal of emergency management. Spring, pp6-9.

McEntire, David A. 2005. Why vulnerability matters. Disaster prevention and management. 14(2), pp206-222.

Mitchell, Tony H. 1986. Coping with a corporate crisis. Canadian business review. 13, pp17-20.

Mitroff, Ian and Harrington, Katharine and Gai, Eric. 1996. Thinking about the unthinkable. Across the board. 33(8), pp44-48.

Morrisey, Shirley A. and Reser, Joseph P. 2003. Evaluating the effectiveness of psychological preparedness advice in community cyclone preparedness materials. Australian journal of emergency management. Summer, pp46-60. 
Murphy, Priscilla. 1991. The limits of symmetry: a game theory approach to symmetric and asymmetric public relations. Journal of public relations research. 3(1), pp115-131.

Murray, Liam G. and Greenwood, Tony K. 1993. Information to the public following a nuclear emergency: a role for local authorities. Disaster prevention and management. 2(2), pp51-60.

National Academy of Sciences. 2002. Alerting America: effective risk communication. Paper presented at the 6th 'Natural disasters roundtable forum'. October $31^{\text {st }}$ 2002. Washington, DC.

Nielsen, Samuel and Lidstone, John. 1998. Public education and disaster management: is there any guiding theory. Australian journal of emergency management. Spring, pp14-19.

Nohrstedt, Stig A. 2000. Communication challenges in connection with catastrophes and states of emergency: a review of the literature. Nordicom review. 21(2), pp137149.

O'Brien, Geoff and Read, Paul. 2005. Future UK emergency management: new wine, old skin? Disaster prevention and management. 14(3), pp353-361.

Palenchar, Michael P. and Heath, Robert L. 2002. Another part of the risk communication model: analysis of communication processes and message content. Journal of public relations research. 14(2), pp127-158.

Palenchar, Michael P. and Heath, Robert L. 2007. Strategic risk communication: adding value to society. Public relations review. 33, pp120-129.

Parry-Giles, Shawn J. 1994. Rhetorical experimentation and the Cold War, 19471953: the development of an internationalist approach to propaganda. Quarterly journal of speech, 80, pp448-467.

Payne, Christopher F. 1999. Contingency plan exercises. Disaster prevention and management. 8(2), pp111-117.

Pearson, Christine M. and Mitroff, Ian I. 1993. From crisis prone to crisis prepared: a framework for crisis management. The executive. 7(1), pp48-59. 
Perry, Ronald W. 2003. Incident management systems in disaster management.

Disaster prevention and management. 12(5), pp405-412.

Pieczka, Magda. 2000. Objectives and evaluation in public relations work: what do they tell us about expertise and professionalism? Journal of public relations research. 12(3), pp211-233.

Plowman, Kenneth. 2005. Conflict, strategic management, and public relations. Public relations review. 31, pp131-138.

Porfiriev, Boris. 1999. Recovery policy in crisis areas: the risk management concept versus normative and administrative approaches to rehabilitation of the radioactively contaminated areas in the former Soviet Union. Journal of contingencies and crisis management. 7(2), pp76-85.

Putnam, Linda I. 2001. Shifting voices, oppositional discourse, and new visions for communication studies. Journal of communication. 3, pp38-51.

Quarantelli, Enrico L. 1984. Organisational behaviour in disasters and implications for disaster planning. Monographs of national emergency training centre. 1(2), pp131.

Quarantelli, Enrico L. 1995. What is a disaster? International journal of mass emergencies and disasters. 13(3), pp221-229.

Reynolds, Barbara and Seeger, Matthew W. 2005. Crisis and emergency risk communication as an integrative model. Journal of health communication. 10, pp4355.

Richardson, Brian. 1994. Socio-technical disasters: profile and prevalence. Disaster prevention and management. 3(3), pp59-80.

Riley, Julie and Meadows, Jack. 1997. The role of information in disaster planning: a case study approach. Disaster prevention and management. 6(5), pp349-355.

Rogers, George O. 1989. Communication of emergency warning: a cyclical process. Disaster management. 1(4), pp23-28. 
Rohrmann, Bernd. 2000. Critical assessment of information on bushfire preparedness for residents. Australian journal of emergency management. Autumn, pp14-19.

Rose, Pat. 2002. The times they are a changing... and so should PR training. Public relations quarterly. 47(1), pp14-17.

Rowe, Gene and Wright, George. 2001. Differences in expert and lay judgements of risk: myth or reality. Risk analysis. 21(2), pp341-356.

Salter, John. 1996. Towards a better disaster management methodology. Australian journal of emergency management. Autumn, pp8-16.

Sandman, Peter M. 1992. Responding to community outrage: strategies for effective risk communication. Fairfax, VA: American Industrial Hygiene Association.

Sandman, Peter M. 2003. Four kinds of risk communication. The synergist. April, pp26-27.

Scanlon, Joseph. 1997. Human behaviour in disaster: the relevance of gender. Australian journal of emergency management. Summer, pp2-7.

Scanlon, Joseph. 2006. Dealing with the tsunami dead: unprecedented international cooperation. Australian journal of emergency management. Summer, pp57-62.

Schoemaker, Paul J. H. 1993. Multiple scenario development: its conceptual and behavioral foundation. Strategic management journal. 14(3), pp93-213.

Schoenberg, Alan. 2005. Do crisis plans matter? a new perspective on leading during a crisis. Pubic relations quarterly. Spring, pp2-6.

Sen, Falguni and Egelhoff, William G. 1991. Six years and counting: learning from crisis management at Bhopal. Public relations review. 17(1), pp69-83.

Shaluf, Ibrahim and Ahmadun, Fakharu'l-razi and Said, Aini Mat. 2003. A review of disaster and crisis. Disaster prevention and management. 12 (1), pp24-32.

Shaw, Kieron. 2005. Getting leaders involved in communication strategy. Strategic communication management. 9(6), pp14-27. 
Shaw, Rajib and Sinha, Ravi. 2003. Towards sustainable recovery: future challenges after the Gujarat earthquake, India. Risk management: an international journal. 5(4), pp35-51.

Shaw, Rajib and Shiwaku, Koichi and Chandra-Kandel, Ram and Narayan-Shrestha, Surya and Mani-Dixit, Amod. 2004. Linking experience, education, perception and earthquake preparedness. Disaster prevention and management. 13(1), pp39-49.

Shaw, Richard. 2001. Don't panic: behaviour in major incidents. Disaster prevention and management. 10(1), pp5-9.

Simpson, Andrea L. 1992. Ten rules of research. Public relations quarterly. 37(2), pp27-28.

Sjoberg, Lennart. 1998. World-views, political attitudes and risk perception. Risk: health, safety and environment. 9(1), pp137-152.

Sjoberg, Lennart. 2000. Factors in risk perception. Risk analysis. 20(1), pp1-11.

Stewart, Michael. 1989. Surviving the horror. Community care. February, pp26-28.

Steyn, Benita and Green, Mateboho and Grobler, Anske. 2001. Strategic management roles of the corporate communication function: are they being played in the government departments? Paper presented at: $8^{\text {th }}$ international public relations research symposium: politics of communication and communication of politics. July 2001. Lake Bled, Slovenia.

Sturges, David L. 1994. Communicating through crisis: a strategy for organisational survival. Management communication quarterly. 7(3), pp297-316.

't Hart, Paul and Heyse, Liesbet and Boin, Arjen. 2001. New trends in crisis management practice and crisis management research: setting the agenda. Journal of contingencies and crisis management. 9(4), pp181-188.

Temple, Richmond K. 2002. Setting CLEAR goals: the key ingredient to effective communication planning. Public relations quarterly. 47(2), pp32-34.

Toft, Brian. 1992. The failure of hindsight. Disaster prevention and management. 1(3), pp48-60. 
Trim, Peter R. 2004. An integrative approach to disaster management and planning. Disaster prevention and management. 13(3), pp218-225.

Trumbo, Craig W. and McComas, Katherine A. 2003. The function of credibility in information processing. Risk analysis. 23(2), pp343-353.

Tumelty, David. 1990. Life after disaster hits. Social work today. 21(22), 14-16.

Turner, Barry A. 1997. Rethinking organisations: organisational learning in the nineties. Paper presented at: The European foundation for management development (EFMD) research conference. October 1991. Palermo, Italy.

Ulmer, Robert and Seeger, Matthew W. and Sellnow, Timothy. 2007. Post-crisis communication and renewal: expanding the parameters of post-crisis discourse. Public relations review. 33, pp130-134.

Van Houts, Paul. 1996. The SWOT analysis: another planning tool for emergency management. The Australian journal of emergency management. Spring, pp20-22.

Wahl-Jorgensen, Karin. 2004. How not to found a field: new evidence on the origins of mass communication research. Journal of communication. 10, pp547-564.

Waring, Stephen C. and Brown, Bruce J. 2005. The threat of communicable diseases following natural disasters: a public health response. Disaster management and response. 3(2), pp41-47.

Webster, Peter J. 1990. Strategic corporate public relations: what's the bottom line? Public relations journal. 46(2), pp18-21.

Wildavsky, Aaron and Dake, Karen. 1990. Theories of risk perception: who fears what and why. Daedalus. 119(4), pp41-60.

\section{Dissertations and Academic papers}

Bowman, Valerie. 2000. What purpose should public consultation serve in emergency planning. Submitted in partial fulfillment of a Diploma in Management Studies, University of Northumbria at Newcastle Business School. 
Connors, Paul. 2003. In the line of duty : an analysis of the quality and efficacy of bereavement support provided by the defence forces to the families of Irish soldiers who die in the line of duty. Submitted in partial fulfillment of a Masters in Communications and Cultural Studies, Dublin City University.

Scarman Centre. 2004. Module 2. Study texts available to off-campus students undertaking: MSc in Security and Risk Management. Texts received from a former student of this course in 2004. Leicester: University of Leicester. Permission received to cite text from Ms Tracey Doddman, Teaching Fellow in Department of Criminology, University of Leicester.

\section{Print media}

Berger, Sebastien. 2004. Scribbled notes help victims and loved ones make contact. Daily Telegraph. December 29, p11.

Bird Flu. 2006. Editorial. Sunday Telegraph. April 9, p20.

Brady, Tom. 2006. Chemical Willie goes into crisis mode... silently. Irish Independent. November 17, p3.

Chakravarti, Sunetra. 2005. Boy found alive in a coconut tree. The Times. January 8 , p4.

Chan, Sewell. 2005. Portrait of Mississippi victims: safety of home was a mirage. New York Times. September 27, p5.

Cloonan, Clifford. 2008. New horrors as devastated towns finally reached. Irish Times. May 16, p13.

Connolly, Shaun. 2008. A national emergency... time for Willie and the website. Irish Examiner. April 15, p4.

Cusack, Jim. 2002. Leaflet outlines State plan on nuclear fall-out. Irish Times. March $8, \mathrm{p} 1$.

Cusack, Jim. 2004. Kissing our arses goodbye. Sunday Independent: Life. September 5, pp25-28. 
Dejevsky, Mary. 2005. How hurricane plunged first world city into the third world overnight. Irish Independent. September 1, p20.

Delevan, Richard. 2007. Old media vs. new media. Business and Finance. November 9-22, p68.

Downes, John. 2008. Most people in Ireland know little about Bebo, survey finds. Irish Times. February 20, p10.

Drummond, Andrew. 2005. Experts ignored e-mail warning. The Times. January 7, p6.

Eggen, Dan. 2006. NY releases recordings of 9/11 calls made on September $11^{\text {th }}$. Washington Post. April 1, pA03.

Fahy, Declan and Donnellan, Eithne. 2001. Martin's assurance on iodine supply incorrect. Irish Times. September 28, p8.

Flanagan, Ben. 2005. Don't let disaster get in the way of a real story. Guardian. December 18, p2.

Fournier, Ron. 2005. Playing the blame game: Hurricane Katrina victims failed by US leaders. Irish Examiner. September 3, p10.

Gold, Scott. 2005. Refugees forced to endure appalling conditions. Irish Times. September 2, p10.

Grady, Denise. 2005. Training is first task in Angola outbreak. New York Times. April 11, p7.

Gross, Jane. 2005. Aging, frail and refugees from the hurricane. New York Times. September 18, p3.

Hardiman, Orla. 2007. Loss of confidence in public health sector has serious consequences. Irish Times. July 27, p16.

Harrison, David and Copping, Jasper. 2006. So, is this what the government meant by 'the best-prepared nation in the world' for bird flu? Sunday Telegraph. April 9, p13. 
Hidden killer. 2006. Economist. February 11, p32.

Hill, Amelia. 2002. I won't let Chernobyl happen here. Guardian. April 21, p8.

Horgan, Edward. 2001. Time to face the nightmare scenario. Irish Times. October 2, p16.

Horgan, John. 2002. An Irishman's diary. Irish Times. February 9, p17.

Irish Times. 2001(a). Braving the PR fallout of nuclear plan fiasco. Ir. Times.

November 11, p5.

Irish Times. 2001(b). Task force sees no threat, but continues to develop a 'practical' national emergency plan. Ir. Times. December 17, p11.

Irish Times. 2001(c). Nuclear attack is not covered by State plan. Ir. Times. October $2, \mathrm{p} 4$.

Leahy, Pat. 2008. Cowen can prosper off pigs' backs. Sunday Business Post.

December 14, p8.

Lipton, Eric. 2007. Aide loses job over fake news conference. New York Times. October 30, p2.

Mansnerus, Laura. 2006. The week; between floods, talk, not action, on solutions. New York Times: magazine. July 9, p12.

McDonald, Frank. 2007. Academy warns of more severe flooding in Ireland. Irish Times. August 30, p3.

McGuire, Bill. 2004. We need a warning system too. Guardian. December 30, p10.

McWilliams, Brendan. 2004. Detecting tsunamis: a problem for geophysicists. Irish Times. December 27, p10.

McWilliams, Brendan. 2006. Natural disasters sharpen the mind. Irish Times. March 23, p25.

McWilliams, Brendan. 2007. A blow-by-blow account of tornadoes. Irish Times. August 24, p26. 
Melia, Paul. 2007. We are now a rainbow nation. Irish Independent. November 5, p17.

Monbiot, George. 2005. We care, but only when we know. Guardian. January 15, p15.

Mountain tsunami. 2005. Special report. Economist. October 15, pp25-27.

Nussbaum, Bruce. 2005. The next big one. Business week. September 19, pp32-40.

O'Brien, Breda. 2006. The Garda could learn from Catholic Church. Irish Times. August 26, p14.

O'Connor, Alison. 2001. Emergency plans for nuclear attack updated. Irish Times. September 27, p1.

O'Halloran, Marie. 2005. Ireland 'ready' for any emergency. Irish Times. March 8, p5.

Owen, Paul. 2005. UN warning over tents for earthquake survivors. Guardian.

December 2, p4.

Padgett, Tim. 2005. Can New Orleans do better. Time Magazine. October 24, pp1821.

Reid, Liam. 2005. State watches about 24 Islamic suspects. Irish Times. July 14, p6.

Siggins, Lorna. 2007. Ireland to develop early-alert system for tsunami. Irish Times. February 15, p5.

Sipress, Alan. 2005. Indonesia neglected 'bird flu' until too late, experts say.

Washington Post. 20 October, pA01.

Staunton, Denis. 2005. Police warn campaign of terror may not be over. Irish times. July $8, \mathrm{p} 1$.

Tyrrell, Fiona. 2006. Emergency cover. Irish Times: health supplement. November 7, p8. 
Walsh, Bryan. 2005. A wing and a prayer. Time Magazine. October 17, pp26-33.

\section{Electronic media}

Adland. 2005. Asian ad. pulled due to Asian tsunami. Advertising news [Online]. Available from: http://commercial-archive.com/116564.php [Accessed 14 January 2008].

Boucher-Hayes, Philip. 2005. Report Thailand. Today with Pat Kenny. Radio Teilifis Eireann (RTE) radio. 6 January 2005.

Boucher-Hayes, Philip. 2008. Your major emergency handbook. Drivetime. Radio Teilifis Eireann (RTE) radio. 25 April 2008.

Breakingnews. 2004. Death toll rises [Online]. Available from http://www.breakingnews.ie [Accessed 31 December 2004].

British Broadcasting Corporation (BBC). 2005. London rocked by terror attacks. $B B C$ News [Online]. Available from http://news.bbc.co.uk/2/hi/uk_news/england/london/4659093.stm [Accessed 7 June 2008].

British Broadcasting Corporation (BBC). 2006. Clooney begs UN to act on Darfur. BBC News [Online]. 14 September 2006. Available from http://news.bbc.co.uk/2/hi/americas/5347660.stm [Accessed 5 January 2008].

British Broadcasting Corporation (BBC). 2007. King's Cross radio faults remain. $B B C$ News [Online]. 18 November 2007. Available from http://news.bbc.co.uk/1/hi/uk/7098306.stm [Accessed 6 January 2008].

British Broadcasting Corporation (BBC). 2008(a). Burma death toll jumps to 78,000. BBC News [Online]. Available from http://news.bbc.co.uk/2/hi/asiapacific/7405260.stm [Accessed 27 May 2008].

British Broadcasting Corporation (BBC). 2008(b). Connecting in a crisis. $B B C$ News [Online]. Available from www.bbc.co.uk/connectinacrisis/index.shtml [Accessed 17 February 2008]. 
British Broadcasting Corporation (BBC). 2008(c). China earthquake toll jumps again. BBC News [Online]. Available from http://news.bbc.co.uk/2/hi/asiapacific/7416035.stm[Accessed 27 May 2008].

Cowen, Kevin. 2005. Interview. Morning Ireland. Radio Teilifis Eireann (RTE) radio. 30 August 2005.

Federal Emergency Management Agency (FEMA). 2006. Getting ready for disasters. DVD. Washington, DC: FEMA.

Ganley, Declan. 2008. Interview. Interview with Eamon Dunphy. . Radio Teilifis Eireann (RTE) radio. 14 June 2008.

Mullooly, Ciaran. 2008. 1,400 laid off after pork recall. Nine O'Clock News. Radio Teilifis Eireann (RTE) television. 8 December 2008.

\section{Websites}

Cabinet Office. 2008. About the Cabinet Office [Online]. Available from http://www.cabinetoffice.gov.uk [Accessed 9 June 2008].

Citizen Corps. 2008. Are you ready [Online]. Available from http://www.citizencorps.gov/ready/ [Accessed 10 February 2008].

Civil Contingencies Secretariat (CCS). 2007. Local response gateway [Online]. Available from http://www.ukresilience.info/ccs/local_response_gateway.aspx [Accessed 30 October 2007].

Craig, Bob. 2008. Theory [Online]. Available from http://www.colorado.edu/communication/meta-discourses/theory.htm [Accessed 5 May 2008].

Department of Defence. 2007. Office of emergency planning [Online]. Available from http://www.defence.ie [Accessed 6 November 2007].

Department of the Environment, Heritage and Local Government (DEHLG). 2007. Emergency planning [Online]. Available from www.environ.ie [Accessed 18 November 2007]. 
Federal Emergency Management Agency (FEMA). 2008(a). Homepage [Online]. Available from http://www.fema.gov/ [Accessed 7 January 2008].

Federal Emergency Management Agency (FEMA). 2008(b). FEMA for kids [Online]. Available from http://www.fema.gov/kids/ [Accessed 17 February 2008].

HM Government. 2006. Preparing for emergencies: what you need to know [Online]. Available from http://www.preparingforemergencies.gov.uk/ [Accessed 18 February 2008].

Home Office. 2008. Recovery: an emergency management guide[Online]. Available from http://www.coordination.gov.uk/upload/assets/www.ukresilience.info/recovery.pdf [Accessed 26 January 2008].

International Nuclear Safety Center (INSC). 2007. Database [Online]. Available from http://www.insc.anl.gov/ [Accessed 3 November 2007].

Mercali Intensity Scale. 2008. Homepage [Online]. Available from http://www.abag.ca.gov/bayarea/eqmaps/doc/mmi.html [Accessed 7 January 2008].

Office of Emergency Planning (OEP). 2007. Homepage [Online]. Available from http://www.emergencyplanning.ie/ [Accessed 13 November 2007].

Office of Public Works (OPW). 2008. Flooding: plan, prepare, protect [Online]. Available from http://flooding.ie/ [Accessed 8 February 2008].

Oregon State University. 2008. [Online]. Available from http://oregonstate.edu/instruct/theory/contexts.html [Accessed 8 May 2008].

Radiological Protection Institute Ireland (RPII). 2007. Homepage [Online]. Available from http://www.rpii.ie [Accessed 12 November 2007].

Sandman, Peter M. 2002. Crisis communication versus risk communication. The Peter Sandman risk communication website [Online]. Available from http://www.psandman.com/gst2002.htm [Accessed 30 April 2006]. 
Sandman, Peter M. 2004(a). Crisis communication: a very quick introduction. The Peter Sandman risk communication website [Online]. Available from http://www.psandman.com/col/crisis.htm [Accessed 7 January 2008].

Sandman, Peter M. 2004 (b). Crisis communication III: involving the public. The Peter Sandman risk communication website [Online] Available from http://www.psandman.com.htm. [Accessed 7 January 2008].

Sandman, Peter M. 2005. Katrina: hurricanes, catastrophes, and risk communication. The Peter Sandman risk communication website [Online]. Available from http://www.psandman.com/col/katrina.htm [Accessed 7 January 2008].

Sandman, Peter M. 2008. Homepage. The Peter Sandman risk communication website [Online]. Available from http://www.psandman.com/ [Accessed 10 May 2008].

Sandman, Peter M. and Lanard, Jody. 2003. Fear of fear: the role of fear in preparedness... and why it terrifies officials. The Peter Sandman risk communication website [Online]. Available from http://www.psandman.com/col/fear.htm [Accessed 7 January 2008].

Sandman, Peter M. and Lanard, Jody. 2005. Tsunami risk communication: warnings and the myth of panic. The Peter Sandman risk communication website [Online]. Available from http://www.psandman.com/col/tsunamil.htm [Accessed 7 January 2008].

Sandman, Peter M. and Lanard, Jody. 2007. Empathy in risk communication. The Peter Sandman risk communication website [Online]. Available from http://www.psandman.com/ col/empathy.htm [Accessed 7 January 2008].

Siemens. 2008. New public safety radio system for Norway. Innovation news [Online]. Available from http://w1.siemens.com/innovation/en/news_events/innovationnews/innovationne ws_articles/information_communications/newpublicsafetyradiosystemfornorway. htm [Accessed 5 January 2008].

SOS Alarm. 2007. For a safer society [Online]. Available from http://www.sosalarm.se [Accessed 13 August 2007]. 
Swedish Emergency Management Agency (SEMA). 2007. The hub of Sweden's crisis management system [Online]. Available from http://www.

Krisberedskapsmyndigheten.se [Accessed 11 August 2007].

Swedish Ministry of Defence. 2008. Areas of responsibility [Online]. Available from http://www.sweden.gov.se/sb/d/2060 [Accessed 17 February 2008].

Thurston County. 2006. Telephone Alert System [Online]. Available from http://www.co.thurston.wa.us/em/Phone/index.htm [Accessed 5 January 2008].

UK Resilience. 2005. Communicating risk. [Online]. Available from http://www.ukresilience.info/upload/assets/www.ukresilience.info/communicatingris k.pdf_[Accessed 7 January 2008].

UK Resilience. 2007. Warning and informing the public. Emergency Preparedness [Online]. Available from http://www.ukresilience.info/preparedness/warningandinforming.aspx [Accessed 7 January 2008].

United Nations International Strategy for Disaster Reduction (UNISDR). 2007. Disaster statistics [Online]. Available from http://www.unisdrorg [Accessed 2 November 2007].

University of Twente. 2008. Communication theories [Online]. Available from http://www.tcw.utwente.nl/theorieenoverzicht/ [Accessed 5 May 2008].

US Department of Homeland Security (DHS). 2007. Ready America [Online]. Available from http://www.ready.gov/america/index.html [Accessed 6 September 2007].

US Department of Homeland Security (DHS). 2008. Homepage [Online]. Available from http://www.the DHS.gov [Accessed 17 February 2008].

World Health Organisation (WHO). 2008. Glossary. Some terms used in toxicology and chemical safety [Online]. Available from http://www.bio.hw.ac.uk/edintox/glossall.htm [Accessed 16 January 2008]. 


\section{Interviews and personal communication}

For details on all interviews and personal communication see Appendix A. 


\section{APPENDICES}




\section{APPENDIX A - DETAILS OF THOSE WHO PARTICIPATED IN THE RESEARCH}

Detailed in-depth and elite interviews were conducted with key individuals involved in major emergency management in Ireland, while comprehensive selfadministered questionnaires were completed by three international major emergency communication practitioners.

\section{$\underline{\text { Ireland }}$}

\section{In-depth and elite}

Minister Willie O'Dea TD - Minister for Defence and Chairperson of the Government Task Force on Major Emergency Management. The interview was conducted with Minister O'Dea in the Minister's office in Leinster House on January $23^{\text {rd }}, 2008$. The interview lasted approximately one hour. Present also on this occasion was Mr Derek Mooney, Political Advisor to the Minister, who made some relevant comments that were noted.

Mr Martin Heraghty - Assistant Secretary Corporate Affairs, Department of Agriculture, Fisheries and Food (DAFF). Mr Heraghty is also responsible for leading major emergency planning, preparedness and response in DAFF. He has had previous experience of managing the response to 'foot-and-mouth' in 2001. The interview was conducted with Mr Heraghty in his office in the Department of Agriculture, Kildare Street, Dublin on November $19^{\text {th }}, 2007$. The interview lasted one hour and fifteen minutes.

Mr Tony Boland - Assistant Secretary, Department of Environment, Heritage and Local Government (DEHLG). Mr Boland has responsibility for overseeing major emergency management for DEHLG. The interview took place in $\mathrm{Mr}$ Boland's office in the Department of the Environment, Custom House, Dublin, on October $30^{\text {th }}, 2007$, and lasted approximately one hour and fifteen minutes. 
Present also on this occasion was Ms Celina Barrett, Principal Officer, DEHLG, who made some relevant comments that were noted.

Lieutenant General Pat Nash - former Deputy Chief of Staff (operations). An interview took place with Lieutenant General Nash in his office at Defence Forces Headquarters, Infirmary Road, Dublin on September $26^{\text {th }}, 2007$, a number of days prior to taking up his new appointment as Operational Commander of the EU Force in Chad. Lieutenant General Nash provided an insight into the Defence Forces' role in major emergency management.

In-depth

Mr Brian Spain - Principal Officer Department of Defence and Head of the Office of Emergency Planning (OEP). As Chair of the Inter-Departmental Working Group, Mr Spain has a central role in major emergency planning and preparedness in Ireland. The interview was conducted in Mr Spain's Office in the Department of Defence on Infirmary Road on December $12^{\text {th }}, 2007$. The interview lasted approximately one hour and thirty minutes.

Mr Gavin Maguire - Assistant National Director in Population Health, Health Services Exectutive (HSE), the Director of major emergency planning for the HSE, and a member of the National Steering Group. The interview with $\mathrm{Mr}$ Maguire was conducted in his office in the Civic Offices, Bray, Co. Wicklow on December $10^{\text {th }}, 2007$. The interview took approximately one hour.

Mr Pat O'Riordan - A further emergent interview was conducted with Mr Pat O'Riordan of the HSE Mid-West region. Mr O'Riordan is a member of both the National Steering Group and the National Working Group, and he wrote the first substantial book on major emergency planning in Ireland in 1992. The interview with Mr O'Riordan took place in his office in Limerick on January $17^{\text {th }}, 2008$ and lasted approximately one hour.

Mr Eoghan O’Neachtain - Government Press Secretary, Department of an Taoiseach. Mr O'Neachtain is also the head of Government Information Services and Chair of the Emergency Planning Media Unit (EPMU). The interview was 
conducted with Mr O’Neachtain in his office in Government Buildings on Merrion Street on December $19^{\text {th }}, 2007$ and lasted approximately one hour and thirty minutes.

Dr Tony Colgan -Director of Advisory Services, Radiological Protection Institute Ireland (RPII), with Dr Ciara McMahon, Head of Emergency Planning, RPII, and Ms Marie Kelly, Head of Communications, RPII. The one-hour interview took place in Dr Colgan's office at the RPII headquarters in Clonskeagh, Dublin on January $10^{\text {th }}$, 2008. All three participants contributed to the interview and their comments are recorded.

Superintendent Liam King - Crime and Security Branch, and head of major emergency planning, the Garda Síochána (GS) and a member of the National Steering Group. The interview was conducted with Superintendent King in his office at Garda Headquarters in the Phoenix Park on October 17 ${ }^{\text {th }}, 2007$ and lasted approximately one hour.

Ms Sinead McSweeney - In the course of the interviews an emergent interview took place with the newly appointed Director of Communications for the Gardai, Sinead McSweeney. The interview took place in her office in Garda Headquarters in the Phoenix Park on December $12^{\text {th }}, 2007$ and lasted for 45 minutes.

Mr Padraig McKeown - Managing Director of Drury Communication, a leading PR firm in Ireland. The interview was conducted with Mr McKeown in Bewleys' Hotel, Ballsbridge, Dublin on December $20^{\text {th }}, 2007$, and lasted approximately one hour. This interview provides a useful insight into how a leading professional communication practitioner perceives the government's management of strategic major emergency communication.

Mr John Brophy - Independent crisis and risk management consultant with specific expertise in major emergency management. Mr Brophy was interviewed by way of a semi-structured interview in his office in Naas, Co. Kildare in March 2008. 
Mr John Guinane - Former military advisor at the Office of Emergency Planning. Mr Guinane was interviewed using a semi-structured interview technique. The interview took place in a restaurant in Dublin in December 2006 and lasted approximately one hour. He provided many useful insights into the workings of major emergency management in Ireland.

\section{E-mail and telephone interviews - Ireland}

In order to ascertain the likelihood of natural major emergencies occurring in Ireland, interviews were conducted by e-mail and telephone with the following experts:

Prof. Mike Williams - Geology Department, NUI Galway. A number of e-mails were exchanged in 2005 and once again in 2007. A follow-up phone-call was made in October 2007. Prof. Williams provided an insight into a big freeze that took place in Ireland in the nineteenth Century.

Dr John Tyrrell - Geography Department, University College Cork. A number of e-mails were exchanged in 2005 and once again in 2007. A follow-up phonecall was made in October 2007. Dr Tyrrell provided an insight into the likelihood of a major storm or tornado causing a major emergency situation in Ireland. 


\section{$\underline{\text { Internationally }}$}

Following a process of short-listing the three candidate countries for this research, contact was established with the government agency responsible for major emergency management in each country in turn. The agencies chosen were the Federal Emergency Management Agency (FEMA) in the US, the Swedish Emergency Management Agency (SEMA), and the Civil Contingencies Secretariat (CCS) in the UK. Each of these agencies proposed a senior communication practitioner to participate in the research.

As mentioned earlier in Chapter 1, it was decided to send a comprehensive selfadministered questionnaire to the three participants, Daryl Madden in FEMA, Malin Modh in SEMA and Carol McCall in Cabinet Office Communications (COC). The questionnaire was of considerable length ( 80 questions). The three questionnaires were dispatched by post in March 2007. Two of the completed questionnaires were received at the end of May 2007 and the third received in January 2008. Following receipt of individual questionnaires, two follow-up phone calls were made to each of the participants in order to clarify a number of their answers and also to probe further a number of issues. These phone calls were limited to twenty minutes each and the interviews recorded with the permission of the participants. The information was then transcribed and compared with the data gleaned from the questionnaires. Additionally, a number of e-mails were exchanged with the participants in order to address specific points.

The details of the three international participants are as follows:

\section{The United States}

Mr Daryl Madden - Senior public affairs manager at the Federal Emergency Management Agency (FEMA) headquarters in Washington DC. Mr Madden was chosen by FEMA's Head of External Affairs to participate in this research. The completed questionnaire was returned in June 2007. In order to receive 
clarification on a number of points, two follow-up e-mails were exchanged and one phone-call made to Mr Madden in July 2007.

\section{The United Kingdom}

Ms Carol McCall - Head of UK Security, Intelligence and Resilience

Communications/News Co-ordination Centre, at the Cabinet Office

Communications (COC) and Civil Contingencies Secretariat (CCS) in London.

The completed questionnaire was returned in February 2008. A number of phone-calls were made to Ms McCall between June 2007 and February 2008. In order to receive clarification on a number of points, one follow-up e-mail was exchanged with Ms McCall in March 2008.

\section{Sweden}

Ms Malin Modh - Principal Administrative Officer, Crisis Communication at the Swedish Emergency Management Agency (SEMA), Stockholm. The completed questionnaire was returned in May 2007. In order to receive further information and clarification on a number of points, telephone conversations took place in September 2007 and two e-mails were exchanged in August 2007. In Swedish, SEMA is known as KBM (Krisberedskapsmyndigheten). However, KBM maintains an English profile in the name SEMA and the English title has been used throughout this dissertation. 


\section{$\underline{\text { Theorists }}$}

Additionally, in order to clarify a number of theoretical points in relation to different elements of communication the following theorists were contacted by e-mail in the course of the research.

Prof. James Grunig - Professor Emeritus for the Department of Communication at the University of Maryland, and author of a number of seminal texts on the subject of strategic communication management. I engaged with Prof. Grunig on three occasions, by e-mail, during 2006 in order to clarify a number of points in relation to his model of strategic communication management.

Prof. Baruch Fischhoff - is Howard Heinz University Professor, in the Department of Social and Decision Sciences and Department of Engineering and Public Policy at Carnegie Mellon University. I was in correspondence with Prof. Fischhoff on two occasions by e-mail in June 2007 in order to clarify a number of issues on the evolution of communication management models.

Dr Peter Sandman - a former Professor at Rutgers University, Dr Sandman is a renowned practitioner of risk and crisis communication, and maintains a very useful and often cited website. I contacted Dr Sandman, by e-mail, in 2005 on a number of occasions in order to clarify a number of points related to major emergency communication theory. Through this engagement I received information concerning a number of useful websites and papers from Dr Sandman. 


\section{APPENDIX B - A RECOMMENDED OUTLINE STRUCTURE OF A MAJOR EMERGENCY RESPONSE COMMUNICATION PLAN (MERCP)}

1. Cover page: This identifies the document as a major emergency communication plan. Included on this page is the title, date issued, security classification, number of copies, and revision date.

2. Foreword: This is a letter written by a senior official, ideally the Minister with responsibility for major emergency planning and response. The purpose of this foreword is to emphasise the importance of the document.

3. Table of contents.

4. Distribution list: Details who is entitled to receive a copy of the plan.

5. Acknowledgements page: A removable page that should be signed by persons who are obliged to read and understand the MERCP.

6. Record of rehearsal dates: This should include dates and the types of rehearsal conducted.

7. Statement of overall policy: This is the overall mission statement in relation to major emergency planning, preparing, response and recovery.

8. Statement of communication policy. This is the major emergency communication mission statement.

9. Organisational structure: A wire diagram should be developed that clearly indicates the relationship of all key personnel and response organisations including, the overall major emergency commander, the major emergency team, the communication team, affiliate and partner organisations.

10. Declaration and notification procedures: This provides clear guidance for declaring a major emergency and the procedures for notifying key personnel.

11. Communication team contact sheet: This lists the names and appointments of the major emergency communication team (MECIT), including the team commander and deputies. The MECIT contact sheet should include names, addresses, particular areas of expertise for each team member, method of activating each individual, contact details (day and night), details of close relatives and neighbours, and recall procedures for each team member, such as a cascade system of notification. 
12. Designate official spokespersons: Accredited official spokespersons should be chosen for each major emergency type along with day and night contact details, and details regarding their expertise and training received.

13. Other MECITs: Contact details for communication team managers of partner/affiliate response agencies should be listed.

14. Information partners: Contact numbers for major emergency information partners including weather, public health, radiological institute, the Environmental Protection Agency, Office of Public Works, university and hospital laboratories should be listed. This can be listed separately or else as part of the stakeholder network mentioned in the next paragraph.

15. Stakeholder network: As previously described earlier, a detailed stakeholder network should be researched and developed and listed in the MERCP. This should include different groups of stakeholders, why they are listed as stakeholders, what the group represents and how they might be of use, the name of a key contact person and spokesperson for that group along with day and night contact details.

16. Third-party spokespersons: A list of third party spokespersons from that can provide support and validation to MECIT messages should be researched and listed along with contact numbers.

17. National media contact list: This list should contain information in relation to the national media including television, radio, broadsheet, tabloid, trade, specialised print and web-based media. Information to be listed includes a profile of their average viewer/listener/reader, names of key journalists and editors, mobile phone numbers, after hours news desk contact numbers, e-mail addresses, fax numbers. A record of when these numbers and e-mails were last tested should also be included.

18. Local/regional media contact list: A list of local media is extremely important and due to the number and diversity of outlets, this contact list will be more difficult to prepare. The same details provided in para. 17 will be required. As most local media will not have a 24-hour news desk, a list of mobile numbers will be important.

19. MECIT responsibilities: Clear roles and responsibilities should be designated to each member of the MECIT. This avoids important roles being overlooked or duplication of roles. Each individual role should be analysed, the important tasks listed and members of the MECIT assigned to cover each task. The roles to be considered include the communication team leader, on-site liaison, media liaison, public affairs liaison, stakeholder/partner liaison, victim and family liaison, information 
management, researchers, copywriters, CIMC manager, media centre manager, and record management.

20. Coordination with affiliate MECITs. Clear procedures need to be put in place to allow coordination with each of the affiliate/partner MECITs listed in para. 13 above. This should include coordination of command and control, release of information, and processes for remaining on message.

21. Major emergency strategy matrix: This matrix is a very comprehensive tool that plots the specific major emergencies that have been identified, considered likely, and placed in order of priority. The matrix should also specify particular problems that exist in relation to each identified major emergency type and describe the communication problems associated with each of these problems. The matrix will also detail major emergency communication goals and objectives for each of the identified communication problems, along with details of target audiences and key messages to be disseminated to overcome each individual problem.

22. Information security: Clear guidelines regarding the security of sensitive information and proprietary information. This includes information in relation to state security or intelligence, trade secrets or corporate confidentiality, and also the names of major emergency victims before families have been informed.

23. Internal information clearance/approval procedures: This should state who is authorised to release information to major emergency teams and other affiliate response organisations. Information review, verification and release procedures should be clearly stated with no room for ambiguity.

24. External information clearance: This should include who is authorised to release information to external stakeholders and publics, including the media. Information review, verification and release procedures should be clearly stated with no room for ambiguity.

25. Cooperation with partners: The MERCP should clearly state the procedures for operating with all affiliate and partner MECITs operating within the communication and information management centre (CIMC).

26. Procedures to scale-up the communication function: These should be stated in case the situation dictates that there is a need to scale-up the entire response effort.

27. Channels of communication: These should include a web-site, an internet notice board, and a 1-800 low-call phone system. The MECIT must also provide a mechanism for producing large amounts of quality booklets, leaflets, posters, bill-boards, mail-shots, graphics, and video clips. 
Details of agreements and subsequent procedures should be stated in the MERCP for employing television and radio stations for carrying of warning and alarm messages, along with instructional information. Any similar agreements with mobile telecommunication operators to send important warning or instructional text messages to publics at risk should also be stated.

28. Guidance templates: A number of templates or checklists should be included in the MERCP that will prove to be very useful in guiding the major emergency communication team. The following need to be included:

a. A tick-box checklist of all steps to be taken during the first number of hours of a major emergency.

b. A list of questions that the press are likely to ask which MECIT press officers can begin to individualise once a major emergency occurs.

c. Do's and don'ts list for dealing with the media.

d. Media interview guide - tips for conducting different types of media interviews.

e. A worksheet to guide media content analysis.

f. Generic press releases.

g. Guidelines for notifying next-of-kin regarding news of death or serious injury.

h. Sample letter of sympathy.

i. Sample obituary.

j. Layout of the CIMC and media centre and a list of equipment and supplies required as discussed earlier in Chapter 5.

k. Public information call-tracking $\log$ and information-request log.

1. Communication action log to record all communications initiated.

m. Press conference checklist. 
APPENDIX C - A COPY OF THE SELF-ADMINISTERED QUESTIONNAIRE COMPLETED BY A MAJOR EMERGENCY COMMUNICATION OFFICIAL IN THE SWEDISH EMERGENCY MANAGEMENT AGENCY (SEMA).

CORRESPONDING QUESTIONNAIRES WERE COMPLETED BY OFFICIALS IN THE UNITED KINGDOM (CCS) AND THE UNITED STATES (FEMA). 


\section{Major Emergency Communication}

Questionnaire for United Kingdom Civil Contingencies Secretariat

From:- Paul Connors - Doctoral Student - Dublin City University

Supervisor:- Professor Colum Kenny

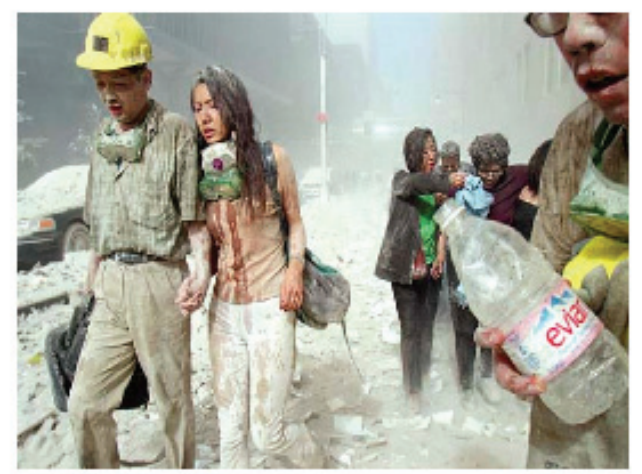

A recommended framework for the Irish Government for the strategic management of communication throughout all stages of a major emergency lifecycle 


\begin{abstract}
APPENDIX D - A COPY OF THE SEMI-STRUCTURED QUESTIONNAIRE USED AS A GUIDE DURING THE INTERVIEW WITH A MAJOR EMERGENCY COMMUNICATION OFFICIAL FROM THE IRISH HEALTH SERVICES EXECUTIVE (HSE) .

CORRESPONDING SEMI-STRUCTURED QUESTIONNAIRES WERE USED DURING INTERVIEWS WITH OTHER OFFICIALS FROM IRISH GOVERNMENT DEPARTMENTS AND AGENCIES WITH RESPONSIBILITY FOR MAJOR EMERGENCY MANAGEMENT.
\end{abstract}




\section{Interview with Gavin Maguire Health Service Executive}

\section{State your full name \& title and appointment in relation to major emergency planning}

Who within the HSE is considered the senior major emergency communication practitioner - decides the model adopted, strategy, policy and protocols?

\section{Major Emergency Management Framework}

Do you think the framework is strategically focused?

Should the framework have a statutory basis?

Is the DoD the best placed Department to have oversight?

Is there any other Dept better placed to have oversight?

What is your opinion on the oversight role of the OEP - comment on its ability to oblige response agencies to plan, train, conduct exercises, and report on the achievement or not of their goals.

Some commentators have described the planning effort as a 'plethora of committes and working groups working parallel - and often in isolation - Do you agree?

\section{Communication Strategy within the Framework}

In your opinion -who is responsible for overseeing the strategic communication management role?

If I told you a person within the HSE was to become a Communication Manager- what functions would you suggest this person should be responsible for?

(prompt) What about any of the following:

public information -

media management - 
information management -

dealing with survivors -

relatives \& friends -

vulnerable persons -

and internal communication.

Is it your opinion that the new framework offers a joined-up approach to all of these functions?

Within the US JIC model these roles are placed under one appointment "information officer" - do you see merit in this? Could it apply to Ireland?

The new framework document places significant attention on communication management following the actualisation of a major emergency:

Do you think that sufficient attention has been given to the communication function in the pre-crisis stage - and in the post-crisis stage of a major emergency?

\section{Communication Strategy}

I see that the HSE in its National Pandemic Influenza Plan considers communication strategy to be the top of its list of eight core elements of a plan - why?

How strategic is the HSE's approach to ME communication?

What in your opinion are the key elements of a communication strategy - ie what makes it strategic?

Is a strategic approach important - why?

Do you think that the influenza plan provides sufficient long-term focus?

(probe school system etc)

Do you consider the communication element of the new framework strategic?

Why? 
Throughout many countries - considered leaders in major emergency managment - a similar model of major emergency communication seems to be emerging. This includes-engaging in communication research and evaluation - testing messages - building up trust and credibility - and greater dialogue with stakeholders and publics, communicating with rather than merely communicating to those affected by major emergencies. Do you think this model is likely to work within the Irish cultural context?

\section{Communication Planning}

Who is responsible for communication planning in the the HSE?

Is there a communication plan in place?

Importance of flexibility in the communication plan? 1-10

Have you heard of scenario planning? Does the HSE use it?

\section{Broad-based deliberation}

Participating in broad-based deliberation means engaging in a meaningful way with interested and affected stakeholders and publics in relation to decisions that are likely to affect them in some way throughout all stages of a major emergency.

Do you support this process? For both stakeholders and publics Identify advantages to pursuing this process in relation to planning and response?

Identify disadvantages?

On a scale of 1-10 how important is this process? Stakeholders / Public

On a scale of $1-10$ can you rate how much the planning process would be disrupted? Stakeholders / Public

\section{Research}

Does the HSE engage in communication research into attitudes, concerns, opinions, needs of? 
Someone once described producing a communication strategy without communication based research as being inherently flawed do you agree?

On a scale of 1-10 how important is this process?

\section{Openness \& Transparency}

How important is openness and transparence with the public in relation to major emergency management?

Currently - how open and transparent is the Irish Gov in relation to MEM 1-10?

Should the public have full access to all of the details in relation to information that may affect them?

Is full transparency likely to cause worry or worse panic?

Are politicians and civil servants afraid of another Joe Jacobs affair?

\section{Trust \& Credibility}

What to you are the key elements of instilling trust \& credibility amongst the public and media?

How can trust \& credibility be achieved?

Would engaging with the public in a broad-based deliberative approach help in this regard?

\section{Key messages}

Please describe the process of developing key messages within the HSE

Do you ever test these messages? How?

Did you test any of the messages for the influenza plan - do you think that the information in the document is accessible to the general public? 


\section{Channels of communication}

Did the HSE conduct research into optimum channels of communication for its proposed communication strategy for the Influenza document?

(prompt - leaflet drop - press briefings - ad campaign - telephone hotline $-\mathrm{tv} \&$ radio updates)

What about marginalised communities - travellers - disabled?

Are these tested? When?

The strategic framework makes mention of certain channels of communication - leaflet drop - hotline - media etc In your opinion - is this sufficient to reach all types of publics?

\section{Evaluation of communication strategy}

Does the HSE ever evaluate the efficacy of messages disseminated?

Does this evaluation assess the message dissemination process or the outcome - or both?

If this is done - who carries it out - and briefly describe the process?

\section{Knowledge of communication practitioners}

How do you choose your communication practitioners?

Do you provide professional communication training for them?

Have any of them got professional education? Communications degrees - masters - $\mathrm{PhD}$

Rate from 1-10 the importance that the HSE attaches to its communication practitioners having a communications related academic qualification?

Training \& Exercising How important is training \& exercising the communication function to the HSE?

Does somebody undertake specific communication input to exercises? - if so who assesses this? 
